\title{
Copper-Catalyzed Synthesis of Indolyl Benzo[b]carbazoles and Their Photoluminescent Property
}

\author{
Tonggang Hao ${ }^{a}$, Long Huang ${ }^{a}$, Yin Wei ${ }^{a}$, and Min Shi ${ }^{*}$ \\ ${ }^{a}$ State Key Laboratory of Organometallic Chemistry, Center for Excellence in Molecular Synthesis, \\ University of Chinese Academy of Sciences, Shanghai Institute of Organic Chemistry, Chinese \\ Academy of Sciences, 345 Lingling Road, Shanghai 200032 P. R. China. E-mail: \\ mshi@mail.sioc.ac.cn
}

\section{CONTENTS}

1. General Remarks. .2

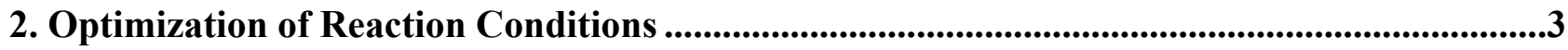

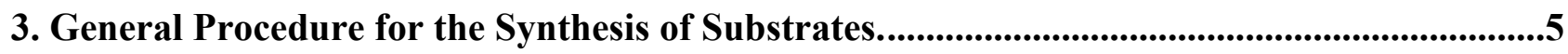

4. General Procedure for the Synthesis of Products. ................................................................9

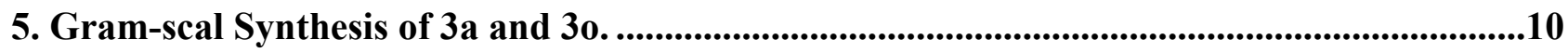

6. UV-Vis Absorption Spectroscopy, Fluorescence Emission Spectroscopy and Fluorescence Quantum Yields .....................................................................................................................................11

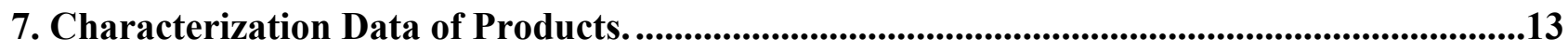

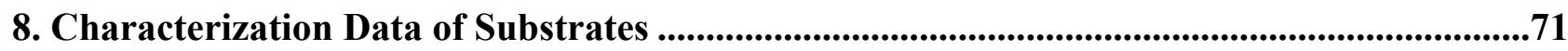

9. X-ray Data........................................................................................................................................121

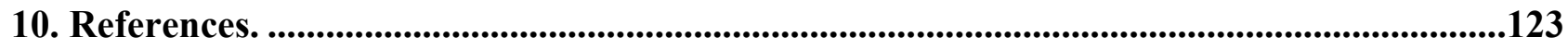




\section{General Remarks.}

${ }^{1} \mathrm{H}$ NMR spectra were recorded on a Varian Mercury-400 spectrometer for solution in $\mathrm{CDCl}_{3}$ with tetramethylsilane (TMS) as an internal standard or in $\mathrm{CD}_{2} \mathrm{Cl}_{2}$; coupling constants $J$ are given in Hz. ${ }^{13} \mathrm{C}$ NMR spectra were recorded on a Varian Mercury-400 and 600 spectrophotometers (100 $\mathrm{MHz})$ with complete proton decoupling spectrophotometers $\left(\mathrm{CDCl}_{3}: 77.0 \mathrm{ppm}, \mathrm{CD}_{2} \mathrm{Cl}_{2}: 33.4 \mathrm{ppm}\right)$. Mass and HRMS spectra were recorded by EI, ESI or FI method. Organic solvents used were dried by standard methods when necessary. Infrared spectra were recorded on a Perkin-Elmer PE-983 spectrometer with absorption in $\mathrm{cm}^{-1}$. Melting points were determined on a digital melting point apparatus and temperatures were uncorrected. The reactions were carried out in oil bath. Commercially obtained reagents were used without further purification. All these reactions were monitored by TLC with silica gel coated plates. Flash column chromatography was carried out using silica gel at increased pressure. Substrates $\mathbf{2 a}, \mathbf{2 m}, \mathbf{2 0}, \mathbf{2 p}$ are commercially available from Bidepharm. It should be mentioned here that some of our isolated products contain a small amount of impurity due to their poor solubility in organic solvents. They are not soluble in $\mathrm{MeCN}, \mathrm{MeOH}$, EtOH, toluene or $\mathrm{CHCl}_{3}$ and only partially soluble in $\mathrm{CH}_{2} \mathrm{Cl}_{2}$. Using $\mathrm{CH}_{2} \mathrm{Cl}_{2}$ and petroleum ether as eluent caused a serious tailing in silica gel chromatography purification. In addition, substrates 1 were unstable in neat, thus some of NMR spectra were not clean. 


\section{Optimization of Reaction Conditions}

Table S1: Optimization of the catalysts for the production of $\mathbf{3 a}$.

\begin{tabular}{|c|c|c|c|c|}
\hline \multirow{2}{*}{ entry ${ }^{a}$} & \multirow{2}{*}{ catalyst } & \multirow{2}{*}{ solvent } & \multicolumn{2}{|c|}{ yield $/ \%^{b}$} \\
\hline & & & $3 a$ & $3 b$ \\
\hline 1 & $\mathrm{Cu}(\mathrm{OTf})_{2}$ & DCM & $98(94)^{c}$ & 0 \\
\hline 2 & $\mathrm{BF}_{3} \cdot \mathrm{OEt}_{2}$ & DCM & 89 & 0 \\
\hline 3 & $\mathrm{Yb}(\mathrm{OTf})_{3}$ & DCM & 33 & 0 \\
\hline 4 & $\operatorname{Tm}(\mathrm{OTf})_{3}$ & DCM & 56 & 0 \\
\hline 5 & $\mathrm{Ga}(\mathrm{OTf})_{3}$ & DCM & 51 & 0 \\
\hline 6 & $\ln (\mathrm{OTf})_{3}$ & $\mathrm{DCM}$ & 88 & 0 \\
\hline 7 & $\mathrm{Y}(\mathrm{OTf})_{3}$ & DCM & 18 & 0 \\
\hline 8 & $\mathrm{Sc}(\mathrm{OTf})_{3}$ & DCM & 90 & 0 \\
\hline 9 & $\mathrm{CuSO}_{4}$ & DCM & 0 & 0 \\
\hline 10 & HOTf & DCM & 82 & 0 \\
\hline 11 & $\mathrm{Cu}(\mathrm{OTf})_{2}{ }^{d}$ & DCM & 51 & 0 \\
\hline 12 & $\mathrm{Cu}(\mathrm{OTf})_{2}^{\mathrm{e}}$ & $\mathrm{DCM}$ & 92 & 0 \\
\hline 13 & $\mathrm{CeCl}_{3}$ & DCM & 0 & 40 \\
\hline 14 & $\mathrm{AgSbF}_{6}$ & DCM & 0 & 52 \\
\hline 15 & - & DCM & 0 & 0 \\
\hline
\end{tabular}

a Reaction conditions: 1a (3 mmol), 2a (4 mmol), LA (10 mol\%), solvent (2.0 mL), under an Ar atmosphere for $3 \mathrm{~h}$, in a sealed tube. ${ }^{\mathrm{b}} \mathrm{NMR}$ yield using $\mathrm{CH}_{2} \mathrm{Br}_{2}$ as an internal standard. ${ }^{\mathrm{c}}$ Isolated yield. ${ }^{\mathrm{d}}$ Use $5 \mathrm{~mol} \% \mathrm{Cu}(\mathrm{OTf})_{2}$ as the catalyst. ${ }^{\text {e }}$ Use $20 \mathrm{~mol} \% \mathrm{Cu}(\mathrm{OTf})_{2}$ as the catalyst. 
Table S2: Optimization of the solvents for the production of $\mathbf{3 a}$.

\begin{tabular}{cccc} 
& & \\
entry $^{a}$ & catalyst & solvent & yield $/ \%^{b}$ \\
\hline 1 & $\mathrm{Cu}(\mathrm{OTf})_{2}$ & THF & 26 \\
2 & $\mathrm{Cu}(\mathrm{OTf})_{2}$ & DMSO & 0 \\
3 & $\mathrm{Cu}(\mathrm{OTf})_{2}$ & Toluene & 54 \\
4 & $\mathrm{Cu}(\mathrm{OTf})_{2}$ & DCM (wet) & 62 \\
$\mathrm{Cu}(\mathrm{OTf})_{2}$ & &
\end{tabular}

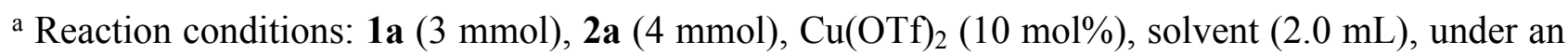
Ar atmosphere for $3 \mathrm{~h}$ in a sealed tube. ${ }^{\mathrm{b}} \mathrm{NMR}$ yield using $\mathrm{CH}_{2} \mathrm{Br}_{2}$ as an internal standard. 


\section{General Procedure for the Synthesis of Substrates.}

\section{General procedure for the synthesis of compounds 1a-f.}
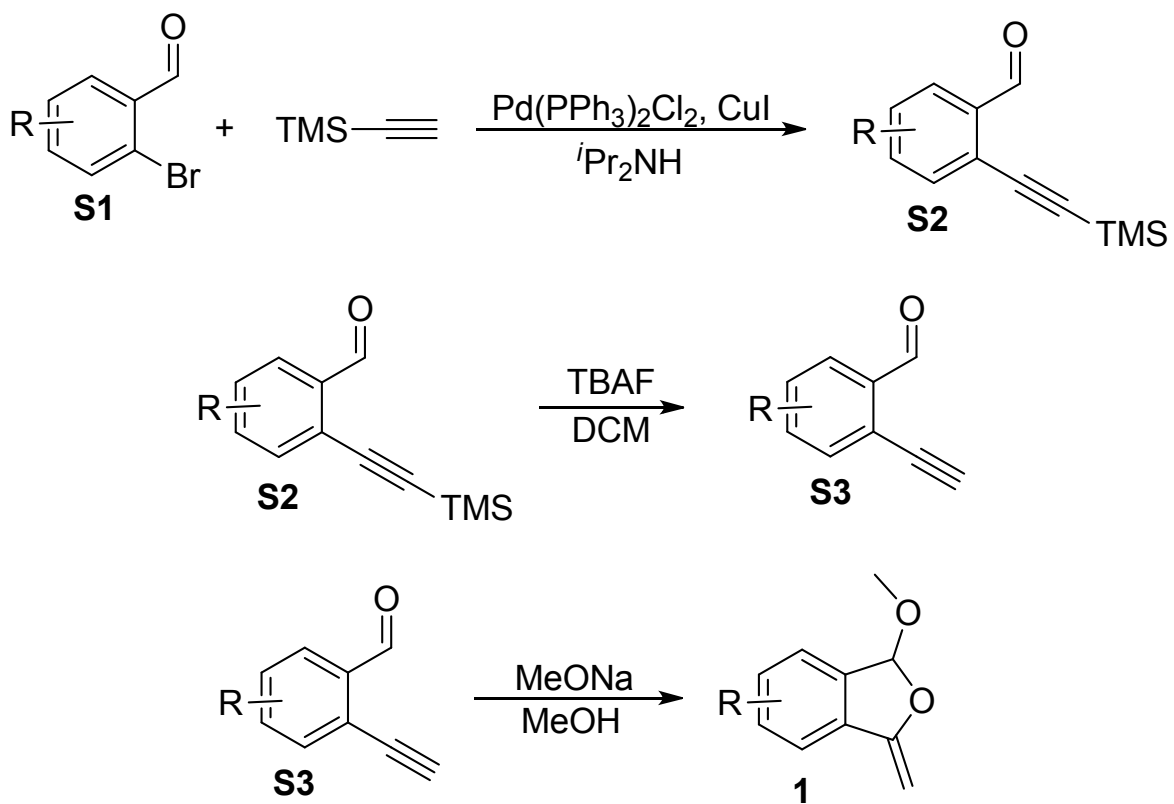

The procedure of preparing substrates 1 was slightly modified according to the previous literature. ${ }^{1}$

To a stirred solution of $\mathrm{PdCl}_{2}\left(\mathrm{PPh}_{3}\right)_{2}(2.0 \mathrm{~mol} \%)$ and $\mathrm{CuI}(4.0 \mathrm{~mol} \%)$ in $\mathrm{i}-\mathrm{Pr}_{2} \mathrm{NH}(30.0 \mathrm{~mL})$ was added S1 (1.0 equiv) and trimethylsilylacetylene (1.2 equiv) under argon atmosphere. The resulted mixture was stirred at $80{ }^{\circ} \mathrm{C}$ for $8 \mathrm{~h}$. After the filtration and the removal of solvent under reduced pressure, the residue was purified by a column chromatography on silica gel (eluent: petroleum ether $/$ ethyl acetate $=20 / 1$ ) to afford the corresponding compounds $\mathbf{S} 2$ in good yields ranging from $55 \%$ to $86 \%$.

To a solution of $\mathbf{S 2}$ (1.0 equiv) in $10.0 \mathrm{~mL}$ DCM was added TBAF (1.2 equiv, $1 \mathrm{M}$ in THF) at room temperature, and the reaction mixture was stirred for 1 hour. After the reaction completion monitored by TLC analysis, the residue was purified by a silica gel flash chromatography (eluent: petroleum ether / ethyl acetate $=20 / 1$ ) to afford compounds $\mathbf{S 3}$ in good yields ranging from $80 \%$ to $90 \%$.

To a solution of $\mathbf{S 3}$ (1.0 equiv) in $5 \mathrm{~mL} \mathrm{MeOH}$ was added $\mathrm{MeONa}$ ( 0.5 equiv) under argon atmosphere, and the reaction mixture was stirred for 1 hour at $75{ }^{\circ} \mathrm{C}$. After the reaction completion monitored by TLC analysis, the residue was purified by an aluminum oxide flash chromatography (eluent: petroleum ether / ethyl acetate $=10 / 1$ ) to afford compounds $\mathbf{1}$. The compounds $\mathbf{1}$ will easily undergo condensation when it is in a neat state, and they should be carefully concentrated 
upon removing the solvent till $0.5 \mathrm{~mL}$ and then were directly used in the next operation. 

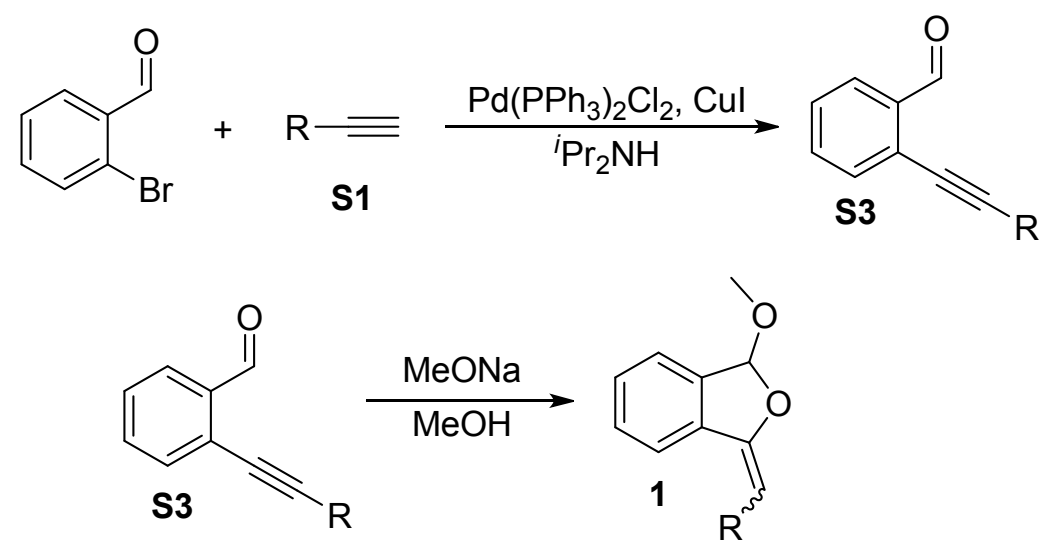

The procedure of preparing substrates 1 was slightly modified according to the previous literature. ${ }^{1}$

To a stirred solution of $\mathrm{PdCl}_{2}\left(\mathrm{PPh}_{3}\right)_{2}(2.0 \mathrm{~mol} \%)$ and $\mathrm{CuI}(4.0 \mathrm{~mol} \%)$ in i- $\mathrm{Pr}_{2} \mathrm{NH}(30.0 \mathrm{~mL})$ was added 2-bromobenzaldehyde (1.0 equiv) and $\mathbf{S 1}$ (1.0 equiv) under argon atmosphere and the resulted mixture was stirred at $80{ }^{\circ} \mathrm{C}$ for $8 \mathrm{~h}$. After the filtration and the removal of solvent under reduced pressure, the residue was purified by a column chromatography on silica gel (eluent: petroleum ether $/$ ethyl acetate $=20 / 1$ ) to afford the corresponding compounds $\mathbf{S} 2$ in good yields ranging from $60 \%$ to $84 \%$.

To a solution of $\mathbf{S 2}$ (1.0 equiv) in $5 \mathrm{~mL} \mathrm{MeOH}$ was added $\mathrm{MeONa}(0.5$ equiv) under argon atmosphere, and the reaction mixture was stirred for 1 hour at $75{ }^{\circ} \mathrm{C}$. After the reaction completion monitored by TLC analysis, the residue was purified by an aluminum oxide flash chromatography (eluent: petroleum ether / ethyl acetate $=10 / 1$ ) to afford compounds $\mathbf{1}$. The compounds 1 will easily undergo condensation when it is in a neat state, and therefore, they should be carefully concentrated upon removing the solvent till $0.5 \mathrm{~mL}$ and then were directly used in the next operation. 
General procedure for the synthesis of compounds $2 a-k$, and $2 p$.

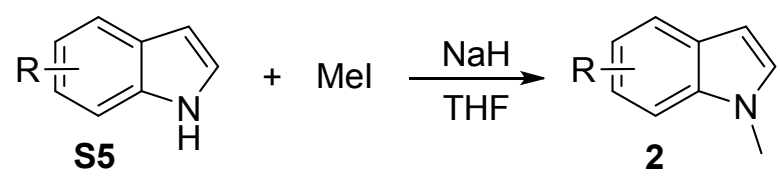

The procedure of preparing substrates 2 was slightly modified according to the previous literature. ${ }^{2}$

To a stirred solution of $\mathbf{S 5}$ (1.0 eq) in THF placed in an ice buffer was added $\mathrm{NaH}$ (1.2 eq) slowly. Then the indomethane (1.1 eq) was added dropwise. After 4 hours, methanol $(5 \mathrm{~mL})$ was added and after the removal of solvent under reduced pressure, the residue was purified by a column chromatography on silica gel (eluent: petroleum ether / ethyl acetate $=20 / 1$ ) to afford the corresponding compounds 2 in good yields ranging from $85 \%$ to $98 \%$. 
General procedure for the synthesis of compounds 2l-n.

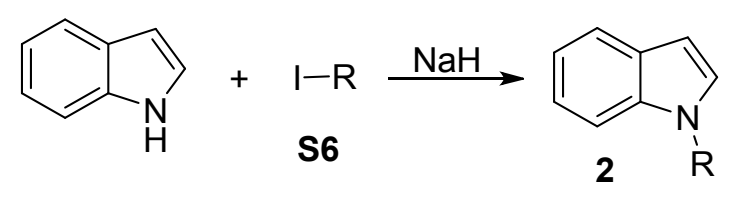

The procedure of preparing substrates $\mathbf{2}$ was slightly modified according to the previous literature. ${ }^{2}$

To a stirred solution of indole (1.0 eq) in THF placed in an ice buffer was added NaH (1.2 eq) slowly. Then S6 (1.1 eq) was added dropwise. After 4 hours, methanol (5 mL) was added and after the removal of solvent under reduced pressure, the residue was purified by a column chromatography on silica gel (eluent: petroleum ether $/$ ethyl acetate $=20 / 1$ ) to afford the corresponding compounds 2 in good yields ranging from $65 \%$ to $87 \%$. 
4. General Procedure for the Synthesis of Products.
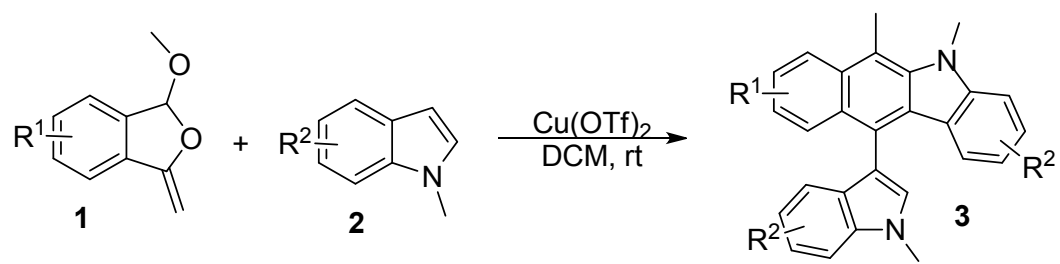

To a stirred solution of $\mathbf{1}(0.3 \mathrm{mmol})$ and $\mathbf{2}(0.4 \mathrm{mmol})$ in $2 \mathrm{~mL}$ anhydrous dichloromethane, $\mathrm{Cu}(\mathrm{OTf})_{2}(0.1 \mathrm{eq})$ was added under argon atmosphere and the resulted mixture was stirred at room temperature for $3 \mathrm{~h}$. After the filtration and the removal of solvent under reduced pressure, the residue was purified by a column chromatography on silica gel (eluent: petroleum ether / dichloromethane $=3 / 1$ ) to afford the corresponding compounds 3 in moderate to good yields ranging from $16 \%$ to $96 \%$. 


\section{Gram-scale Synthesis of 3a and 30.}

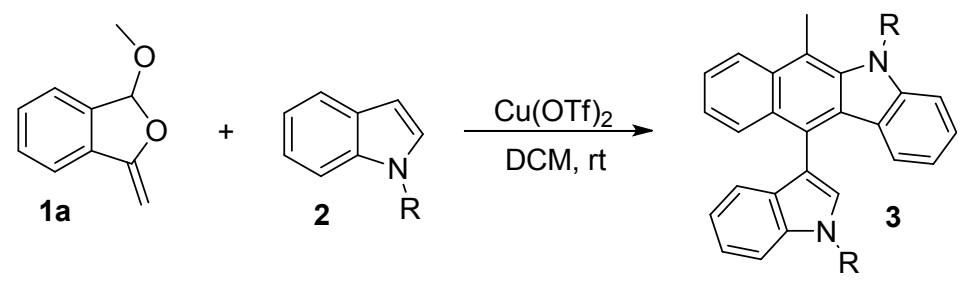

To a stirred solution of $\mathbf{1 a}(1.216 \mathrm{~g}, 7.5 \mathrm{mmol})$ and $\mathbf{2 a}(1.313 \mathrm{~g}, 10.0 \mathrm{mmol})$ in $15 \mathrm{~mL}$ anhydrous dichloromethane, $\mathrm{Cu}(\mathrm{OTf})_{2}(0.1 \mathrm{eq})$ was added under argon atmosphere and the resulted mixture was stirred at room temperature for $6 \mathrm{~h}$. After the filtration and the removal of solvent under reduced pressure, the residue was purified by a column chromatography on silica gel (eluent: petroleum ether / dichloromethane $=3 / 1$ ) to afford the crude compound 3a, and the crude product 3a was then recrystallized by solvent evaporation method (petroleum ether / dichloromethane $=6$ / 1) to afford the pure product 3a (1.121 g, 60\% yield).

To a stirred solution of $1 \mathrm{a}(0.811 \mathrm{~g}, 5.0 \mathrm{mmol})$ and $2 \mathrm{o}(1.757 \mathrm{~g}, 15 \mathrm{mmol})$ in $15 \mathrm{~mL}$ anhydrous dichloromethane, $\mathrm{Cu}(\mathrm{OTf})_{2}(0.1 \mathrm{eq})$ was added under argon atmosphere and the resulted mixture was stirred at room temperature for $6 \mathrm{~h}$. After the filtration and the removal of solvent under reduced pressure, the residue was purified by a column chromatography on silica gel (eluent: petroleum ether / dichloromethane $=1 / 1$ ) to afford the crude compound 3o, and the crude product 3o was then recrystallized by solvent evaporation method (petroleum ether / dichloromethane $=3$ / 1) to afford pure product 30 (1.034 g, 59\% yield). 
6. UV-Vis Absorption Spectroscopy, Fluorescence Emission Spectroscopy and Fluorescence Quantum Yields

Sample preparation: To a $100 \mathrm{~mL}$ of volumetric flask was added $\mathbf{3 a}$ or other products $(0.03 \mathrm{mmol})$ and diluted with $\mathrm{CH}_{2} \mathrm{Cl}_{2}$ to $25 \mathrm{~mL}$. The flask with solution was shaken several times for using. All of these products were excited at $355 \mathrm{~nm}$.

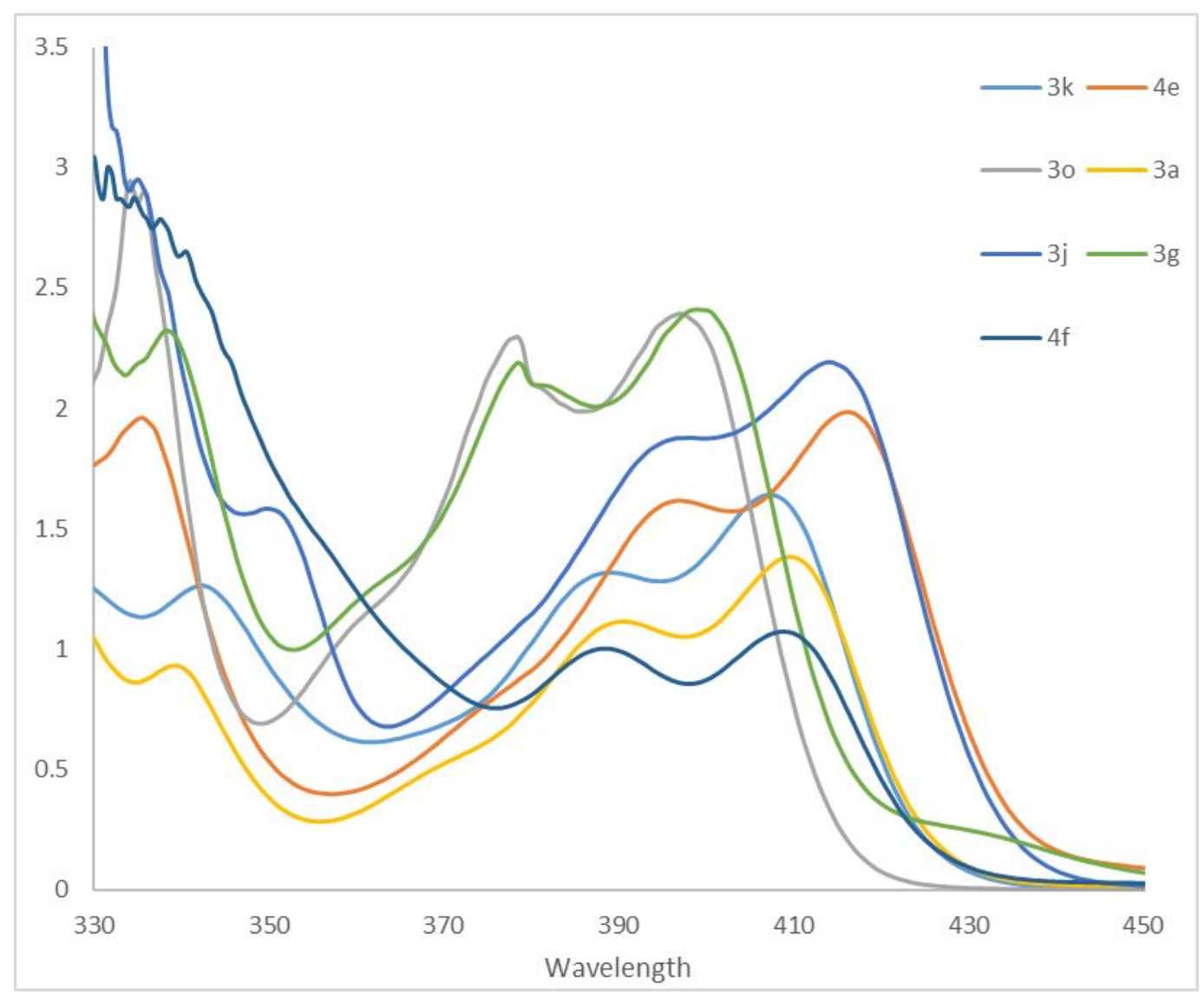

Figure S1. UV-Vis absorption spectra of $\mathbf{3 k}, \mathbf{4 e}, \mathbf{3 0}, \mathbf{3 a}, \mathbf{3 j}, \mathbf{3 g}$, and $\mathbf{4 f}$. 


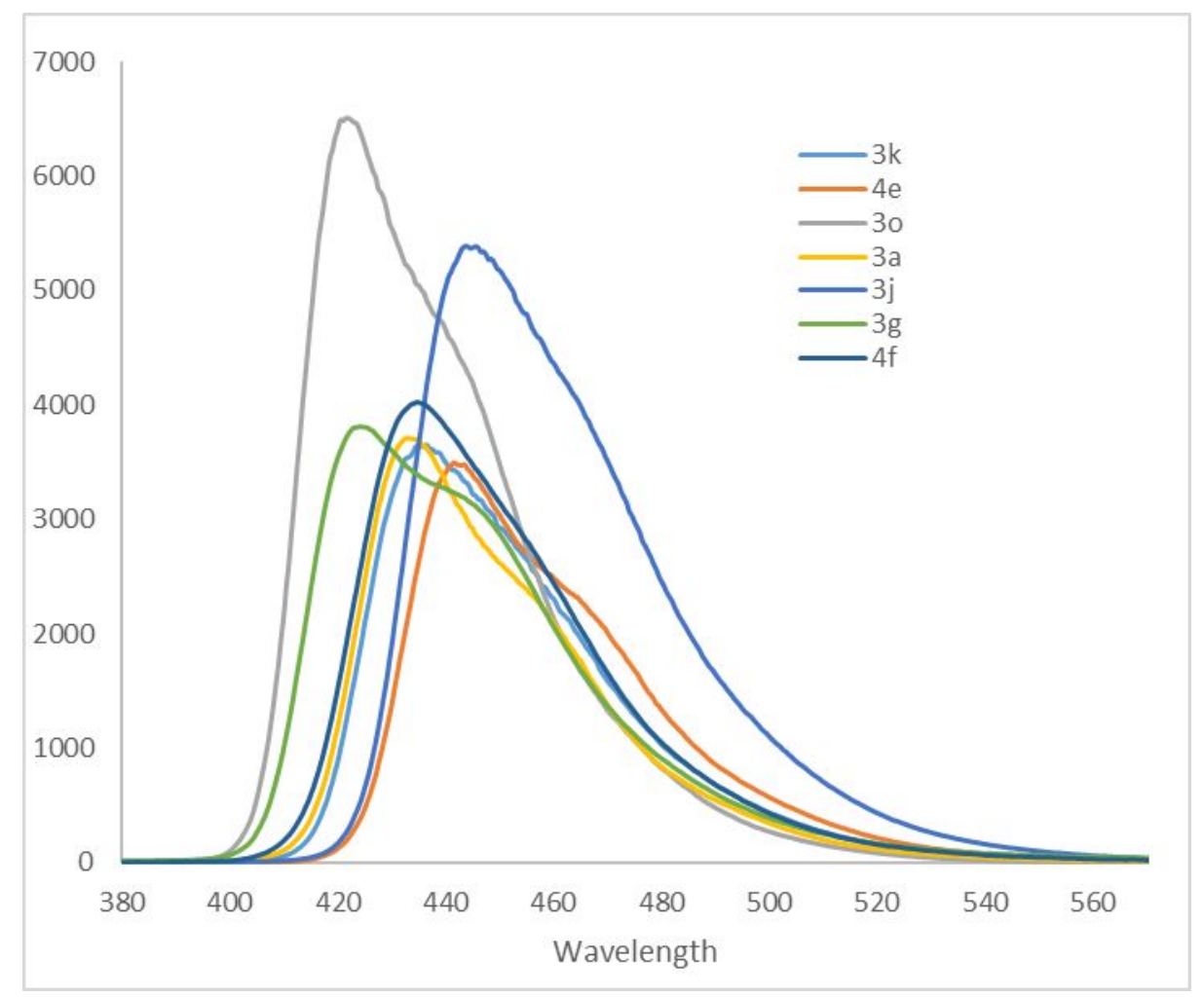

Figure S2. Fluorescence emission spectra of $\mathbf{3 k}, \mathbf{4 e}, \mathbf{3 o}, \mathbf{3 a}, \mathbf{3 j}, \mathbf{3 g}$, and $\mathbf{4 f}$.

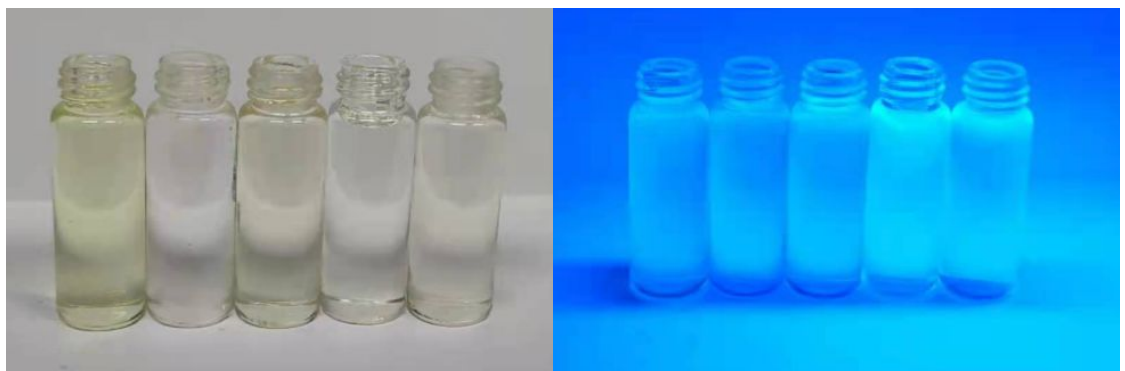

Figure S3. Fluorescence emission, without 365 UV light (lift), with 365nm UV light (right) (from left to right: $\mathbf{3 o}, \mathbf{3 j}, \mathbf{4 e}, \mathbf{3 a}$ and $\mathbf{3 k}$ ).

Quantum yield determination: All the quantum yields of samples were determined based on $1.0 \mathrm{x}$ 10-5 mol/L Quinine in 0.5 $\mathrm{M} \mathrm{H}_{2} \mathrm{SO}_{4}(\Phi=0.55)$. Fluorescence emission of all the samples were measured in $\mathrm{CH}_{2} \mathrm{Cl}_{2}, \mathrm{c}=0.3 \mu \mathrm{M}$, excited at $355 \mathrm{~nm}$ with $2.5 \mathrm{~nm}$ EX slit and $10.0 \mathrm{EM}$ slit. 


\section{Characterization Data of Products.}

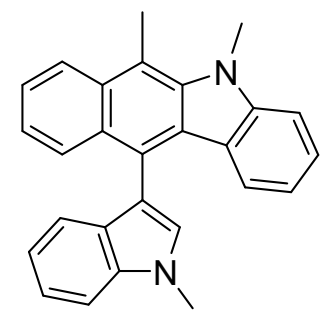

Compound 3a: Yield: $70 \mathrm{mg}$, 94\%; a yellow solid; Mp: $164-167{ }^{\circ} \mathrm{C}$; Eluent: $\mathrm{PE} / \mathrm{DCM}=3 / 1 .{ }^{1} \mathrm{H}$ NMR (400 MHz, $\left.\mathrm{CDCl}_{3}, \mathrm{TMS}\right) \delta 8.19(\mathrm{~d}, J=8.7 \mathrm{~Hz}, 1 \mathrm{H}), 7.91(\mathrm{~d}, J=8.5 \mathrm{~Hz}, 1 \mathrm{H}), 7.48(\mathrm{~d}, J=8.3$ $\mathrm{Hz}, 2 \mathrm{H}), 7.39-7.18(\mathrm{~m}, 4 \mathrm{H}), 7.13(\mathrm{~d}, J=4.7 \mathrm{~Hz}, 2 \mathrm{H}), 6.97$ (t, $J=7.5 \mathrm{~Hz}, 1 \mathrm{H}), 6.92(\mathrm{~d}, J=7.8 \mathrm{~Hz}$, 1H), $6.76(\mathrm{t}, J=7.5 \mathrm{~Hz}, 1 \mathrm{H}), 4.05(\mathrm{~s}, 3 \mathrm{H}), 3.91(\mathrm{~s}, 3 \mathrm{H}), 3.13(\mathrm{~s}, 3 \mathrm{H}) ;{ }^{13} \mathrm{C}\left\{{ }^{1} \mathrm{H}\right\}-\mathrm{NMR}(100 \mathrm{MHz}$, $\left.\mathrm{CDCl}_{3}, \mathrm{TMS}\right) \delta 145.5,140.0,137.0,132.1,129.4,128.5,128.3,127.2,126.6,125.5,124.7,124.3$, 123.4, 123.1, 122.7, 122.1, 121.8, 120.5, 119.5, 118.6, 112.3, 111.7, 109.3, 108.0, 34.4, 33.0, 14.7; IR (neat): v 3068, 2924, 1594, 1471, 1238, 1116, 1021, $736 \mathrm{~cm}^{-1}$; HRMS (FI-TOF) m/z: [M] Calcd for $\mathrm{C}_{27} \mathrm{H}_{22} \mathrm{~N}_{2}$ 374.1778; found 374.1779. 


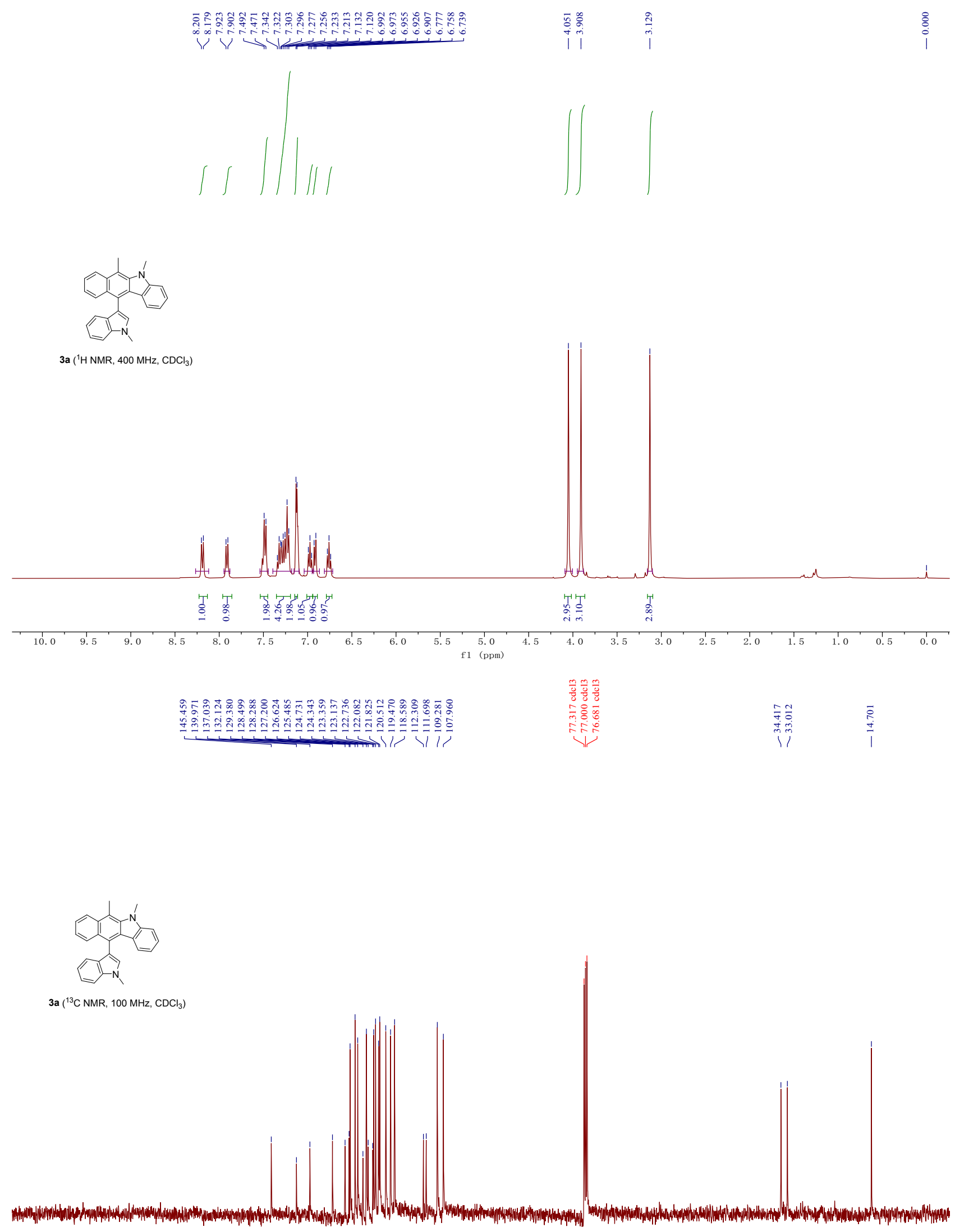

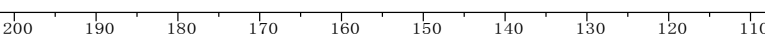




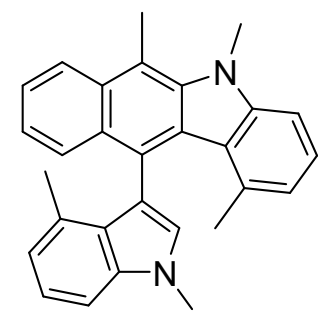

Compound 3b: Yield: $67 \mathrm{mg}, 83 \%$; a yellow solid; Mp: $>200{ }^{\circ} \mathrm{C}$; Eluent: $\mathrm{PE} / \mathrm{DCM}=3 / 1 .{ }^{1} \mathrm{H}$ NMR (400 MHz, $\mathrm{CDCl}_{3}$, TMS) $\delta 8.16(\mathrm{~d}, J=8.7 \mathrm{~Hz}, 1 \mathrm{H}), 7.75(\mathrm{~d}, J=8.6 \mathrm{~Hz}, 1 \mathrm{H}), 7.48(\mathrm{t}, J=7.6$ $\mathrm{Hz}, 1 \mathrm{H}), 7.38-7.28(\mathrm{~m}, 2 \mathrm{H}), 7.25-7.15(\mathrm{~m}, 3 \mathrm{H}), 6.93(\mathrm{~s}, 1 \mathrm{H}), 6.86(\mathrm{~d}, J=7.0 \mathrm{~Hz}, 1 \mathrm{H}), 6.75(\mathrm{~d}, J$ $=7.0 \mathrm{~Hz}, 1 \mathrm{H}), 4.11(\mathrm{~s}, 3 \mathrm{H}), 3.85(\mathrm{~s}, 3 \mathrm{H}), 3.17(\mathrm{~s}, 3 \mathrm{H}), 1.92(\mathrm{~s}, 3 \mathrm{H}), 1.54(\mathrm{~s}, 3 \mathrm{H}) ;{ }^{13} \mathrm{C}\left\{{ }^{1} \mathrm{H}\right\}-\mathrm{NMR}$ (100 MHz, $\left.\mathrm{CDCl}_{3}, \mathrm{TMS}\right) \delta_{\mathrm{C}} 147.1,141.0,136.7,135.3,131.9,131.6,130.7,130.0,129.5,128.3$, $127.7,126.9,126.8,124.9,122.8,122.7,122.3,122.1,122.0,120.8,116.2,111.1,107.1,106.1$, 35.5, 33.0, 23.1, 18.9, 15.6; IR (neat): $v$ 2914, 1574, 1464, 1243, 1071, 775, $753 \mathrm{~cm}^{-1}$; HRMS (ESI-TOF) m/z: [M] $]^{+}$Calcd for $\mathrm{C}_{29} \mathrm{H}_{26} \mathrm{~N}_{2}$ 402.2091; found 402.2096. 

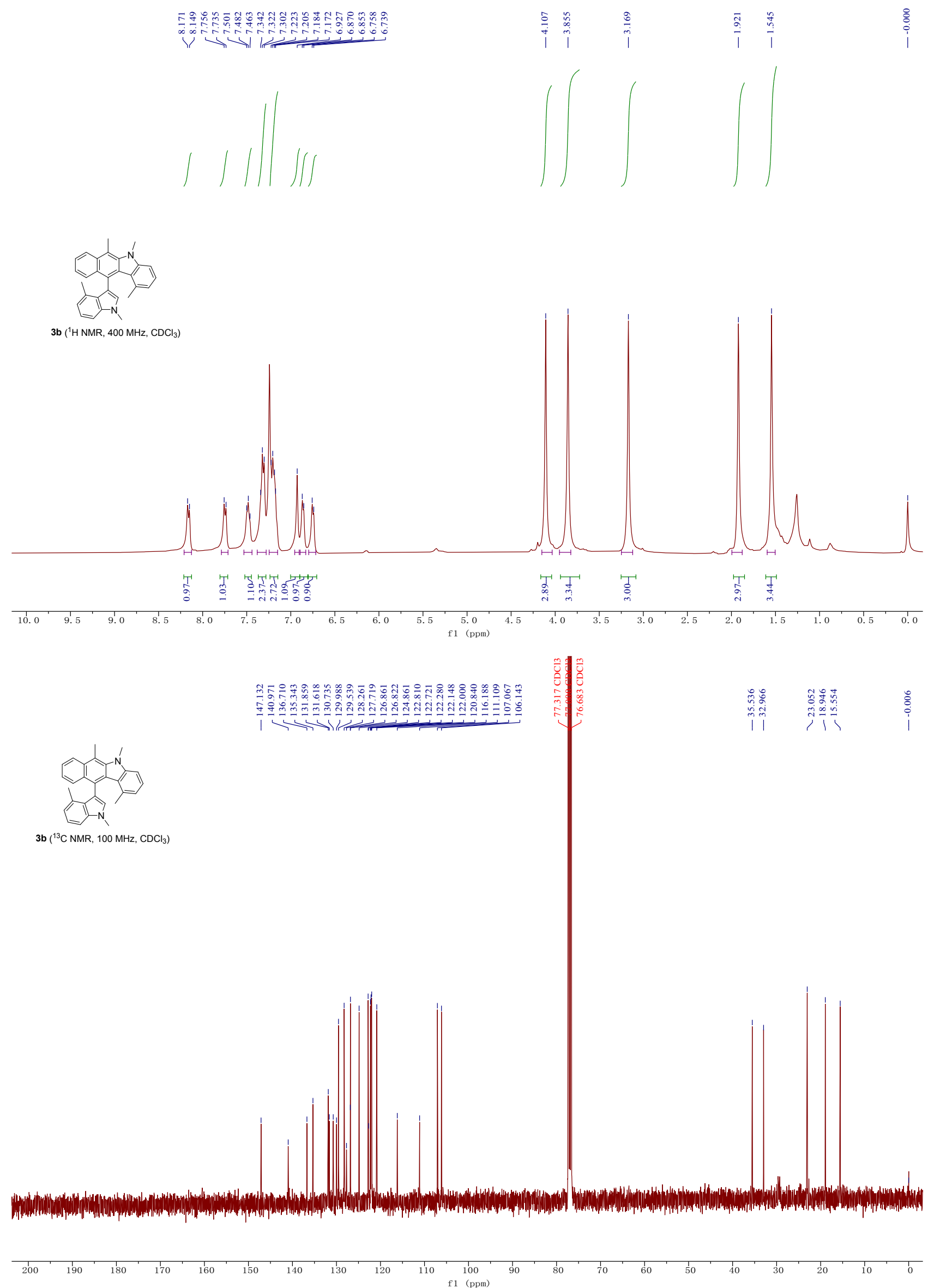


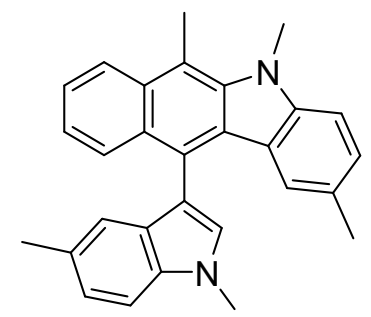

Compound 3c: Yield: $77 \mathrm{mg}, 96 \%$; a yellow solid; Mp: $>200{ }^{\circ} \mathrm{C}$; Eluent: PE/DCM =3/1. ${ }^{1} \mathrm{H}$ NMR $\left(400 \mathrm{MHz}, \mathrm{CDCl}_{3}, \mathrm{TMS}\right) 8.22(\mathrm{~d}, J=8.7 \mathrm{~Hz}, 1 \mathrm{H}), 7.91(\mathrm{~d}, J=8.7 \mathrm{~Hz}, 1 \mathrm{H}), 7.51(\mathrm{t}, J=7.6 \mathrm{~Hz}, 1 \mathrm{H})$, $7.41(\mathrm{~d}, J=8.4 \mathrm{~Hz}, 1 \mathrm{H}), 7.25(\mathrm{~d}, J=7.4 \mathrm{~Hz}, 1 \mathrm{H}), 7.18(\mathrm{~s}, 2 \mathrm{H}), 7.12$ (d, J=10.0 Hz, 2H), 6.91 (s, 1H), $6.76(\mathrm{~s}, 1 \mathrm{H}), 4.12(\mathrm{~s}, 3 \mathrm{H}), 3.97(\mathrm{~s}, 3 \mathrm{H}), 3.19$ (s, 3H), $2.25(\mathrm{~s}, 3 \mathrm{H}), 2.13(\mathrm{~s}, 3 \mathrm{H}) .{ }^{13} \mathrm{C}\left\{{ }^{1} \mathrm{H}\right\}-\mathrm{NMR}$ $\left(100 \mathrm{MHz}, \mathrm{CDCl}_{3}\right.$, TMS) $\delta_{\mathrm{C}} 144.0,140.5,135.6,132.1,129.4,128.9,128.7,128.4,127.74,127.65$, $127.4,125.6,124.7,123.7,123.5,122.7,122.0,120.1,111.9,111.6,108.9,107.8,34.7,33.1,21.35$, 21.31, 14.8; IR (neat): v 2985, 2917, 2903, 1601, 1490, 1337, 1079, 793, $753 \mathrm{~cm}^{-1}$; HRMS (EI-TOF) $\mathrm{m} / \mathrm{z}$ : $[\mathrm{M}]^{+}$Calcd for $\mathrm{C}_{29} \mathrm{H}_{26} \mathrm{~N}_{2}$ 402.2091; found 402.2098. 

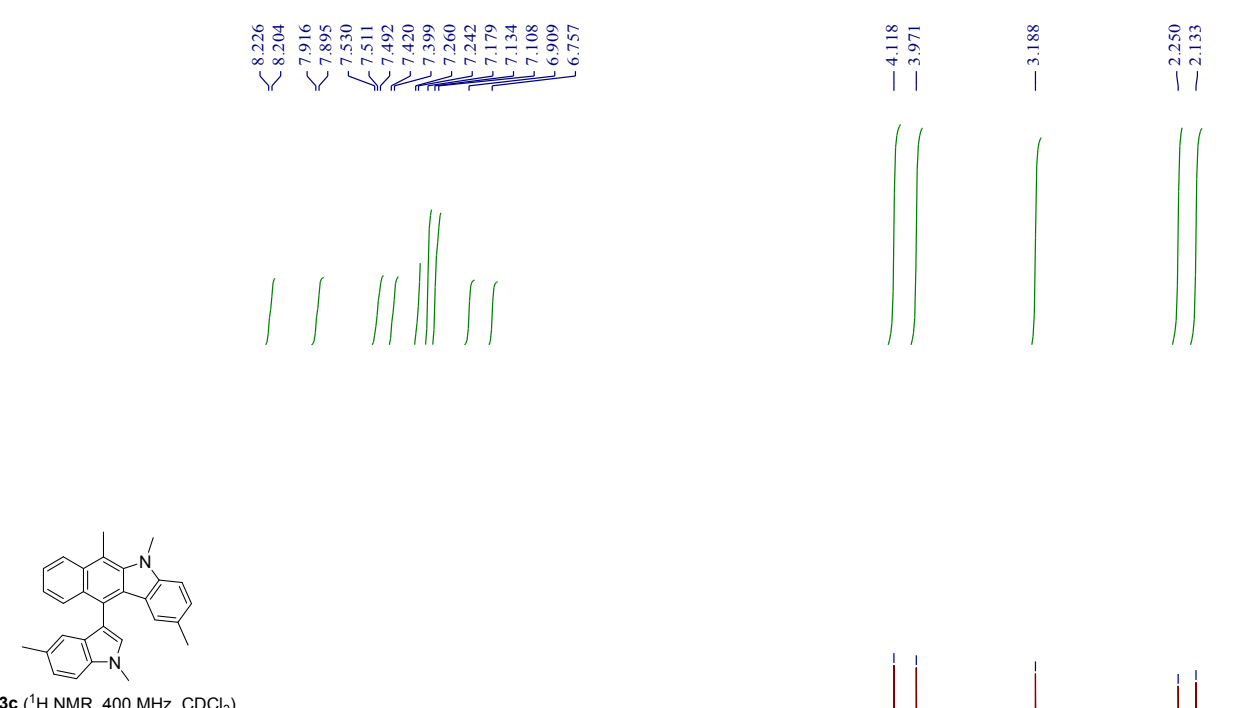

3c ( $\left.{ }^{1} \mathrm{H} \mathrm{NMR}, 400 \mathrm{MHz}, \mathrm{CDCl}_{3}\right)$

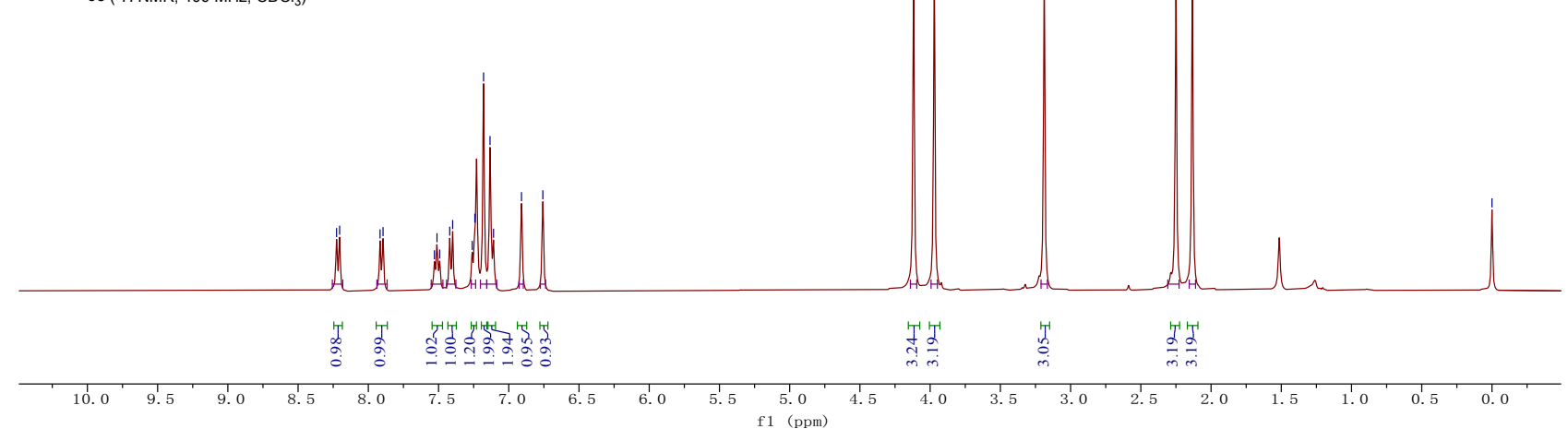

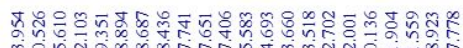

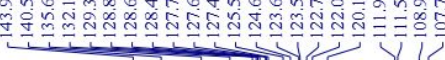

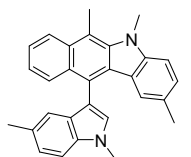

$3 c\left({ }^{13} \mathrm{C} \mathrm{NMR}, 100 \mathrm{MHz}, \mathrm{CDCl}_{3}\right)$
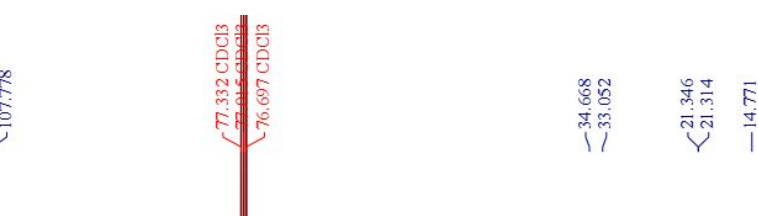

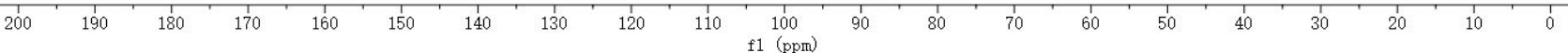




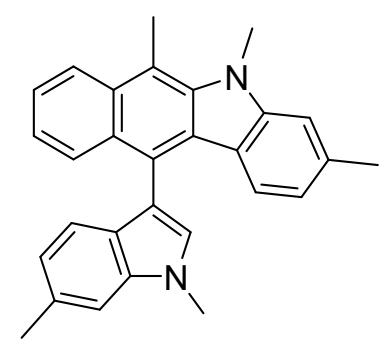

Compound 3d: Yield: $75 \mathrm{mg}$, 93\%; a yellow solid; Mp: $>200{ }^{\circ} \mathrm{C}$; Eluent: $\mathrm{PE} / \mathrm{DCM}=3 / 1 .{ }^{1} \mathrm{H}$ NMR (400 MHz, $\left.\mathrm{CDCl}_{3}, \mathrm{TMS}\right) \delta 8.20(\mathrm{~d}, J=8.6 \mathrm{~Hz}, 1 \mathrm{H}), 7.91(\mathrm{~d}, J=8.6 \mathrm{~Hz}, 1 \mathrm{H}), 7.54-7.45(\mathrm{~m}$, 1H), $7.30(\mathrm{~s}, 1 \mathrm{H}), 7.24(\mathrm{~d}, J=7.7 \mathrm{~Hz}, 1 \mathrm{H}), 7.08$ (d, $J=3.2 \mathrm{~Hz}, 2 \mathrm{H}), 7.01(\mathrm{~d}, J=8.0 \mathrm{~Hz}, 1 \mathrm{H}), 6.82$ $(\mathrm{dd}, J=8.1,3.1 \mathrm{~Hz}, 2 \mathrm{H}), 6.61(\mathrm{~d}, J=8.0 \mathrm{~Hz}, 1 \mathrm{H}), 4.10(\mathrm{~s}, 3 \mathrm{H}), 3.93(\mathrm{~s}, 3 \mathrm{H}), 3.17(\mathrm{~s}, 3 \mathrm{H}), 2.53(\mathrm{~s}$, 3H), $2.44(\mathrm{~s}, 3 \mathrm{H}) ;{ }^{13} \mathrm{C}\left\{{ }^{1} \mathrm{H}\right\}-\mathrm{NMR}\left(100 \mathrm{MHz}, \mathrm{CDCl}_{3}\right.$, TMS) $\delta_{\mathrm{C}} 146.0,140.2,137.5,136.9,131.9$, $131.6,129.4,127.7,127.2,126.5,125.6,124.5,124.0,122.9,122.7,122.0,121.3,121.1,120.2$, 120.0, 112.3, 111.5, 109.3, 108.4, 34.5, 33.0, 22.2, 21.9, 14.7; IR (neat): v 3024, 2919, 1596, 1472 , 1388, 1293, 799, $753 \mathrm{~cm}^{-1}$; HRMS (ESI-FTMS) m/z: [M+H] ${ }^{+} \mathrm{C}_{29} \mathrm{H}_{27} \mathrm{~N}_{2}$ 403.2169; found 403.2170. 

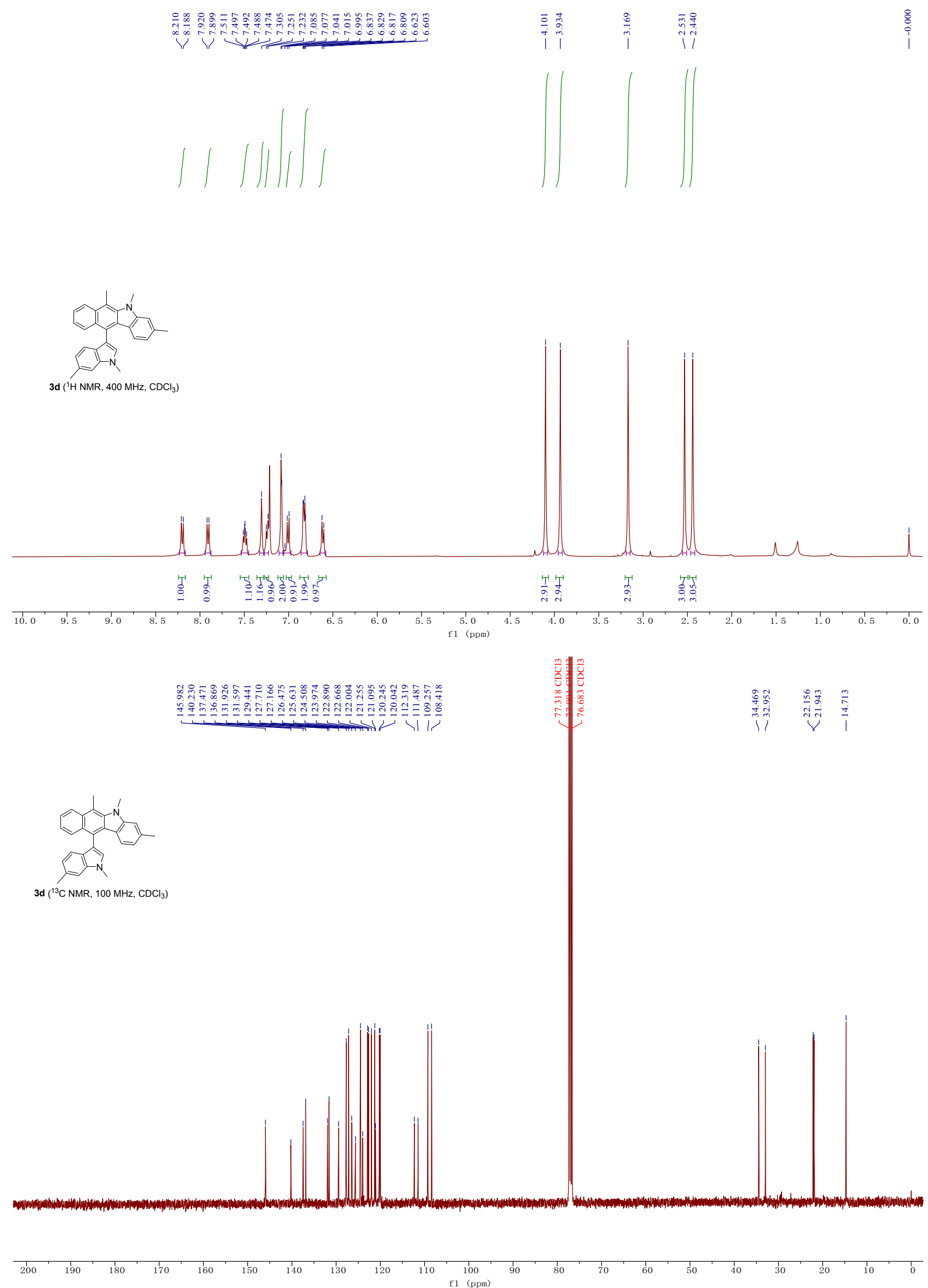


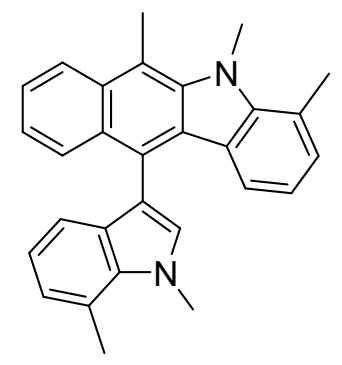

Compound 3e: Yield: $60 \mathrm{mg}$, 74\%; a yellow solid; Mp: $>200{ }^{\circ} \mathrm{C}$; Eluent: PE/DCM =3/1. ${ }^{1} \mathrm{H}$ NMR $\left(400 \mathrm{MHz}, \mathrm{CDCl}_{3}, \mathrm{TMS}\right) \delta 8.18(\mathrm{~d}, J=8.6 \mathrm{~Hz}, 1 \mathrm{H}), 7.86(\mathrm{~d}, J=8.6 \mathrm{~Hz}, 1 \mathrm{H}), 7.50(\mathrm{t}, J=7.6 \mathrm{~Hz}$, 1H), $7.26(\mathrm{~d}, J=7.6 \mathrm{~Hz}, 1 \mathrm{H}), 7.10$ (d, $J=7.2 \mathrm{~Hz}, 1 \mathrm{H}), 7.04(\mathrm{~s}, 1 \mathrm{H}), 6.97(\mathrm{~d}, J=7.5 \mathrm{~Hz}, 1 \mathrm{H}), 6.93$ $(\mathrm{d}, J=7.5 \mathrm{~Hz}, 1 \mathrm{H}), 6.87-6.78(\mathrm{~m}, 2 \mathrm{H}), 6.74(\mathrm{t}, J=7.5 \mathrm{~Hz}, 1 \mathrm{H}), 4.23$ (s, 3H), 4.01 (s, 3H), 3.09 (s, 3H), $2.92(\mathrm{~s}, 3 \mathrm{H}), 2.73(\mathrm{~s}, 3 \mathrm{H}) ;{ }^{13} \mathrm{C}\left\{{ }^{1} \mathrm{H}\right\}-\mathrm{NMR}\left(100 \mathrm{MHz}, \mathrm{CDCl}_{3}, \mathrm{TMS}\right) \delta_{\mathrm{C}} 147.7,144.8,135.8$, $132.4,130.3,129.9,129.8,129.7,127.3,126.8,125.9,124.8,124.5,124.1,122.9,122.5,121.2$, 121.14, 121.05, 120.2, 119.7, 118.8, 113.4, 112.1, 39.4, 37.0, 20.0, 19.9, 15.6; IR (neat): v 3063, 2966, 2908, 1594, 1470, 1383, 1117, $742 \mathrm{~cm}^{-1}$; HRMS (ESI-FTMS) m/z: $[\mathrm{M}+\mathrm{H}]^{+}$Calcd for $\mathrm{C}_{27} \mathrm{H}_{27} \mathrm{~N}_{2}$ 403.2169; found 403.2169. 


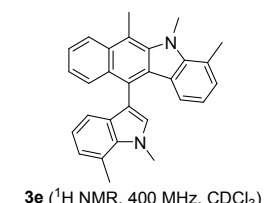

3e ( $\left.{ }^{1} \mathrm{H} \mathrm{NMR}, 400 \mathrm{MHz}, \mathrm{CDCl}_{3}\right)$
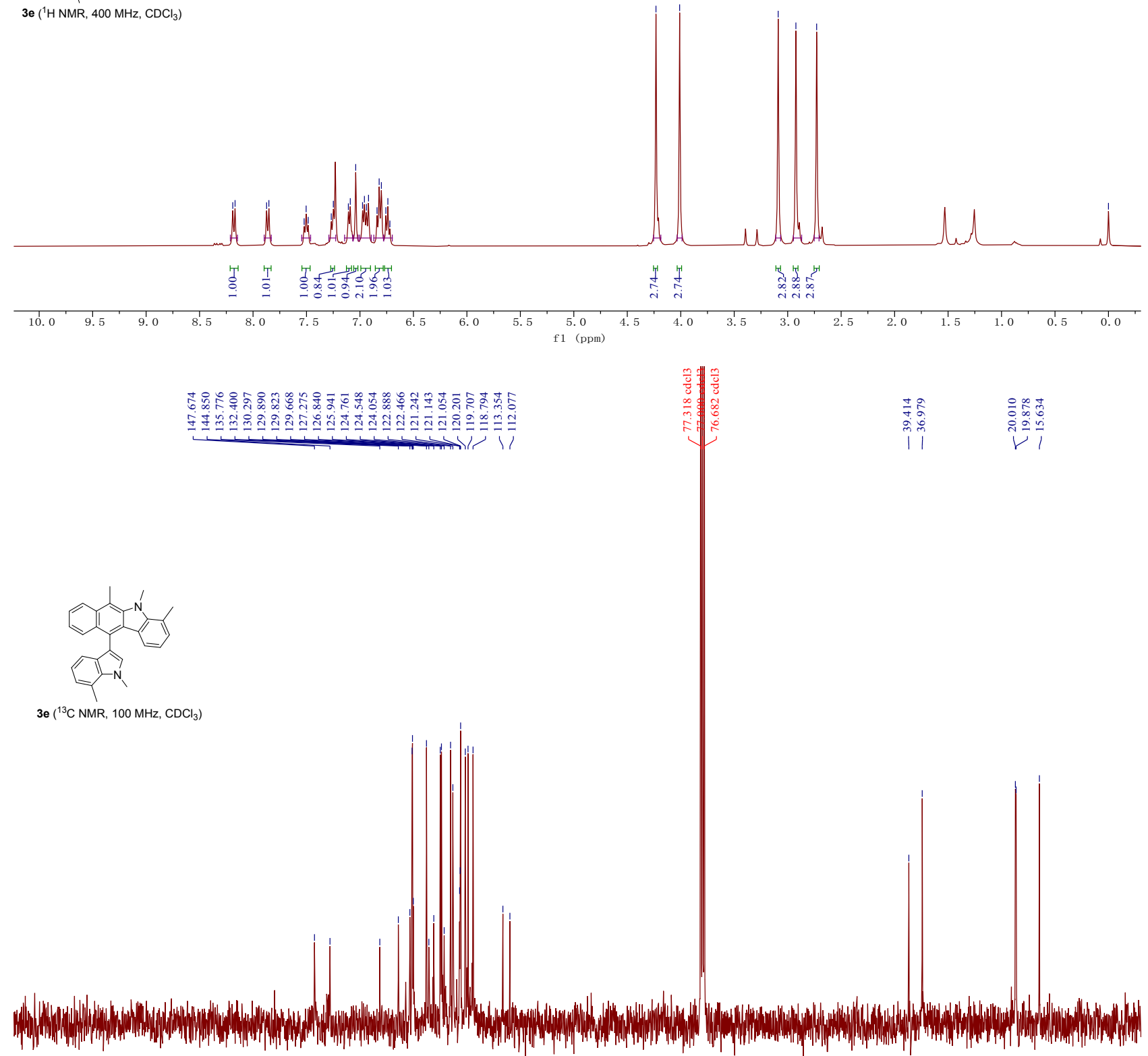

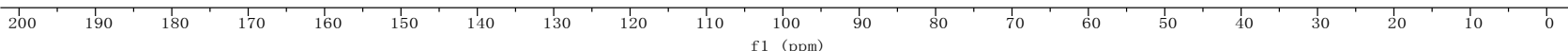




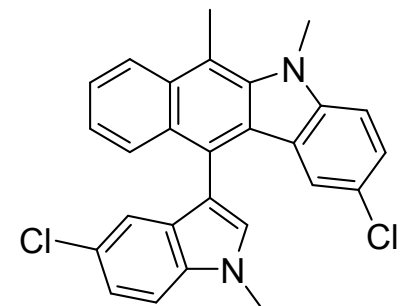

Compound 3f: Yield: $50 \mathrm{mg}, 56 \%$; a yellow solid; Mp: $>200{ }^{\circ} \mathrm{C}$ (this isolated product containing a small of impurity due to its poor solubility); Eluent: $\mathrm{PE} / \mathrm{EA}=1 / 1 .{ }^{1} \mathrm{H} \mathrm{NMR}\left(400 \mathrm{MHz}, \mathrm{CDCl}_{3}\right.$, TMS) $\delta 8.24(\mathrm{~d}, J=8.7 \mathrm{~Hz}, 1 \mathrm{H}), 7.83(\mathrm{~d}, J=8.7 \mathrm{~Hz}, 1 \mathrm{H}), 7.56(\mathrm{t}, J=7.6 \mathrm{~Hz}, 1 \mathrm{H}), 7.46(\mathrm{~d}, J=8.7$ $\mathrm{Hz}, 1 \mathrm{H}), 7.37-7.28(\mathrm{~m}, 3 \mathrm{H}), 7.23(\mathrm{~d}, J=5.0 \mathrm{~Hz}, 2 \mathrm{H}), 7.03(\mathrm{~d}, J=2.1 \mathrm{~Hz}, 1 \mathrm{H}), 6.81(\mathrm{~d}, J=2.1 \mathrm{~Hz}$, 1H), $4.16(\mathrm{~s}, 3 \mathrm{H}), 4.02(\mathrm{~s}, 3 \mathrm{H}), 3.21(\mathrm{~s}, 3 \mathrm{H}) ;{ }^{13} \mathrm{C}\left\{{ }^{1} \mathrm{H}\right\}-\mathrm{NMR}\left(100 \mathrm{MHz}, \mathrm{CD}_{2} \mathrm{Cl}_{2}, \mathrm{TMS}\right): \delta_{\mathrm{C}}$ 144.6, $142.7,140.8,136.3,133.1,130.5,129.9,127.4,127.2,126.0,125.7,124.9,124.4,124.2,123.5$, 123.1, 122.9, 122.8, 119.7, 113.3, 112.0, 111.5, 109.8, 35.2, 33.8, 15.0; IR (neat): v 2598, 2920, 2850, 1592, 1471, 1261, 790, $743 \mathrm{~cm}-1$; HRMS (ESI-FTMS) m/z: $[\mathrm{M}+\mathrm{H}]^{+}$Calcd for $\mathrm{C}_{27} \mathrm{H}_{21} \mathrm{~N}_{2} \mathrm{Cl}_{2}$ 443.1076; found 443.1078. 


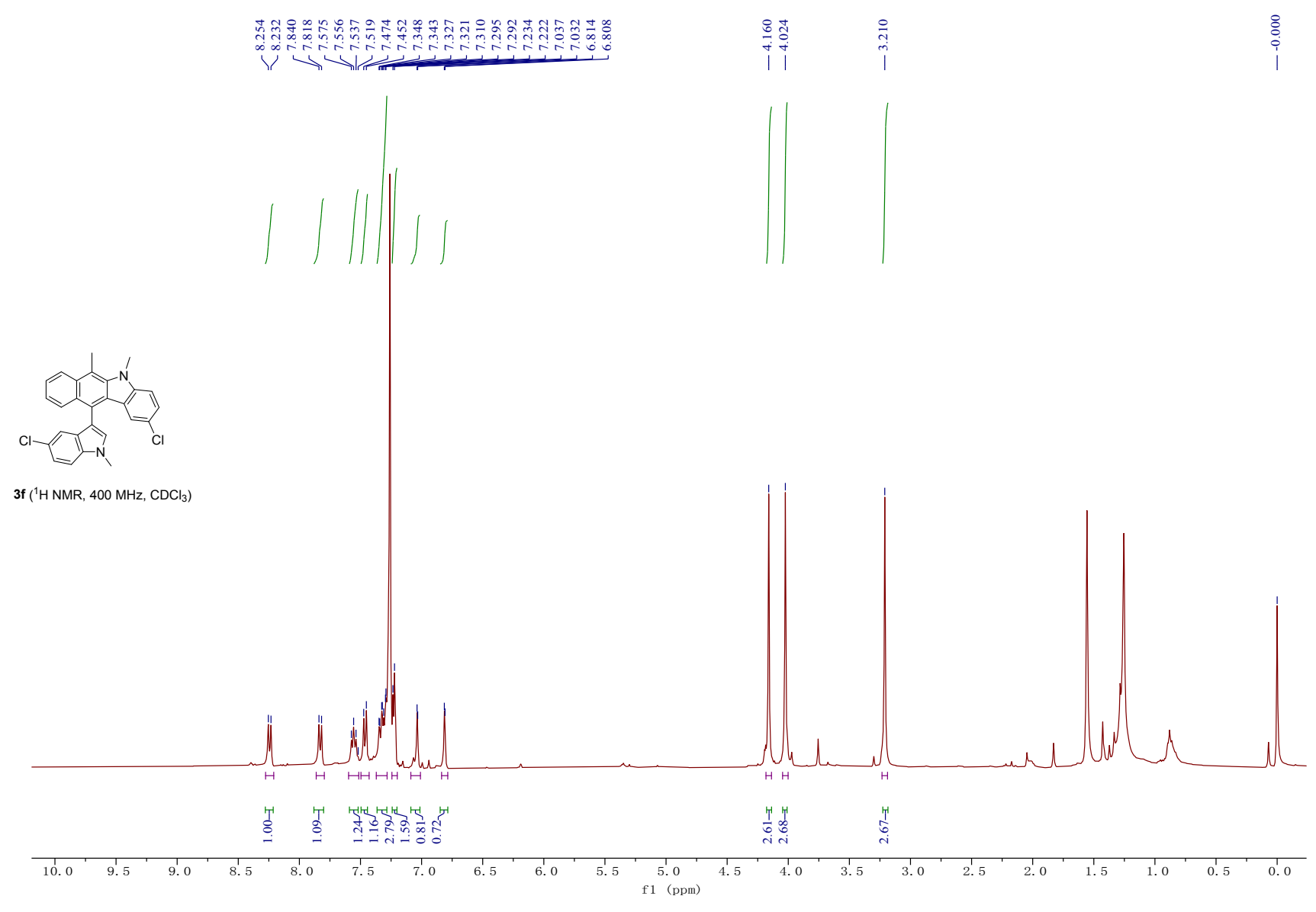

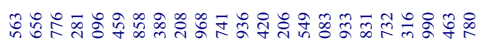

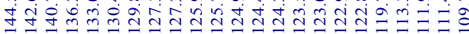
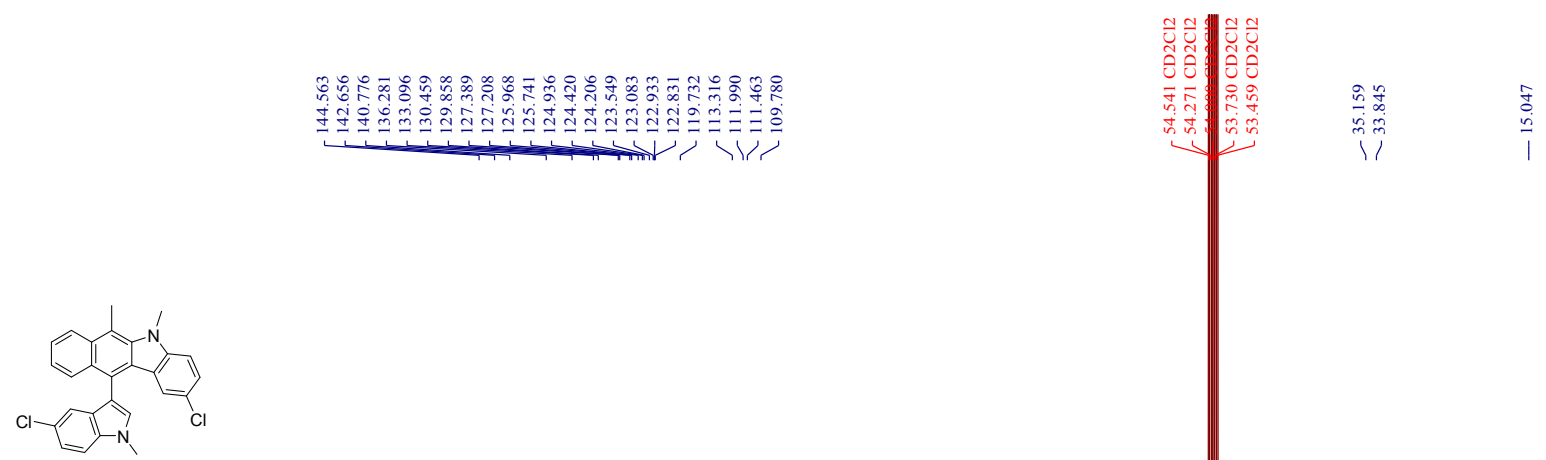

3f $\left({ }^{13} \mathrm{C} \mathrm{NMR}, 100 \mathrm{MHz}, \mathrm{CD}_{2} \mathrm{Cl}_{2}\right)$

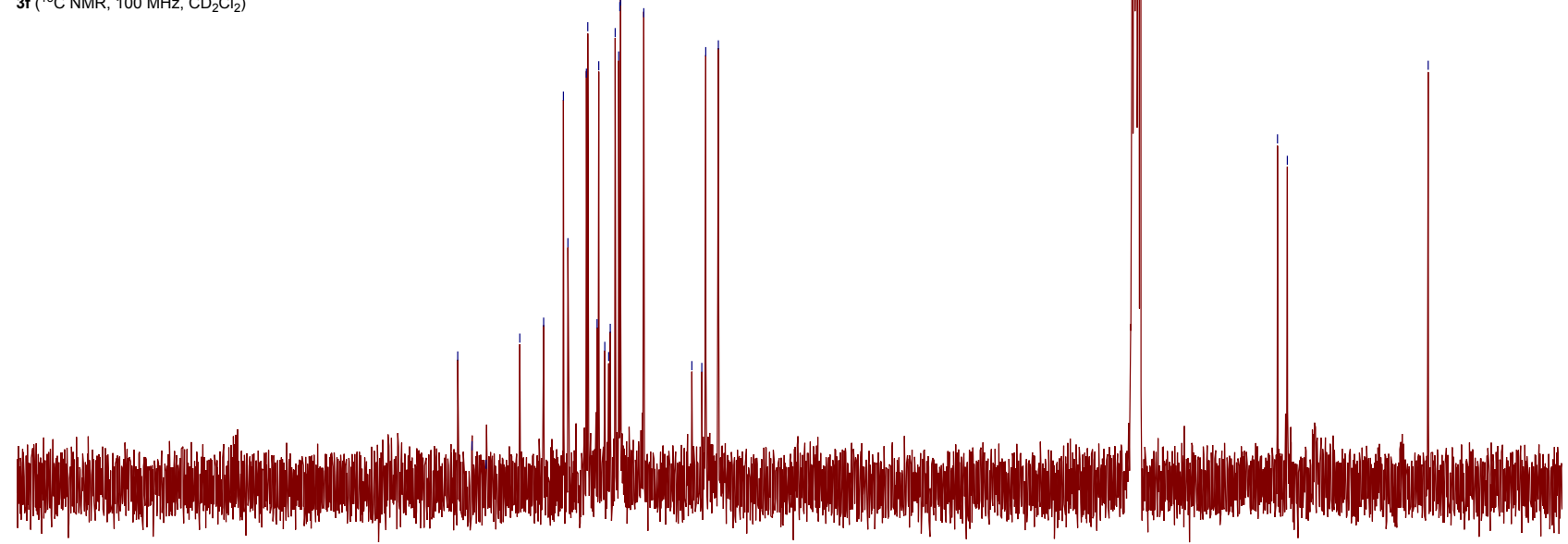

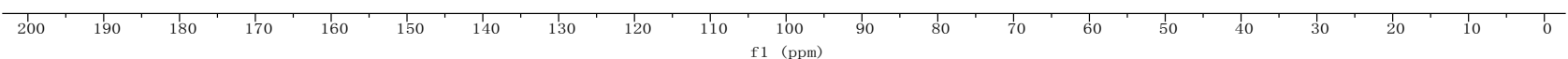




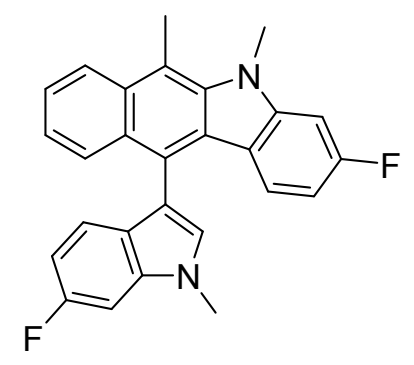

Compound 3g: Yield: 40 mg, 49\%; a yellow solid; Mp: $>200{ }^{\circ} \mathrm{C}$; Eluent: PE/DCM =2/1. ${ }^{1} \mathrm{H}$ NMR $\left(400 \mathrm{MHz}, \mathrm{CDCl}_{3}, \mathrm{TMS}\right) \delta 8.22(\mathrm{~d}, J=8.7 \mathrm{~Hz}, 1 \mathrm{H}), 7.88(\mathrm{~d}, J=8.7 \mathrm{~Hz}, 1 \mathrm{H}), 7.53$ (td, $J=6.8,3.4$ $\mathrm{Hz}, 1 \mathrm{H}), 7.32-7.24(\mathrm{~m}, 1 \mathrm{H}), 7.19$ (dd, $J=9.8,2.3 \mathrm{~Hz}, 1 \mathrm{H}), 7.13$ (s, 1H), $7.01-6.91$ (m, 2H), 6.75 (ddd, $J=11.6,8.7,4.0 \mathrm{~Hz}, 2 \mathrm{H}), 6.50(\mathrm{td}, J=9.0,2.3 \mathrm{~Hz}, 1 \mathrm{H}), 4.09$ (s, 3H), 3.94 (s, 3H), 3.18 (s, $3 \mathrm{H}) ;{ }^{13} \mathrm{C}\left\{{ }^{1} \mathrm{H}\right\}$-NMR $\left(100 \mathrm{MHz}, \mathrm{CDCl}_{3}\right.$, TMS) $\delta_{\mathrm{C}} 162.7(\mathrm{~d}, J=243.2 \mathrm{~Hz}), 160.1(\mathrm{~d}, J=238.0 \mathrm{~Hz})$, 146.6, 146.5, 140.2, 137.1, 137.0, 131.9, 129.5, 128.5 (d, $J=3.7 \mathrm{~Hz}), 126.9,124.84,124.79,124.0$ $(\mathrm{d}, J=10.2 \mathrm{~Hz}), 123.2,122.9,122.5,121.2(\mathrm{~d}, J=10.2 \mathrm{~Hz}), 119.4,112.4,112.1,108.4(\mathrm{~d}, J=24.6$ $\mathrm{Hz}), 106.2$ (d, $J=23.3 \mathrm{~Hz}), 95.9$ (d, $J=26.3 \mathrm{~Hz}), 95.3$ (d, $J=26.9 \mathrm{~Hz}), 34.6,33.2,14.6$; ${ }^{19} \mathrm{~F}$ NMR $\left(376 \mathrm{MHz}, \mathrm{CDCl}_{3}\right) \delta-113.6,-120.5$. IR (neat): v 3066, 2924, 1620, 1591, 1477, 1387, 1102, 821, $753 \mathrm{~cm}^{-1}$; HRMS (EI-TOF) m/z: [M] Calcd for $\mathrm{C}_{27} \mathrm{H}_{20} \mathrm{~N}_{2} \mathrm{~F}_{2} 410.1589$; found 410.1594 . 


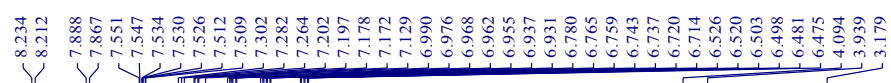
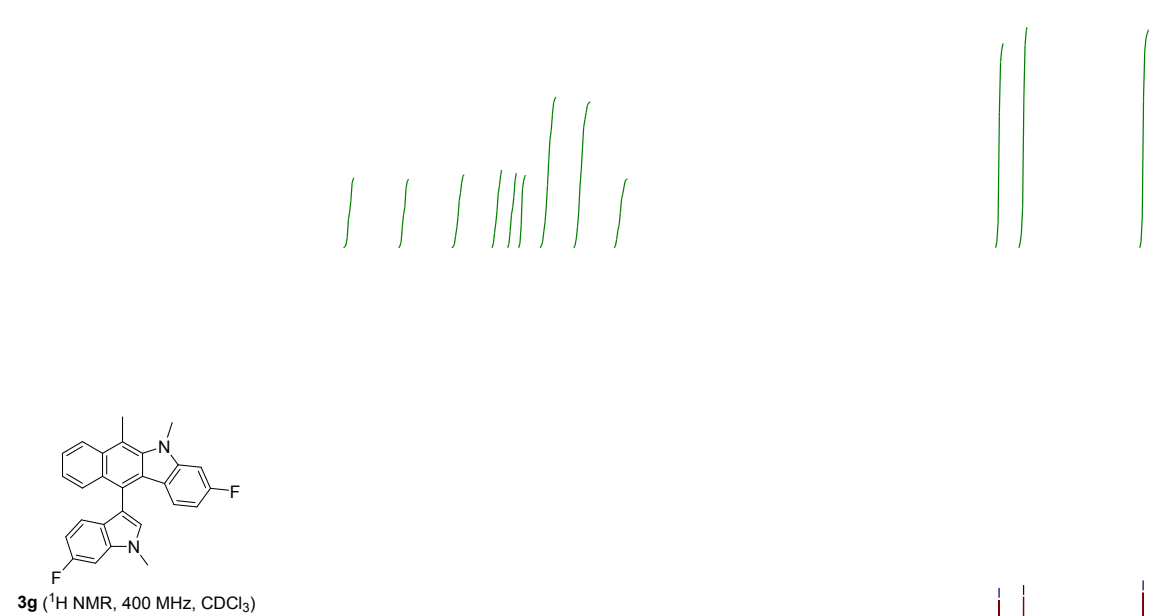

Haduha

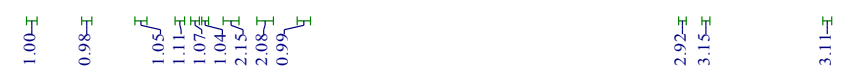

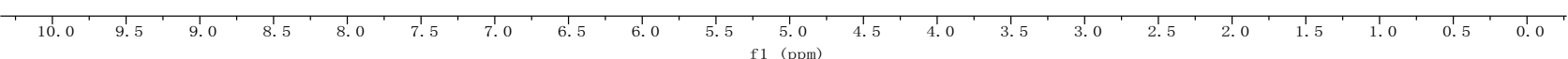

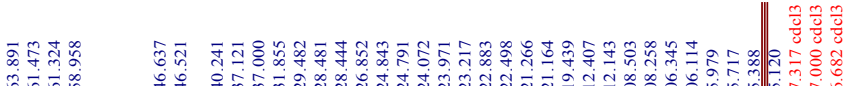

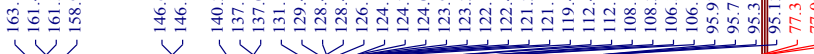

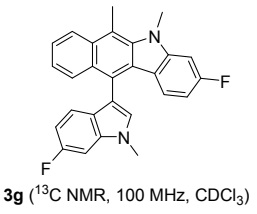

3g $\left({ }^{13} \mathrm{C} \mathrm{NMR}, 100 \mathrm{MHz}, \mathrm{CDCl}_{3}\right)$

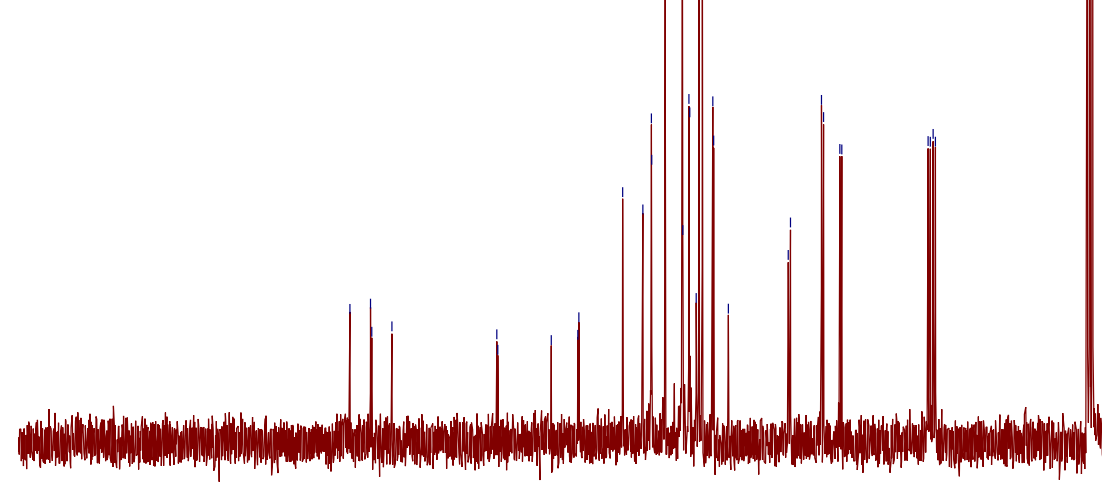

$\longmapsto$

$\mathrm{HH}$

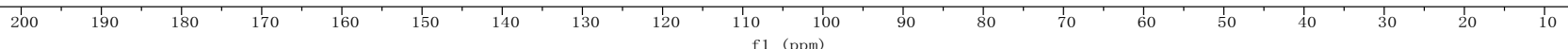




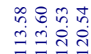

Yi
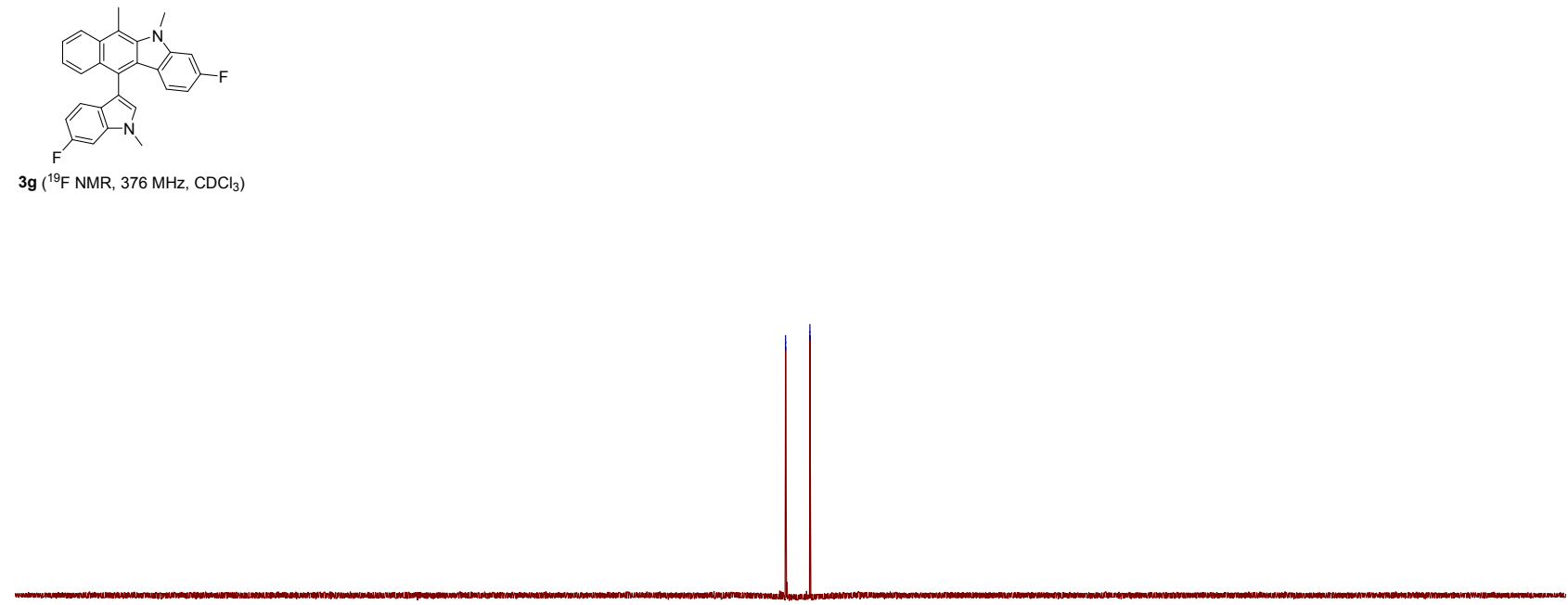

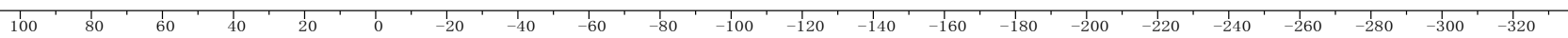




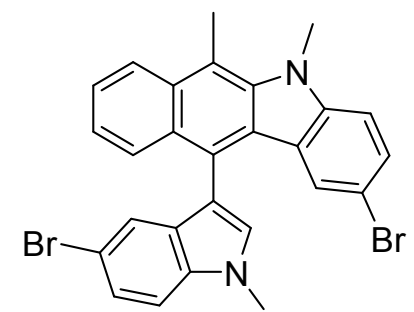

Compound 3h: Yield: $44 \mathrm{mg}$, 41\%; a yellow solid; Mp: $>200{ }^{\circ} \mathrm{C}$; Eluent: PE/EA = 2/1 (this isolated product containing a small of impurity due to its poor solubility). ${ }^{1} \mathrm{H}$ NMR (400 MHz, $\left.\mathrm{CD}_{2} \mathrm{Cl}_{2}, \mathrm{TMS}\right) \delta 8.18(\mathrm{~d}, J=8.8 \mathrm{~Hz}, 1 \mathrm{H}), 7.73(\mathrm{~d}, J=8.8 \mathrm{~Hz}, 1 \mathrm{H}), 7.47(\mathrm{ddd}, J=8.6,6.7,1.4 \mathrm{~Hz}$, $1 \mathrm{H}), 7.39(\mathrm{dd}, J=8.7,1.7 \mathrm{~Hz}, 2 \mathrm{H}), 7.31(\mathrm{dd}, J=8.7,1.9 \mathrm{~Hz}, 1 \mathrm{H}), 7.25-7.12(\mathrm{~m}, 3 \mathrm{H}), 7.03(\mathrm{~d}, J=$ $1.9 \mathrm{~Hz}, 1 \mathrm{H}), 6.85(\mathrm{~d}, J=2.0 \mathrm{~Hz}, 1 \mathrm{H}), 4.07(\mathrm{~s}, 3 \mathrm{H}), 3.94(\mathrm{~s}, 3 \mathrm{H}), 3.13(\mathrm{~s}, 3 \mathrm{H}) ;{ }^{13} \mathrm{C}\left\{{ }^{1} \mathrm{H}\right\}-\mathrm{NMR}(100$ $\mathrm{MHz}, \mathrm{CD}_{2} \mathrm{Cl}_{2}$, TMS) $\delta_{\mathrm{C}} 144.3,139.9,135.9,132.5,129.9,129.7,129.28,129.25,126.8,125.4$, $125.2,125.0,124.8,124.3,123.8,123.0,122.5,122.2,113.0,112.7,111.3,111.0,109.7,34.5,33.2$, 14.4; IR (neat): $v$ 3076, 2914, 1600, 1473, 1287, 1122, 791, $749 \mathrm{~cm}^{-1}$; HRMS (EI-TOF) m/z: [M] Calcd for $\mathrm{C}_{27} \mathrm{H}_{20} \mathrm{~N}_{2} \mathrm{Br}_{2}$ 529.9988; found 529.9994. 

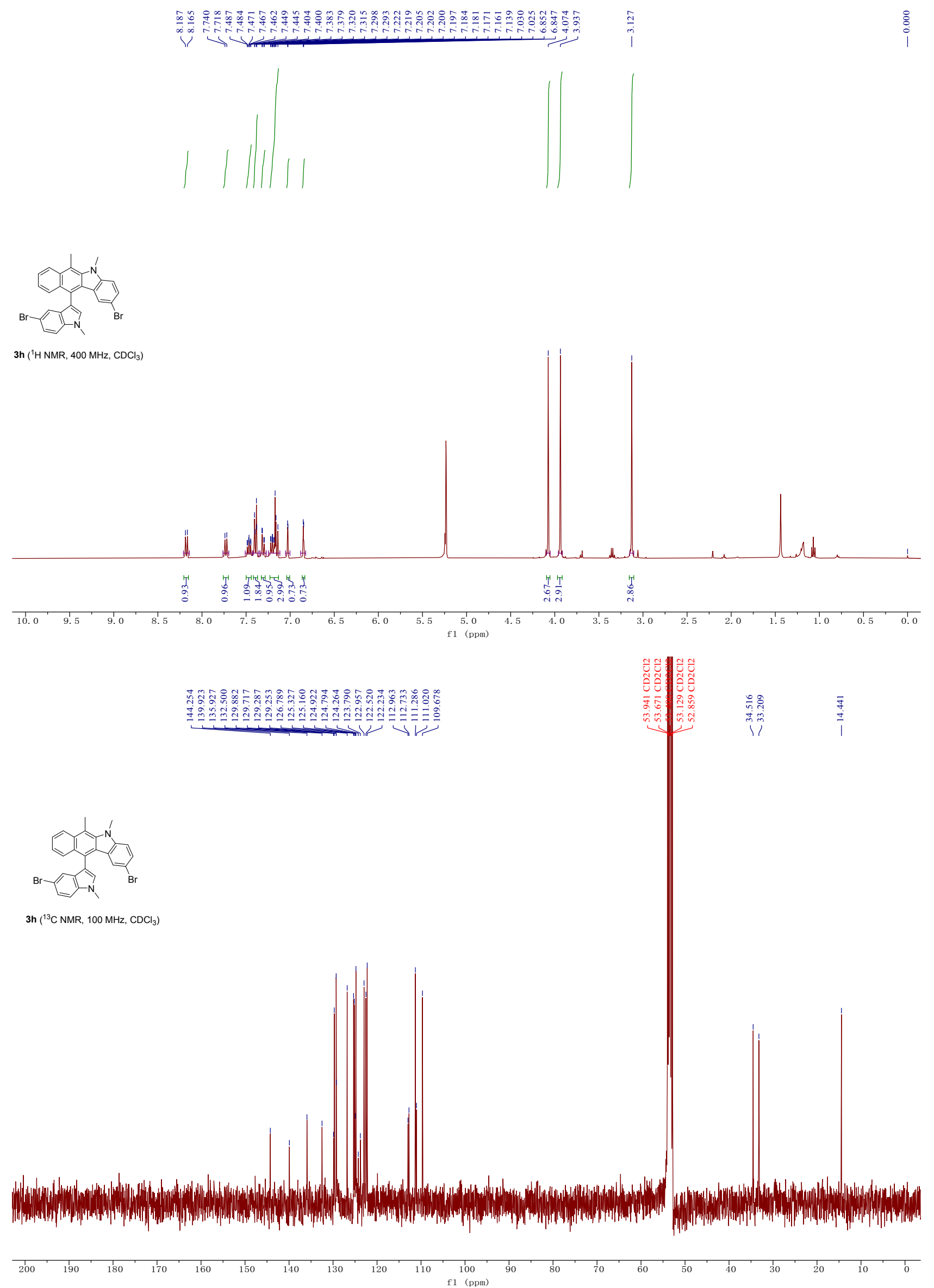


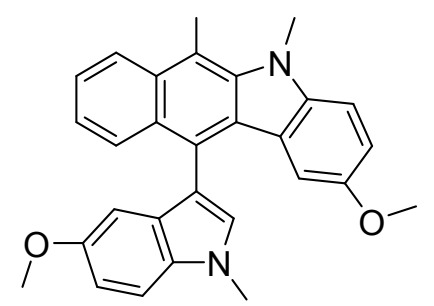

Compound 3i: Yield: $56 \mathrm{mg}, 65 \%$; a yellow solid; Mp: $>200{ }^{\circ} \mathrm{C}$; Eluent: PE/DCM =2/1. ${ }^{1} \mathrm{H}$ NMR $\left(400 \mathrm{MHz}, \mathrm{CDCl}_{3}, \mathrm{TMS}\right) \delta 8.23(\mathrm{~d}, J=8.7 \mathrm{~Hz}, 1 \mathrm{H}), 8.03(\mathrm{~d}, J=8.5 \mathrm{~Hz}, 1 \mathrm{H}), 7.53(\mathrm{t}, J=7.7 \mathrm{~Hz}$, 1H), 7.39 (d, $J=8.9 \mathrm{~Hz}, 1 \mathrm{H}), 7.29$ (d, $J=7.4 \mathrm{~Hz}, 1 \mathrm{H}), 7.19$ (d, $J=8.7 \mathrm{~Hz}, 1 \mathrm{H}), 7.14$ (s, 1H), 6.96 $(\mathrm{m}, 2 \mathrm{H}), 6.55(\mathrm{~s}, 1 \mathrm{H}), 6.37(\mathrm{~s}, 1 \mathrm{H}), 4.12(\mathrm{~s}, 3 \mathrm{H}), 3.95(\mathrm{~s}, 3 \mathrm{H}), 3.51(\mathrm{~s}, 3 \mathrm{H}), 3.29(\mathrm{~s}, 3 \mathrm{H}), 3.20(\mathrm{~s}, 3 \mathrm{H})$; ${ }^{13} \mathrm{C}\left\{{ }^{1} \mathrm{H}\right\}$-NMR (100 MHz, $\mathrm{CDCl}_{3}$, TMS) $\delta_{\mathrm{C}} 154.3,152.9,140.8,140.6,132.3,132.1,129.1,128.9$, $127.3,125.5,124.8,124.5,123.7,122.7,122.0,115.3,112.7,111.9,111.8,110.0,108.6,106.7$, 101.7, 55.7, 55.1, 34.7, 33.2, 14.7; IR (neat): $v$ 2914, 2825, 1491, 1450, 1220, 1140, 798, $755 \mathrm{~cm}^{-1}$; HRMS (ESI-TOF) m/z: [M+H $]^{+}$Calcd for $\mathrm{C}_{29} \mathrm{H}_{26} \mathrm{~N}_{2} \mathrm{O}_{2}$ 434.1989; found 434.1995. 

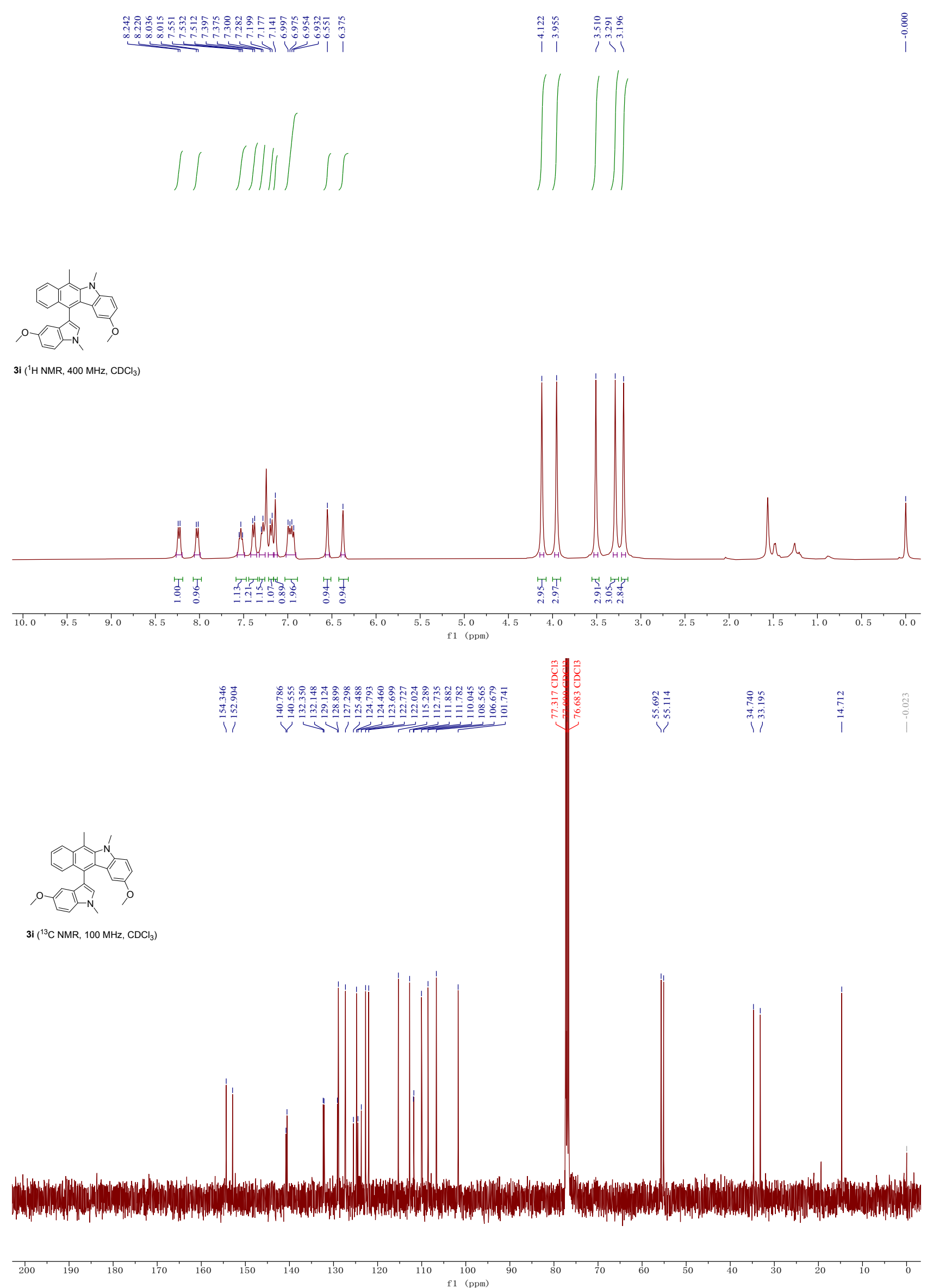


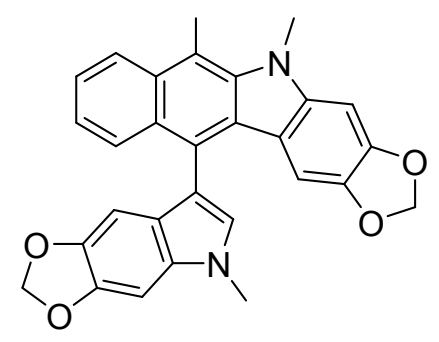

Compound 3j: Yield: $50 \mathrm{mg}$, 54\%; a yellow solid; Mp: $>200{ }^{\circ} \mathrm{C}$; Eluent: PE/DCM =1/1. ${ }^{1} \mathrm{H}$ NMR $\left(400 \mathrm{MHz}, \mathrm{CD}_{2} \mathrm{Cl}_{2}\right) \delta 8.21(\mathrm{~d}, J=8.7 \mathrm{~Hz}, 1 \mathrm{H}), 7.85(\mathrm{~d}, J=8.7 \mathrm{~Hz}, 1 \mathrm{H}), 7.48(\mathrm{t}, J=7.7 \mathrm{~Hz}, 1 \mathrm{H})$, $7.24(\mathrm{t}, J=7.7 \mathrm{~Hz}, 1 \mathrm{H}), 7.05(\mathrm{~s}, 1 \mathrm{H}), 7.00(\mathrm{~s}, 1 \mathrm{H}), 6.86(\mathrm{~s}, 1 \mathrm{H}), 6.35(\mathrm{~s}, 1 \mathrm{H}), 6.32(\mathrm{~s}, 1 \mathrm{H}), 5.89$ (s, 2H), $5.87(\mathrm{~s}, 2 \mathrm{H}), 4.09(\mathrm{~s}, 3 \mathrm{H}), 3.92(\mathrm{~s}, 3 \mathrm{H}), 3.17(\mathrm{~s}, 3 \mathrm{H}) ;{ }^{13} \mathrm{C}\left\{{ }^{1} \mathrm{H}\right\}-\mathrm{NMR}\left(100 \mathrm{MHz}, \mathrm{CD}_{2} \mathrm{Cl}_{2}\right) \delta_{\mathrm{C}}$ $147.8,145.2,143.0,141.8,141.3,140.2,132.4,131.2,129.2,126.8,126.6,125.4,124.2,122.8$, $122.5,122.4,122.0,115.9,112.2,112.0,102.4,101.0,100.7,98.2,90.4,90.2,34.8,33.3,14.5$; IR (neat): $v$ 3074, 2898, 1629, 1595, 1472, 1231, 943, 833, $754 \mathrm{~cm}^{-1}$; HRMS (ESI-FTMS) m/z: $[\mathrm{M}+\mathrm{H}]^{+}$Calcd for $\mathrm{C}_{29} \mathrm{H}_{23} \mathrm{~N}_{2} \mathrm{O}_{4} 463.1652$; found 463.1652 . 


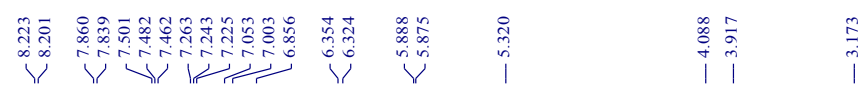
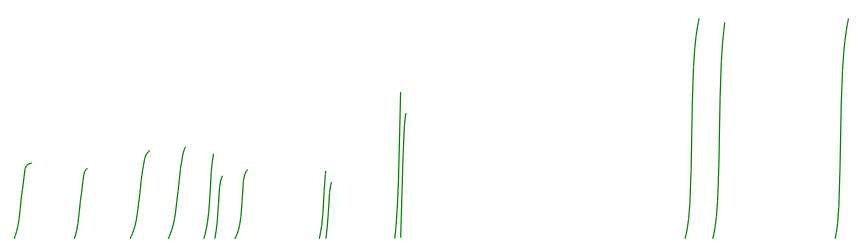
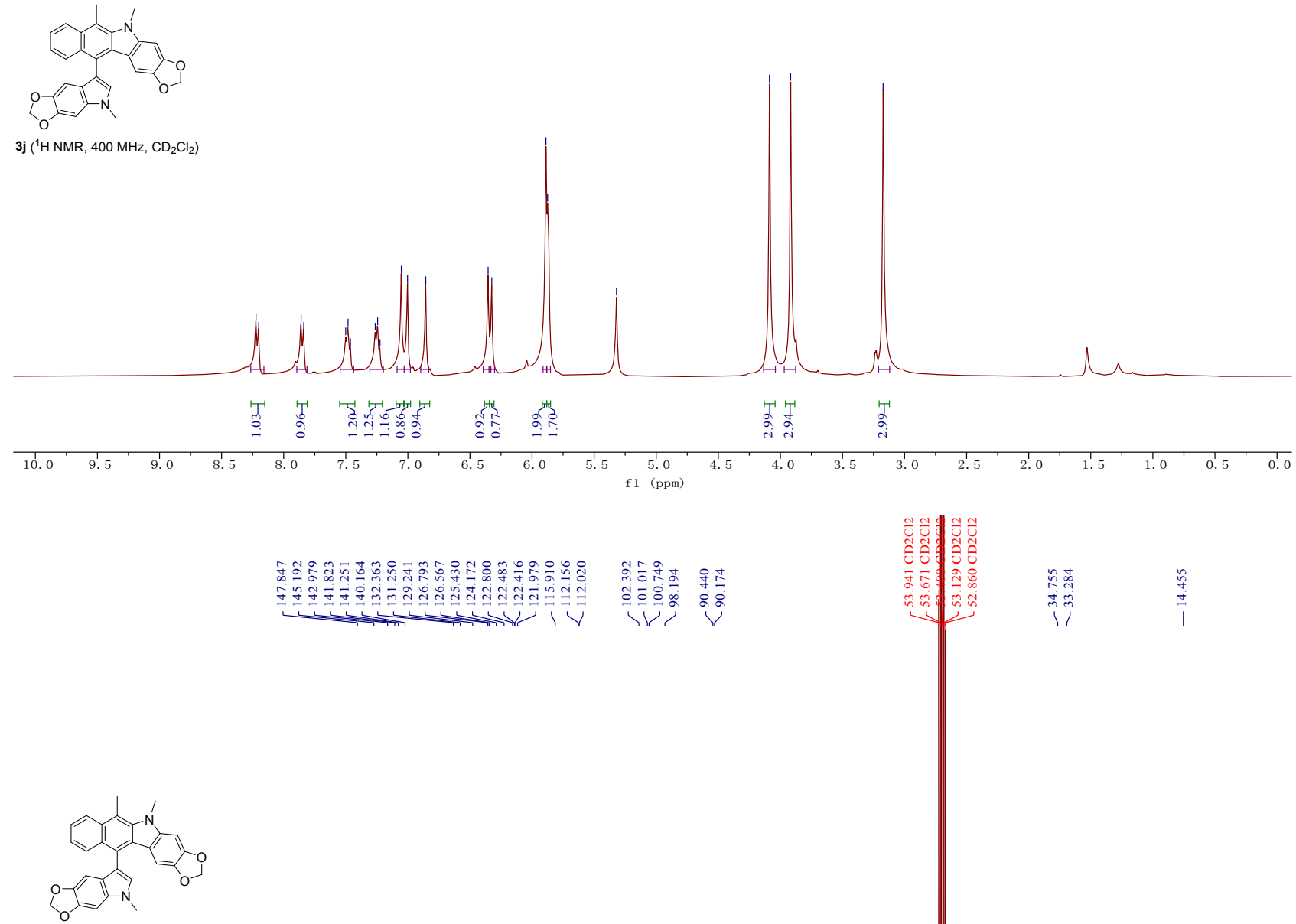

3j $\left.{ }^{13} \mathrm{C} \mathrm{NMR}, 100 \mathrm{MHz}, \mathrm{CD}_{2} \mathrm{Cl}_{2}\right)$
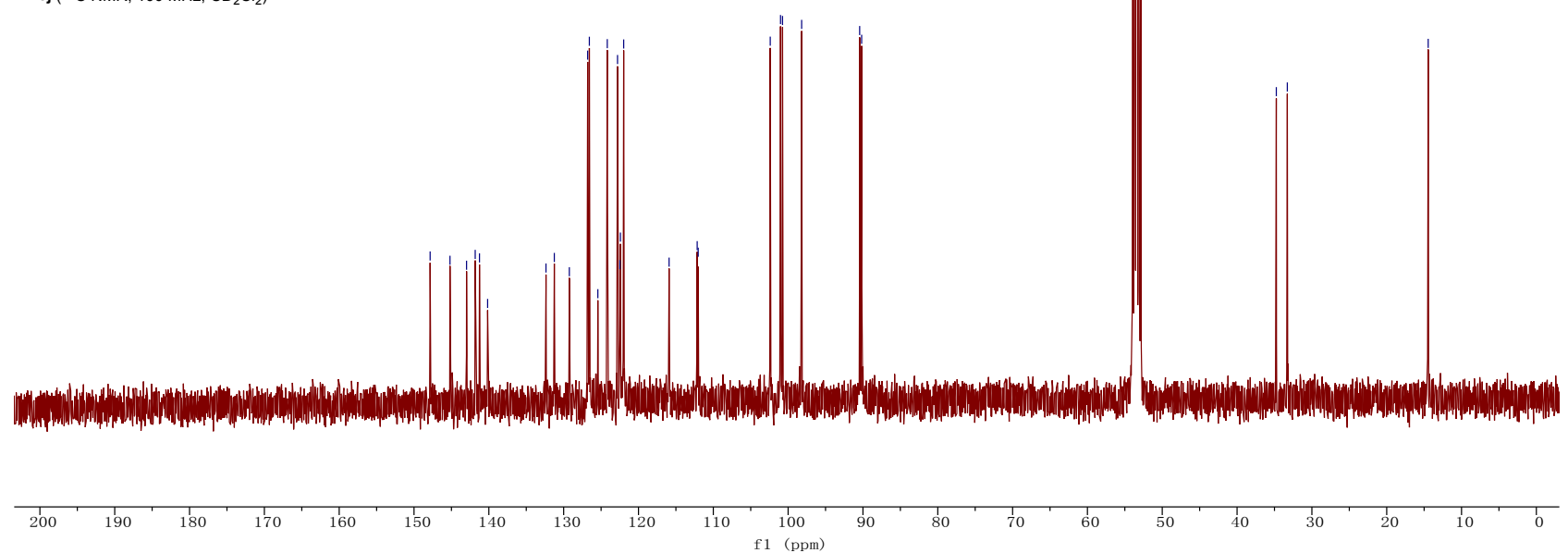


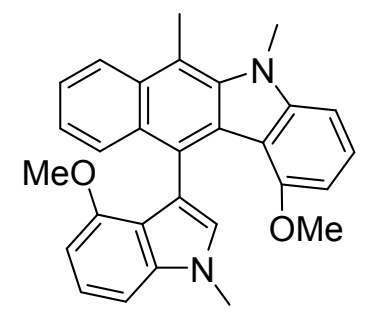

Compound 3k: Yield: $49 \mathrm{mg}, 50 \%(\mathrm{dr}=1: 1)$; a yellow solid; $\mathrm{Mp}: 154-157{ }^{\circ} \mathrm{C}$; Eluent: $\mathrm{PE} / \mathrm{DCM}=$ 1/1. ${ }^{1} \mathrm{H}$ NMR (400 MHz, $\mathrm{CDCl}_{3}$, TMS) $\delta 8.32(\mathrm{~d}, J=8.7 \mathrm{~Hz}, 1 \mathrm{H}), 8.12(\mathrm{~d}, J=8.9 \mathrm{~Hz}, 1 \mathrm{H}), 7.98$ $(\mathrm{dd}, J=8.7,0.7 \mathrm{~Hz}, 1 \mathrm{H}), 7.69$ (d, $J=8.1 \mathrm{~Hz}, 1 \mathrm{H}), 7.47-7.41(\mathrm{~m}, 1 \mathrm{H}), 7.41-7.32(\mathrm{~m}, 2 \mathrm{H}), 7.29$ (t, $J=8.1 \mathrm{~Hz}, 1 \mathrm{H}), 7.23(\mathrm{ddd}, J=8.6,6.5,1.2 \mathrm{~Hz}, 1 \mathrm{H}), 7.19$ (d, $J=1.8 \mathrm{~Hz}, 1 \mathrm{H}), 7.18-7.14$ (m, 2H), $7.08-7.01(\mathrm{~m}, 2 \mathrm{H}), 6.89-6.82(\mathrm{~m}, 4 \mathrm{H}), 6.77(\mathrm{~s}, 1 \mathrm{H}), 6.68(\mathrm{dd}, J=8.1,0.8 \mathrm{~Hz}, 1 \mathrm{H}), 6.49-6.42$ (m, 2H), $6.31(\mathrm{dd}, J=8.1,0.8 \mathrm{~Hz}, 1 \mathrm{H}), 4.04(\mathrm{~s}, 3 \mathrm{H}), 4.03(\mathrm{~s}, 3 \mathrm{H}), 3.82(\mathrm{~s}, 3 \mathrm{H}), 3.79$ (s, 3H), 3.45 (s, 3H), 3.27 (s, 3H), $3.26(\mathrm{~s}, 6 \mathrm{H}), 3.11(\mathrm{~s}, 3 \mathrm{H}), 2.84(\mathrm{~s}, 3 \mathrm{H}) ;{ }^{13} \mathrm{C}\left\{{ }^{1} \mathrm{H}\right\}-\mathrm{NMR}\left(100 \mathrm{MHz}, \mathrm{CDCl}_{3}, \mathrm{TMS}\right)$ $\delta_{\mathrm{C}} 155.9,155.27,155.26,155.1,147.9,146.9,140.2,139.6,138.4,138.3,134.0,131.4,130.5$, $128.8,128.6,128.4,127.94,127.86,127.7,127.3,126.1$, 126.0, 124.34, 124.30, 124.1, 123.8, 122.8, $122.1,121.8,121.7,121.4,121.3,120.6,115.8,112.4,111.9,111.0,110.4,110.1,102.5,102.1$, 101.3, 101.1, 101.0, 100.4, 100.2, 55.7, 55.4, 55.3, 54.4, 35.3, 33.1, 32.8, 32.4, 18.8, 15.2; IR (neat): v 2927, 2833, 1581, 1494, 1259, 1075, 750, $731 \mathrm{~cm}^{-1}$; HRMS (ESI-FTMS) m/z: [M+H] Calcd for 435.2067; found 435.2063. 

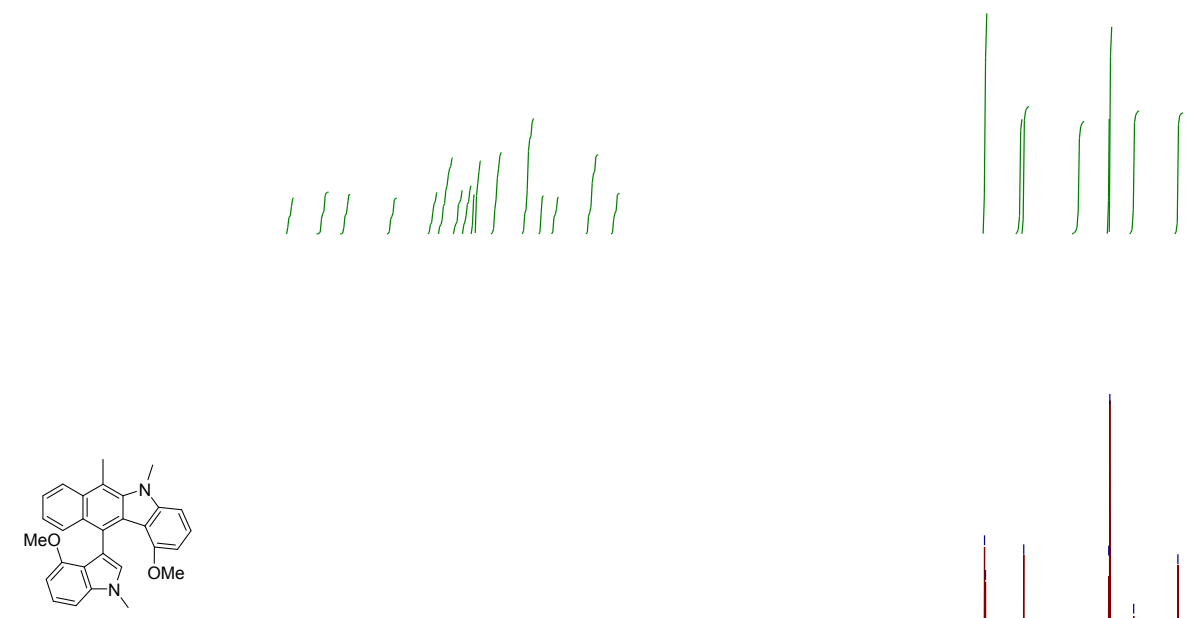

3k ( $\left.{ }^{1} \mathrm{H} \mathrm{NMR}, 400 \mathrm{MHz}, \mathrm{CDCl}_{3}\right)$
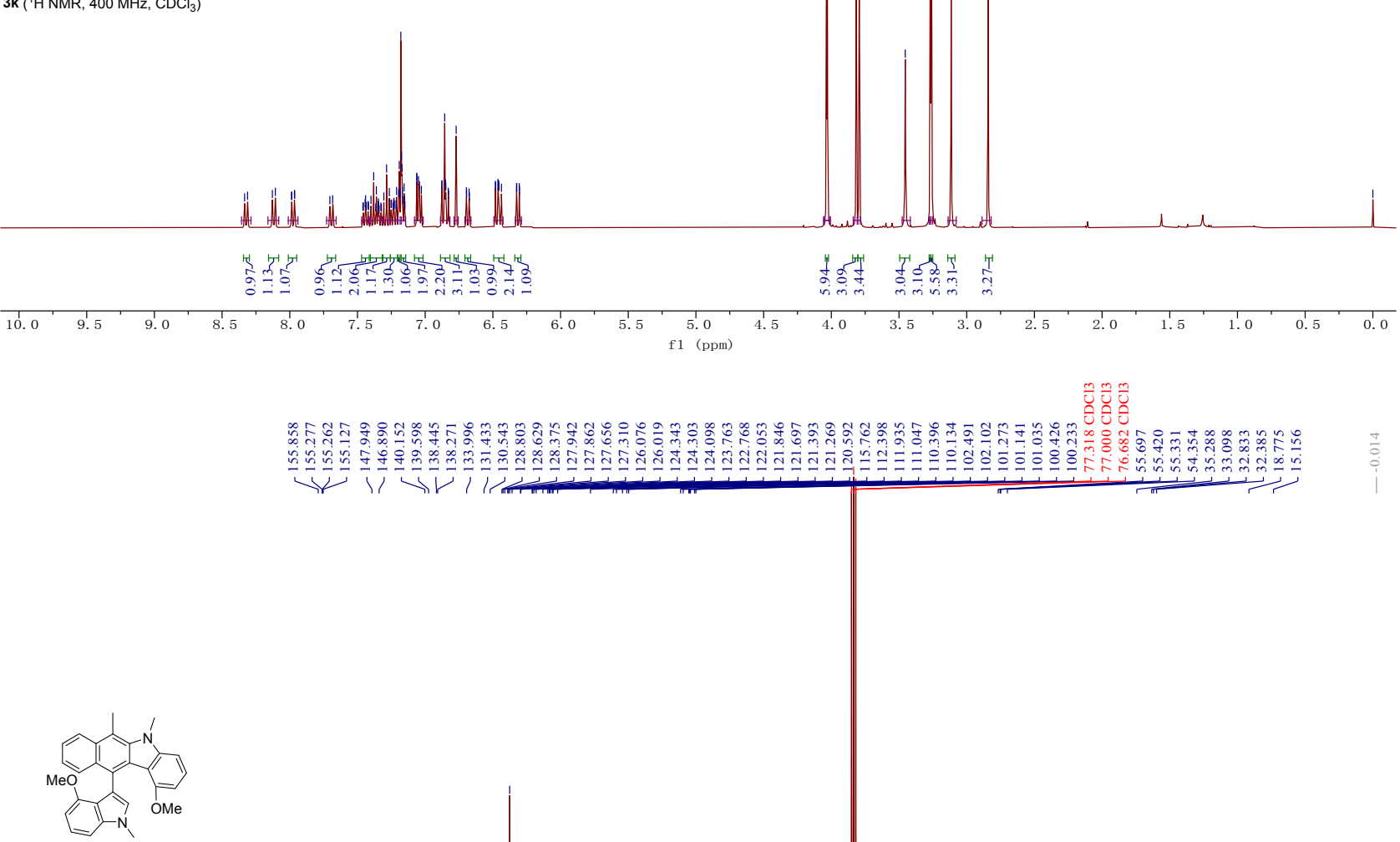

$3 \mathbf{3}\left({ }^{13} \mathrm{C} \mathrm{NMR}, 100 \mathrm{MHz}, \mathrm{CDCl}_{3}\right)$

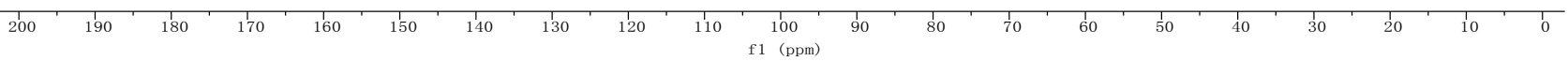




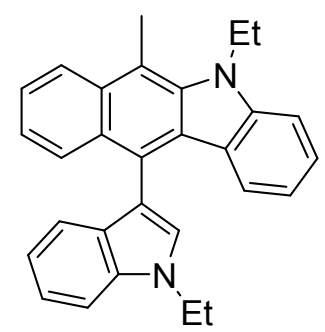

Compound 3I: Yield: $47 \mathrm{mg}$, 58\%; a yellow solid; Mp: 105-107 ${ }^{\circ} \mathrm{C}$; Eluent: PE/EA = 5/1. ${ }^{1} \mathrm{H}$ NMR (400 MHz, $\left.\mathrm{CDCl}_{3}, \mathrm{TMS}\right) \delta 8.23(\mathrm{~d}, J=8.7 \mathrm{~Hz}, 1 \mathrm{H}), 7.91(\mathrm{~d}, J=8.7 \mathrm{~Hz}, 1 \mathrm{H}), 7.55-7.46(\mathrm{~m}, 2 \mathrm{H})$, $7.38-7.22(\mathrm{~m}, 5 \mathrm{H}), 7.16-7.11(\mathrm{~m}, 1 \mathrm{H}), 6.97(\mathrm{ddd}, J=8.0,6.7,1.0 \mathrm{~Hz}, 1 \mathrm{H}), 6.92-6.88(\mathrm{~m}, 1 \mathrm{H})$, $6.76(\mathrm{ddd}, J=8.0,6.7,1.4 \mathrm{~Hz}, 1 \mathrm{H}), 4.58(\mathrm{qd}, J=7.1,1.8 \mathrm{~Hz}, 2 \mathrm{H}), 4.31$ (qd, $J=7.1,4.6 \mathrm{~Hz}, 2 \mathrm{H})$, $3.13(\mathrm{~s}, 3 \mathrm{H}), 1.56(\mathrm{t}, J=7.1 \mathrm{~Hz}, 3 \mathrm{H}), 1.50(\mathrm{t}, J=7.1 \mathrm{~Hz}, 3 \mathrm{H}) ;{ }^{13} \mathrm{C}\left\{{ }^{1} \mathrm{H}\right\}-\mathrm{NMR}\left(100 \mathrm{MHz}, \mathrm{CDCl}_{3}\right.$, TMS) $\delta_{\mathrm{C}} 144.5,138.8,136.1,132.1,129.3,128.7,127.3,126.7,126.6,125.8,124.74,124.67,123.7$, 123.2, 122.7, 122.1, 121.7, 120.7, 119.4, 118.7, 112.6, 111.3, 109.4, 108.0, 41.1, 40.8, 15.9, 15.2, 14.3; IR (neat): v 3068, 2972, 1592, 1376, 1343, 1269, 748, $737 \mathrm{~cm}^{-1}$; HRMS (ESI-FTMS) m/z: $[\mathrm{M}+\mathrm{H}]^{+}$Calcd for $\mathrm{C}_{29} \mathrm{H}_{27} \mathrm{~N}_{2}$ 403.2169; found 403.2168. 

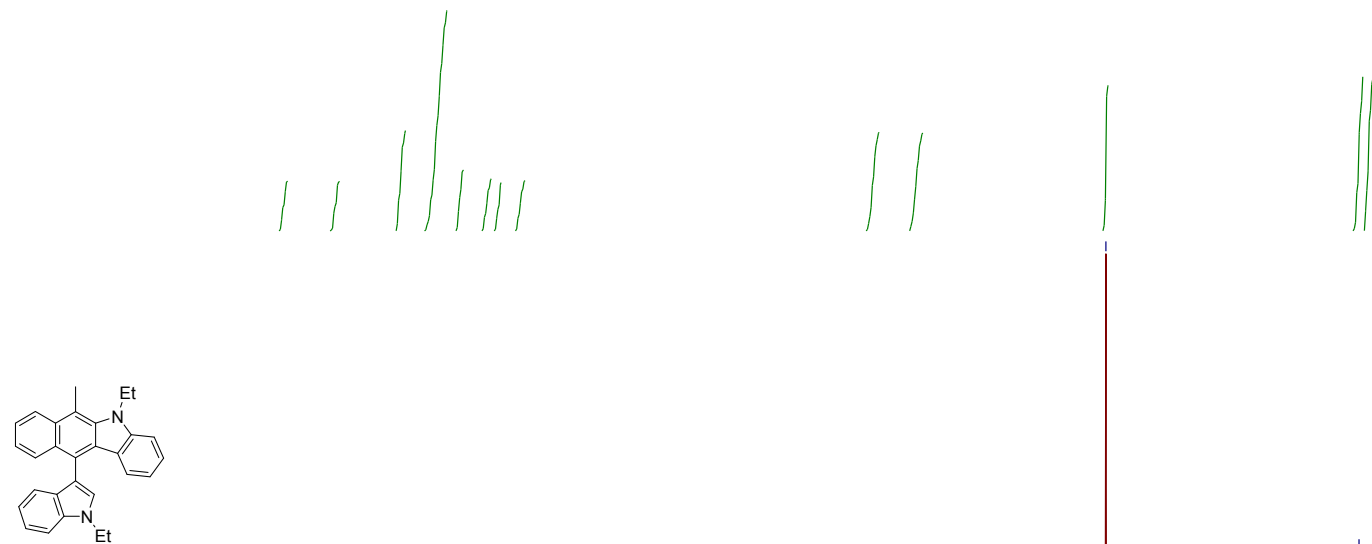

31 ( $\left.{ }^{1} \mathrm{H} \mathrm{NMR}, 400 \mathrm{MHz}, \mathrm{CDCl}_{3}\right)$
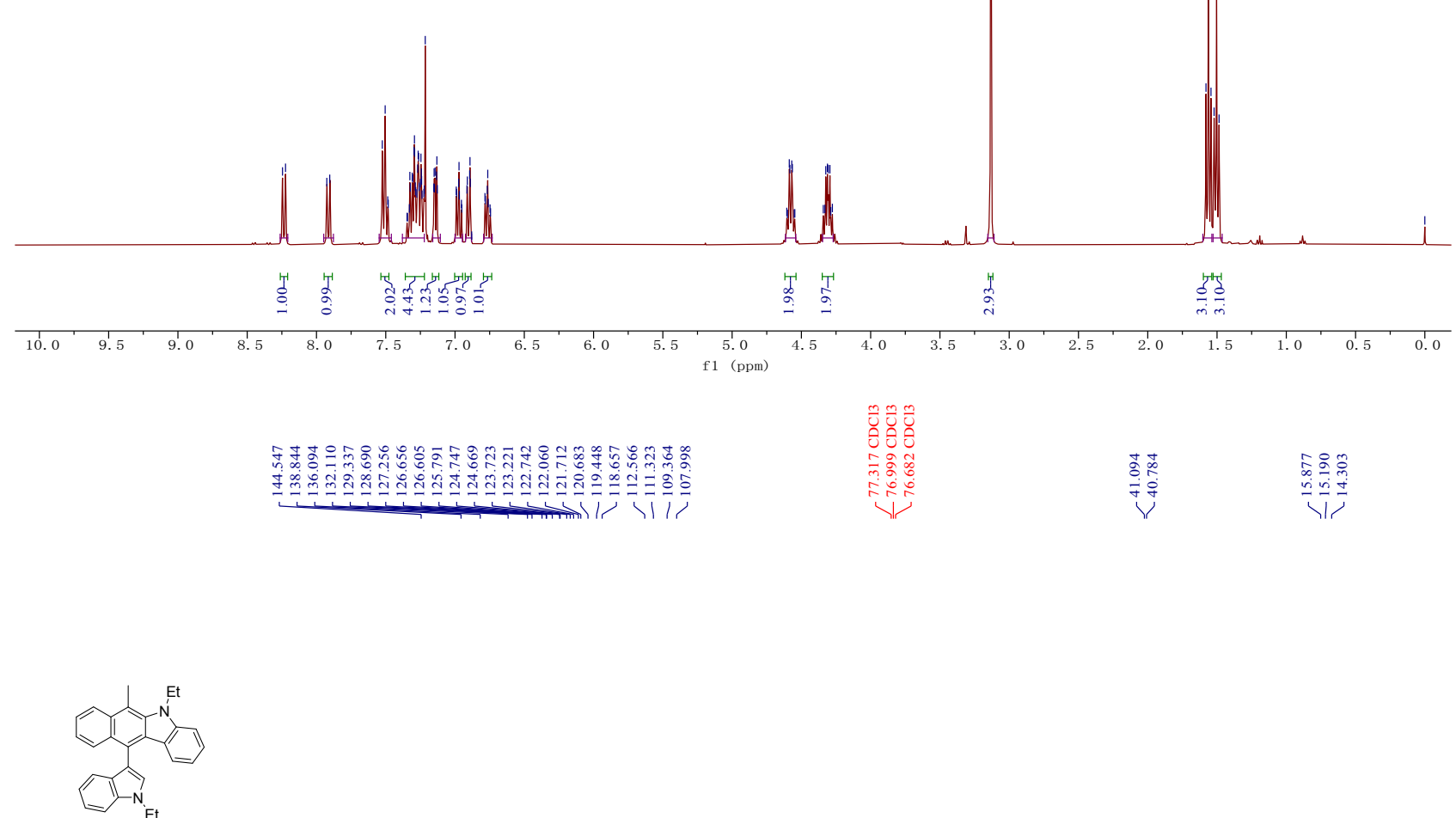

3l $\left({ }^{13} \mathrm{C} \mathrm{NMR}, 100 \mathrm{MHz}, \mathrm{CDCl}_{3}\right)$
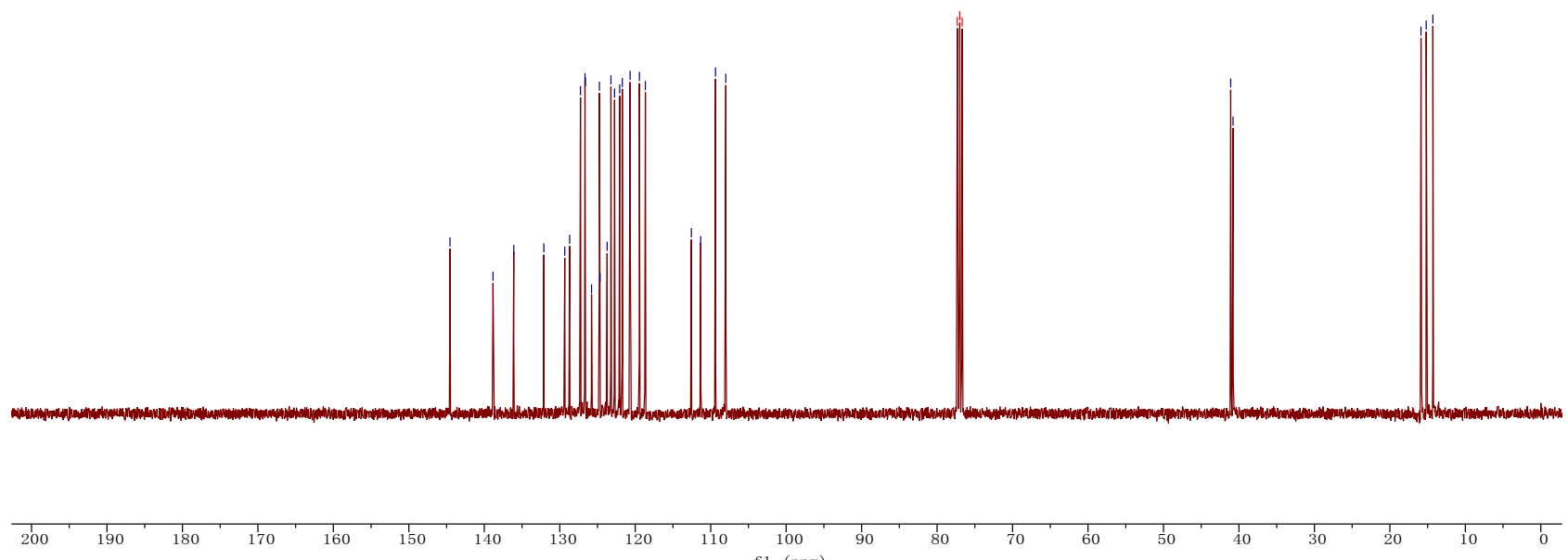


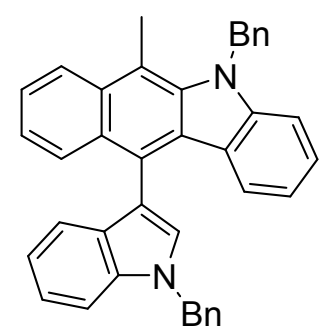

Compound 3m: Yield: 34 mg, 32\%; a yellow solid; Mp: $>200{ }^{\circ} \mathrm{C}$; Eluent: PE/EA = 8/1. ${ }^{1} \mathrm{H}$ NMR (400 MHz, $\left.\mathrm{CDCl}_{3}, \mathrm{TMS}\right) \delta 8.14(\mathrm{~d}, J=8.6 \mathrm{~Hz}, 1 \mathrm{H}), 7.94(\mathrm{~d}, J=8.6 \mathrm{~Hz}, 1 \mathrm{H}), 7.52-7.44(\mathrm{~m}, 2 \mathrm{H})$, $7.36-7.21(\mathrm{~m}, 14 \mathrm{H}), 7.19(\mathrm{~d}, J=8.0 \mathrm{~Hz}, 1 \mathrm{H}), 7.09$ (d, $J=8.1 \mathrm{~Hz}, 1 \mathrm{H}), 7.02-6.97(\mathrm{~m}, 2 \mathrm{H}), 6.75(\mathrm{t}$, $J=7.5 \mathrm{~Hz}, 1 \mathrm{H}), 5.69(\mathrm{~d}, J=11 \mathrm{~Hz}, 1 \mathrm{H}), 5.63(\mathrm{~d}, J=11 \mathrm{~Hz}, 1 \mathrm{H}), 5.48(\mathrm{~d}, J=10 \mathrm{~Hz}, 1 \mathrm{H}), 5.40(\mathrm{~d}, J$ $=10 \mathrm{~Hz}, 1 \mathrm{H}), 2.91(\mathrm{~s}, 3 \mathrm{H}) ;{ }^{13} \mathrm{C}\left\{{ }^{1} \mathrm{H}\right\}-\mathrm{NMR}\left(100 \mathrm{MHz}, \mathrm{CDCl}_{3}, \mathrm{TMS}\right) \delta_{\mathrm{C}} 145.3,139.6,138.8,137.7$, $136.7,132.2$, 129.5, 128.9, 128.8, 127.8, 127.7, 127.19, 127.17, 126.92, 126.88, 125.8, 125.6, 124.9, 124.4, 123.6, 123.3, 122.9, 122.4, 122.2, 120.7, 119.9, 119.1, 113.1, 111.9, 109.9, 108.4, 50.3, 50.1, 14.2; IR (neat): $v$ 3066, 3026, 1596, 1472, 1346, 1024, 738, $695 \mathrm{~cm}^{-1}$; HRMS (ESI-FTMS) m/z: $[\mathrm{M}+\mathrm{H}]^{+}$Calcd for $\mathrm{C}_{39} \mathrm{H}_{31} \mathrm{~N}_{2}$ 527.2482; found 527.2482. 

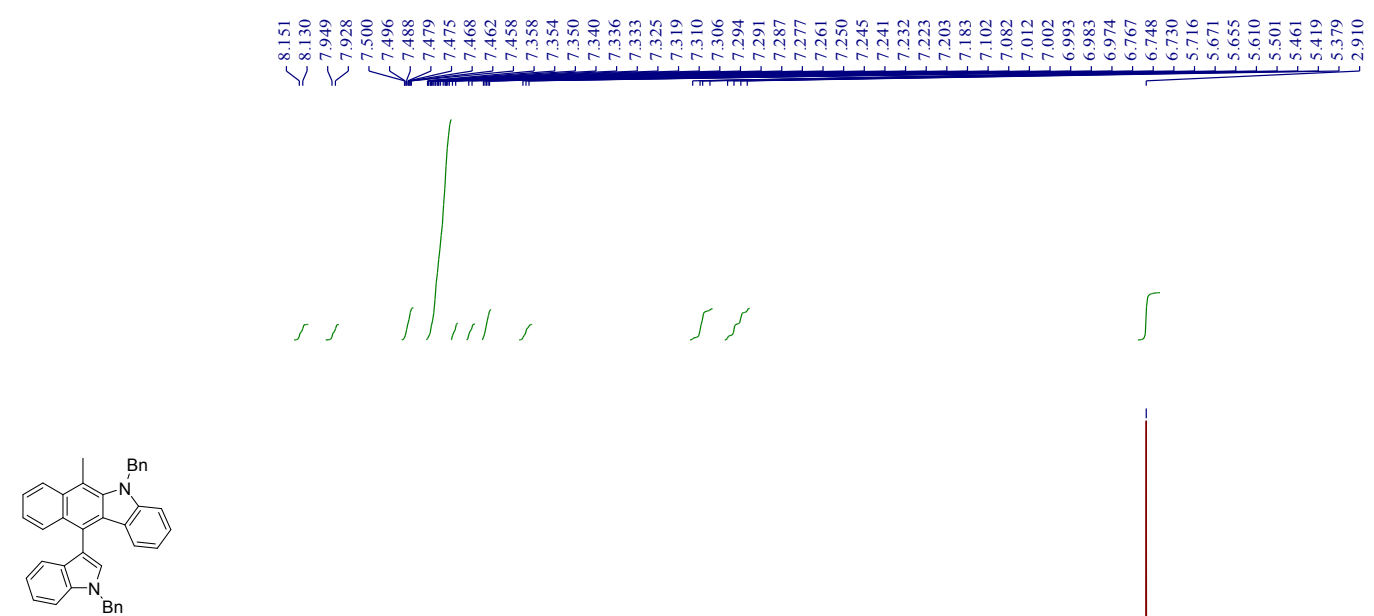

$3 m\left({ }^{1} \mathrm{H} \mathrm{NMR}, 400 \mathrm{MHz}, \mathrm{CDCl}_{3}\right)$
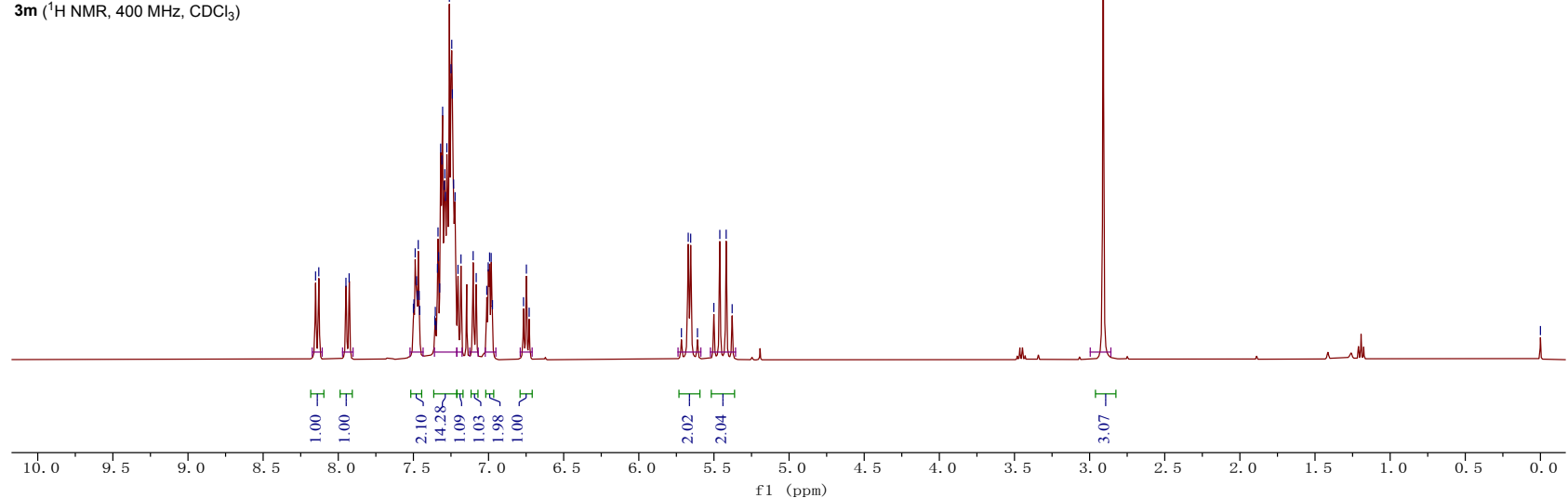

舟

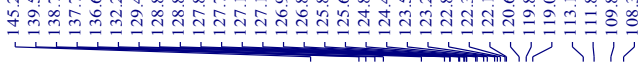

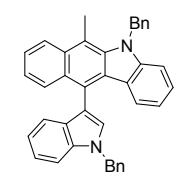

$3 \mathrm{~m}\left({ }^{13} \mathrm{C} \mathrm{NMR}, 100 \mathrm{MHz}, \mathrm{CDCl}_{3}\right)$
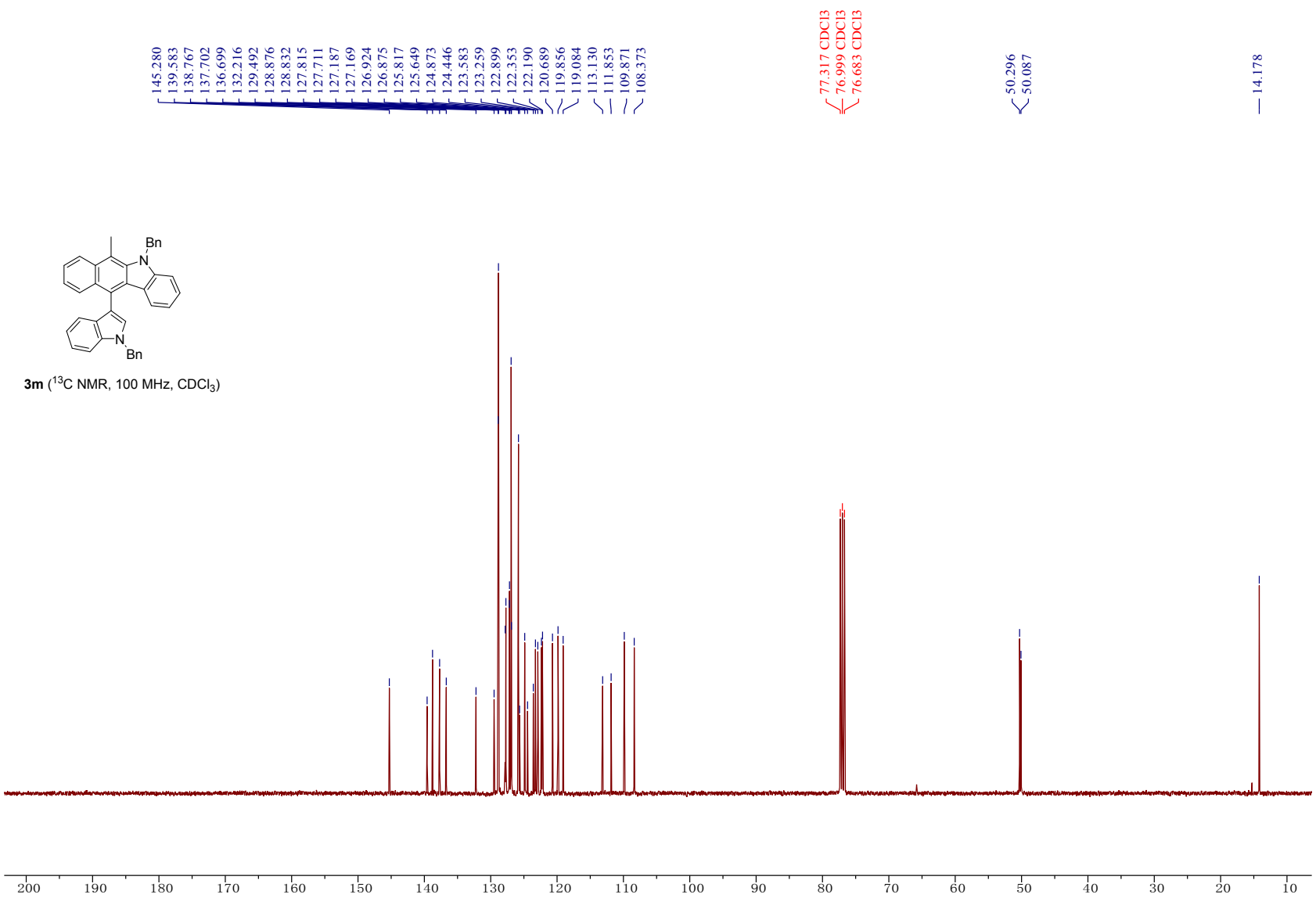

$110 \quad 100$

$80 \quad \frac{1}{10}$

60

$30+20, \frac{1}{10}$ 


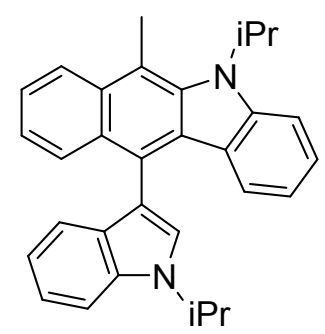

Compound 3n: Yield: $34 \mathrm{mg}, 39 \%$; a yellow solid; Mp: 92-94 ${ }^{\circ} \mathrm{C}$; Eluent: PE/EA = 5/1. ${ }^{1} \mathrm{H}$ NMR (400 MHz, $\left.\mathrm{CDCl}_{3}, \mathrm{TMS}\right) \delta 8.21(\mathrm{~d}, J=8.6 \mathrm{~Hz}, 1 \mathrm{H}), 7.88(\mathrm{~d}, J=8.6 \mathrm{~Hz}, 1 \mathrm{H}), 7.60-7.47(\mathrm{~m}, 3 \mathrm{H})$, $7.36(\mathrm{~s}, 1 \mathrm{H}), 7.32-7.23(\mathrm{~m}, 3 \mathrm{H}), 7.14(\mathrm{~d}, J=7.9 \mathrm{~Hz}, 1 \mathrm{H}), 6.98(\mathrm{t}, J=7.5 \mathrm{~Hz}, 1 \mathrm{H}), 6.90(\mathrm{~d}, J=7.9$ $\mathrm{Hz}, 1 \mathrm{H}), 6.76(\mathrm{t}, J=8.0 \mathrm{~Hz}, 1 \mathrm{H}), 5.41-5.29(\mathrm{~m}, 1 \mathrm{H}), 4.93-4.85(\mathrm{~m}, 1 \mathrm{H}), 3.10(\mathrm{~s}, 3 \mathrm{H}), 1.73(\mathrm{~d}, J=$ $7.0 \mathrm{~Hz}, 3 \mathrm{H}), 1.67-1.61(\mathrm{~m}, 9 \mathrm{H}) ;{ }^{13} \mathrm{C}\left\{{ }^{1} \mathrm{H}\right\}-\mathrm{NMR}\left(100 \mathrm{MHz}, \mathrm{CDCl}_{3}, \mathrm{TMS}\right) \delta_{\mathrm{C}} 143.8,141.7,136.0$, $132.6,129.6,128.6,127.3,126.2,125.9,125.8,124.8,124.3,123.5,123.3,123.0,122.2,121.6$, 120.7, 119.5, 118.8, 112.7, 112.6, 112.3, 109.5, 49.9, 47.2, 23.3, 22.9, 21.6, 21.2, 16.1; IR (neat): $v$ 2972, 2922, 1590, 1459, 1385, 1367, 1216, $738 \mathrm{~cm}^{-1}$; HRMS (ESI-FTMS) m/z: [M+H] $]^{+}$Calcd for $\mathrm{C}_{31} \mathrm{H}_{31} \mathrm{~N}_{2}$ 431.2482; found 431.2482 . 


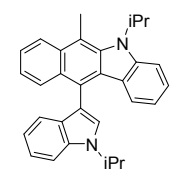

3n ( ${ }^{1} \mathrm{H} \mathrm{NMR}, 400 \mathrm{MHz}, \mathrm{CDCl}_{3}$ )
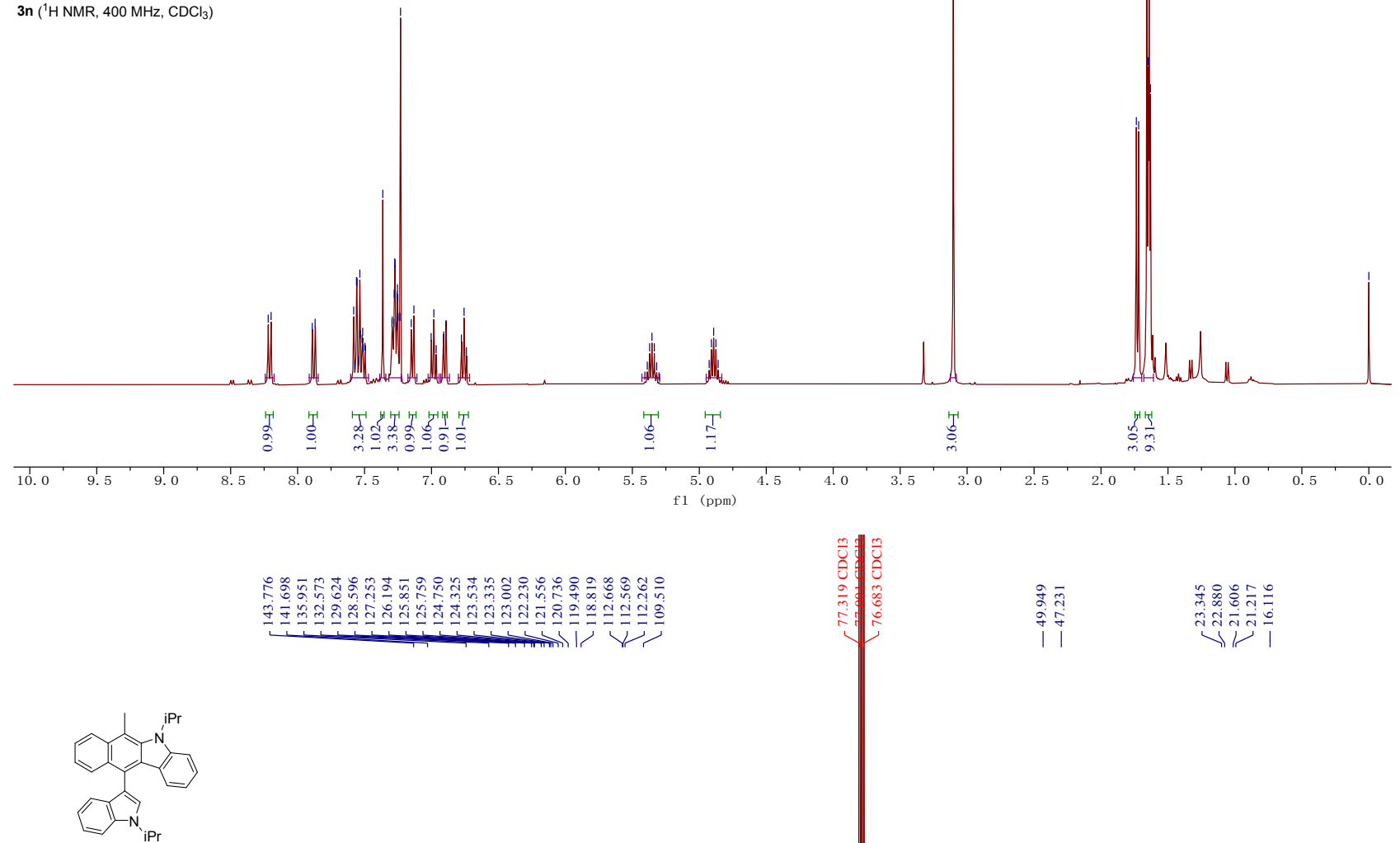

3n $\left({ }^{13} \mathrm{C} \mathrm{NMR}, 100 \mathrm{MHz}, \mathrm{CDCl}_{3}\right)$

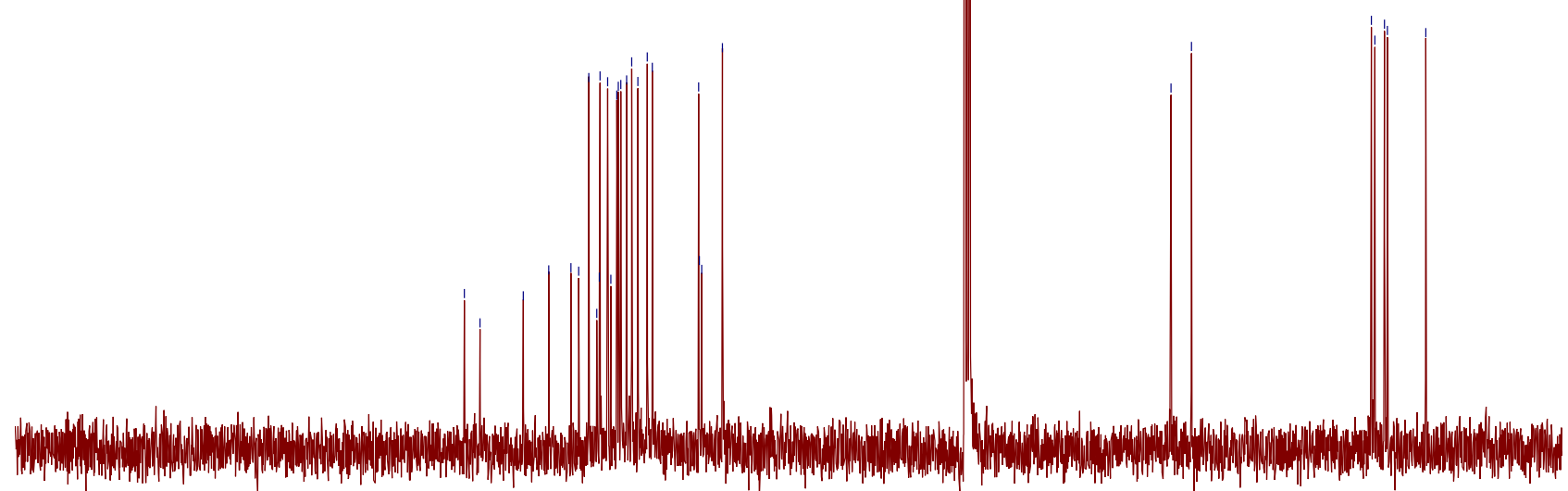

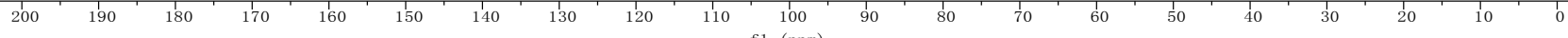




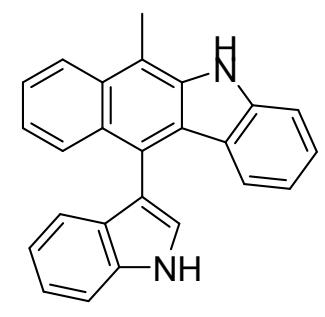

Compound 3o: Yield: $40 \mathrm{mg}$, 58\%; a yellow solid; Mp: $>200{ }^{\circ} \mathrm{C}$; Eluent: PE/DCM=1/1. ${ }^{1} \mathrm{H}$ NMR (400 MHz, $\mathrm{CD}_{2} \mathrm{Cl}_{2}$, TMS) $\delta 8.58(\mathrm{~s}, 1 \mathrm{H}), 8.11(\mathrm{~d}, J=8.6 \mathrm{~Hz}, 1 \mathrm{H}), 7.99(\mathrm{~s}, 1 \mathrm{H}), 7.82(\mathrm{~d}, J=8.6 \mathrm{~Hz}$, 1H), $7.53(\mathrm{~d}, J=8.3 \mathrm{~Hz}, 1 \mathrm{H}), 7.47-7.40(\mathrm{~m}, 1 \mathrm{H}), 7.35-7.29(\mathrm{~m}, 2 \mathrm{H}), 7.23(\mathrm{t}, J=7.7 \mathrm{~Hz}, 1 \mathrm{H})$, $7.20-7.13(\mathrm{~m}, 2 \mathrm{H}), 6.96(\mathrm{~d}, J=7.9 \mathrm{~Hz}, 1 \mathrm{H}), 6.92-6.84(\mathrm{~m}, 2 \mathrm{H}), 6.70(\mathrm{t}, J=7.5 \mathrm{~Hz}, 1 \mathrm{H}), 2.82(\mathrm{~s}$, $3 \mathrm{H}) ;{ }^{13} \mathrm{C}\left\{{ }^{1} \mathrm{H}\right\}-\mathrm{NMR}\left(100 \mathrm{MHz}, \mathrm{CD}_{2} \mathrm{Cl}_{2}\right.$, TMS $) \delta_{\mathrm{C}} 142.2,138.0,136.3,131.1,129.3,128.0,127.1$, $126.8,124.7,124.5,124.0,123.9,123.1,122.7,122.3,122.1,119.9,118.8,113.5,111.4,110.9$, 109.9, 12.6; IR (neat): v 3436, 3411, 3058, 3034, 1610, 1455, 1097, 757, $739 \mathrm{~cm}^{-1}$; HRMS (ESI-FTMS) m/z: [M+H] $]^{+}$Calcd for $\mathrm{C}_{25} \mathrm{H}_{19} \mathrm{~N}_{2}$ 347.1543; found 347.1545. 


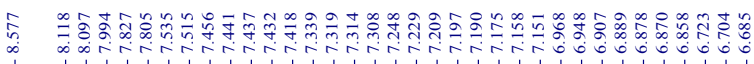

f a dithif

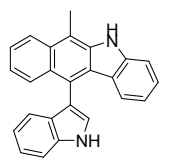

3o ( ${ }^{1} \mathrm{H} \mathrm{NMR}, 400 \mathrm{MHz}, \mathrm{CD}_{2} \mathrm{Cl}_{2}$ )

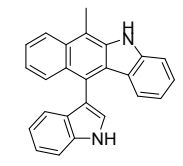

3o $\left({ }^{13} \mathrm{C} \mathrm{NMR}, 100 \mathrm{MHz}, \mathrm{CD}_{2} \mathrm{Cl}_{2}\right)$

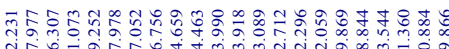

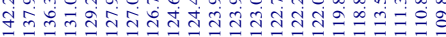
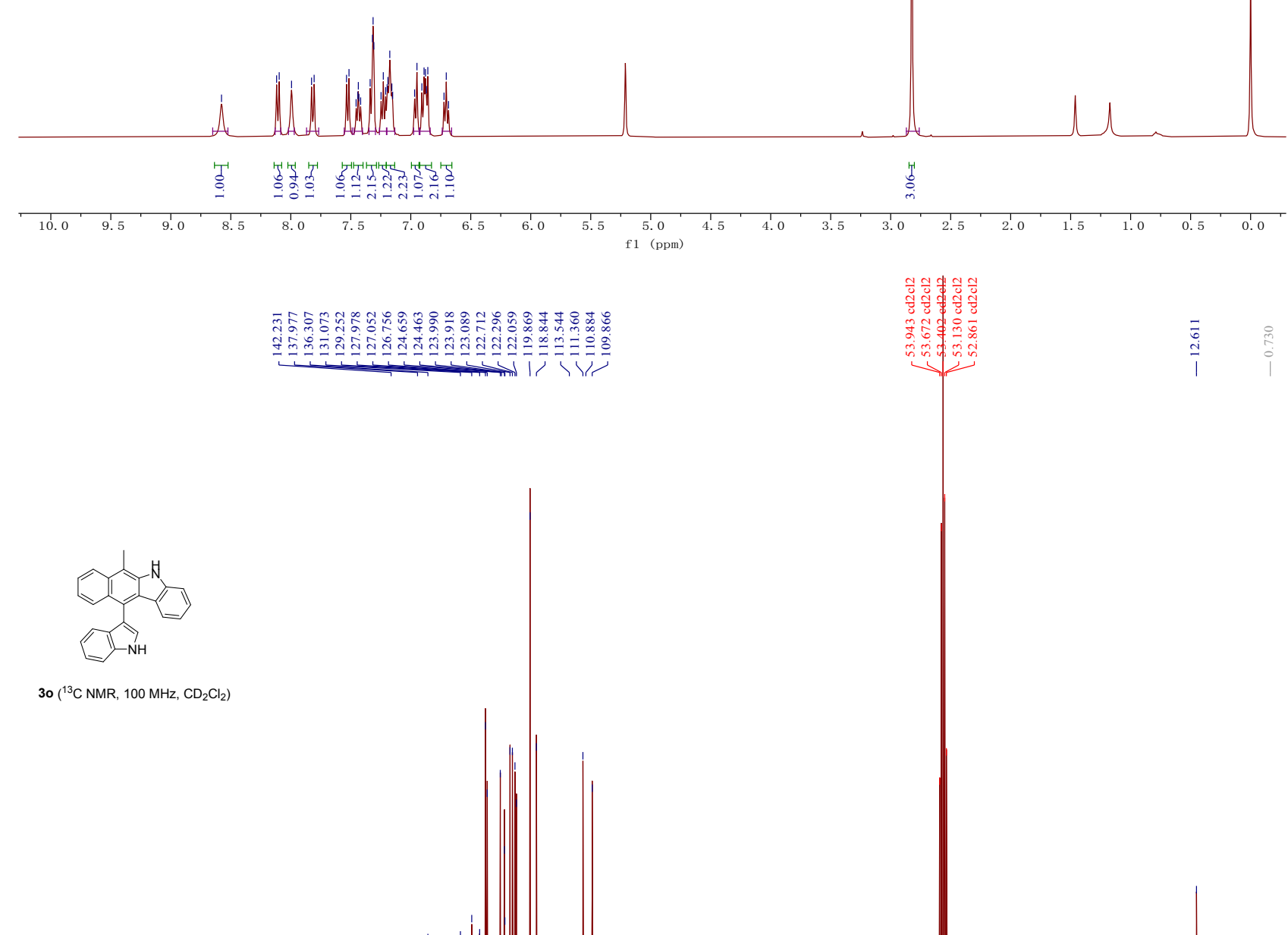

I.
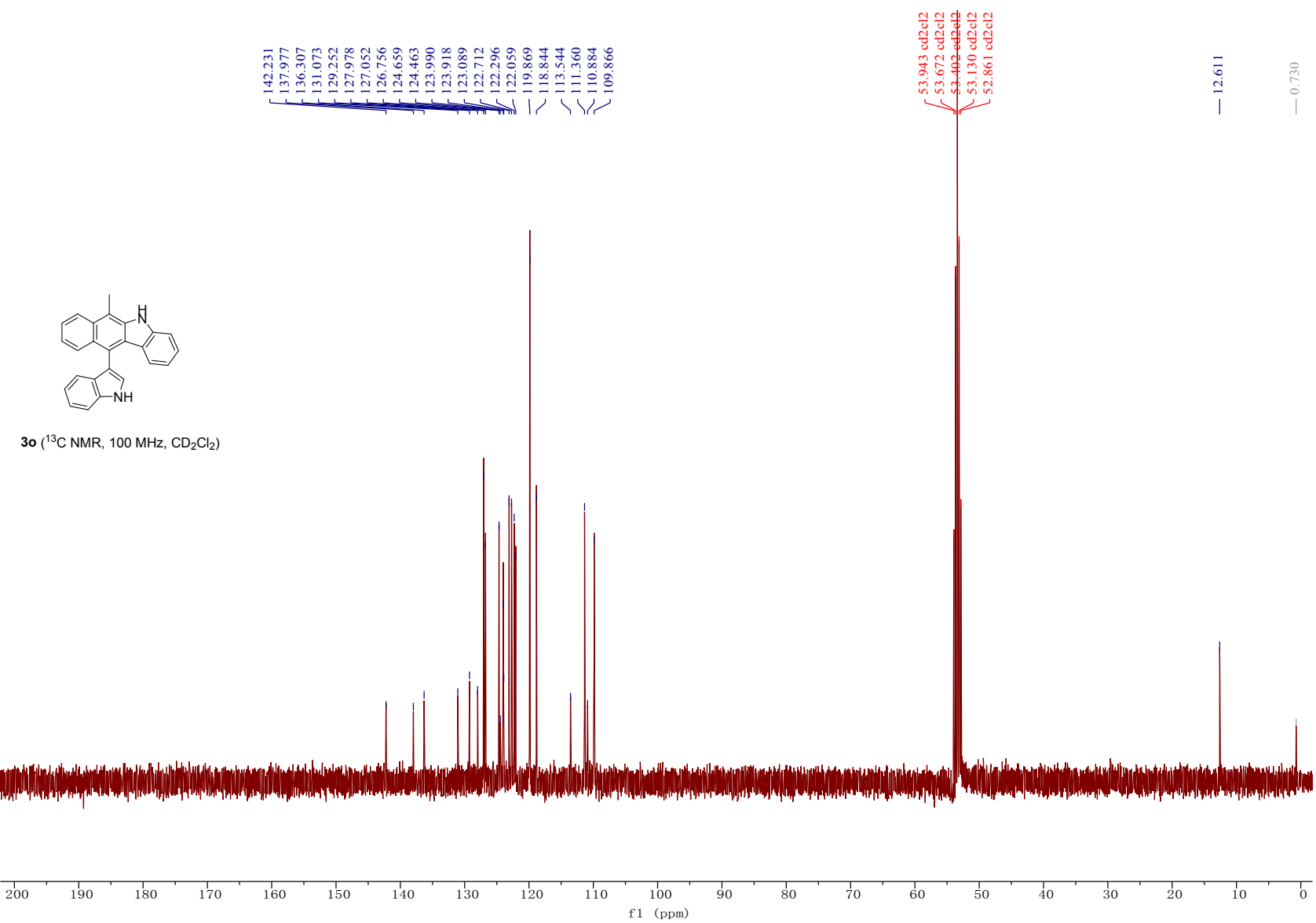


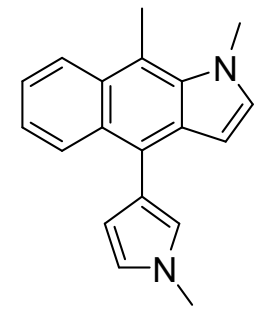

Compound 3p: Yield: 9 mg, 16\%; green oil; Eluent: $\mathrm{PE} / \mathrm{DCM}=3 / 1 .{ }^{1} \mathrm{H}$ NMR $\left(400 \mathrm{MHz}, \mathrm{CDCl}_{3}\right.$, TMS) $\delta 8.15(\mathrm{~d}, J=8.5 \mathrm{~Hz}, 1 \mathrm{H}), 7.61(\mathrm{~d}, J=8.5 \mathrm{~Hz}, 1 \mathrm{H}), 7.38(\mathrm{t}, J=7.3 \mathrm{~Hz}, 1 \mathrm{H}), 7.31(\mathrm{t}, J=7.4$ $\mathrm{Hz}, 1 \mathrm{H}), 7.15(\mathrm{~d}, J=3.4 \mathrm{~Hz}, 1 \mathrm{H}), 6.87$ (t, $J=2.3 \mathrm{~Hz}, 1 \mathrm{H}), 6.70(\mathrm{~d}, J=3.4 \mathrm{~Hz}, 1 \mathrm{H}), 6.34$ (t, $J=3.2$ $\mathrm{Hz}, 1 \mathrm{H}), 6.28(\mathrm{~d}, J=3.7 \mathrm{~Hz}, 1 \mathrm{H}), 3.25(\mathrm{~s}, 3 \mathrm{H}), 3.24(\mathrm{~s}, 3 \mathrm{H}), 2.96(\mathrm{~s}, 3 \mathrm{H}) ;{ }^{13} \mathrm{C}\left\{{ }^{1} \mathrm{H}\right\}-\mathrm{NMR}(100 \mathrm{MHz}$, $\left.\mathrm{CDCl}_{3}, \mathrm{TMS}\right) \delta_{\mathrm{C}} 136.0,134.1,131.6,130.2,127.8,126.4,125.6,125.5,123.9,123.7,122.2,121.2$, 112.3, 108.1, 107.6, 99.0, 34.6, 34.0, 15.1; IR (neat): $v$ 2917, 1527, 1383, 1315, 1084, 753, 738, 705 $\mathrm{cm}^{-1}$; HRMS (ESI-FTMS) m/z: [M+H] $]^{+}$Calcd for $\mathrm{C}_{19} \mathrm{H}_{19} \mathrm{~N}_{2} 275.1543$; found 275.1543. 


\section{| | | | | || ||}

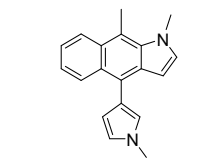

3p ( ${ }^{1} \mathrm{H} \mathrm{NMR}, 400 \mathrm{MHz}, \mathrm{CDCl}_{3}$ )

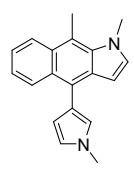

$3 p\left({ }^{13} \mathrm{C} \mathrm{NMR}, 100 \mathrm{MHz}, \mathrm{CDCl}_{3}\right)$ 


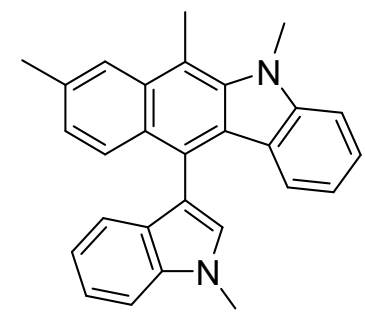

Compound 4a: Yield: $72 \mathrm{mg}$, 93\%; a yellow solid; Mp: 106-108 ${ }^{\circ} \mathrm{C}$; Eluent: $\mathrm{PE} / \mathrm{DCM}=3 / 1 .{ }^{1} \mathrm{H}$ NMR (400 MHz, $\mathrm{CDCl}_{3}$, TMS) $\delta 7.98$ (s, 1H), $7.80(\mathrm{~d}, J=8.6 \mathrm{~Hz}, 1 \mathrm{H}), 7.52(\mathrm{~d}, J=8.6 \mathrm{~Hz}, 1 \mathrm{H})$, $7.38-7.29$ (m, 2H), 7.27 (d, $J=8.1 \mathrm{~Hz}, 1 \mathrm{H}), 7.17$ (s, 1H), $7.14-7.05$ (m, 2H), 6.98 (t, $J=7.5 \mathrm{~Hz}$, 1H), 6.91 (d, $J=7.8 \mathrm{~Hz}, 1 \mathrm{H}), 6.76(\mathrm{t}, J=7.5 \mathrm{~Hz}, 1 \mathrm{H}), 4.13$ (s, 3H), 3.98 (s, 3H), 3.17 (s, 3H), 2.58 (s, $3 \mathrm{H}) ;{ }^{13} \mathrm{C}\left\{{ }^{1} \mathrm{H}\right\}$-NMR (100 MHz, CDCl 3 , TMS) $\delta_{\mathrm{C}} 145.5,140.3,137.1,134.3,132.4,128.6,128.3$, 127.8, 127.1, 126.4, 124.8, 124.5, 124.2, 123.6, 123.0, 121.8, 120.6, 119.5, 118.6, 112.5, 111.0, 109.3, 107.9, 34.6, 33.1, 22.2, 14.7; IR (neat): v 3058, 2914, 1595, 1471, 1117, 815, 784, $737 \mathrm{~cm}^{-1}$; HRMS (EI-TOF) m/z: [M] $]^{+}$Calcd for $\mathrm{C}_{28} \mathrm{H}_{24} \mathrm{~N}_{2}$ 388.1934; found 388.1939 . 


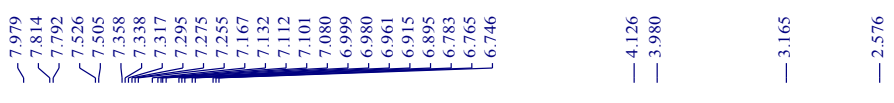

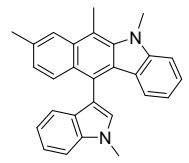

4a ( ${ }^{1} \mathrm{H} \mathrm{NMR}, 400 \mathrm{MHz}, \mathrm{CDCl}_{3}$ )
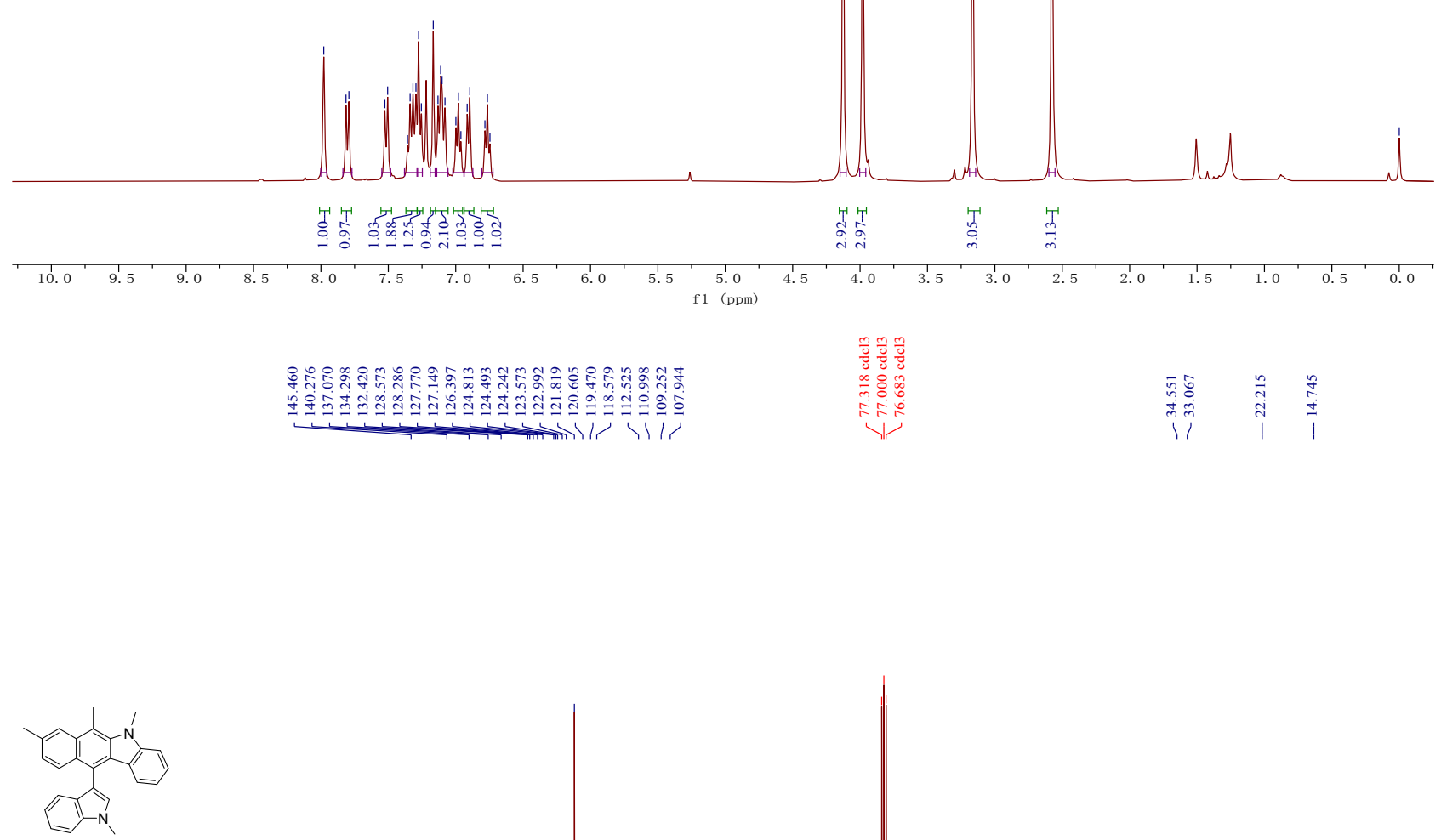

4a $\left({ }^{13} \mathrm{C} \mathrm{NMR}, 100 \mathrm{MHz}, \mathrm{CDCl}_{3}\right)$
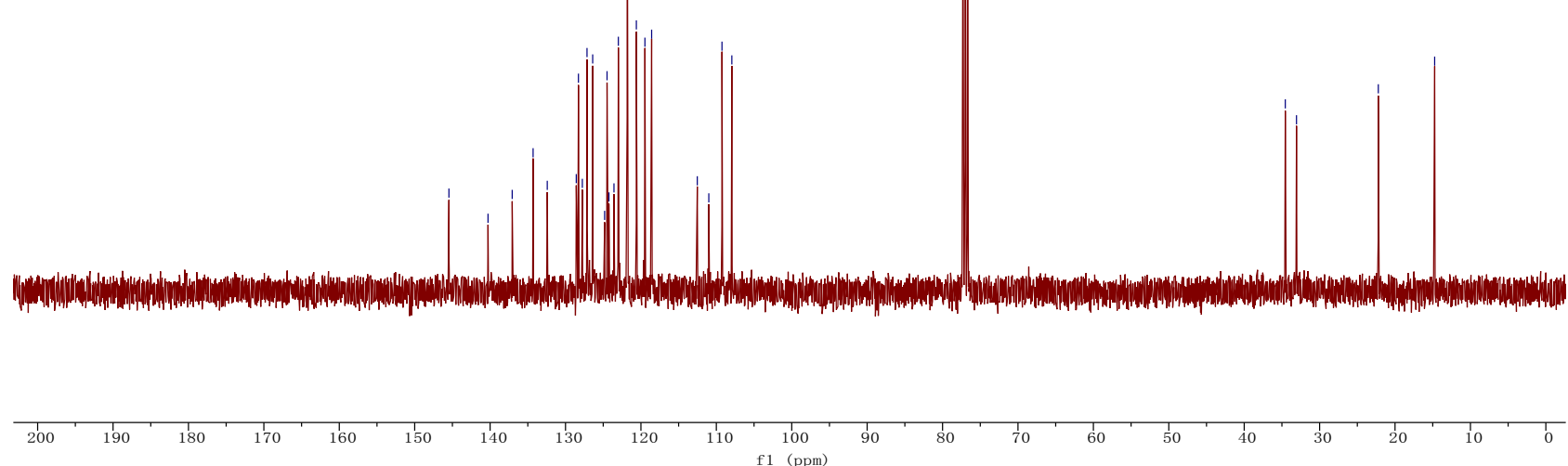


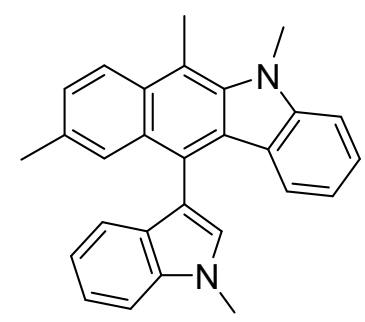

Compound 4b: Yield: $62 \mathrm{mg}$, 80\%; a yellow solid; Mp: 136-138 ${ }^{\circ} \mathrm{C}$; Eluent: $\mathrm{PE} / \mathrm{DCM}=3 / 1 .{ }^{1} \mathrm{H}$ NMR (400 MHz, $\left.\mathrm{CDCl}_{3}, \mathrm{TMS}\right) \delta 8.13(\mathrm{~d}, J=8.8 \mathrm{~Hz}, 1 \mathrm{H}), 7.68(\mathrm{~s}, 1 \mathrm{H}), 7.53(\mathrm{~d}, J=8.4 \mathrm{~Hz}, 1 \mathrm{H})$, $7.39-7.26(\mathrm{~m}, 4 \mathrm{H}), 7.22(\mathrm{~s}, 1 \mathrm{H}), 7.16(\mathrm{~s}, 1 \mathrm{H}), 7.12(\mathrm{~d}, J=7.8 \mathrm{~Hz}, 1 \mathrm{H}), 6.98(\mathrm{t}, J=7.5 \mathrm{~Hz}, 1 \mathrm{H})$, $6.81(\mathrm{~d}, J=7.8 \mathrm{~Hz}, 1 \mathrm{H}), 6.75(\mathrm{t}, J=7.5 \mathrm{~Hz}, 1 \mathrm{H}), 4.12(\mathrm{~s}, 3 \mathrm{H}), 3.99$ (s, 3H), $3.18(\mathrm{~s}, 3 \mathrm{H}), 2.37$ (s, $3 \mathrm{H}) ;{ }^{13} \mathrm{C}\left\{{ }^{1} \mathrm{H}\right\}-\mathrm{NMR}\left(100 \mathrm{MHz}, \mathrm{CDCl}_{3}\right.$, TMS $) \delta_{\mathrm{C}} 145.5,139.5,137.1,131.4,130.5,129.7,128.6$, $128.2,127.2,126.5,125.8,125.6,123.6,123.5,123.1,122.7,121.8,120.6,119.4,118.5,112.5$, 111.7, 109.2, 107.9, 34.5, 33.1, 21.5, 14.7; IR (neat): v 2922, 2854, 1475, 1370, 1345, 1320, 806, $738 \mathrm{~cm}^{-1}$; HRMS (EI-TOF) m/z: Calcd for $\mathrm{C}_{28} \mathrm{H}_{24} \mathrm{~N}_{2}$ [M] $]^{+}: 388.1934$; found 388.1940. 

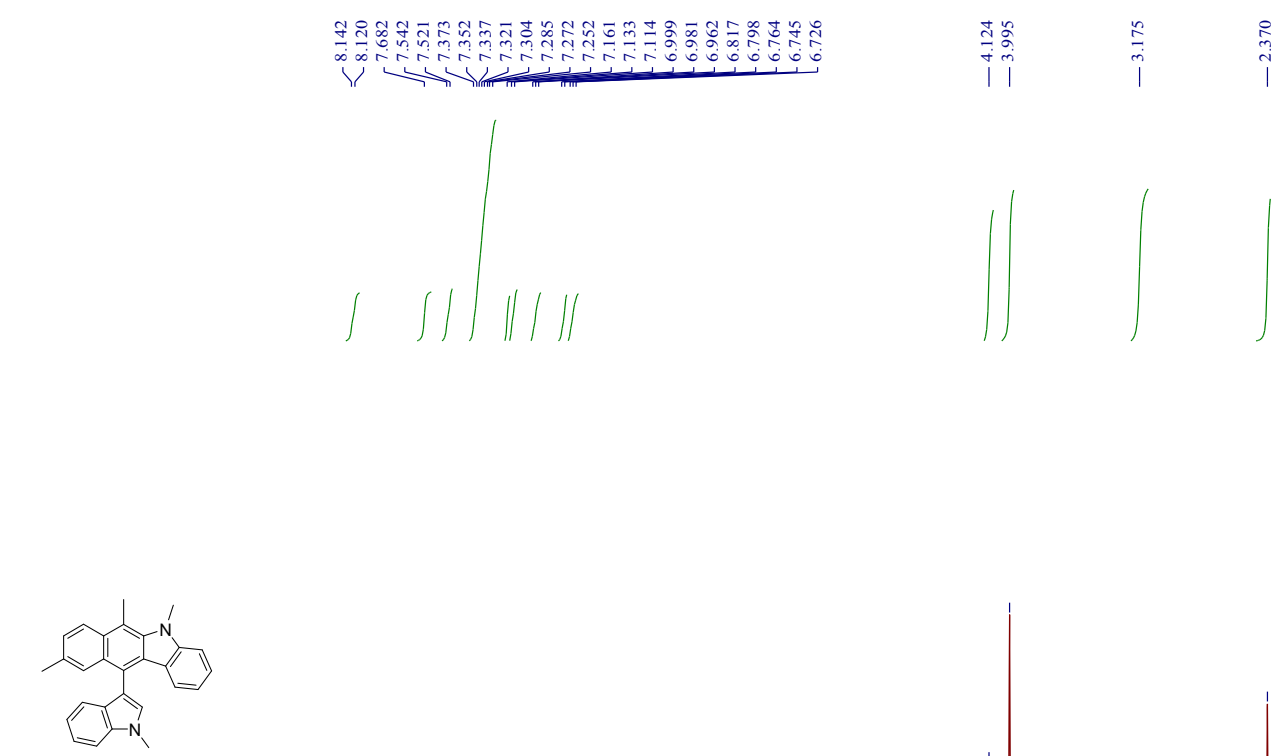

4b ( ${ }^{1} \mathrm{H} \mathrm{NMR}, 400 \mathrm{MHz}, \mathrm{CDCl}_{3}$ )
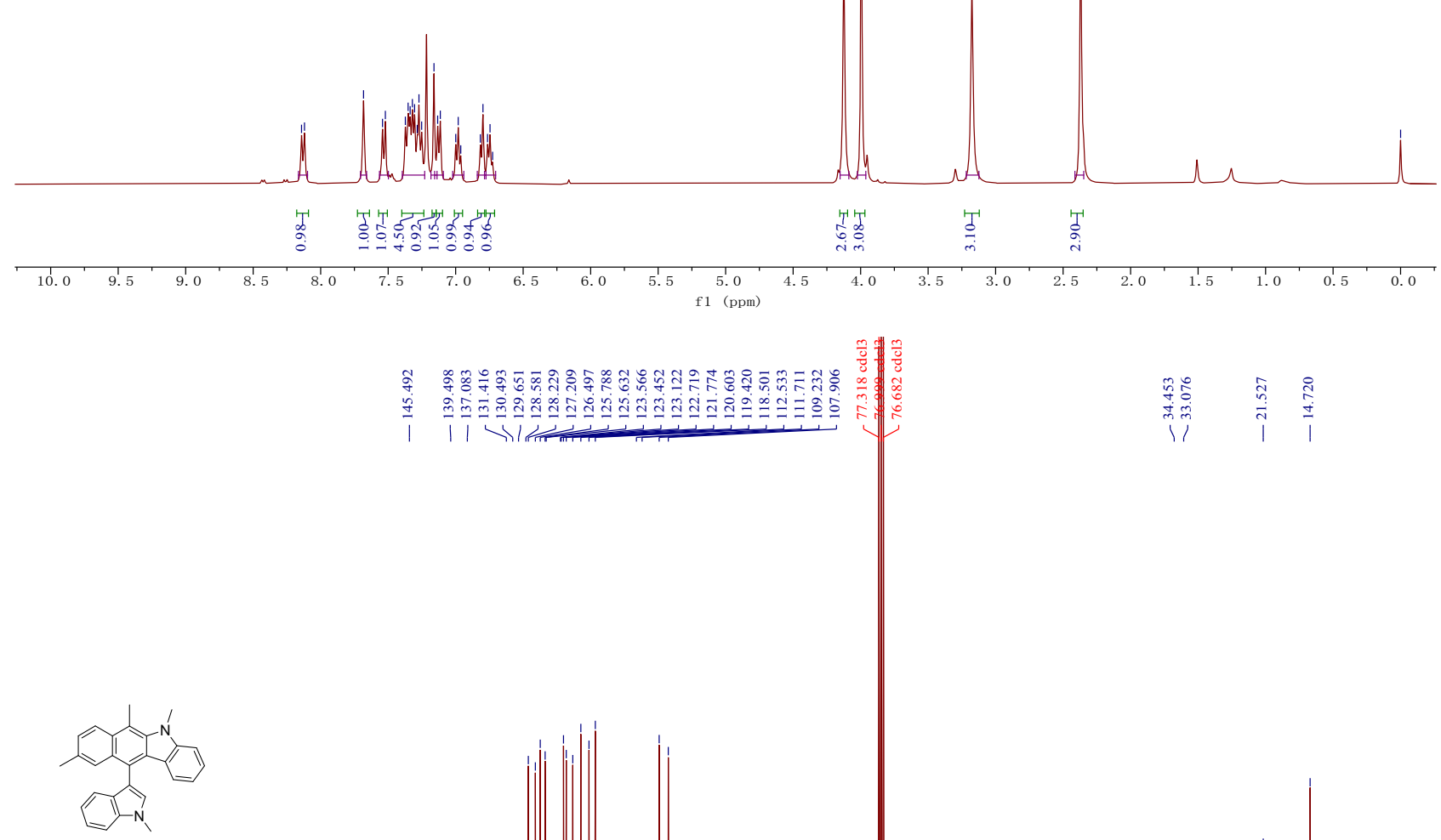

4b $\left({ }^{13} \mathrm{C} \mathrm{NMR}, 100 \mathrm{MHz}, \mathrm{CDCl}_{3}\right)$
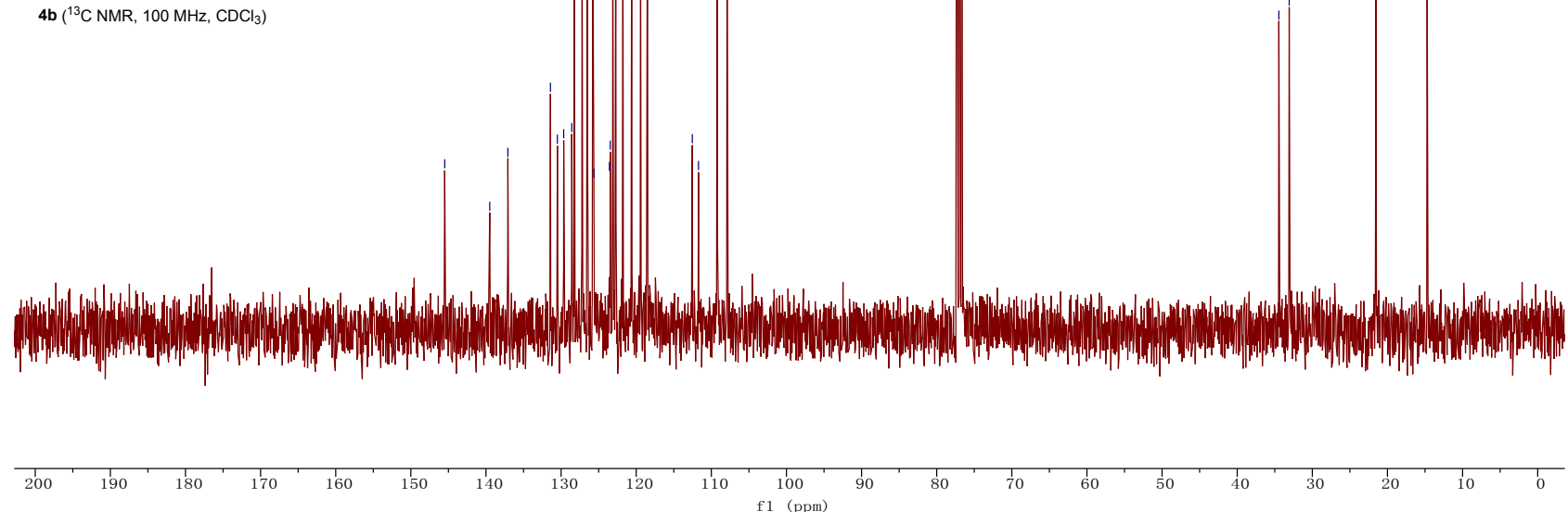


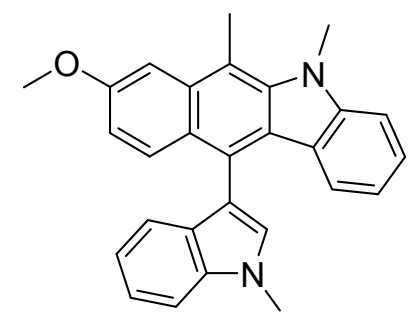

Compound 4c: Yield: 65 mg, 80\%; a yellow solid; Mp: $>200{ }^{\circ} \mathrm{C}$; Eluent: PE/DCM =2/1. ${ }^{1} \mathrm{H}$ NMR $\left(400 \mathrm{MHz}, \mathrm{CDCl}_{3}, \mathrm{TMS}\right) \delta 7.82(\mathrm{~d}, J=9.3 \mathrm{~Hz}, 1 \mathrm{H}), 7.51(\mathrm{~d}, J=8.2 \mathrm{~Hz}, 1 \mathrm{H}), 7.43$ (s, 1H), 7.37 $7.25(\mathrm{~m} \mathrm{3H}), 7.20-7.08(\mathrm{~m}, 2 \mathrm{H}), 7.04-6.84(\mathrm{~m}, 3 \mathrm{H}), 6.78(\mathrm{~s}, 1 \mathrm{H}), 4.13(\mathrm{~s}, 3 \mathrm{H}), 3.98(\mathrm{~s}, 6 \mathrm{H}), 3.13$ $(\mathrm{s}, 3 \mathrm{H}) ;{ }^{13} \mathrm{C}\left\{{ }^{1} \mathrm{H}\right\}-\mathrm{NMR}\left(100 \mathrm{MHz}, \mathrm{CDCl}_{3}, \mathrm{TMS}\right) \delta_{\mathrm{C}} 157.2,145.2,140.8,137.1,133.4,129.0,128.5$, $128.3,126.1,125.1,124.5,123.7,122.7,121.9,120.6,119.5,118.7,114.7,112.5,110.3,109.3$, 107.9, 101.4, 55.3, 34.6, 33.1, 14.9; IR (neat): v 3123, 2979, 1614, 1479, 1223, 1212, 831, $746 \mathrm{~cm}^{-1}$; HRMS (EI-TOF) m/z: [M+H] $]^{+}$Calcd for $\mathrm{C}_{28} \mathrm{H}_{24} \mathrm{~N}_{2} \mathrm{O}$ 404.1883; found 404.1882. 

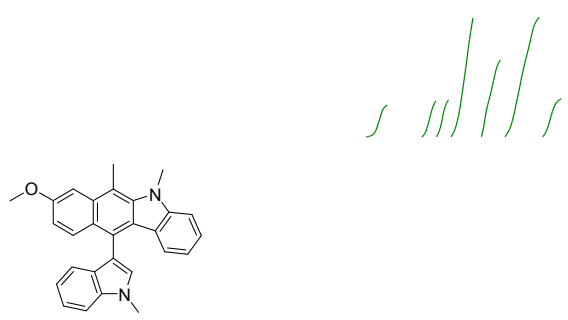

4c ( $\left.{ }^{1} \mathrm{H} \mathrm{NMR}, 400 \mathrm{MHz}, \mathrm{CDCl}_{3}\right)$
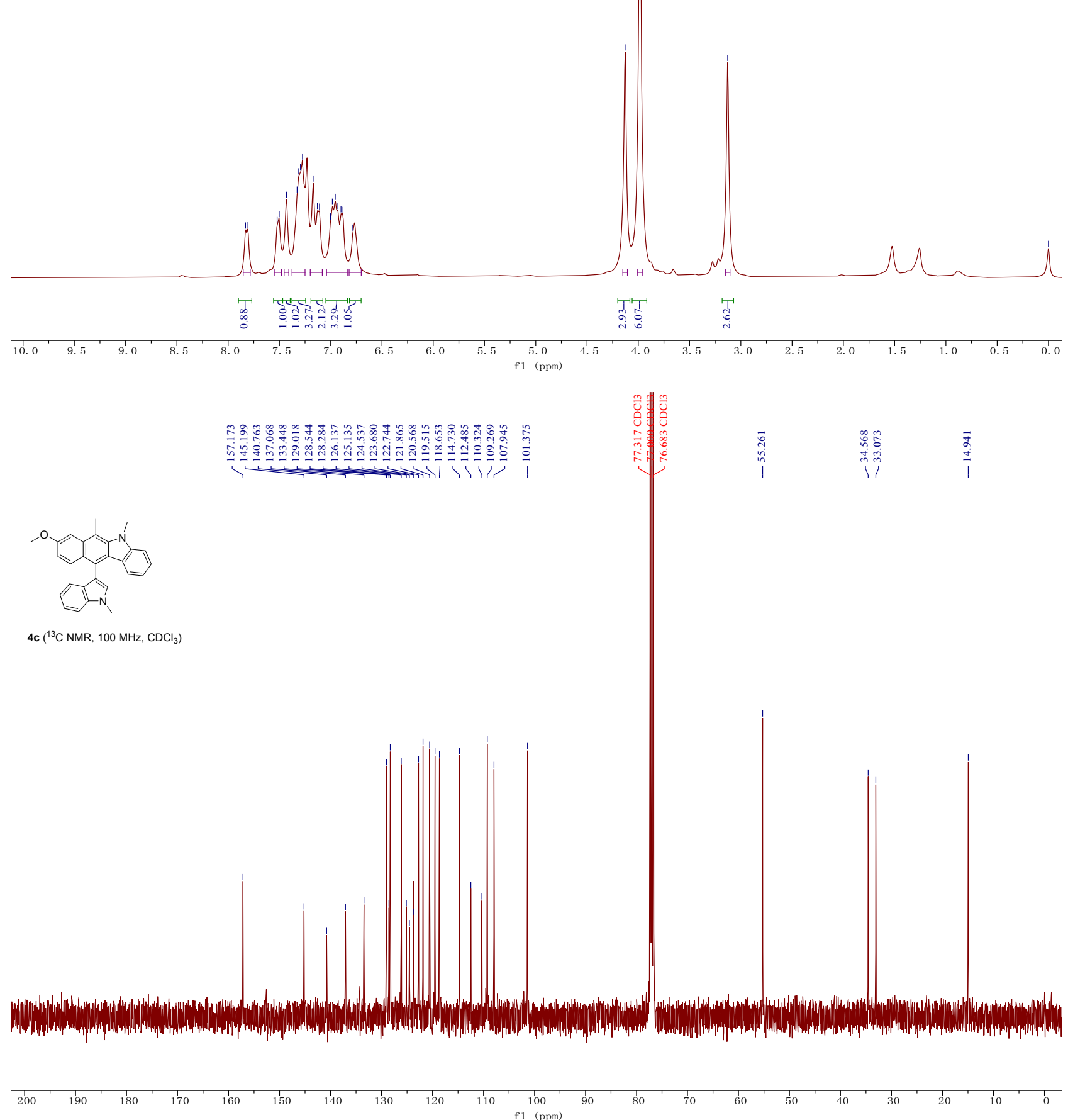


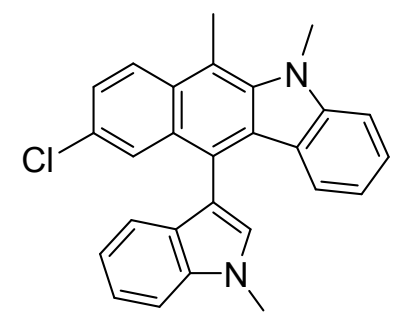

Compound 4d: Yield: $33 \mathrm{mg}, 40 \%$; a yellow solid; Mp: $>200{ }^{\circ} \mathrm{C}$; Eluent: $\mathrm{PE} / \mathrm{DCM}=1 / 1 .{ }^{1} \mathrm{H}$ NMR (400 MHz, $\left.\mathrm{CDCl}_{3}, \mathrm{TMS}\right) \delta 8.12(\mathrm{~d}, J=9.2 \mathrm{~Hz}, 1 \mathrm{H}), 7.88(\mathrm{~d}, J=2.2 \mathrm{~Hz}, 1 \mathrm{H}), 7.51(\mathrm{~d}, J=8.3$ $\mathrm{Hz}, 1 \mathrm{H}), 7.42(\mathrm{dd}, J=9.2,2.2 \mathrm{~Hz}, 1 \mathrm{H}), 7.37-7.23(\mathrm{~m}, 3 \mathrm{H}), 7.12(\mathrm{~s}, 1 \mathrm{H}), 7.07$ (d, J= $7.9 \mathrm{~Hz}, 1 \mathrm{H})$, $6.98(\mathrm{t}, J=7.4 \mathrm{~Hz}, 1 \mathrm{H}), 6.83(\mathrm{~d}, J=7.8 \mathrm{~Hz}, 1 \mathrm{H}), 6.76(\mathrm{t}, J=7.4 \mathrm{~Hz}, 1 \mathrm{H}), 4.09(\mathrm{~s}, 3 \mathrm{H}), 3.97(\mathrm{~s}, 3 \mathrm{H})$, $3.13(\mathrm{~s}, 3 \mathrm{H}) ;{ }^{13} \mathrm{C}\left\{{ }^{1} \mathrm{H}\right\}-\mathrm{NMR}\left(100 \mathrm{MHz}, \mathrm{CDCl}_{3}\right.$, TMS) $\delta_{\mathrm{C}} 145.5,140.0,137.1,130.4,130.2,128.4$, $128.3,128.1,127.0,126.4,125.6,125.4,124.6,123.7,123.4,123.0,122.0,120.3,119.7,118.9$, 112.0, 111.7, 109.4, 108.1, 34.4, 33.1, 14.8; IR (neat): v 3055, 2917, 1590, 1479, 1370, 1119, 905, $728 \mathrm{~cm}^{-1}$; HRMS (ESI-FTMS) m/z: [M+H] $]^{+}$Calcd for $\mathrm{C}_{27} \mathrm{H}_{22} \mathrm{~N}_{2} \mathrm{Cl}$ 409.1466; found 409.1464. 
$V^{\infty}$
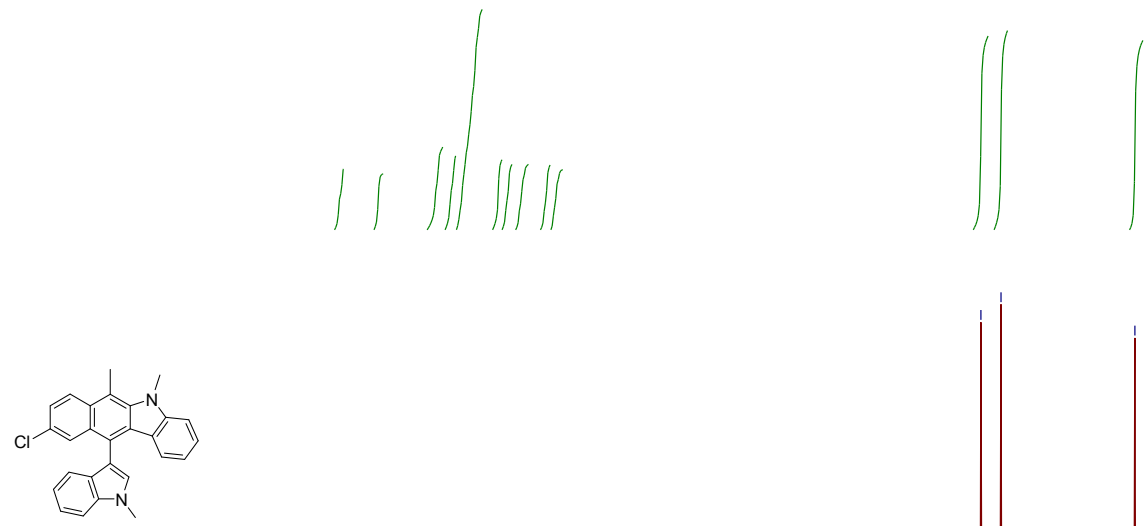

4d ( $\left.{ }^{1} \mathrm{H} \mathrm{NMR}, 400 \mathrm{MHz}, \mathrm{CDCl}_{3}\right)$
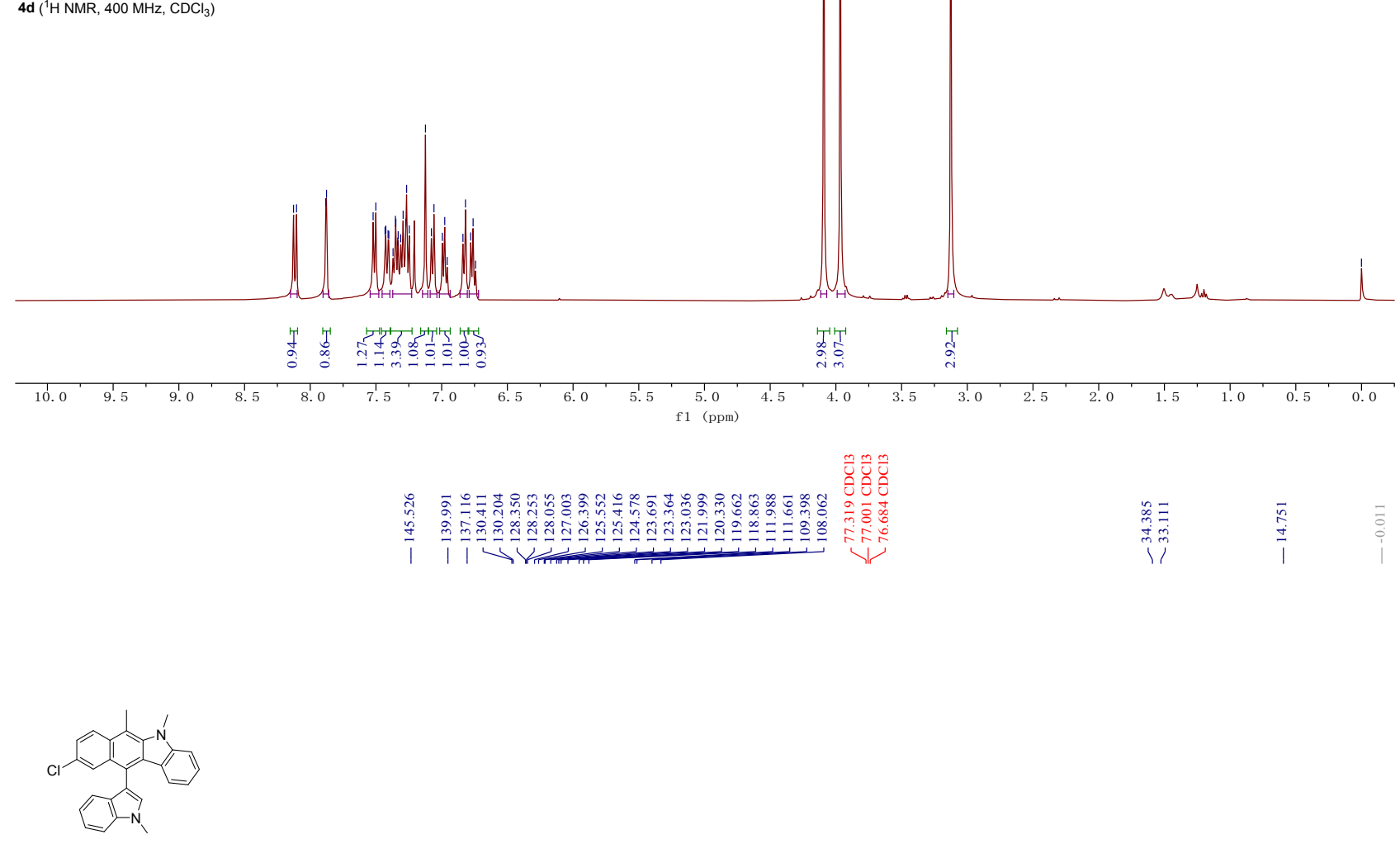

4d $\left({ }^{13} \mathrm{C} \mathrm{NMR}, 100 \mathrm{MHz}, \mathrm{CDCl}_{3}\right)$

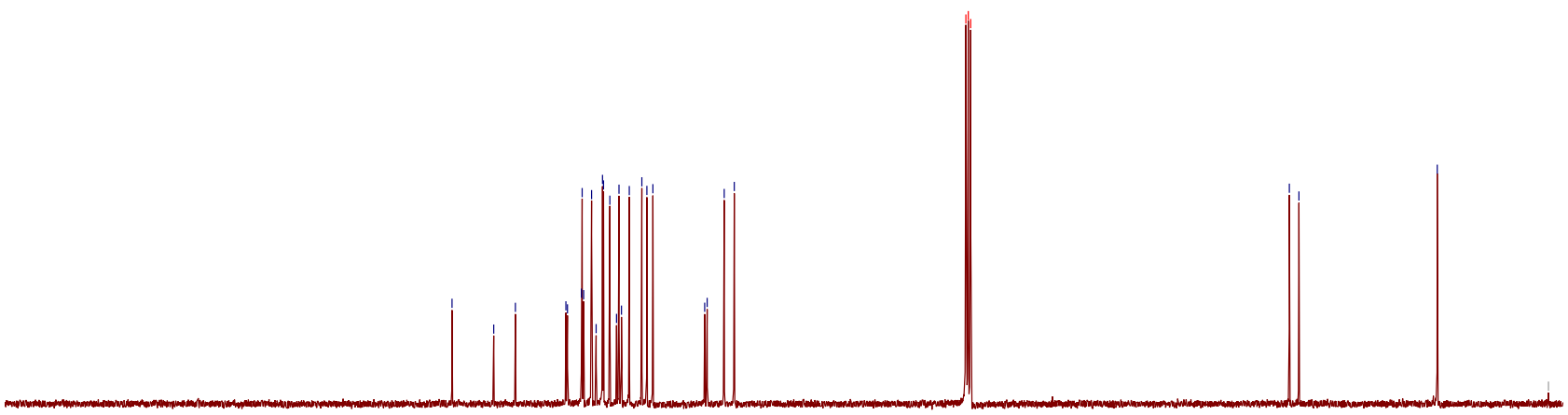

140

$130 \quad 120$

$110 \quad 100$

$90 \quad 80$

50

$20,10,1$ 


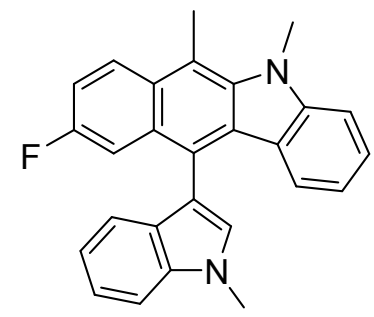

Compound 4e: Yield: $46 \mathrm{mg}$, 70\%; a yellow solid; Mp: $>200{ }^{\circ} \mathrm{C}$; Eluent: PE/DCM =2/1. ${ }^{1} \mathrm{H}$ NMR (400 MHz, $\left.\mathrm{CDCl}_{3}, \mathrm{TMS}\right) \delta 8.20(\mathrm{dd}, J=9.7,5.6 \mathrm{~Hz}, 1 \mathrm{H}), 7.53(\mathrm{~d}, J=7.1 \mathrm{~Hz}, 2 \mathrm{H}), 7.42-7.26$ (m, 4H), $7.18(\mathrm{~s}, 1 \mathrm{H}), 7.10(\mathrm{~d}, J=8.0 \mathrm{~Hz}, 1 \mathrm{H}), 7.00$ (d, $J=7.6 \mathrm{~Hz}, 1 \mathrm{H}), 6.91(\mathrm{~d}, J=7.9 \mathrm{~Hz}, 1 \mathrm{H}), 6.79$ $(\mathrm{d}, J=7.8 \mathrm{~Hz}, 1 \mathrm{H}), 4.14$ (s, 3H), 4.00 (s, 3H), 3.20 (s, 3H); ${ }^{13} \mathrm{C}\left\{{ }^{1} \mathrm{H}\right\}-\mathrm{NMR}\left(100 \mathrm{MHz}, \mathrm{CDCl}_{3}, \mathrm{TMS}\right)$ $\delta_{\mathrm{C}} 158.7(\mathrm{~d}, J=241.2 \mathrm{~Hz}), 145.6,139.6,137.1,130.2(\mathrm{~d}, J=8.0 \mathrm{~Hz}), 129.2,128.3,128.2,127.0$, 126.5, $125.1(\mathrm{~d}, J=8.5 \mathrm{~Hz}), 123.7$ (d, $J=5.8 \mathrm{~Hz}), 123.4,123.0,122.0,120.4,119.6,118.7,115.0$ $(\mathrm{d}, J=25.6 \mathrm{~Hz}), 112.2,112.0,109.8(\mathrm{~d}, J=21.4 \mathrm{~Hz}), 109.4,108.1,34.5,33.1,14.9 ;{ }^{19} \mathrm{~F}$ NMR $(376$ $\mathrm{MHz}, \mathrm{CDCl}_{3}$ ) $\delta$-119.92; IR (neat): v 2920, 2848, 1597, 1470, 1369, 1238, 967, $740 \mathrm{~cm}^{-1}$; HRMS (ESI-FTMS) m/z: [M+H] $]^{+}$Calcd for $\mathrm{C}_{27} \mathrm{H}_{22} \mathrm{~N}_{2} \mathrm{~F}$ 393.1762; found 393.1762. 


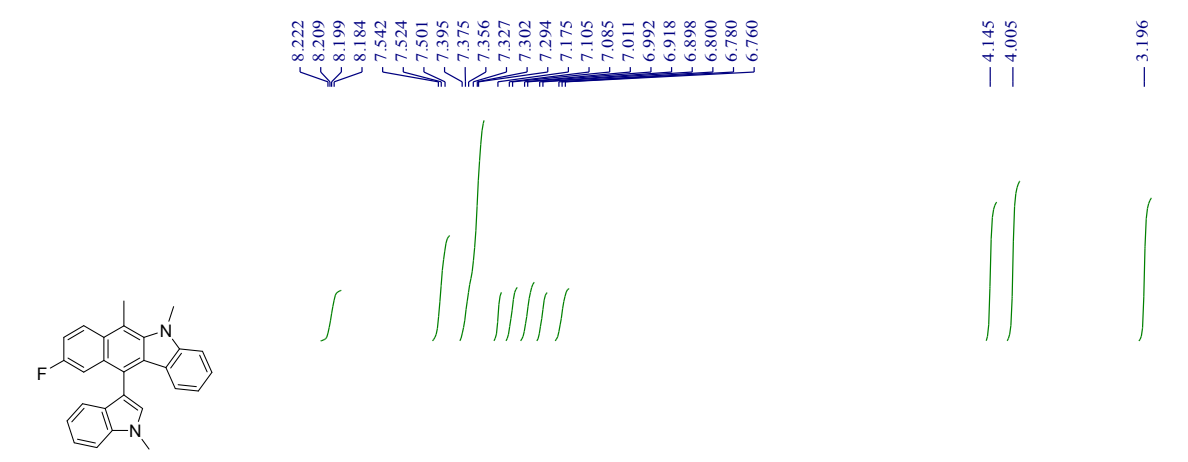

4e ( $\left.{ }^{1} \mathrm{H} \mathrm{NMR}, 400 \mathrm{MHz}, \mathrm{CDCl}_{3}\right)$
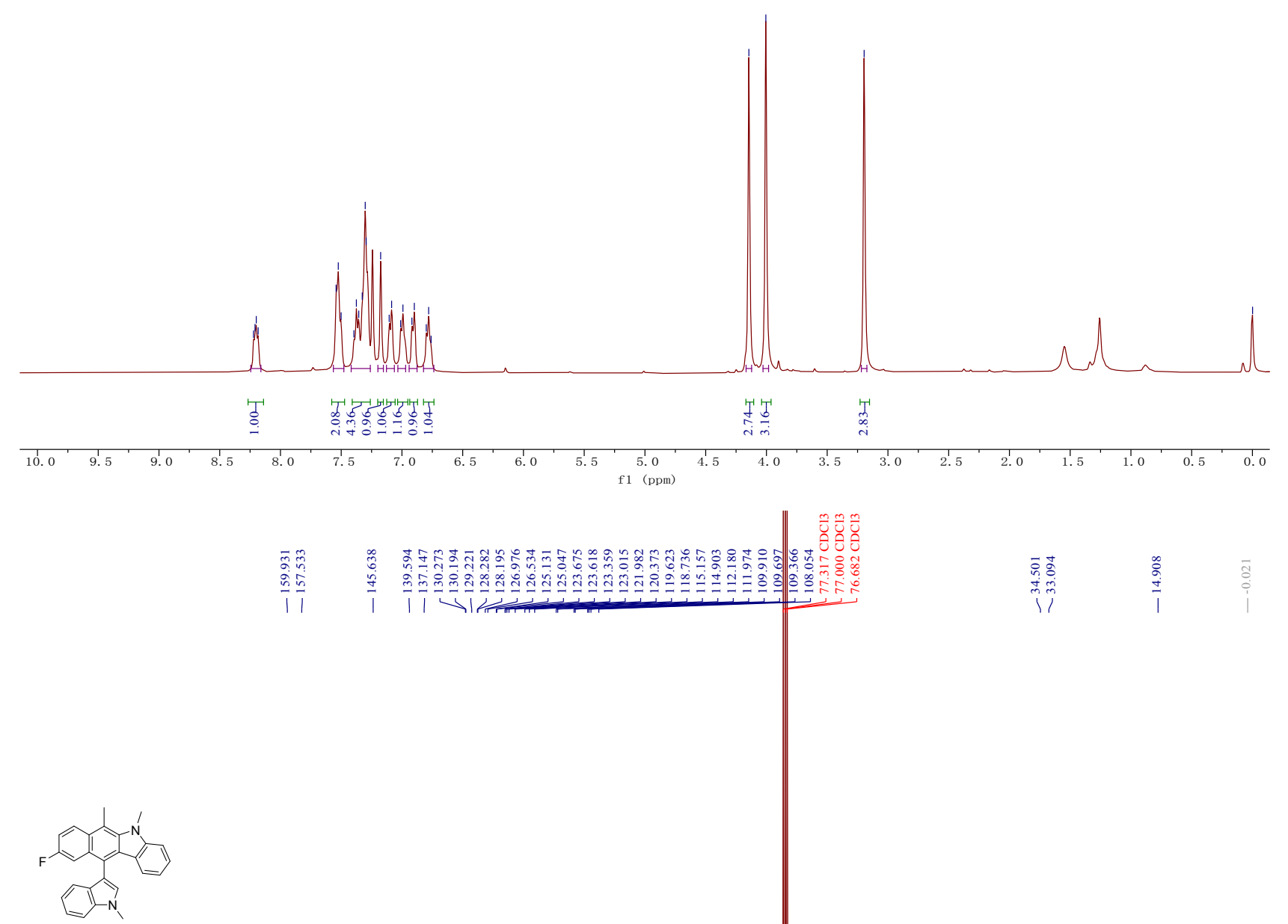

4e $\left({ }^{13} \mathrm{C} \mathrm{NMR}, 100 \mathrm{MHz}, \mathrm{CDCl}_{3}\right)$

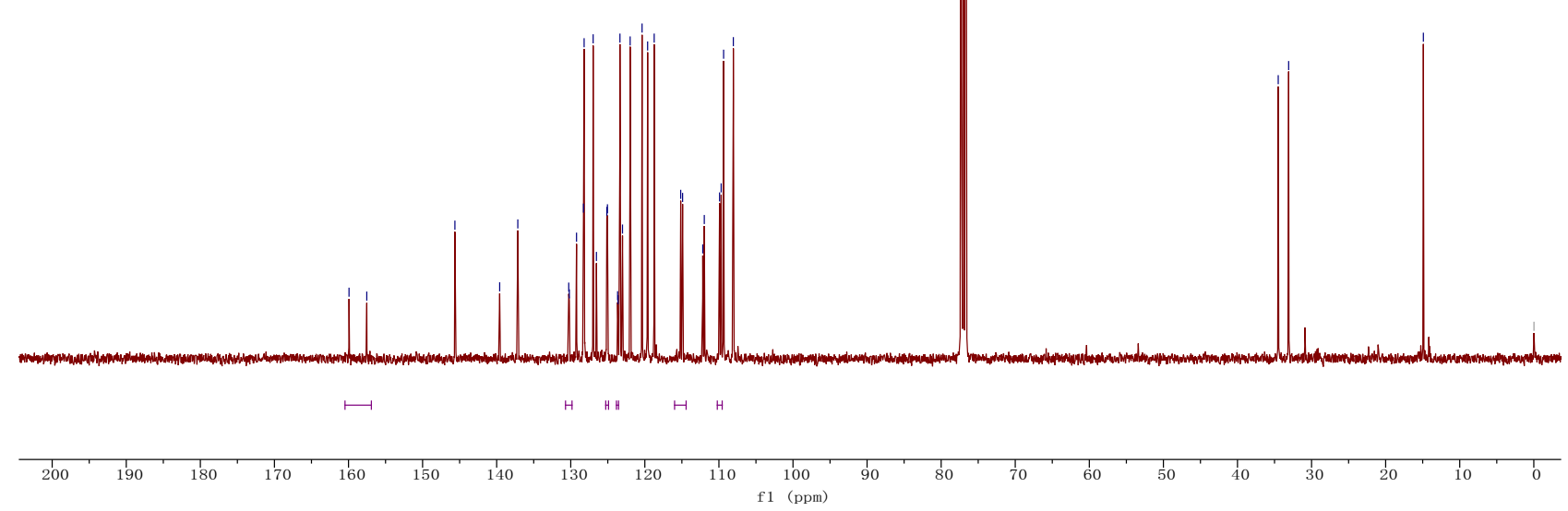




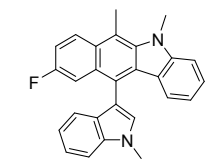

4e ( $\left.{ }^{19} \mathrm{~F} \mathrm{NMR}, 376 \mathrm{MHz}, \mathrm{CDCl}_{3}\right)$

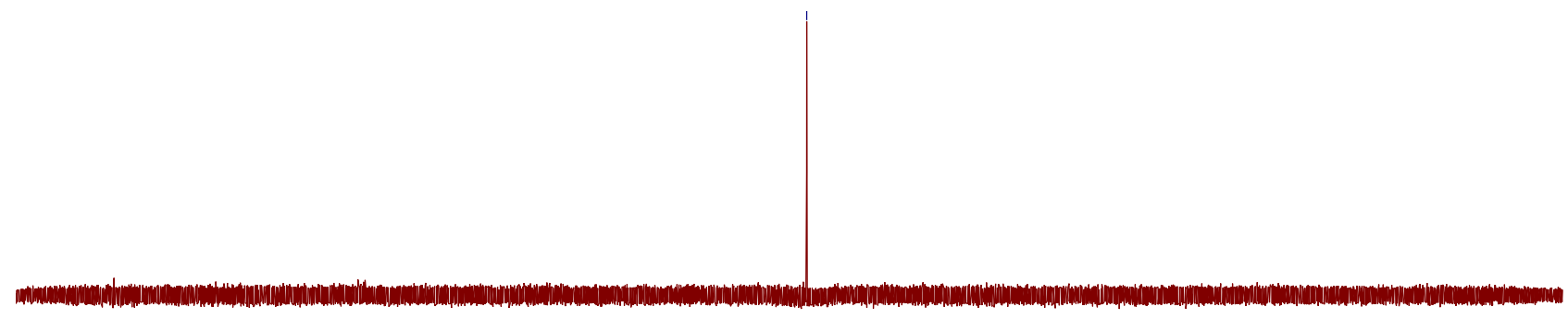

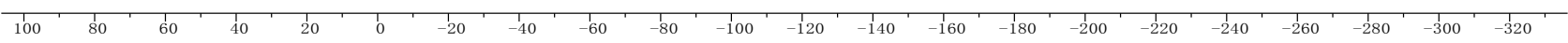




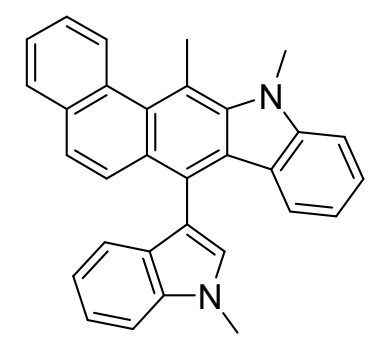

Compound 4f: Yield: $59 \mathrm{mg}, 70 \%$; a yellow solid; Mp: 138-141 ${ }^{\circ} \mathrm{C}$ (this isolated product containing a small of impurity due to its poor solubility); Eluent: $\mathrm{PE} / \mathrm{DCM}=3 / 1 .{ }^{1} \mathrm{H}$ NMR (400 $\left.\mathrm{MHz}, \mathrm{CDCl}_{3}, \mathrm{TMS}\right) \delta 8.72(\mathrm{~d}, J=7.8 \mathrm{~Hz}, 1 \mathrm{H}), 7.86(\mathrm{~d}, J=7.3 \mathrm{~Hz}, 1 \mathrm{H}), 7.79(\mathrm{~d}, J=9.0 \mathrm{~Hz}, 1 \mathrm{H})$, $7.63-7.52(\mathrm{~m}, 3 \mathrm{H}), 7.43(\mathrm{~s}, 1 \mathrm{H}), 7.41(\mathrm{~s}, 1 \mathrm{H}), 7.33$ (t, $J=7.9 \mathrm{~Hz}, 1 \mathrm{H}), 7.23(\mathrm{~s}, 1 \mathrm{H}), 7.16(\mathrm{~d}, J=$ $8.0 \mathrm{~Hz}, 1 \mathrm{H}), 7.05-6.93(\mathrm{~m}, 1 \mathrm{H}), 6.89-6.83(\mathrm{~m}, 1 \mathrm{H}), 4.24(\mathrm{~s}, 3 \mathrm{H}), 4.03(\mathrm{~s}, 3 \mathrm{H}), 3.47(\mathrm{~s}, 3 \mathrm{H})$; ${ }^{13} \mathrm{C}\left\{{ }^{1} \mathrm{H}\right\}$-NMR (100 MHz, $\mathrm{CDCl}_{3}$, TMS) $\delta_{\mathrm{C}} 145.2,143.5,137.0,133.4,131.2,130.6,129.2,128.6$, $128.4,128.1,127.8,126.2,126.0,125.7,124.5,124.2,124.0,123.7,123.5,122.9,121.9,120.6$, 119.6, 119.1, 115.3, 112.7, 109.3, 108.7, 34.8, 33.1, 23.1; IR (neat): v 2951, 2922, 2853, 1579, 1465, 1238, 820, $740 \mathrm{~cm}^{-1}$; HRMS (ESI-FTMS) m/z: $[\mathrm{M}+\mathrm{H}]^{+}$Calcd for $\mathrm{C}_{31} \mathrm{H}_{25} \mathrm{~N}_{2}$ 425.2012; found 425.2013. 


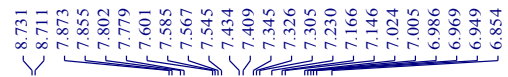

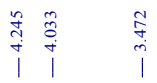
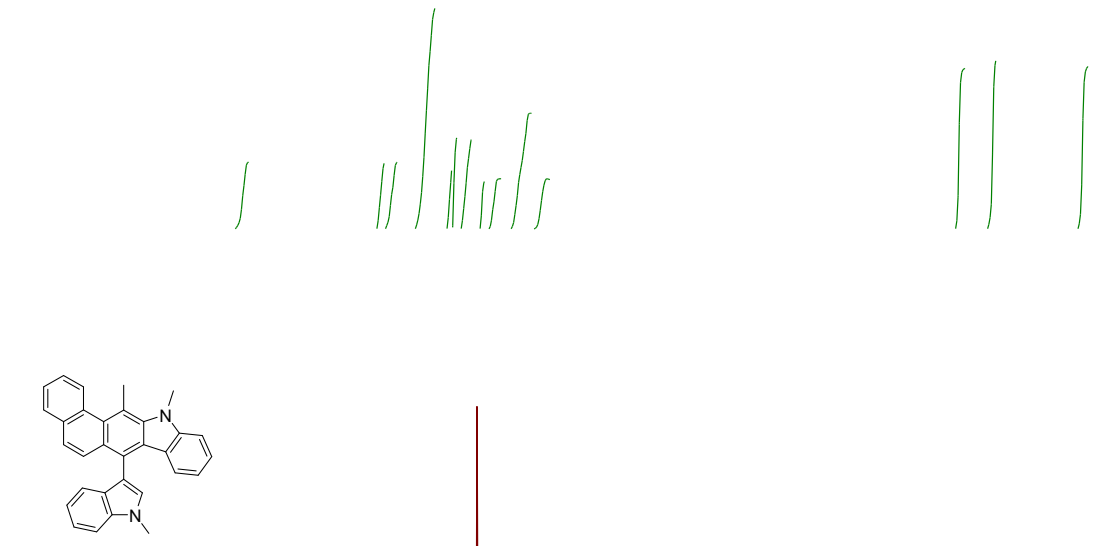

4f ( $\left.{ }^{1} \mathrm{H} \mathrm{NMR}, 400 \mathrm{MHz}, \mathrm{CDCl}_{3}\right)$
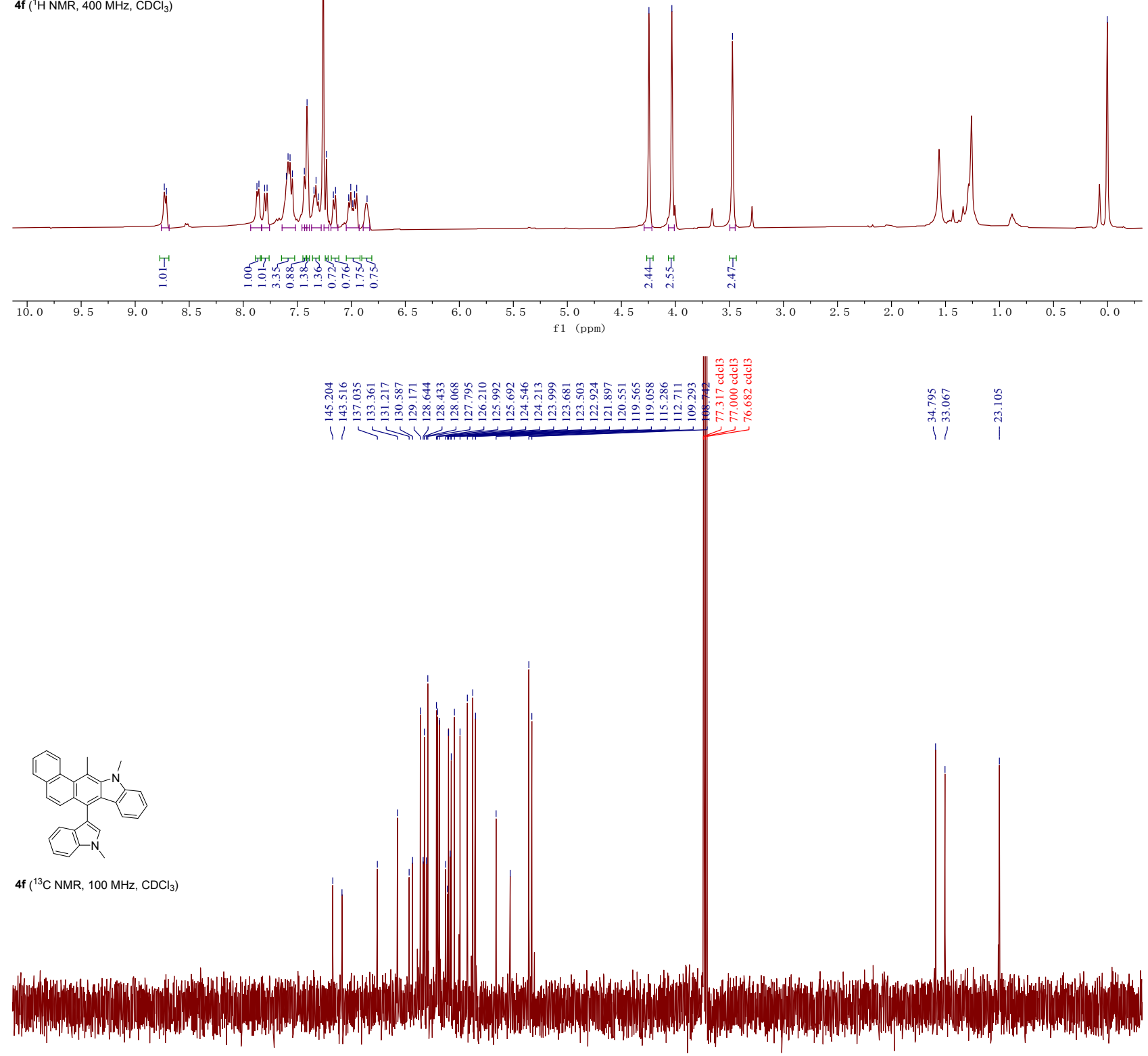

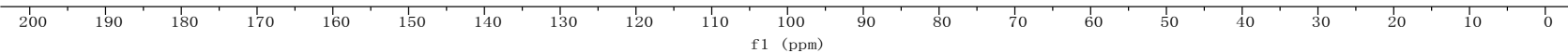




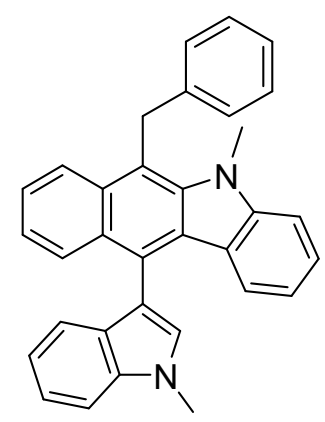

Compound 5a: Yield: 76 mg, 84\%; a yellow solid; Mp: $>200{ }^{\circ} \mathrm{C}$; Eluent: PE/DCM =3/1. ${ }^{1} \mathrm{H}$ NMR (400 MHz, $\left.\mathrm{CDCl}_{3}, \mathrm{TMS}\right) \delta 8.04(\mathrm{~d}, J=8.6 \mathrm{~Hz}, 1 \mathrm{H}), 7.94(\mathrm{dd}, J=8.6,1.2 \mathrm{~Hz}, 1 \mathrm{H}), 7.53(\mathrm{~d}, J=8.3$ $\mathrm{Hz}, 1 \mathrm{H}), 7.43-7.36(\mathrm{~m}, 1 \mathrm{H}), 7.36-7.30(\mathrm{~m}, 2 \mathrm{H}), 7.30-7.27$ (m, 4H), $7.23-7.16(\mathrm{~m}, 5 \mathrm{H}), 7.00$ (ddd, $J=8.0,7.0,1.0 \mathrm{~Hz}, 1 \mathrm{H}), 6.97-6.94(\mathrm{~m}, 1 \mathrm{H}), 6.79(\mathrm{ddd}, J=8.0,7.0,1.0 \mathrm{~Hz}, 1 \mathrm{H}), 5.06(\mathrm{~s}$, 2H), $4.00(\mathrm{~s}, 3 \mathrm{H}), 3.94(\mathrm{~s}, 3 \mathrm{H}) ;{ }^{13} \mathrm{C}\left\{{ }^{1} \mathrm{H}\right\}-\mathrm{NMR}\left(100 \mathrm{MHz}, \mathrm{CDCl}_{3}, \mathrm{TMS}\right) \delta_{\mathrm{C}} 145.1,141.5,140.3$, $137.1,132.5,129.6,128.7,128.5,128.30,128.29,127.3,126.7,126.1,125.8,125.6,125.2,123.24$, $123.18,123.1,122.2,121.9,120.6,119.6,118.7,112.6,112.4,109.3,107.9,33.7,33.4,33.1$; IR (neat): $v$ 3053, 1597, 1480, 1382, 1240, 982, 751, $736 \mathrm{~cm}^{-1}$; HRMS (ESI-FTMS) m/z: [M+H] ${ }^{+}$ Calcd for $\mathrm{C}_{33} \mathrm{H}_{27} \mathrm{~N}_{2} 451.2169$; found 451.2168. 

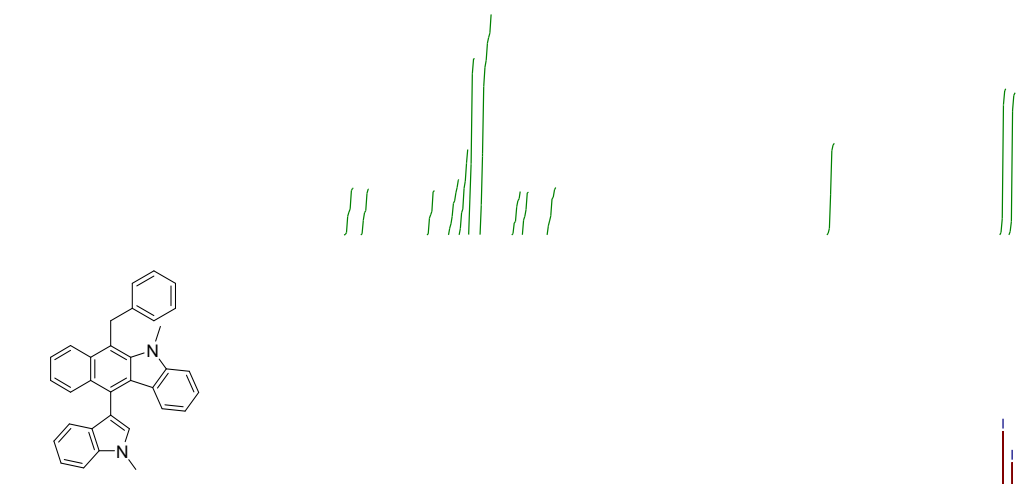

5a ( $\left.{ }^{1} \mathrm{H} \mathrm{NMR}, 400 \mathrm{MHz}, \mathrm{CDCl}\right)$
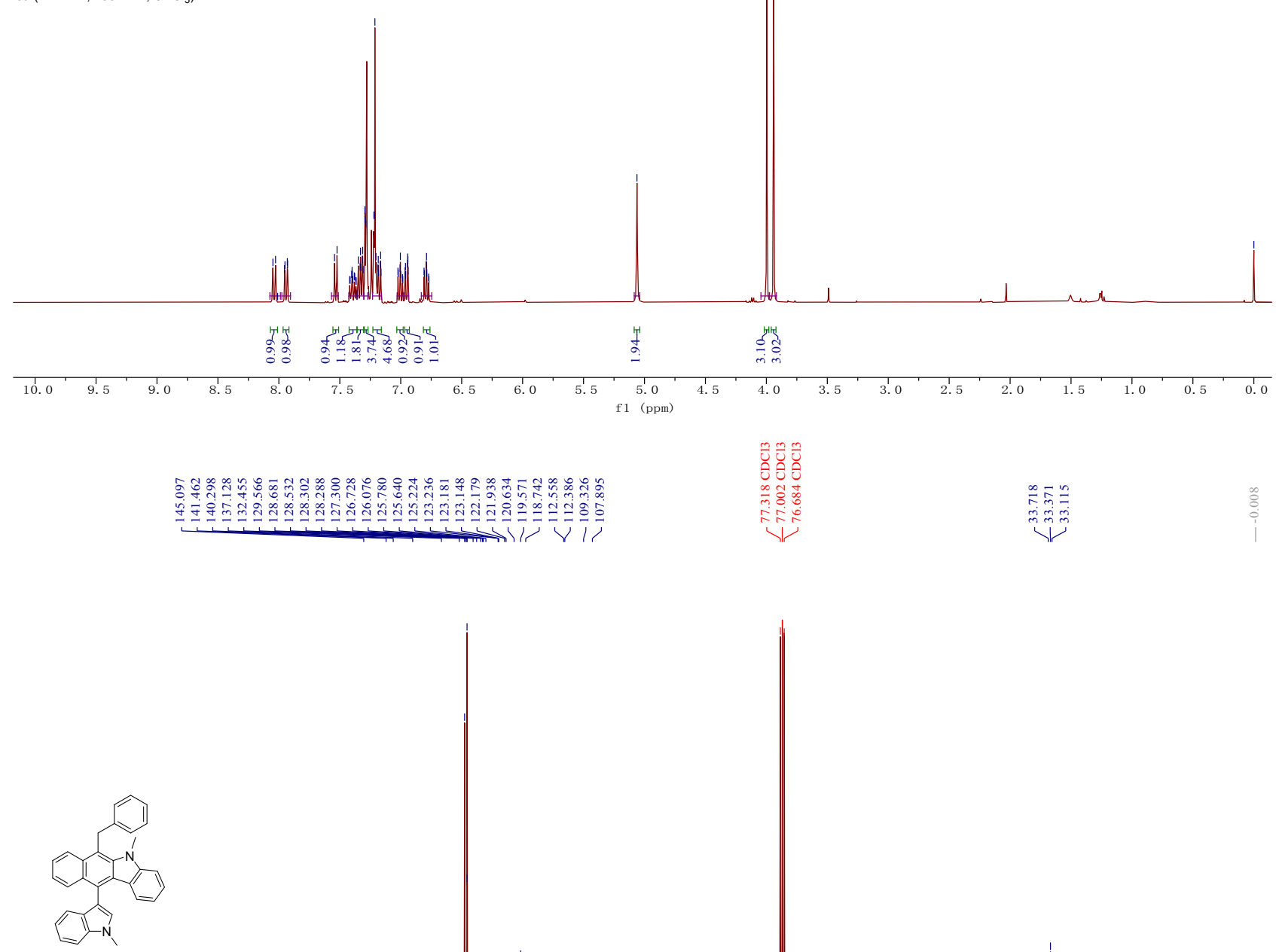

5a $\left({ }^{13} \mathrm{C} \mathrm{NMR}, 100 \mathrm{MHz}, \mathrm{CDCl}_{3}\right)$ 


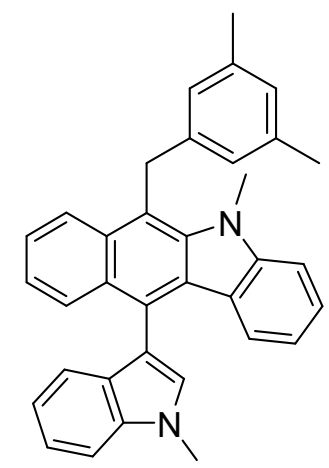

Compound 5b: Yield: $69 \mathrm{mg}$, 73\%; a yellow solid; Mp: 151-152 ${ }^{\circ} \mathrm{C}$; Eluent: PE/DCM = 3/1. ${ }^{1} \mathrm{H}$ NMR (400 MHz, $\left.\mathrm{CDCl}_{3}, \mathrm{TMS}\right) \delta 8.04(\mathrm{~d}, J=8.5 \mathrm{~Hz}, 1 \mathrm{H}), 7.94(\mathrm{~d}, J=8.5 \mathrm{~Hz}, 1 \mathrm{H}), 7.53(\mathrm{~d}, J=8.3$ Hz, 1H), $7.42-7.28$ (m, 3H), 7.23 (d, J=7.7 Hz, 2H), 7.19 (d, J=9.0 Hz, 2H), 7.03 - 6.94 (m, 2H), $6.89(\mathrm{~s}, 2 \mathrm{H}), 6.85(\mathrm{~s}, 1 \mathrm{H}), 6.79(\mathrm{t}, J=7.5 \mathrm{~Hz}, 1 \mathrm{H}), 4.96(\mathrm{~s}, 2 \mathrm{H}), 3.98(\mathrm{~s}, 3 \mathrm{H}), 3.94(\mathrm{~s}, 3 \mathrm{H}), 2.23$ $(\mathrm{s}, 6 \mathrm{H}) ;{ }^{13} \mathrm{C}\left\{{ }^{1} \mathrm{H}\right\}-\mathrm{NMR}\left(100 \mathrm{MHz}, \mathrm{CDCl}_{3}\right.$, TMS) $\delta_{\mathrm{C}} 145.0,141.5,140.3,138.1,137.1,132.6,129.5$, $128.5,128.3,127.8,127.2,126.7,126.0,125.6,125.5,125.1,123.3,123.2,123.1,122.1,121.9$, 120.6, 119.5, 118.7, 112.7, 112.4, 109.3, 107.9, 33.6, 33.3, 33.0, 21.3; IR (neat): v 3066, 2911, 1597, 1471, 1321, 1142, 906, $738 \mathrm{~cm}^{-1}$; HRMS (ESI-TOF) m/z: $[\mathrm{M}+\mathrm{H}]^{+}$Calcd for $\mathrm{C}_{35} \mathrm{H}_{31} \mathrm{~N}_{2} 479.2482$; found 479.2483. 


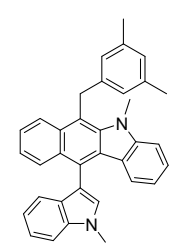

II I $/\|/\|$

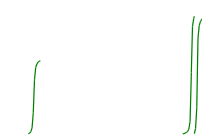

5b ( ${ }^{1} \mathrm{H} \mathrm{NMR}, 400 \mathrm{MHz}, \mathrm{CDCl}_{3}$ )
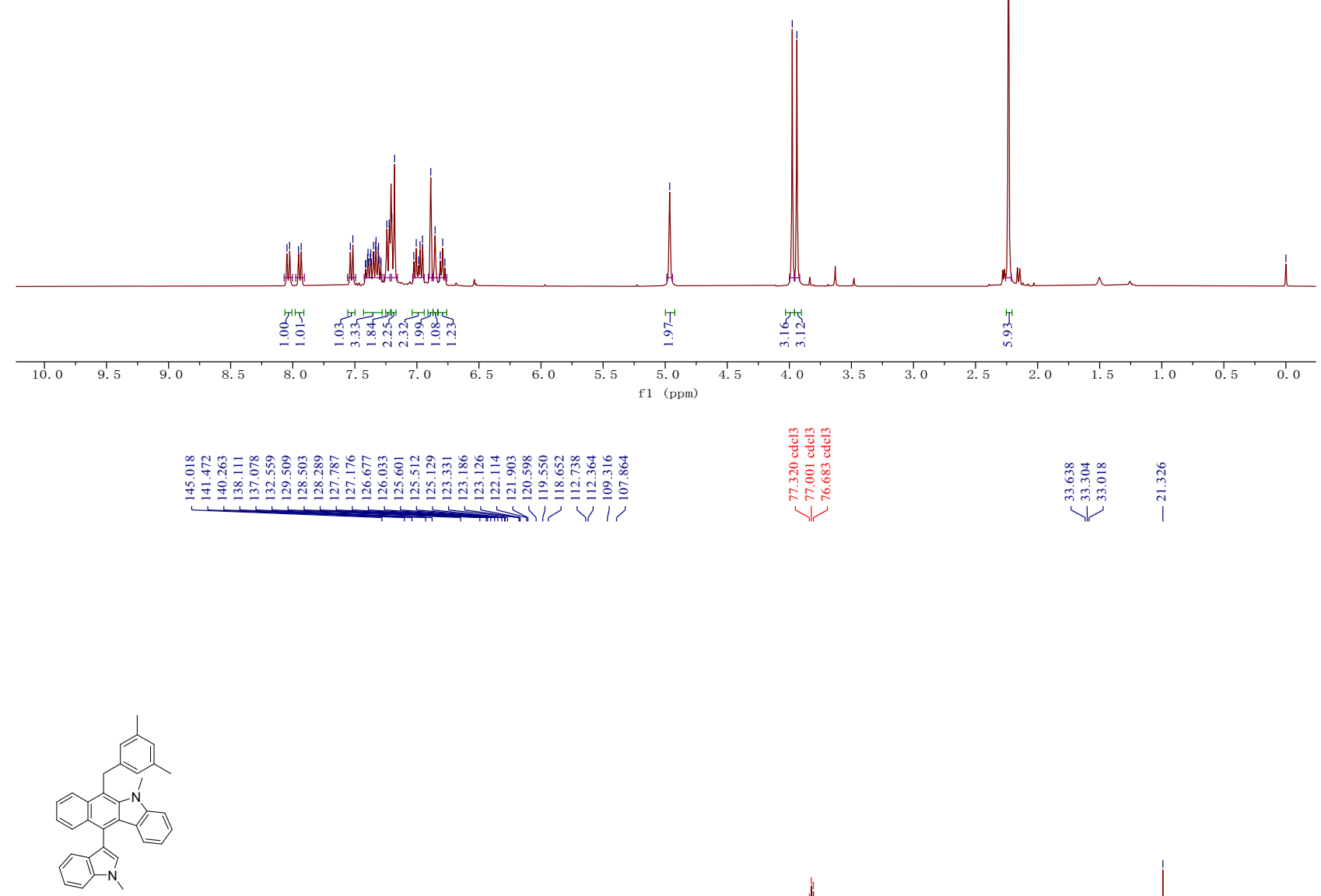

5b $\left({ }^{13} \mathrm{C} \mathrm{NMR}, 100 \mathrm{MHz}, \mathrm{CDCl}_{3}\right)$
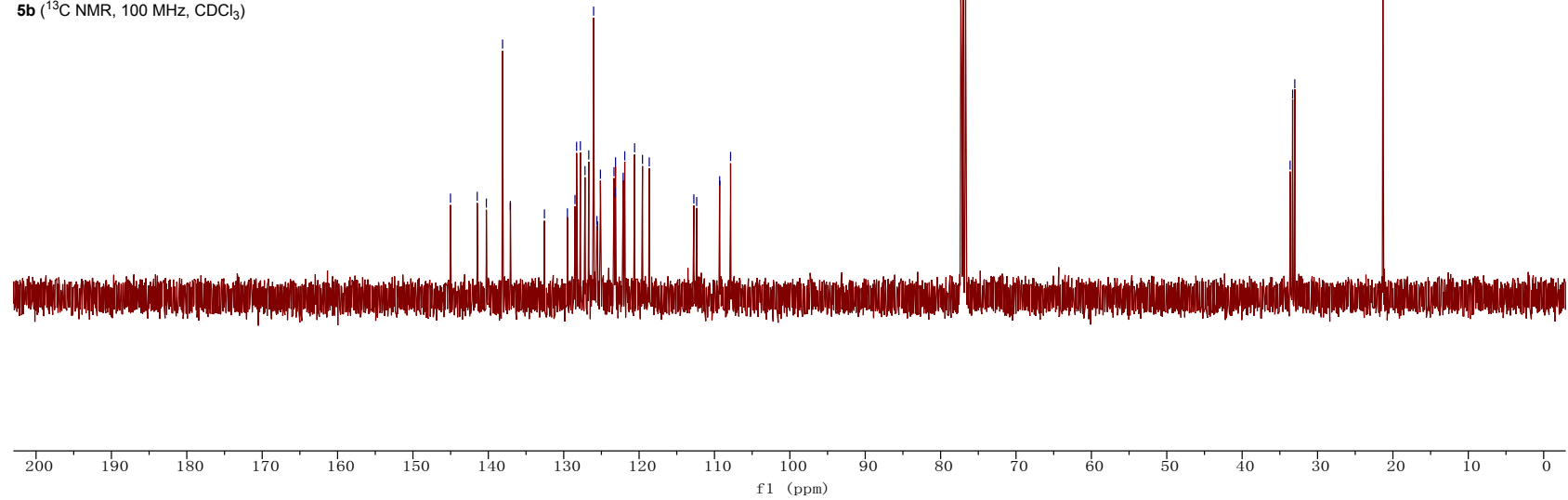


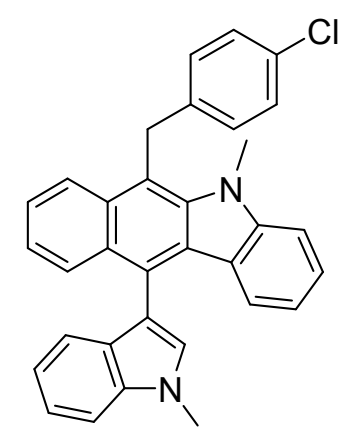

Compound 5c: Yield: 74 mg, 76\%; a yellow solid; Mp: $>200{ }^{\circ} \mathrm{C}$; Eluent: PE/DCM =1/1. ${ }^{1} \mathrm{H}$ NMR (400 MHz, $\mathrm{CDCl}_{3}$, TMS) $\delta 8.00-7.91(\mathrm{~m}, 2 \mathrm{H}), 7.54(\mathrm{~d}, J=8.3 \mathrm{~Hz}, 1 \mathrm{H}), 7.43-7.29(\mathrm{~m}, 3 \mathrm{H}), 7.27$ $-7.15(\mathrm{~m}, 8 \mathrm{H}), 7.01$ (t, $J=7.4 \mathrm{~Hz}, 1 \mathrm{H}), 6.95(\mathrm{~d}, J=6.9 \mathrm{~Hz}, 1 \mathrm{H}), 6.85-6.76(\mathrm{~m}, 1 \mathrm{H}), 5.00(\mathrm{~s}, 2 \mathrm{H})$, $4.00(\mathrm{~s}, 3 \mathrm{H}), 3.91(\mathrm{~s}, 3 \mathrm{H}) ;{ }^{13} \mathrm{C}\left\{{ }^{1} \mathrm{H}\right\}-\mathrm{NMR}\left(100 \mathrm{MHz}, \mathrm{CDCl}_{3}, \mathrm{TMS}\right) \delta_{\mathrm{C}} 145.0,140.3,139.9,137.1$, $132.2,131.9,129.61,129.56,128.8,128.5,128.3,127.4,126.8,126.1,125.7,125.4,123.2,122.8$, 122.3, 122.0, 120.6, 119.6, 118.9, 112.2, 111.9, 109.4, 107.9, 33.4, 33.13, 33.08; IR (neat): v 3055, 3029, 2917, 1596, 1881, 1241, 760, $741 \mathrm{~cm}^{-1}$; HRMS (ESI-FTMS) m/z: $[\mathrm{M}+\mathrm{H}]^{+}$Calcd for $\mathrm{C}_{33} \mathrm{H}_{26} \mathrm{~N}_{2} \mathrm{Cl} 485.1779$; found 485.1780 . 

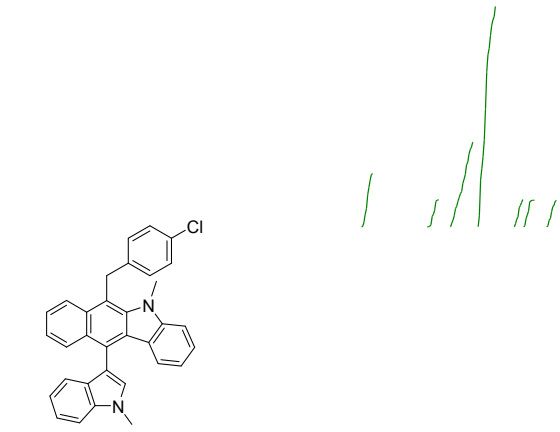

5c ( $\left.{ }^{1} \mathrm{H} \mathrm{NMR}, 400 \mathrm{MHz}, \mathrm{CDCl}_{3}\right)$

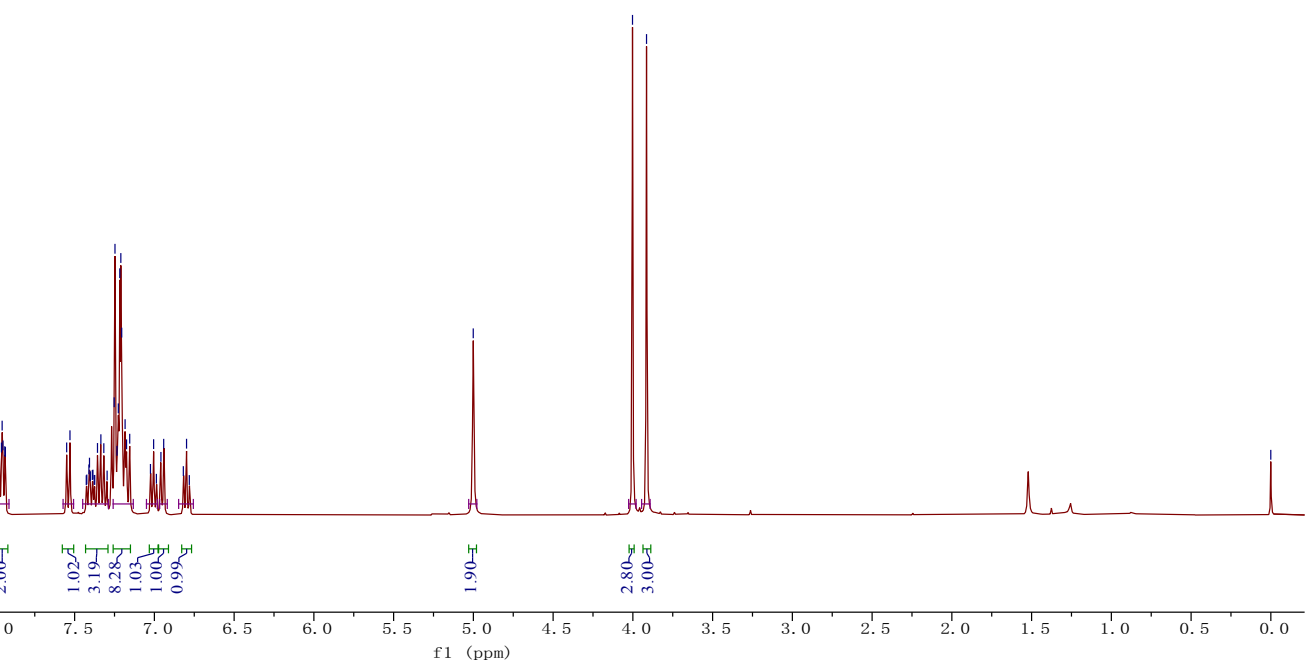

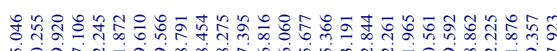

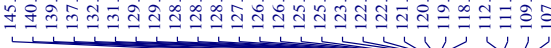

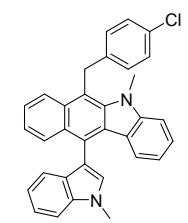

5c $\left({ }^{13} \mathrm{C} \mathrm{NMR}, 100 \mathrm{MHz}, \mathrm{CDCl}_{3}\right)$

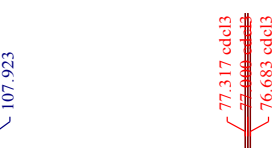

每

लें
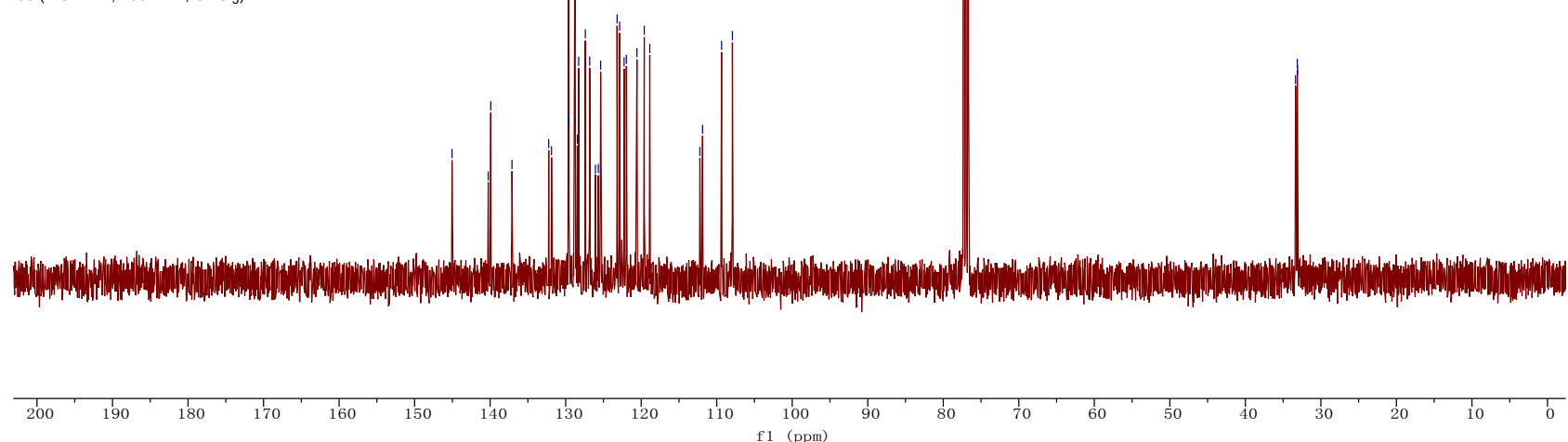


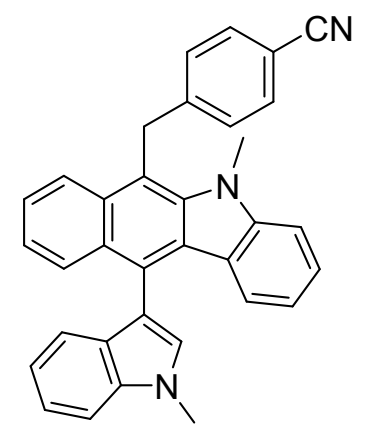

Compound 5d: Yield: $45 \mathrm{mg}$, 47\%; a yellow solid; Mp: 101-103 ${ }^{\circ} \mathrm{C}$; Eluent: PE/EA = 1/1. ${ }^{1} \mathrm{H}$ NMR (400 MHz, $\left.\mathrm{CDCl}_{3}, \mathrm{TMS}\right) \delta 7.96(\mathrm{~d}, J=8.5 \mathrm{~Hz}, 1 \mathrm{H}), 7.86(\mathrm{~d}, J=8.5 \mathrm{~Hz}, 1 \mathrm{H}), 7.54$ (t, $J=8.0$ $\mathrm{Hz}, 3 \mathrm{H}), 7.44-7.28(\mathrm{~m}, 5 \mathrm{H}), 7.24(\mathrm{~d}, J=8.5 \mathrm{~Hz}, 1 \mathrm{H}), 7.20-7.18(\mathrm{~m}, 2 \mathrm{H}), 7.15$ (d, $J=7.9 \mathrm{~Hz}$, 1H), $7.04-6.97(\mathrm{~m}, 1 \mathrm{H}), 6.95(\mathrm{~d}, J=7.8 \mathrm{~Hz}, 1 \mathrm{H}), 6.80(\mathrm{t}, J=7.5 \mathrm{~Hz}, 1 \mathrm{H}), 5.03(\mathrm{~s}, 2 \mathrm{H}), 3.98$ (s, 3H), $3.84(\mathrm{~s}, 3 \mathrm{H}) ;{ }^{13} \mathrm{C}\left\{{ }^{1} \mathrm{H}\right\}-\mathrm{NMR}\left(100 \mathrm{MHz}, \mathrm{CDCl}_{3}\right.$, TMS) $\delta_{\mathrm{C}} 147.1,144.9,140.2,137.0,132.5$, $132.0,129.5,129.0,128.3,128.2,127.5,126.9,126.4,125.7,125.5,123.2,123.0,122.4,122.3$, 122.0, 120.4, 119.6, 119.0, 118.9, 112.0, 110.6, 110.0, 109.4, 107.9, 33.7, 33.3, 33.1; IR (neat): $v$ 3050, 2927, 2228, 1595, 1479, 1241, 1117, $736 \mathrm{~cm}^{-1}$; HRMS (EI-TOF) m/z: [M] Calcd for $\mathrm{C}_{34} \mathrm{H}_{25} \mathrm{~N}_{3}$ 475.2043; found 475.2046. 

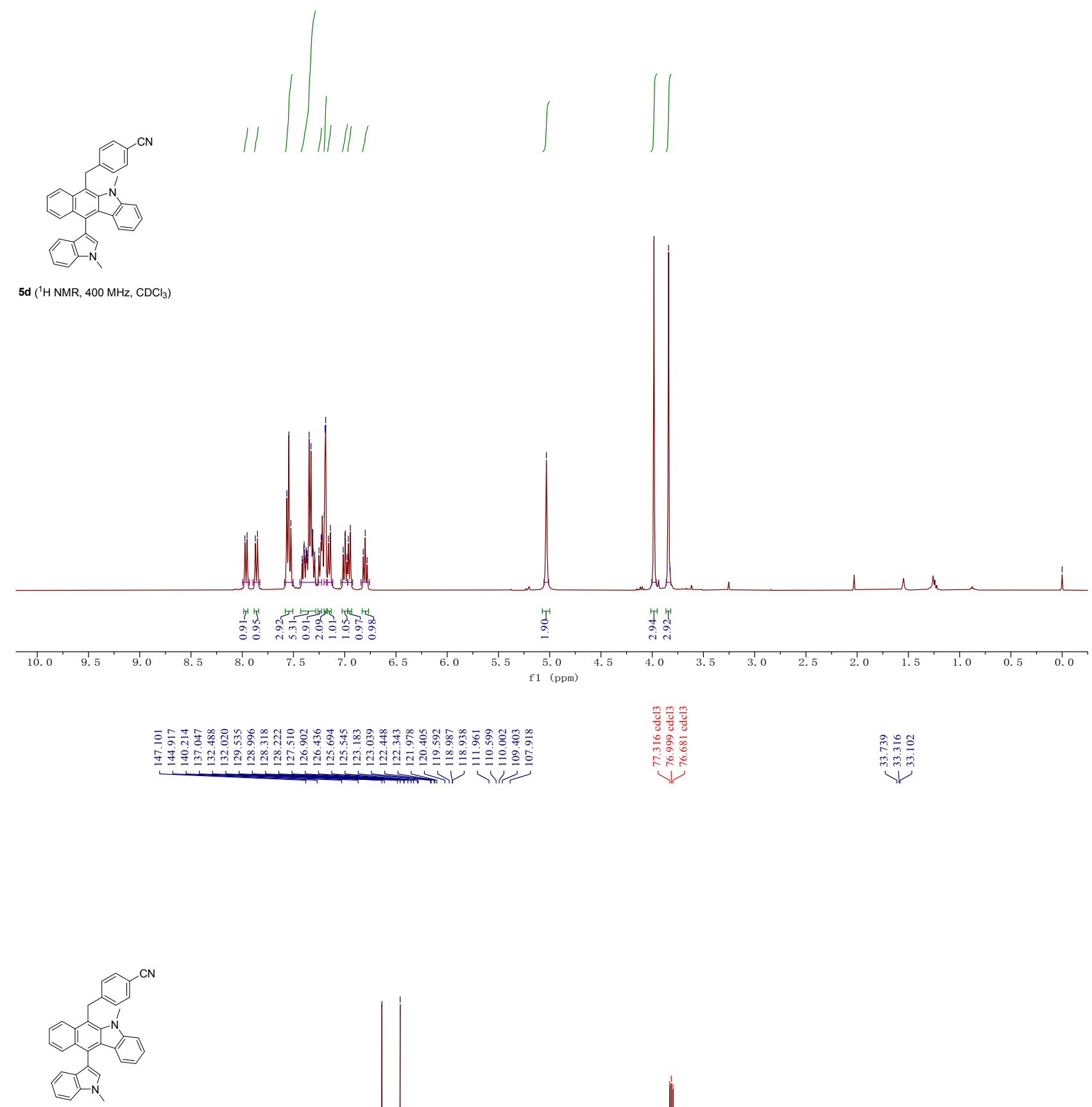

5d $\left({ }^{13} \mathrm{C} \mathrm{NMR}, 100 \mathrm{MHz}, \mathrm{CDCl}_{3}\right)$
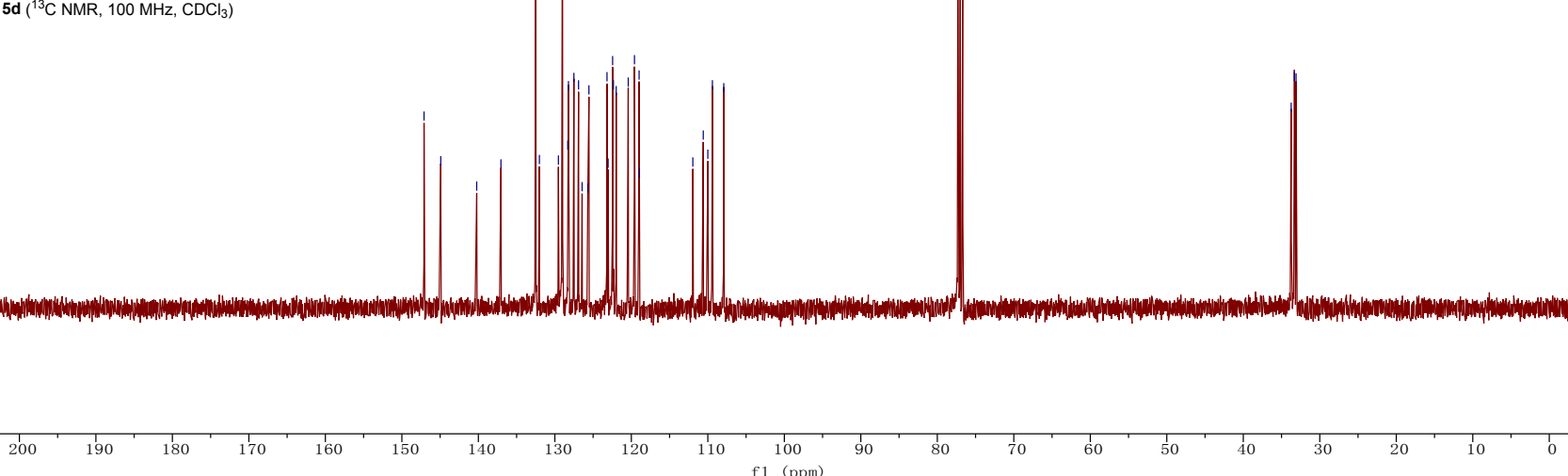


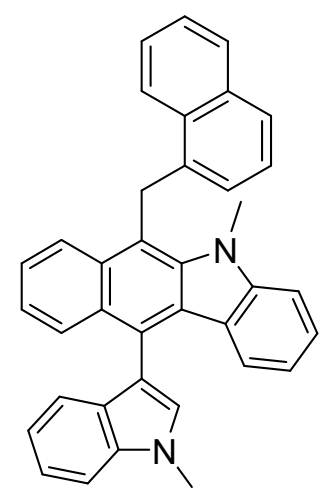

Compound 5e: Yield: $68 \mathrm{mg}, 68 \%$; a yellow solid; Mp: $>200{ }^{\circ} \mathrm{C}$; Eluent: PE/DCM =2/1. ${ }^{1} \mathrm{H}$ NMR $\left(400 \mathrm{MHz}, \mathrm{CD}_{2} \mathrm{Cl}_{2}, \mathrm{TMS}\right) \delta 8.42(\mathrm{~d}, J=8.4 \mathrm{~Hz}, 1 \mathrm{H}), 7.91(\mathrm{~d}, J=8.0 \mathrm{~Hz}, 1 \mathrm{H}), 7.86(\mathrm{~d}, J=8.9 \mathrm{~Hz}$, 2H), $7.74-7.62(\mathrm{~m}, 2 \mathrm{H}), 7.59-7.48(\mathrm{~m}, 2 \mathrm{H}), 7.29-7.22(\mathrm{~m}, 4 \mathrm{H}), 7.19-7.11(\mathrm{~m}, 3 \mathrm{H}), 7.02(\mathrm{~d}, J$ $=8.1 \mathrm{~Hz}, 1 \mathrm{H}), 6.93(\mathrm{~d}, J=7.4 \mathrm{~Hz}, 2 \mathrm{H}), 6.86(\mathrm{~d}, J=7.9 \mathrm{~Hz}, 1 \mathrm{H}), 6.70(\mathrm{t}, J=7.5 \mathrm{~Hz}, 1 \mathrm{H}), 5.36(\mathrm{~s}$, 2H), $3.96(\mathrm{~s}, 3 \mathrm{H}), 3.75(\mathrm{~s}, 3 \mathrm{H}) ;{ }^{13} \mathrm{C}\left\{{ }^{1} \mathrm{H}\right\}-\mathrm{NMR}\left(100 \mathrm{MHz}, \mathrm{CD}_{2} \mathrm{Cl}_{2} \mathrm{TMS}\right) \delta_{\mathrm{C}}$ 145.2, 140.5, 137.3, $137.1,133.8,132.6,131.7,129.6,128.8,128.5,127.1,126.84,126.79,126.6,126.4,125.9,125.8$, 125.6, 125.2, 123.14, 123.06, 122.9, 122.1, 121.9, 120.0, 119.4, 118.6, 112.3, 112.0, 109.6, 108.0, 33.0, 32.8, 31.0; IR (neat): v 3066, 2964, 2919, 1597, 1482, 1158, 796, $732 \mathrm{~cm}^{-1}$; HRMS (ESI-TOF) $\mathrm{m} / \mathrm{z}:[\mathrm{M}+\mathrm{H}]^{+}$Calcd for $\mathrm{C}_{37} \mathrm{H}_{29} \mathrm{~N}_{2}$ 501.2325; found 501.2327. 

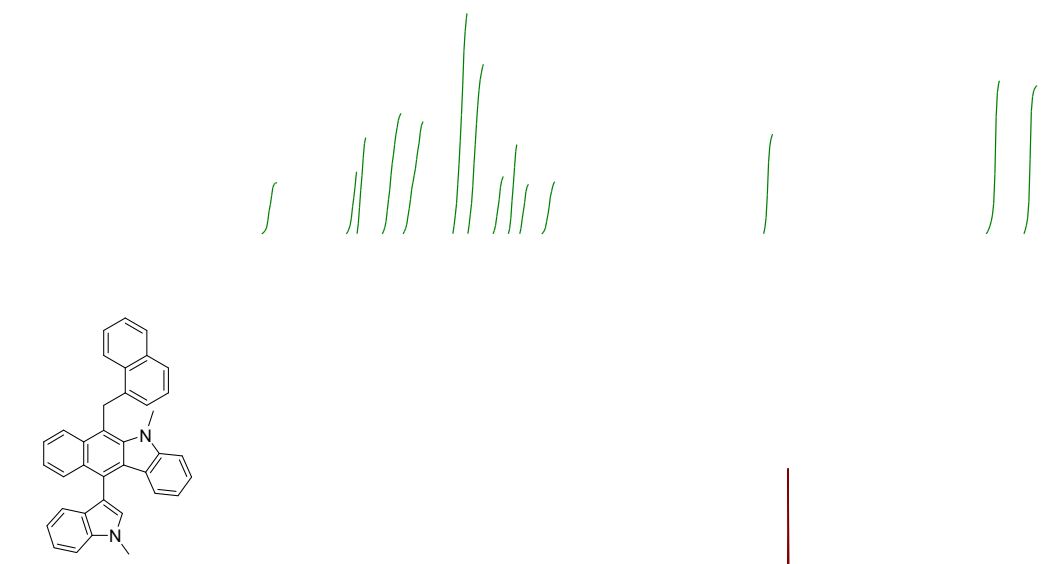

5e ( ${ }^{1} \mathrm{H} \mathrm{NMR}, 400 \mathrm{MHz}, \mathrm{CD}_{2} \mathrm{Cl}_{2}$ )

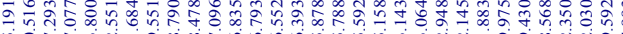

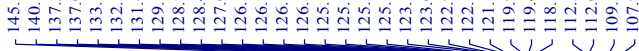
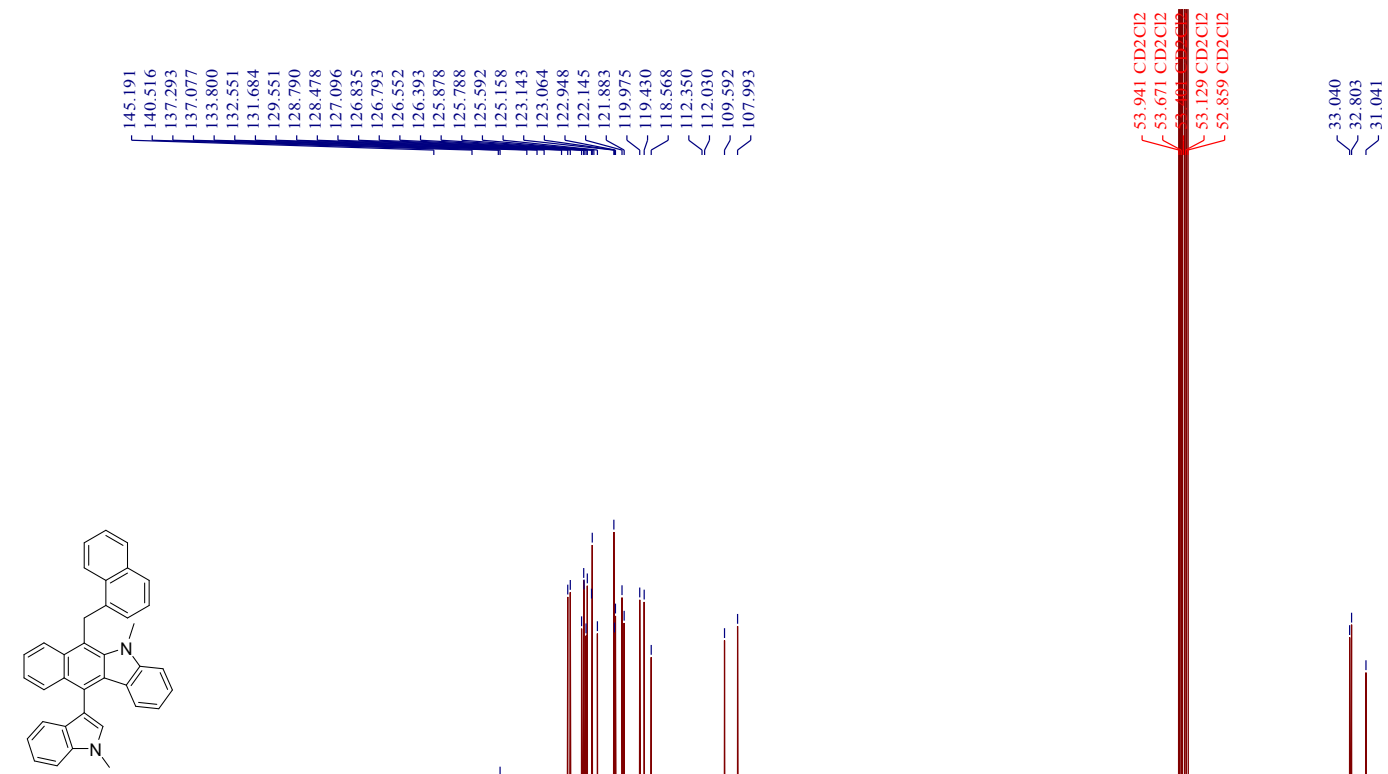

5e $\left({ }^{13} \mathrm{C} \mathrm{NMR}, 100 \mathrm{MHz}, \mathrm{CD}_{2} \mathrm{Cl}_{2}\right)$

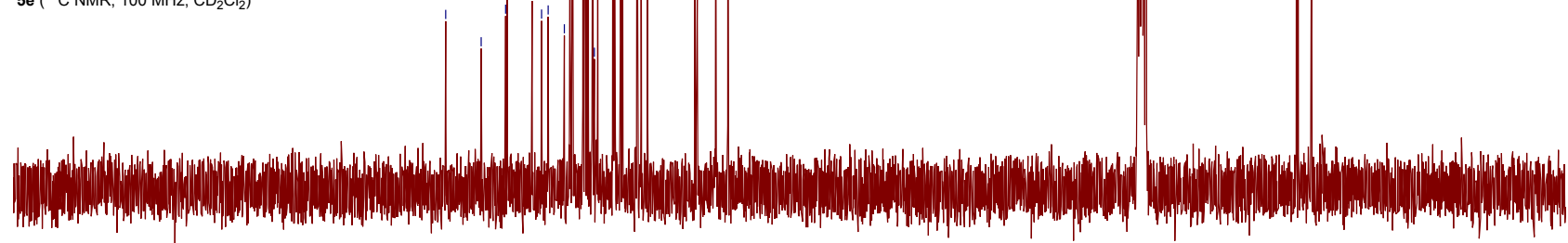

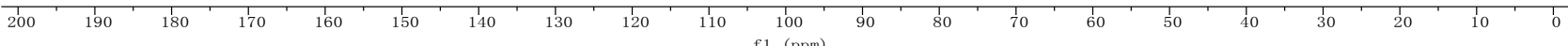




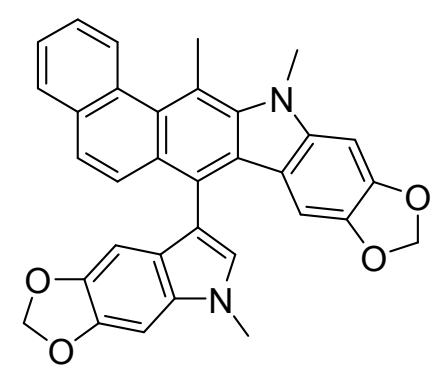

Compound 6: Yield: $26 \mathrm{mg}, 26 \%$; a yellow solid; Mp: $>200{ }^{\circ} \mathrm{C}$; Eluent: PE/DCM =2/1. ${ }^{1} \mathrm{H}$ NMR $\left(400 \mathrm{MHz}, \mathrm{CD}_{2} \mathrm{Cl}_{2}, \mathrm{TMS}\right) \delta 8.67(\mathrm{~d}, J=9.1 \mathrm{~Hz}, 1 \mathrm{H}), 7.84(\mathrm{dd}, J=6.9,2.3 \mathrm{~Hz}, 1 \mathrm{H}), 7.75(\mathrm{~d}, J=9.1$ $\mathrm{Hz}, 1 \mathrm{H}), 7.56$ (ddd, $J=7.0,5.0,1.8 \mathrm{~Hz}, 2 \mathrm{H}), 7.41$ (d, $J=9.0 \mathrm{~Hz}, 1 \mathrm{H}), 7.03(\mathrm{~s}, 1 \mathrm{H}), 6.95$ (s, 1H), $6.89(\mathrm{~s}, 1 \mathrm{H}), 6.45(\mathrm{~s}, 1 \mathrm{H}), 6.40(\mathrm{~s}, 1 \mathrm{H}), 5.95-5.85(\mathrm{~m}, 4 \mathrm{H}), 4.14(\mathrm{~s}, 3 \mathrm{H}), 3.91(\mathrm{~s}, 3 \mathrm{H}), 3.41(\mathrm{~s}, 3 \mathrm{H})$; ${ }^{13} \mathrm{C}\left\{{ }^{1} \mathrm{H}\right\}-\mathrm{NMR}\left(100 \mathrm{MHz}, \mathrm{CD}_{2} \mathrm{Cl}_{2} \mathrm{TMS}\right) \delta_{\mathrm{C}} 147.5,145.3,143.6,143.1,141.7,141.4,133.3,132.3$, $130.7,130.0,129.0,128.0,127.8,126.7,125.8,125.5,124.4,124.0,123.5,123.2,122.7,116.6$, 115.5, 112.8, 102.4, 100.8, 100.6, 98.8, 90.7, 90.4, 35.1, 33.4, 23.0; IR (neat): v 2872, 1469, 1447, 1333, 1224, 1099, 1036, $817 \mathrm{~cm}^{-1}$; HRMS (ESI-TOF) m/z: $[\mathrm{M}+\mathrm{H}]^{+}$Calcd for $\mathrm{C}_{33} \mathrm{H}_{25} \mathrm{~N}_{2} \mathrm{O}_{4} 513.1809$; found 513.1810 . 

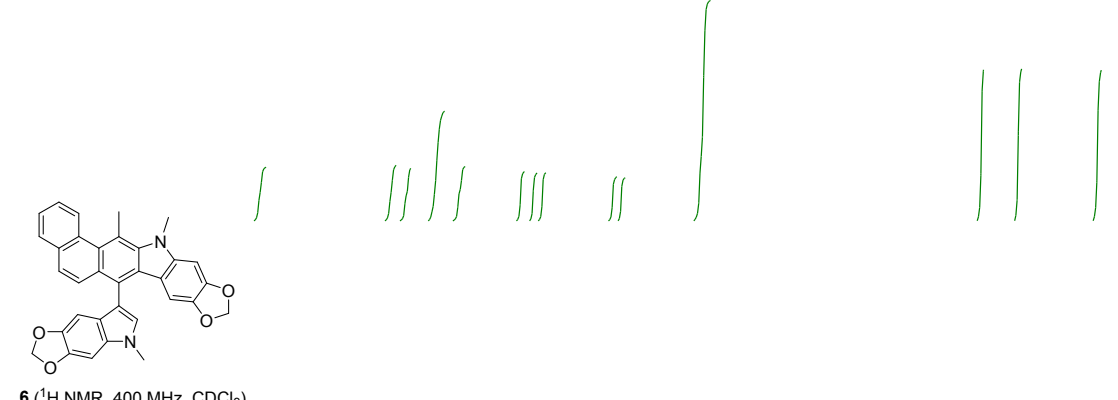

$6\left({ }^{1} \mathrm{H} \mathrm{NMR}, 400 \mathrm{MHz}, \mathrm{CDCl}_{3}\right)$
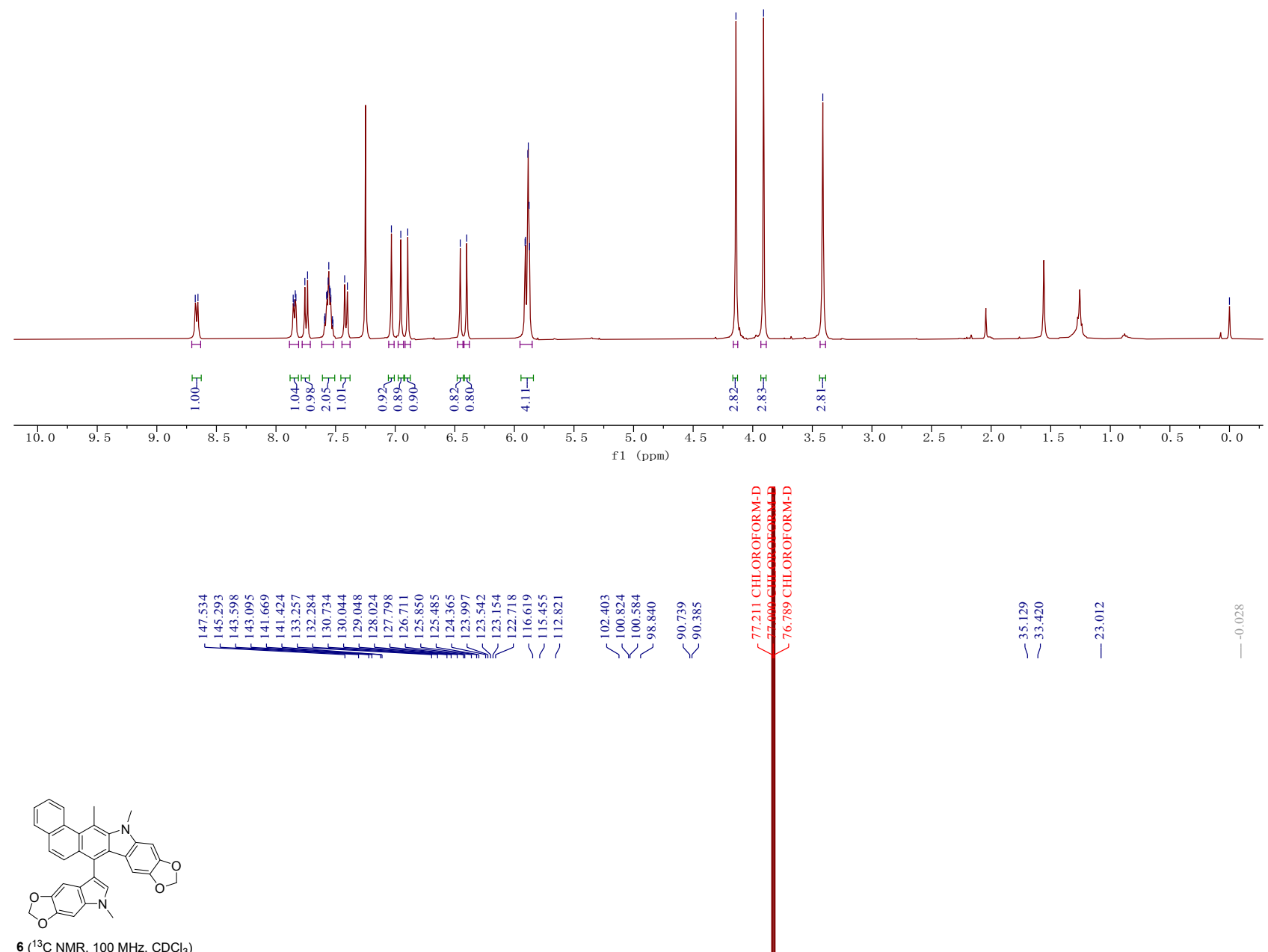

$6\left({ }^{13} \mathrm{C} \mathrm{NMR}, 100 \mathrm{MHz}, \mathrm{CDCl}_{3}\right)$ 


\section{Characterization Data of Substrates.}<smiles>C=C1OC(OC)c2ccccc21</smiles>

Compound 1a: Yield: $291 \mathrm{mg}, 90 \%$; this is a known compound and its spectroscopic data are consistent with those reported ones. ${ }^{1}{ }^{1} \mathrm{H}$ NMR (400 MHz, $\left.\mathrm{CDCl}_{3}, \mathrm{TMS}\right) \delta 7.55-7.48(\mathrm{~m}, 1 \mathrm{H}), 7.44$ $-7.37(\mathrm{~m}, 3 \mathrm{H}), 6.36(\mathrm{~s}, 1 \mathrm{H}), 4.63(\mathrm{~d}, J=2.4 \mathrm{~Hz}, 1 \mathrm{H}), 4.61(\mathrm{~d}, J=2.3 \mathrm{~Hz}, 1 \mathrm{H}), 3.45(\mathrm{~s}, 3 \mathrm{H})$; ${ }^{13} \mathrm{C}\left\{{ }^{1} \mathrm{H}\right\}$-NMR (100 MHz, $\mathrm{CDCl}_{3}$, TMS) $\delta_{\mathrm{C}} 159.0,137.9,134.2,129.8,129.3,123.0,120.4,106.3$, $80.6,54.4$. 

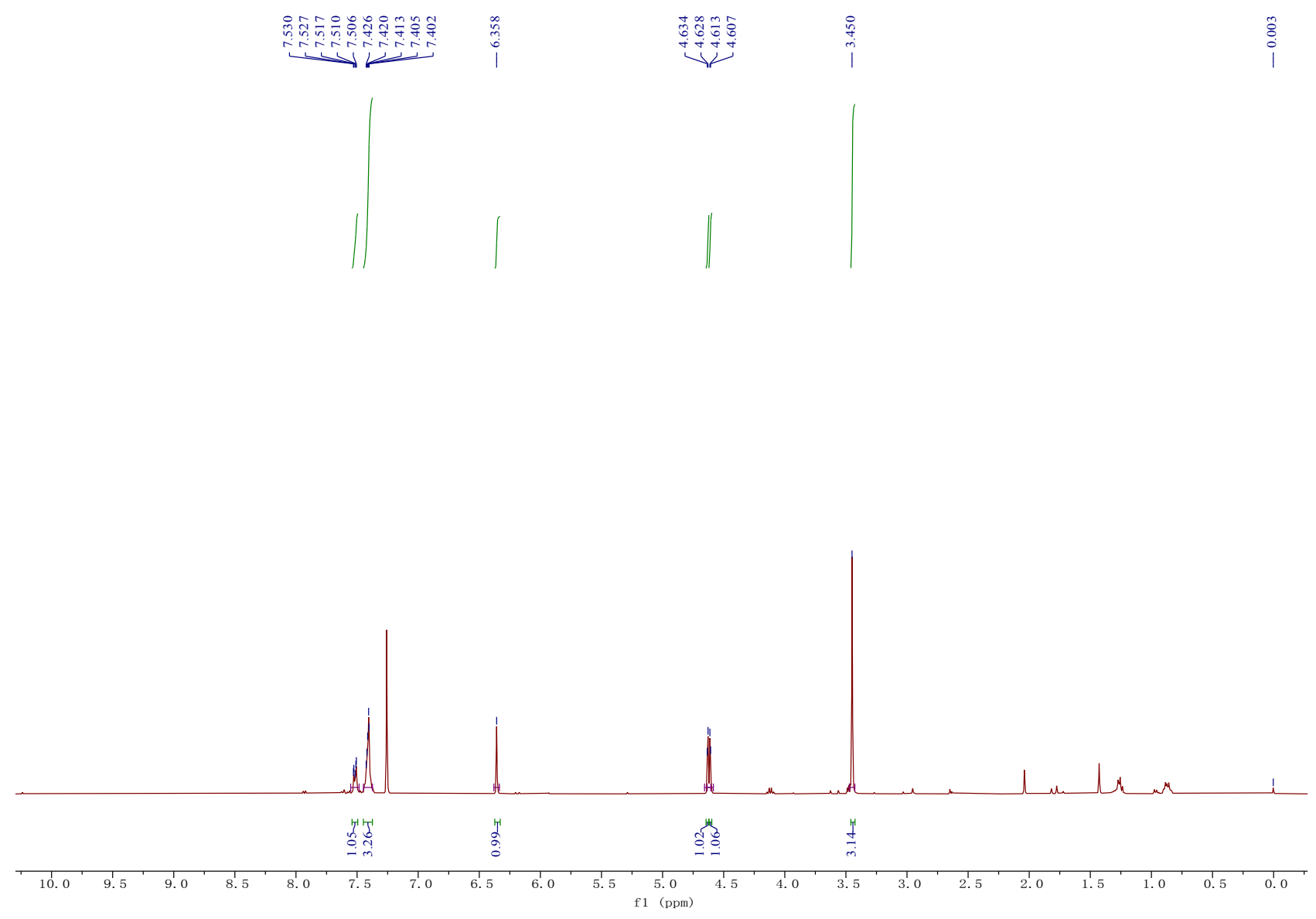

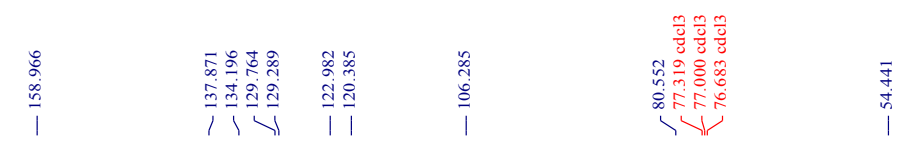

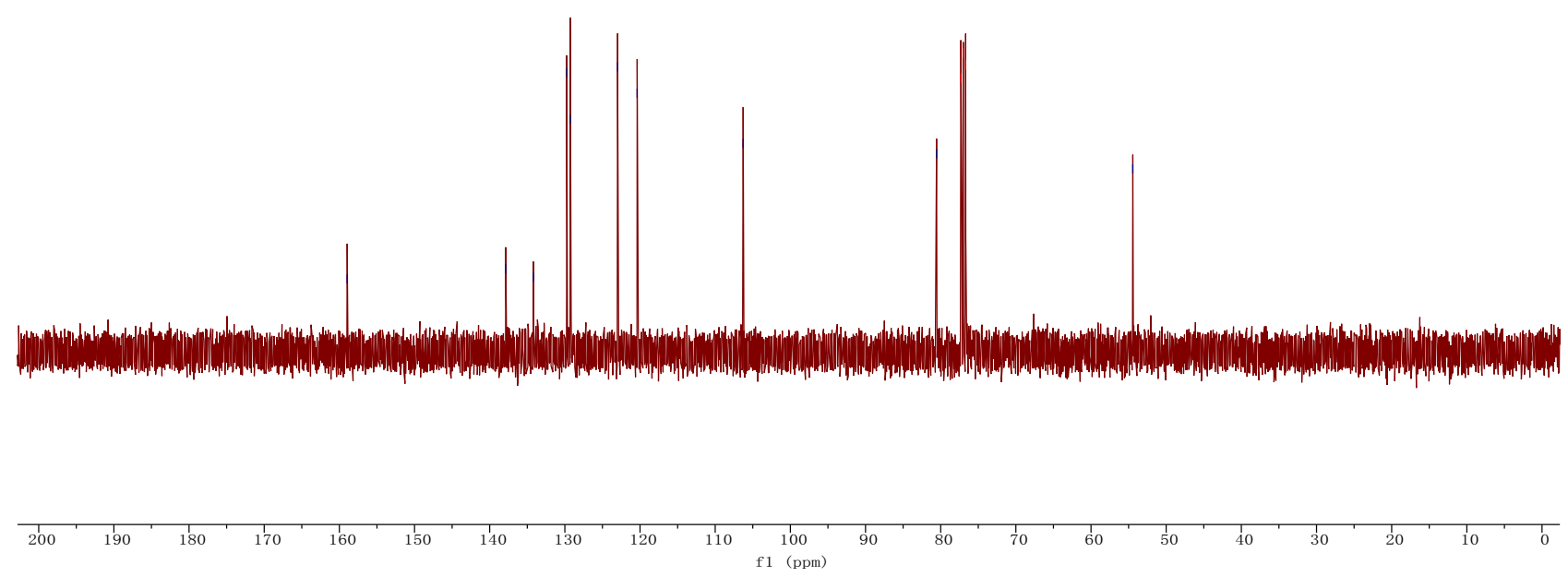




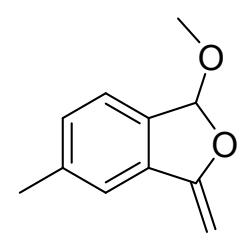

Compound 1b: Yield: $299 \mathrm{mg}, 85 \%$; ${ }^{1} \mathrm{H}$ NMR (400 MHz, $\mathrm{CDCl}_{3}$, TMS) $\delta 7.31$ (s, 1H), 7.29 (d, $J=$ $7.8 \mathrm{~Hz}, 1 \mathrm{H}), 7.20(\mathrm{~d}, J=7.6 \mathrm{~Hz}, 1 \mathrm{H}), 6.32(\mathrm{~s}, 1 \mathrm{H}), 4.59$ (d, $J=2.3 \mathrm{~Hz}, 1 \mathrm{H}), 4.57(\mathrm{~d}, J=2.3 \mathrm{~Hz}$, 1H), $3.42(\mathrm{~s}, 3 \mathrm{H}), 2.40(\mathrm{~s}, 3 \mathrm{H}) ;{ }^{13} \mathrm{C}\left\{{ }^{1} \mathrm{H}\right\}-\mathrm{NMR}\left(100 \mathrm{MHz}, \mathrm{CDCl}_{3}, \mathrm{TMS}\right) \delta_{\mathrm{C}} 159.0,139.8,135.3$, $134.4,130.4,122.7,120.6,106.2,80.1,54.2,21.4$. 

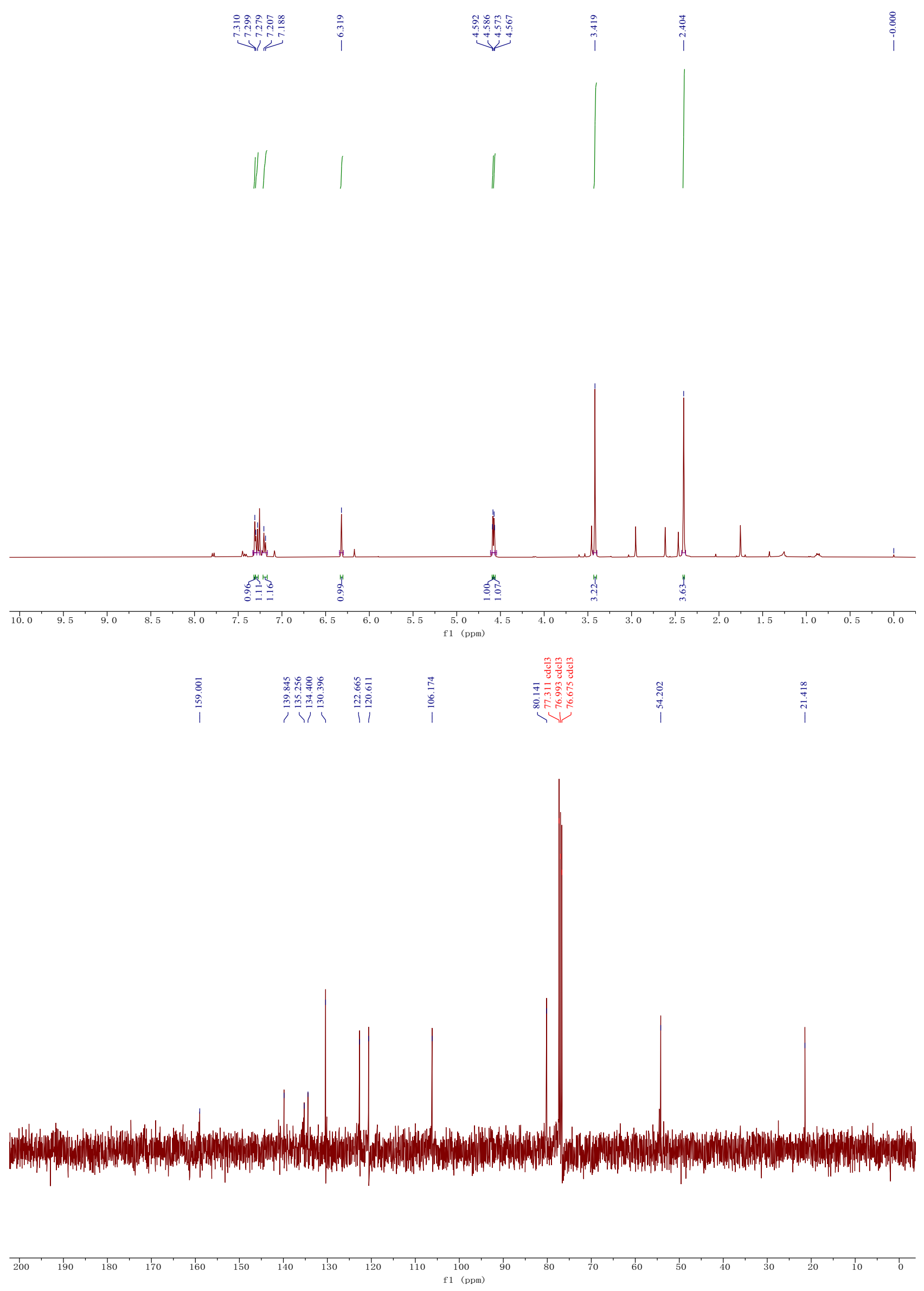

S79 


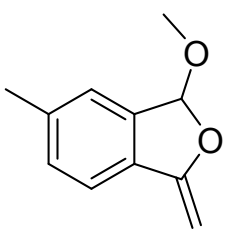

Compound 1c: Yield: $288 \mathrm{mg}, 82 \%$; ${ }^{1} \mathrm{H}$ NMR (400 MHz, $\mathrm{CDCl}_{3}$, TMS) $\delta 7.39(\mathrm{~d}, J=8.5 \mathrm{~Hz}, 1 \mathrm{H})$, $7.23(\mathrm{~s}, 1 \mathrm{H}), 7.17(\mathrm{~d}, J=10.7 \mathrm{~Hz}, 1 \mathrm{H}), 6.30(\mathrm{~s}, 1 \mathrm{H}), 4.55(\mathrm{~d}, J=2.3 \mathrm{~Hz}, 1 \mathrm{H}), 4.54(\mathrm{~d}, J=2.3 \mathrm{~Hz}$, 1H), $3.44(\mathrm{~s}, 3 \mathrm{H}), 2.40(\mathrm{~s}, 3 \mathrm{H})$.

This compound decomposed too fast in neat to get its pure ${ }^{13} C$ NMR spectrum, we had tried several times to just obtain an unclean ${ }^{1} H$ NMR spectrum only. 


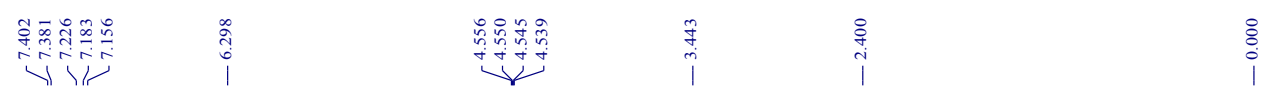

$|\||$

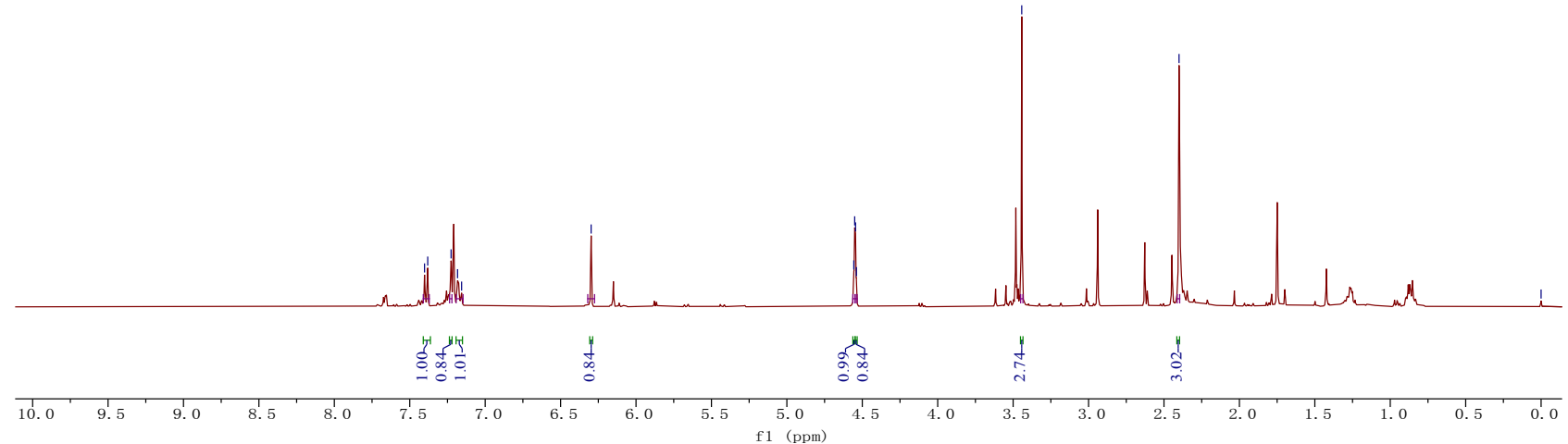




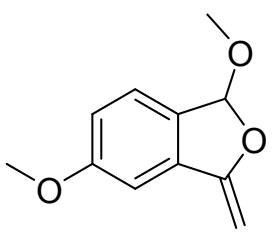

Compound 1d: Yield: $330 \mathrm{mg}, 86 \% ;{ }^{1} \mathrm{H}$ NMR (400 MHz, $\left.\mathrm{CDCl}_{3}, \mathrm{TMS}\right) \delta 7.30(\mathrm{~d}, J=8.1 \mathrm{~Hz}, 1 \mathrm{H})$, $6.97-6.92(\mathrm{~m}, 2 \mathrm{H}), 6.30(\mathrm{~s}, 1 \mathrm{H}), 4.59(\mathrm{~s}, 2 \mathrm{H}), 3.85(\mathrm{~s}, 3 \mathrm{H}), 3.42(\mathrm{~s}, 3 \mathrm{H}) ;{ }^{13} \mathrm{C}\left\{{ }^{1} \mathrm{H}\right\}-\mathrm{NMR}(100 \mathrm{MHz}$, $\left.\mathrm{CDCl}_{3}, \mathrm{TMS}\right) \delta_{\mathrm{C}} 161.2,158.9,135.8,130.4,123.9,116.9,106.1,103.9,80.4,55.5,54.2$. 


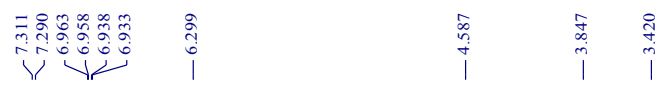
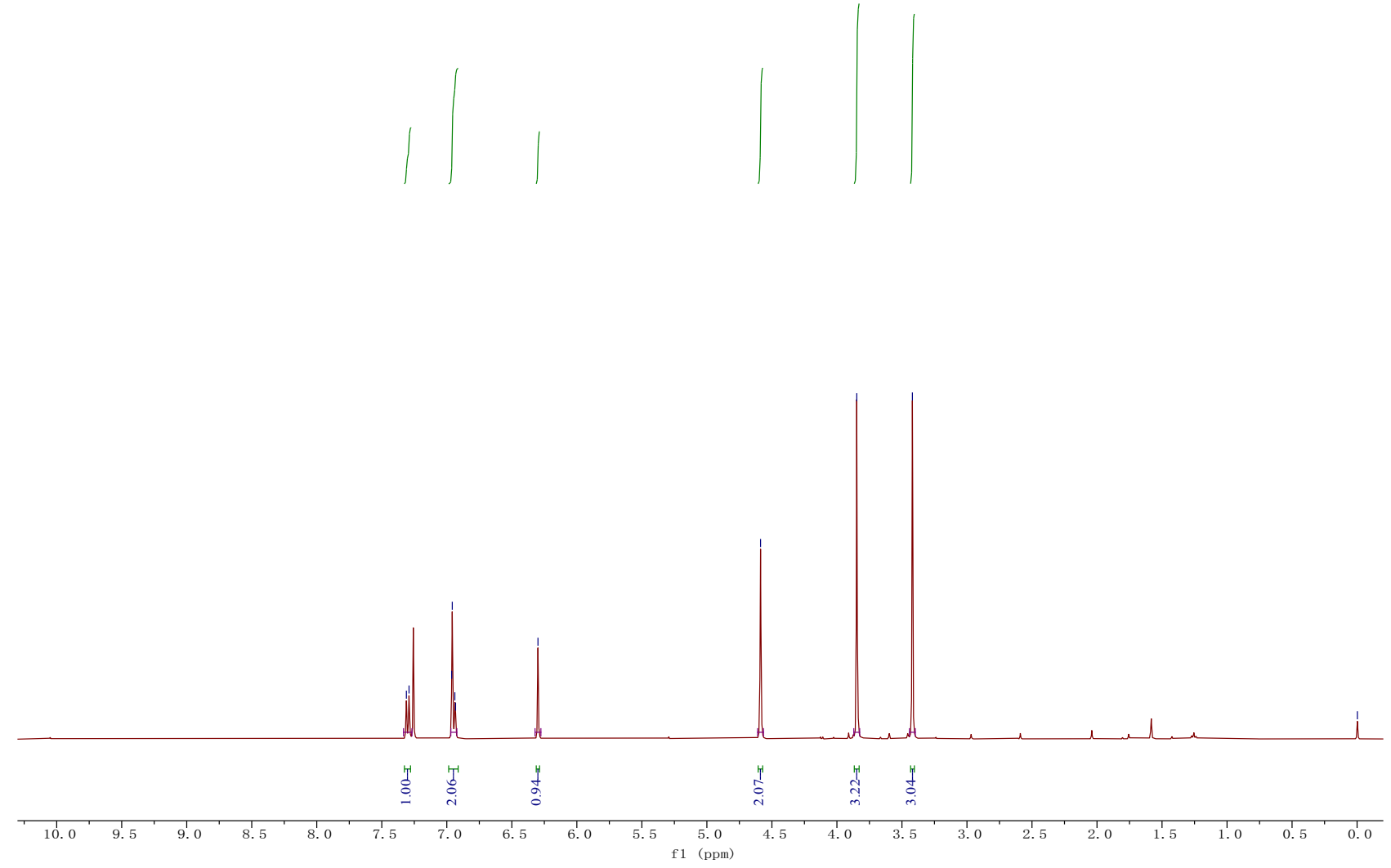

\begin{tabular}{|c|c|c|}
\hline 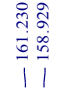 & 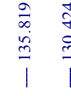 & \\
\hline
\end{tabular}

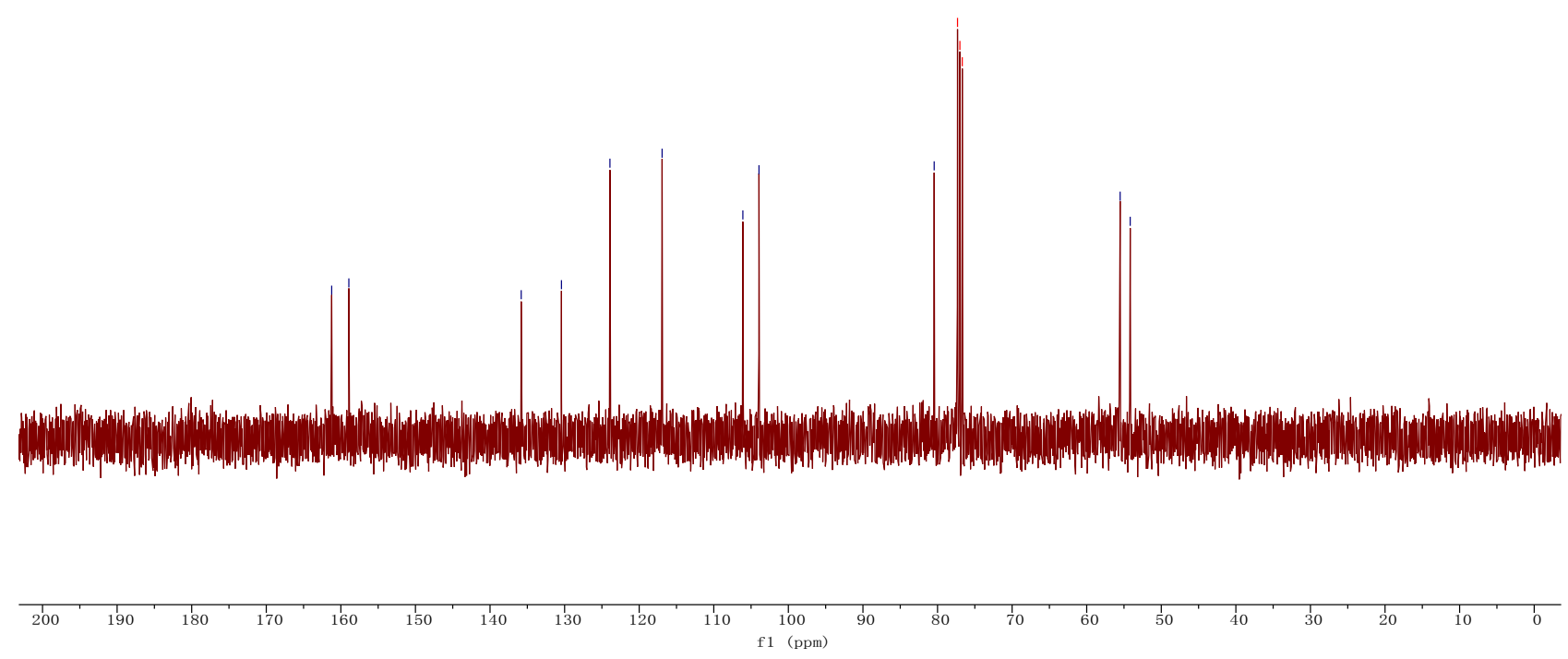


<smiles>C=C1OC(OC)c2cc(Cl)ccc21</smiles>

Compound 1e: Yield: $342 \mathrm{mg}, 87 \%$; ${ }^{1} \mathrm{H}$ NMR (400 MHz, $\mathrm{CDCl}_{3}$, TMS) $\delta 7.46-7.34$ (m, 3H), $6.30(\mathrm{~s}, 1 \mathrm{H}), 4.62(\mathrm{~d}, J=2.5 \mathrm{~Hz}, 1 \mathrm{H}), 4.61(\mathrm{~d}, J=2.6 \mathrm{~Hz}, 1 \mathrm{H}), 3.46(\mathrm{~s}, 3 \mathrm{H}) ;{ }^{13} \mathrm{C}\left\{{ }^{1} \mathrm{H}\right\}-\mathrm{NMR}(100$ $\left.\mathrm{MHz}, \mathrm{CDCl}_{3}, \mathrm{TMS}\right) \delta_{\mathrm{C}} 158.0,139.6,135.2,132.7,130.2,123.4,121.6,105.6,81.4,54.8$. 

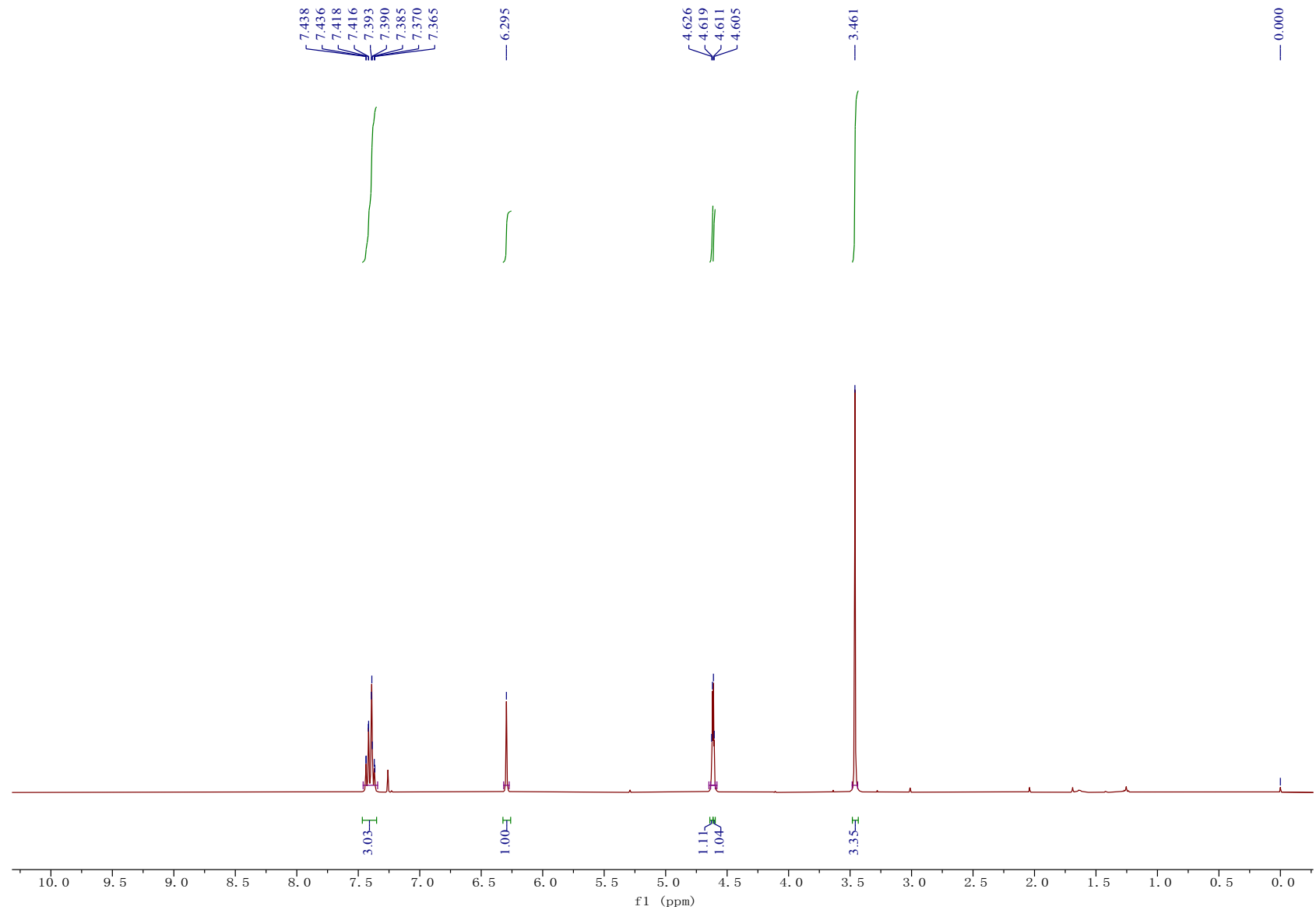

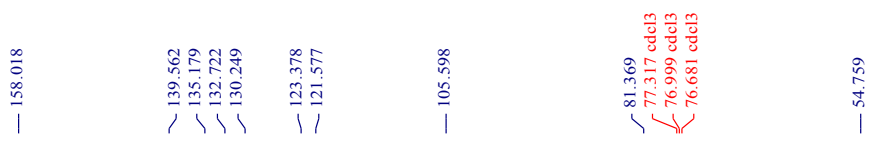

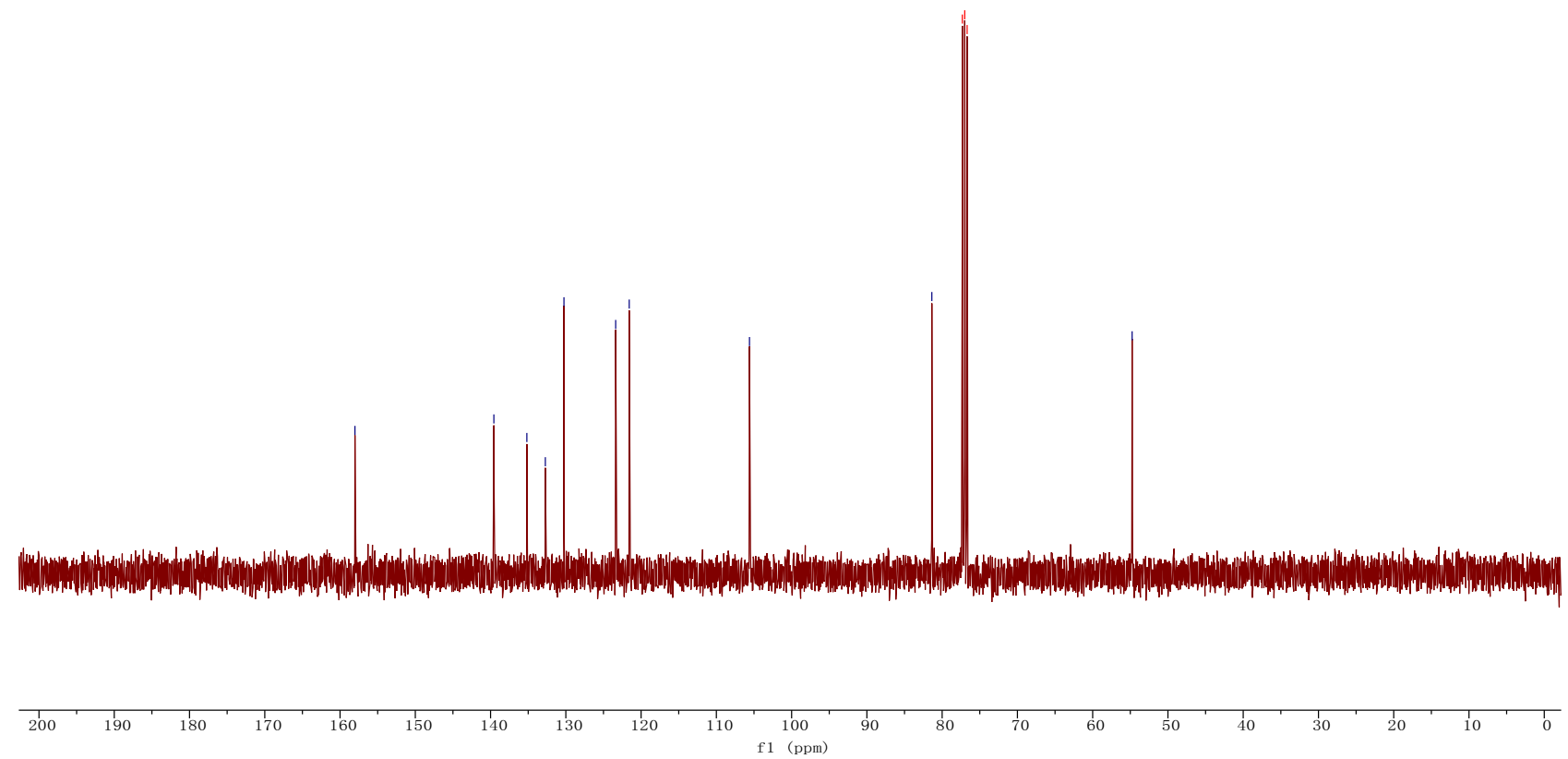


<smiles>C=C1OC(OC)c2cc(F)ccc21</smiles>

Compound 1f: Yield: $309 \mathrm{mg}, 86 \%$; ${ }^{1} \mathrm{H} \mathrm{NMR}\left(400 \mathrm{MHz}, \mathrm{CDCl}_{3}\right.$, TMS) $\delta 7.47$ (dd, $J=8.4,4.7 \mathrm{~Hz}$, 1H), $7.17-7.06(\mathrm{~m}, 2 \mathrm{H}), 6.31(\mathrm{~s}, 1 \mathrm{H}), 4.58(\mathrm{~d}, J=2.6 \mathrm{~Hz}, 1 \mathrm{H}), 4.56(\mathrm{~d}, J=2.3 \mathrm{~Hz}, 1 \mathrm{H}), 3.46(\mathrm{~s}$, $3 \mathrm{H}) ;{ }^{13} \mathrm{C}\left\{{ }^{1} \mathrm{H}\right\}-\mathrm{NMR}\left(100 \mathrm{MHz}, \mathrm{CDCl}_{3}, \mathrm{TMS}\right) \delta_{\mathrm{C}} 163.5(\mathrm{~d}, J=247.1 \mathrm{~Hz}), 158.1,140.0(\mathrm{~d}, J=8.5$ $\mathrm{Hz}), 130.2,122.1(\mathrm{~d}, J=9.2 \mathrm{~Hz}), 117.7(\mathrm{~d}, J=23.8 \mathrm{~Hz}), 110.1(\mathrm{~d}, J=23.8 \mathrm{~Hz}), 105.5(\mathrm{~d}, J=2.9$ $\mathrm{Hz}), 80.4(\mathrm{~d}, J=2.1 \mathrm{~Hz}), 54.7 ;{ }^{19} \mathrm{~F}$ NMR $\left(376 \mathrm{MHz}, \mathrm{CDCl}_{3}\right) \delta-111.3$. 


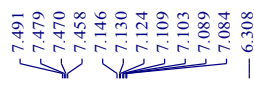

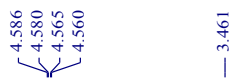
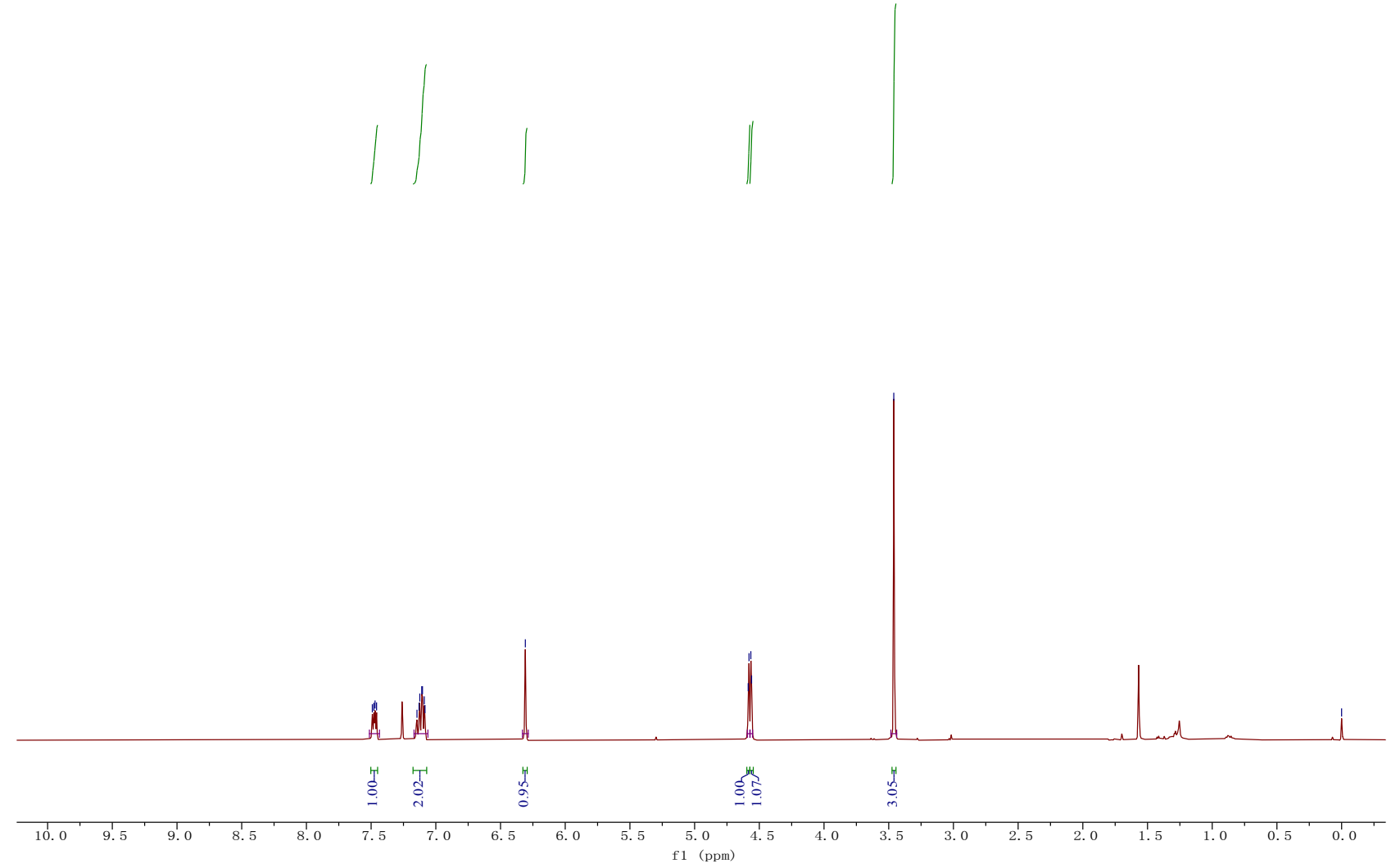

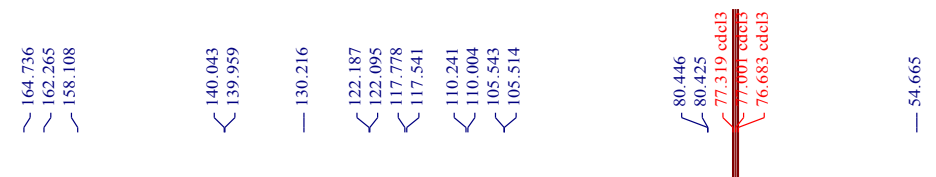
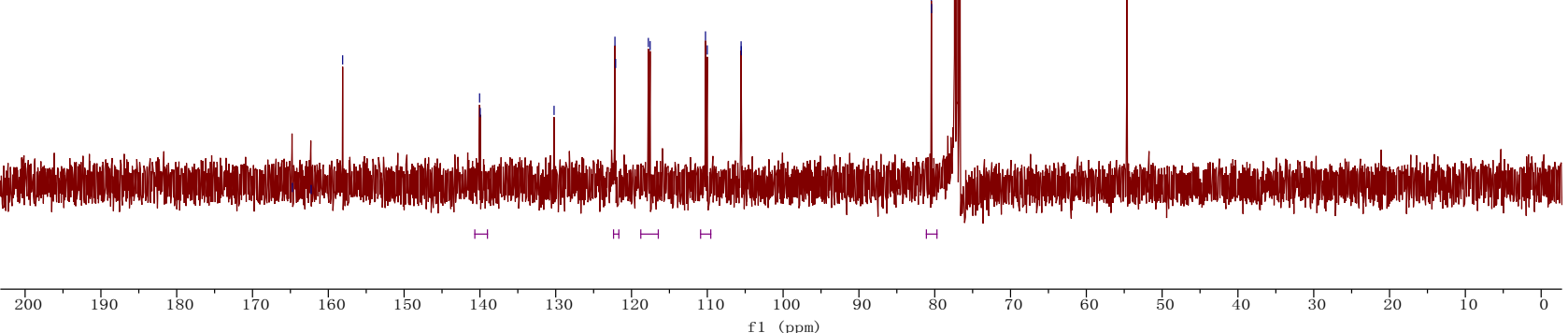


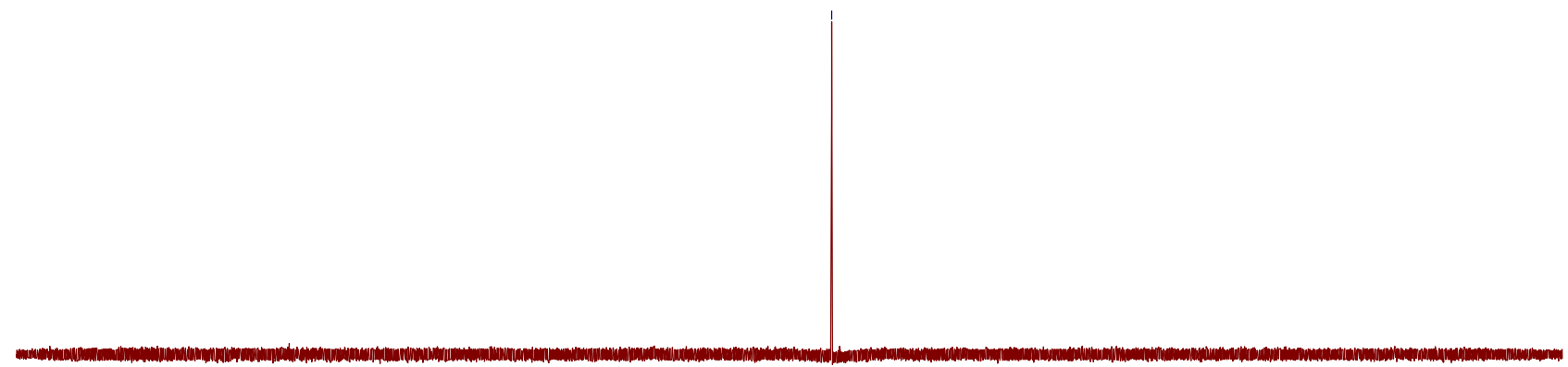

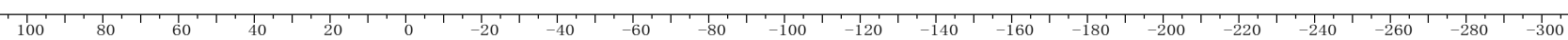
f1 (ppm) 


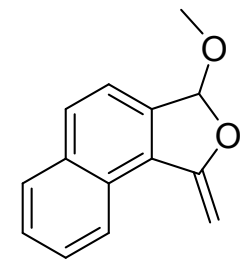

Compound 1g: Yield: $228 \mathrm{mg}, 54 \%$; ${ }^{1} \mathrm{H}$ NMR (400 MHz, $\mathrm{CDCl}_{3}$, TMS) $\delta 8.34-8.29$ (m, 1H), $7.93(\mathrm{~d}, J=8.1 \mathrm{~Hz}, 1 \mathrm{H}), 7.88(\mathrm{~d}, J=8.3 \mathrm{~Hz}, 1 \mathrm{H}), 7.67-7.53(\mathrm{~m}, 2 \mathrm{H}), 7.47(\mathrm{~d}, J=8.3 \mathrm{~Hz}, 1 \mathrm{H})$, $6.42(\mathrm{~s}, 1 \mathrm{H}), 5.09(\mathrm{~d}, J=2.8 \mathrm{~Hz}, 1 \mathrm{H}), 4.97(\mathrm{~d}, J=2.8 \mathrm{~Hz}, 1 \mathrm{H}), 3.44(\mathrm{~s}, 3 \mathrm{H}) ;{ }^{13} \mathrm{C}\left\{{ }^{1} \mathrm{H}\right\}-\mathrm{NMR}(100$ $\left.\mathrm{MHz}, \mathrm{CDCl}_{3}, \mathrm{TMS}\right) \delta_{\mathrm{C}} 159.7,138.0,134.5,131.1,129.3,128.8,127.8,127.3,126.6,123.4,119.8$, 105.2, 85.9, 54.1. 


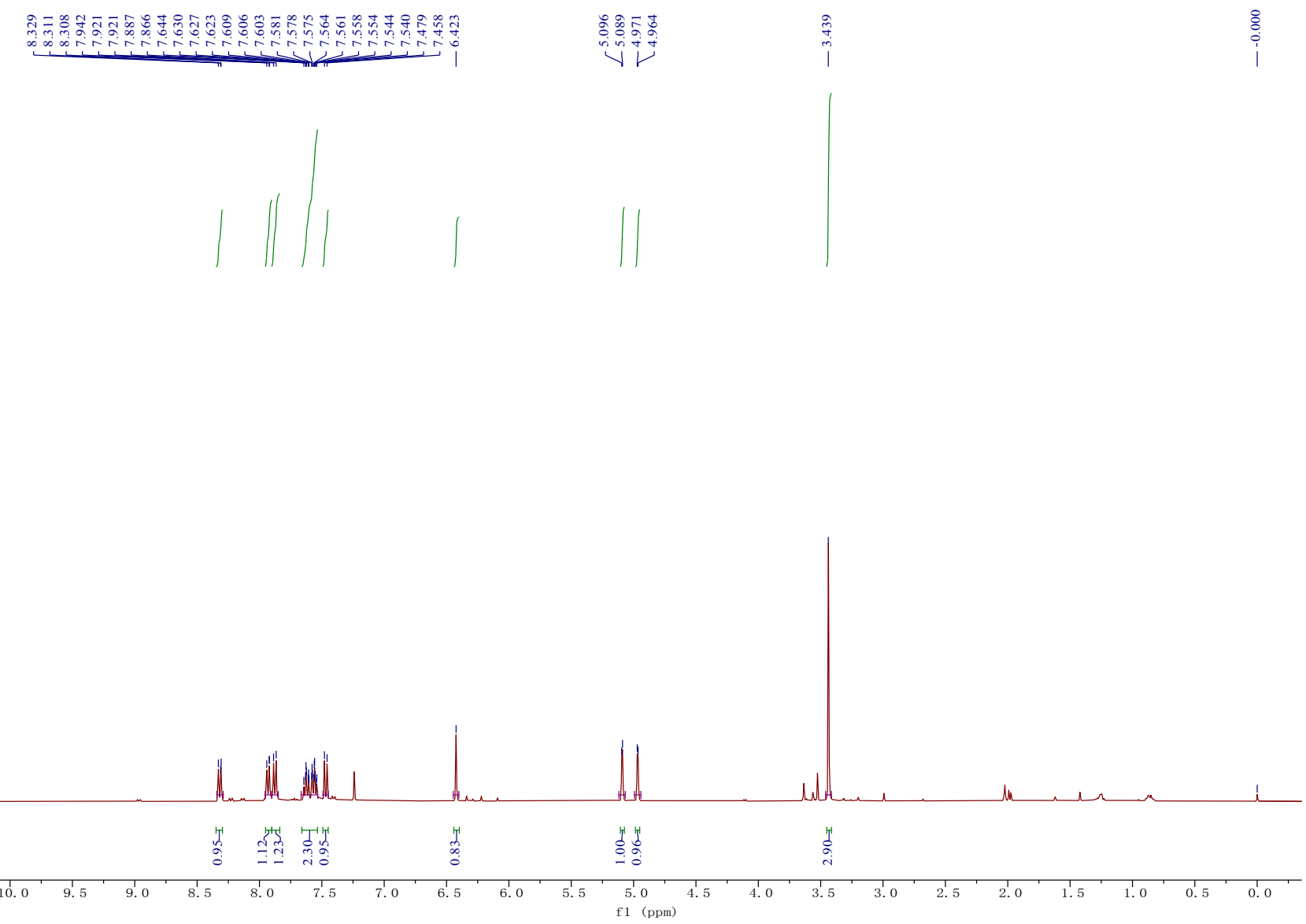

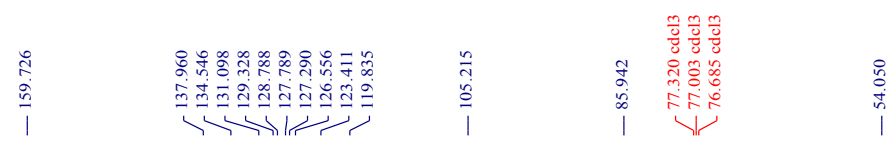

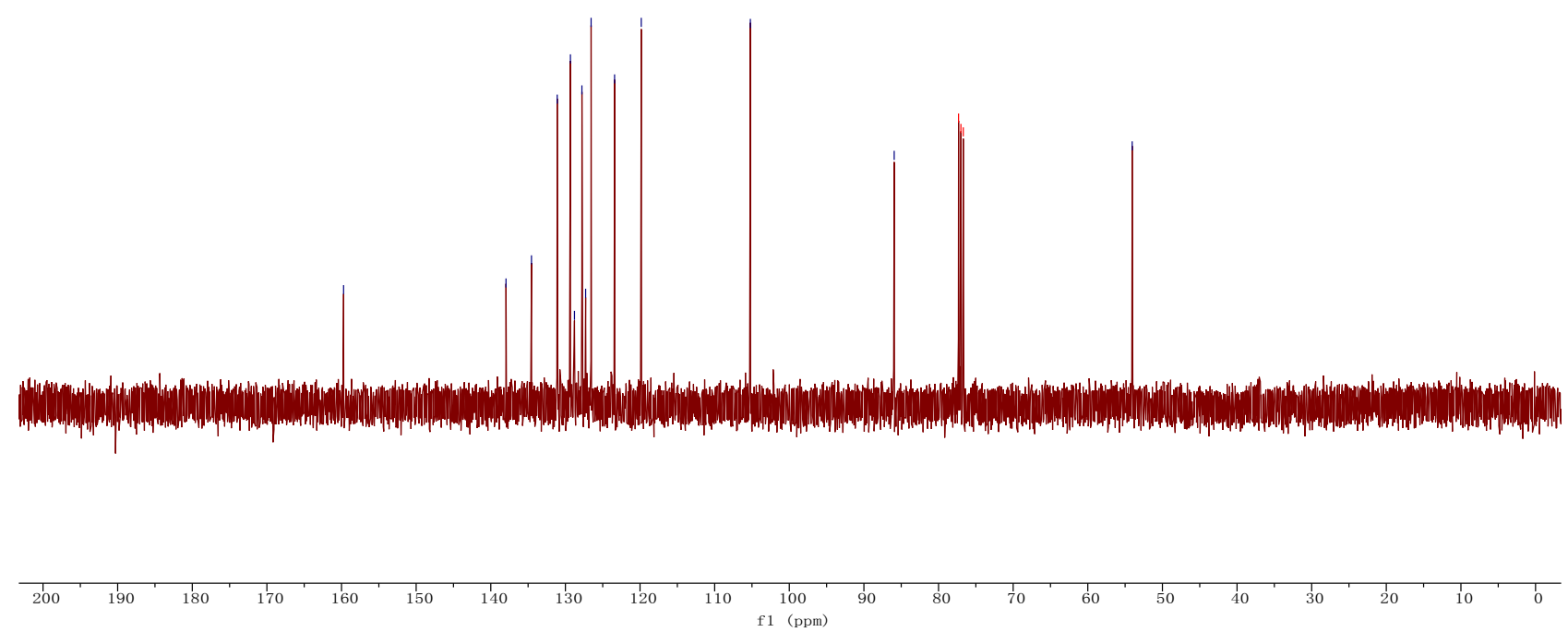




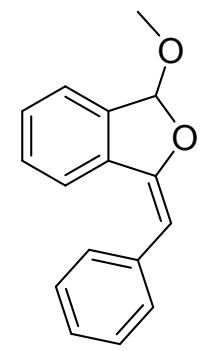

Compound 1h: Yield: $428 \mathrm{mg}$, 90\%; this is a known compound and its spectroscopic data are consistent with those reported ones. ${ }^{1}{ }^{1} \mathrm{H} \mathrm{NMR}\left(400 \mathrm{MHz}, \mathrm{CDCl}_{3}, \mathrm{TMS}\right) \delta 7.77(\mathrm{~d}, J=8.1 \mathrm{~Hz}, 2 \mathrm{H})$, $7.51(\mathrm{~d}, J=8.2 \mathrm{~Hz}, 1 \mathrm{H}), 7.44-7.30(\mathrm{~m}, 5 \mathrm{H}), 7.21-7.13(\mathrm{~m}, 1 \mathrm{H}), 6.51(\mathrm{~s}, 1 \mathrm{H}), 5.97$ (s, 1H), 3.46 $(\mathrm{s}, 3 \mathrm{H}) ;{ }^{13} \mathrm{C}\left\{{ }^{1} \mathrm{H}\right\}-\mathrm{NMR}\left(100 \mathrm{MHz}, \mathrm{CDCl}_{3}, \mathrm{TMS}\right) \delta_{\mathrm{C}} 152.9,136.8,135.7,135.4,129.8,128.9$, $128.32,128.25,125.8,123.0,119.7,107.3,98.1,54.2$. 

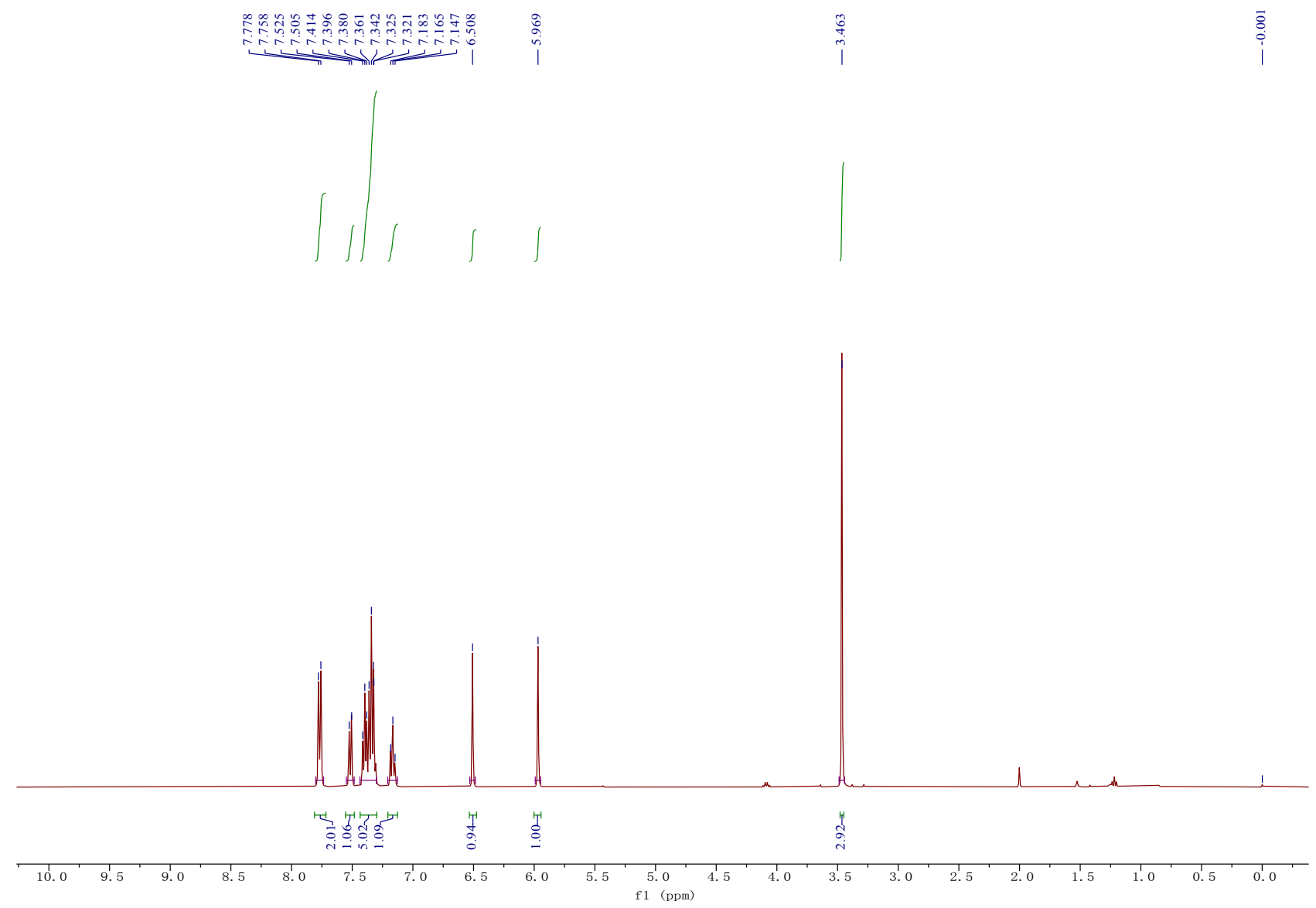

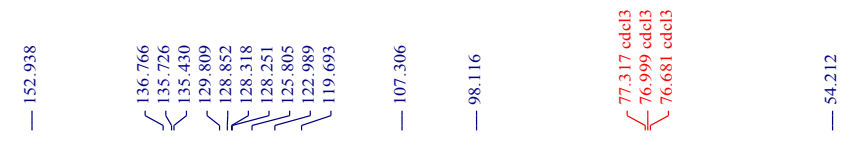

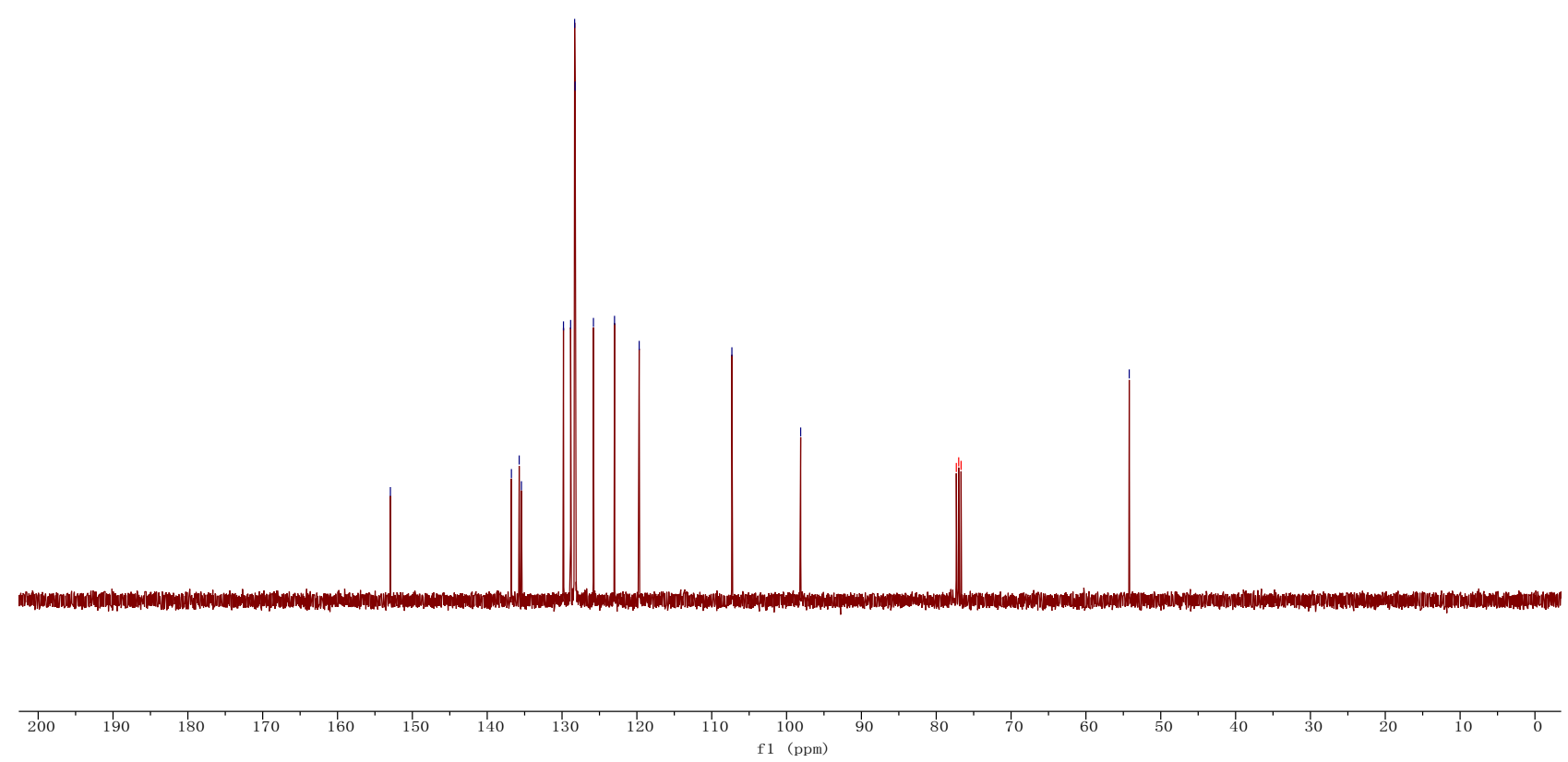




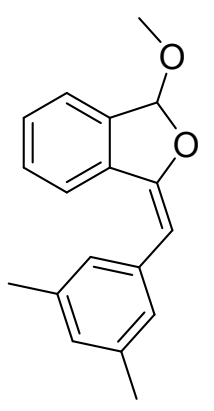

Compound 1i: Yield: $484 \mathrm{mg}, 91 \%$; ${ }^{1} \mathrm{H}$ NMR (400 MHz, $\mathrm{CDCl}_{3}$, TMS) $\delta 7.53$ (dd, J=7.6, $1.2 \mathrm{~Hz}$, 1H), $7.45-7.30(\mathrm{~m}, 5 \mathrm{H}), 6.83(\mathrm{~s}, 1 \mathrm{H}), 6.55(\mathrm{~s}, 1 \mathrm{H}), 5.93(\mathrm{~s}, 1 \mathrm{H}), 3.47(\mathrm{~s}, 3 \mathrm{H}), 2.34(\mathrm{~s}, 6 \mathrm{H})$; ${ }^{13} \mathrm{C}\left\{{ }^{1} \mathrm{H}\right\}$-NMR (100 MHz, $\mathrm{CDCl}_{3}$, TMS) $\delta_{\mathrm{C}} 152.7,137.6,136.7,135.7,135.5,129.8,128.7,127.8$, $126.2,123.0,119.7,107.2,98.3,54.0,21.4$. 

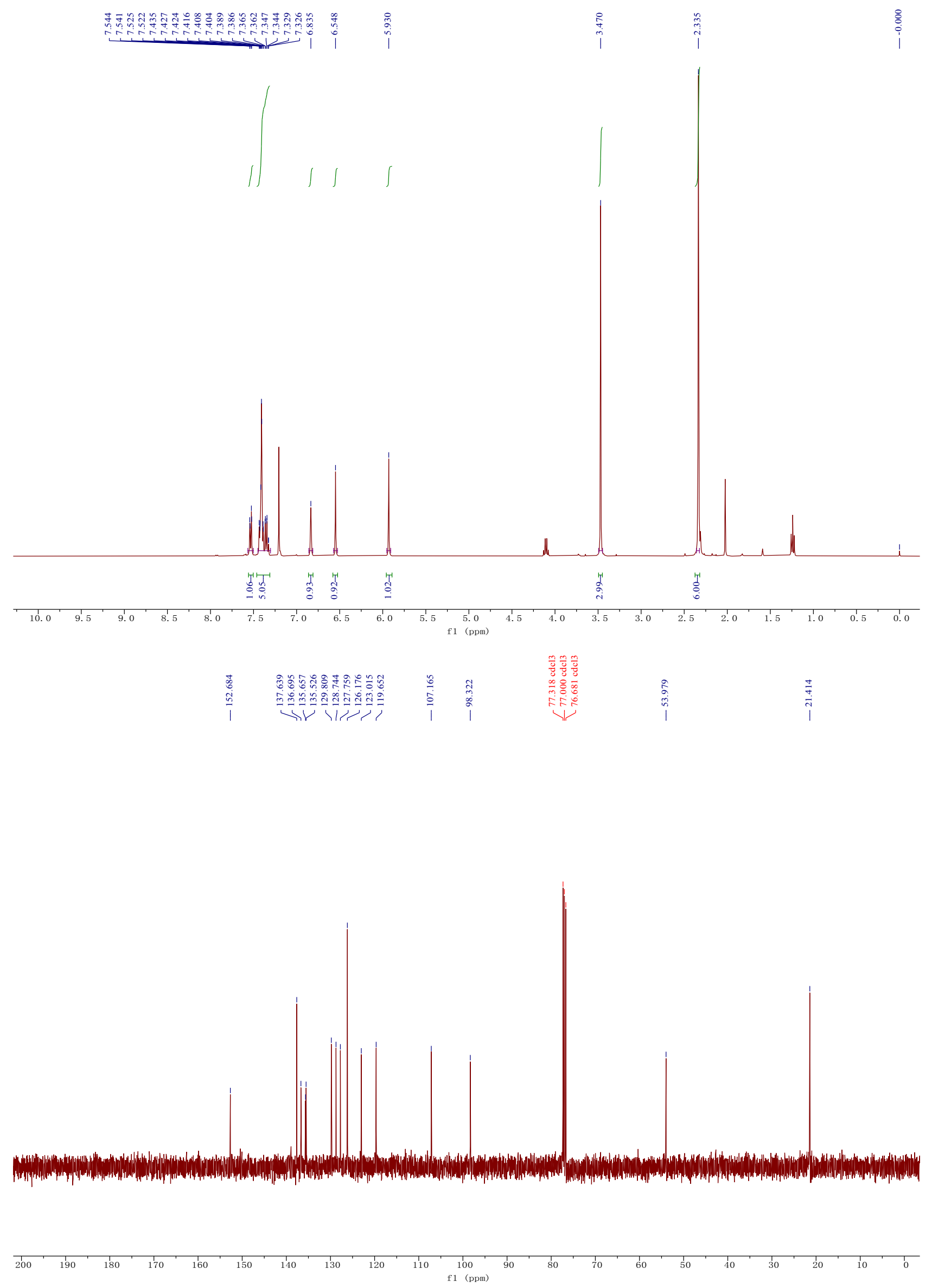


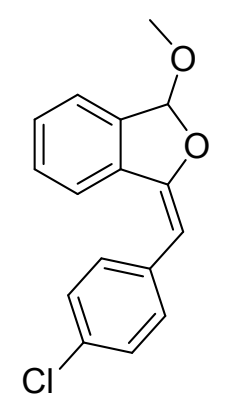

Compound 1j: $500 \mathrm{mg}, 92 \%$; ${ }^{1} \mathrm{H}$ NMR (400 MHz, $\mathrm{CDCl}_{3}$, TMS) $\delta 7.70$ (d, J=8.6 Hz, 2H), $7.60-$ $7.53(\mathrm{~m}, 1 \mathrm{H}), 7.49-7.34(\mathrm{~m}, 3 \mathrm{H}), 7.31$ (d, $J=8.6 \mathrm{~Hz}, 2 \mathrm{H}), 6.55(\mathrm{~s}, 1 \mathrm{H}), 5.94(\mathrm{~s}, 1 \mathrm{H}), 3.50(\mathrm{~s}, 3 \mathrm{H})$; ${ }^{13} \mathrm{C}\left\{{ }^{1} \mathrm{H}\right\}$-NMR (100 MHz, $\mathrm{CDCl}_{3}$, TMS) $\delta_{\mathrm{C}} 153.4,136.9,135.2,134.3,131.2,130.0,129.4,129.2$, 128.5, 123.1, 119.8, 107.5, 97.0, 54.4. 

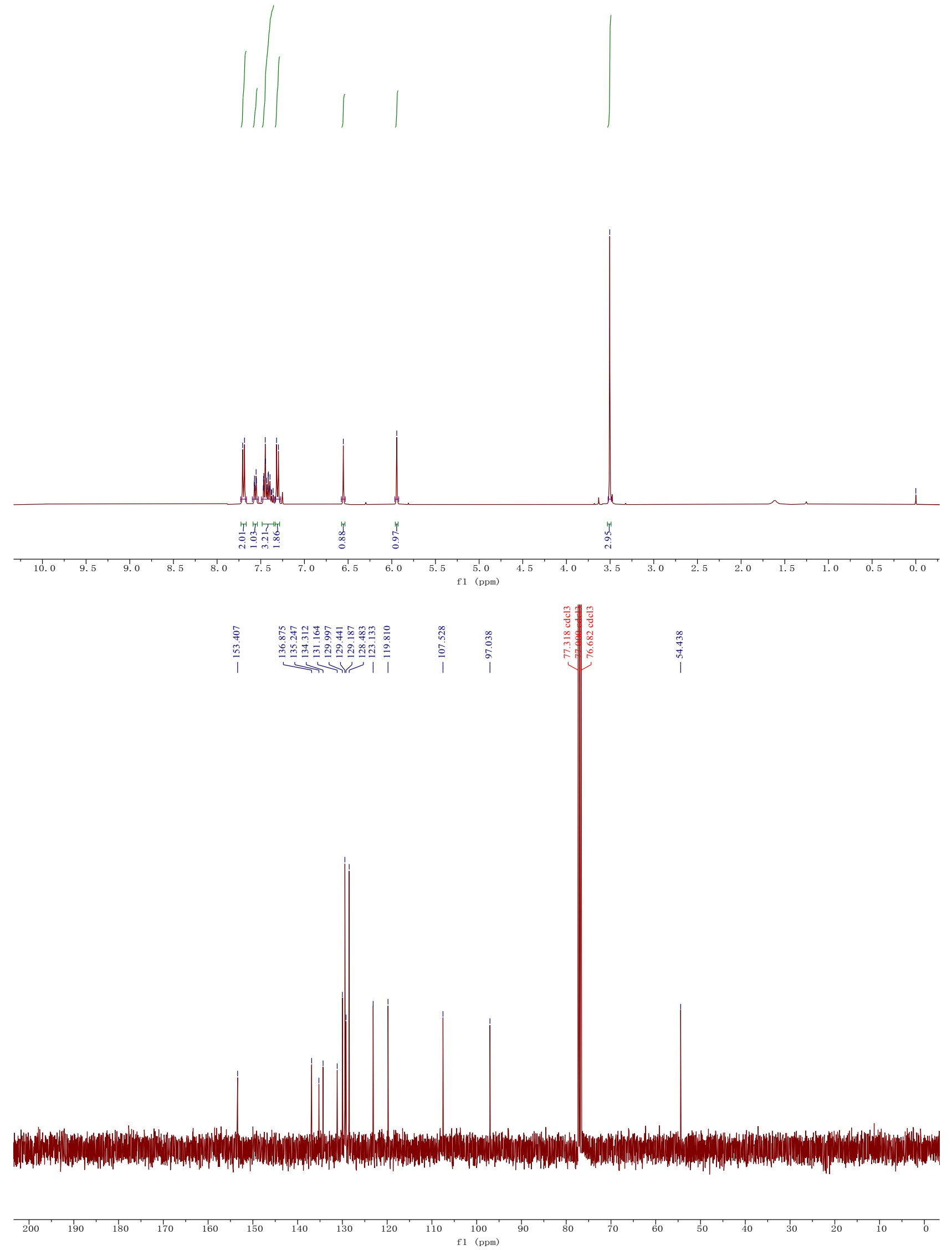


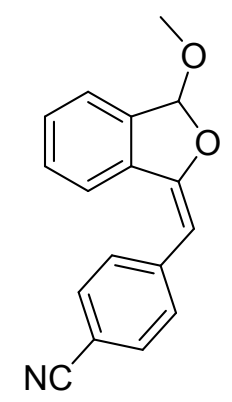

Compound 1k: $478 \mathrm{mg}, 91 \%$; ${ }^{1} \mathrm{H}$ NMR (400 MHz, $\left.\mathrm{CDCl}_{3}, \mathrm{TMS}\right) \delta 7.83$ (dd, $J=8.5,1.8 \mathrm{~Hz}, 2 \mathrm{H}$ ), $7.64-7.58(\mathrm{~m}, 3 \mathrm{H}), 7.51-7.41(\mathrm{~m}, 3 \mathrm{H}), 6.59(\mathrm{~d}, J=1.6 \mathrm{~Hz}, 1 \mathrm{H}), 5.99$ (d, $J=1.7 \mathrm{~Hz}, 1 \mathrm{H}), 3.54(\mathrm{~s}$, $3 \mathrm{H}) ;{ }^{13} \mathrm{C}\left\{{ }^{1} \mathrm{H}\right\}-\mathrm{NMR}\left(100 \mathrm{MHz}, \mathrm{CDCl}_{3}\right.$, TMS $) \delta_{\mathrm{C}} 155.8,140.7,137.3,134.8,132.1,130.2,129.9$, $128.4,123.2,120.2,119.6,108.3,108.1,96.7,54.8$ 

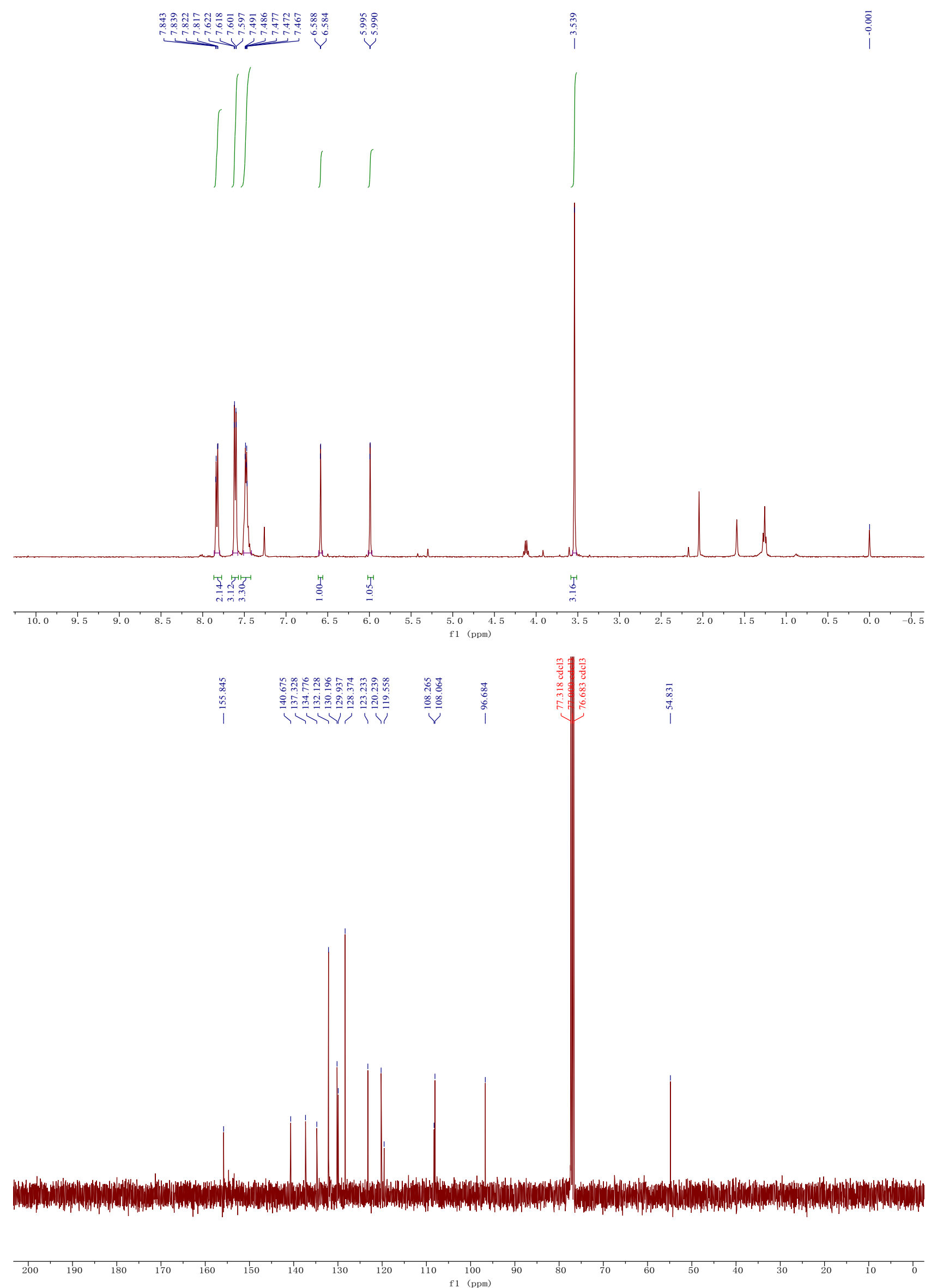


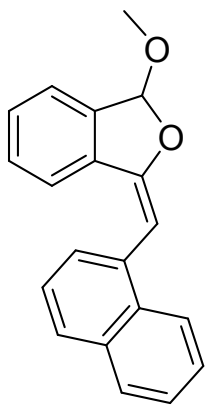

Compound 1I: $512 \mathrm{mg}, 89 \%$; ${ }^{1} \mathrm{H} \mathrm{NMR}\left(400 \mathrm{MHz}, \mathrm{CDCl}_{3}\right.$, TMS) $\delta 8.33$ (dd, $J=7.4,1.2 \mathrm{~Hz}, 1 \mathrm{H}$ ), $8.25(\mathrm{~d}, J=8.3 \mathrm{~Hz}, 1 \mathrm{H}), 7.85(\mathrm{dd}, J=7.9,1.6 \mathrm{~Hz}, 1 \mathrm{H}), 7.72(\mathrm{~d}, J=7.8 \mathrm{~Hz}, 2 \mathrm{H}), 7.56-7.38(\mathrm{~m}$, $6 \mathrm{H}), 6.72(\mathrm{~s}, 1 \mathrm{H}), 6.56(\mathrm{~s}, 1 \mathrm{H}), 3.50(\mathrm{~s}, 3 \mathrm{H}) ;{ }^{13} \mathrm{C}\left\{{ }^{1} \mathrm{H}\right\}-\mathrm{NMR}\left(100 \mathrm{MHz}, \mathrm{CDCl}_{3}, \mathrm{TMS}\right) \delta_{\mathrm{C}} 153.8$, 137.0, 135.6, 133.8, 131.5, 131.2, 130.0, 129.1, 128.7, 126.6, 126.5, 125.7, 125.7, 125.4, 123.7, $123.1,119.9,107.4,93.6,54.5$. 

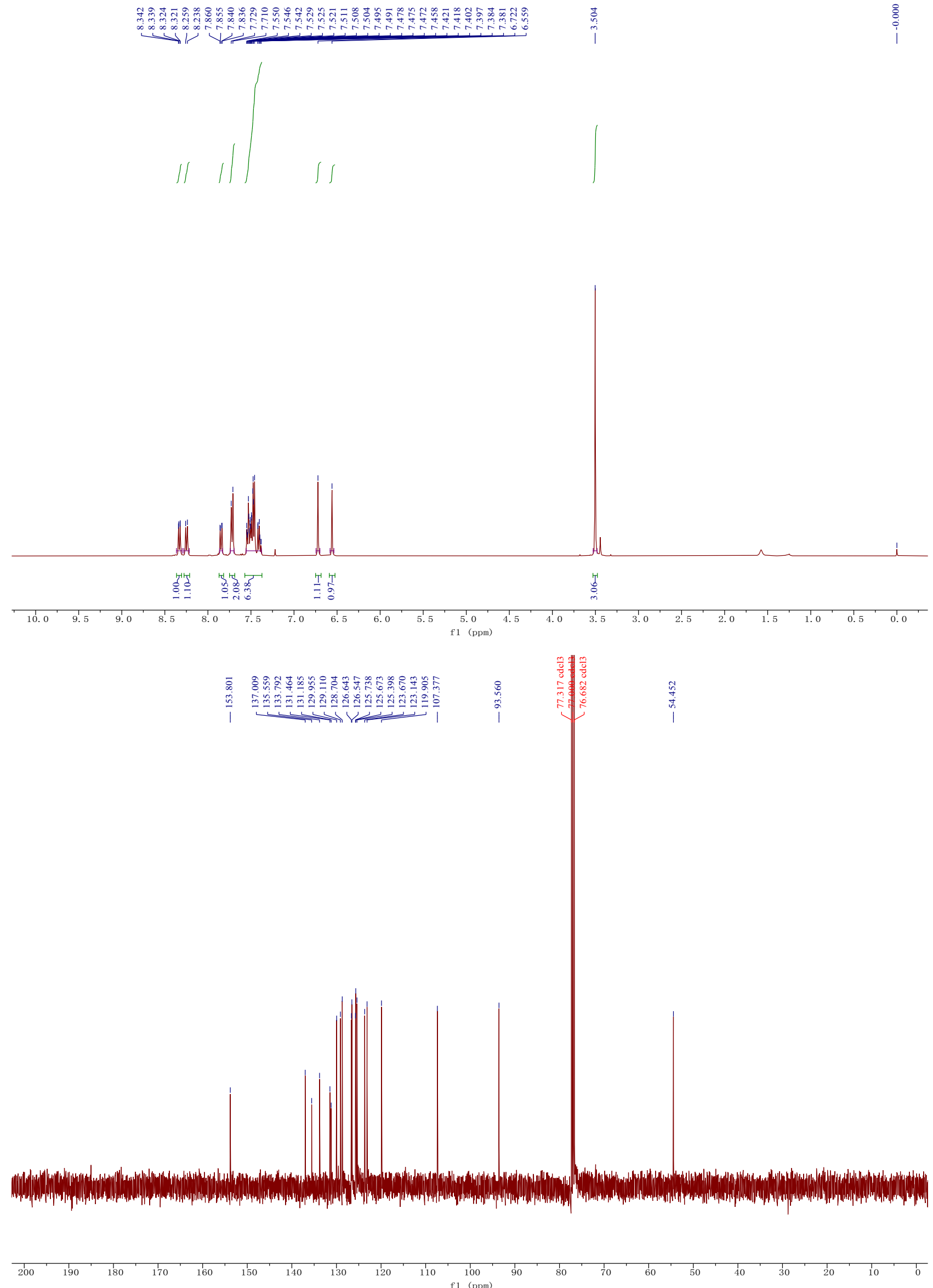


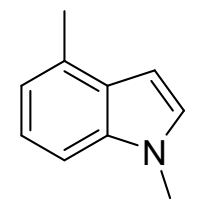

Compound 2b: $267 \mathrm{mg}, 92 \%$; this is a known compound and its spectroscopic data are consistent with those reported ones. ${ }^{2}{ }^{1} \mathrm{H}$ NMR $\left(400 \mathrm{MHz} \mathrm{CDCl}_{3}, \mathrm{TMS}\right) \delta 7.12(\mathrm{~s}, 1 \mathrm{H}), 7.11(\mathrm{~d}, J=1.4 \mathrm{~Hz}$, 1H), $6.97(\mathrm{~d}, J=3.1 \mathrm{~Hz}, 1 \mathrm{H}), 6.91-6.87(\mathrm{~m}, 1 \mathrm{H}), 6.47$ (d, $J=3.1 \mathrm{~Hz}, 1 \mathrm{H}), 3.68(\mathrm{~s}, 3 \mathrm{H}), 2.54$ (s, $1 \mathrm{H}) ;{ }^{13} \mathrm{C}\left\{{ }^{1} \mathrm{H}\right\}-\mathrm{NMR}\left(100 \mathrm{MHz}, \mathrm{CDCl}_{3}, \mathrm{TMS}\right) \delta_{\mathrm{C}} 136.3,130.2,128.3,128.0,121.6,119.4,106.7$, $99.3,32.8,18.6$. 


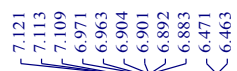

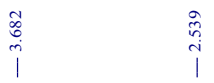

8
$\vdots$
0
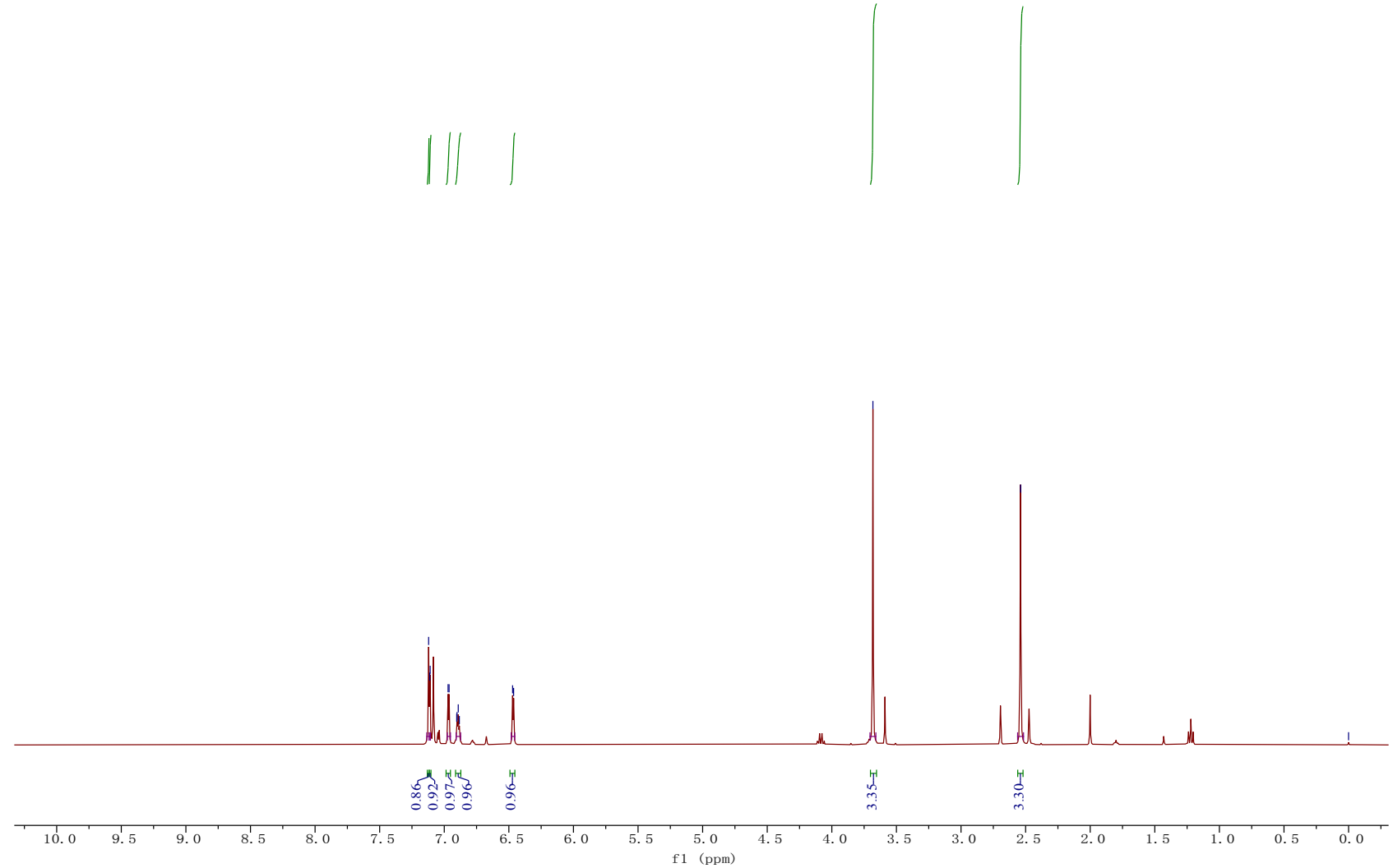

\begin{tabular}{|c|c|c|c|}
\hline 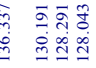 & 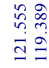 & 6 & $\begin{array}{l}\text { \&े } \\
\stackrel{3}{g}\end{array}$ \\
\hline 1$\rangle$ & 11 & | & 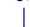 \\
\hline
\end{tabular}
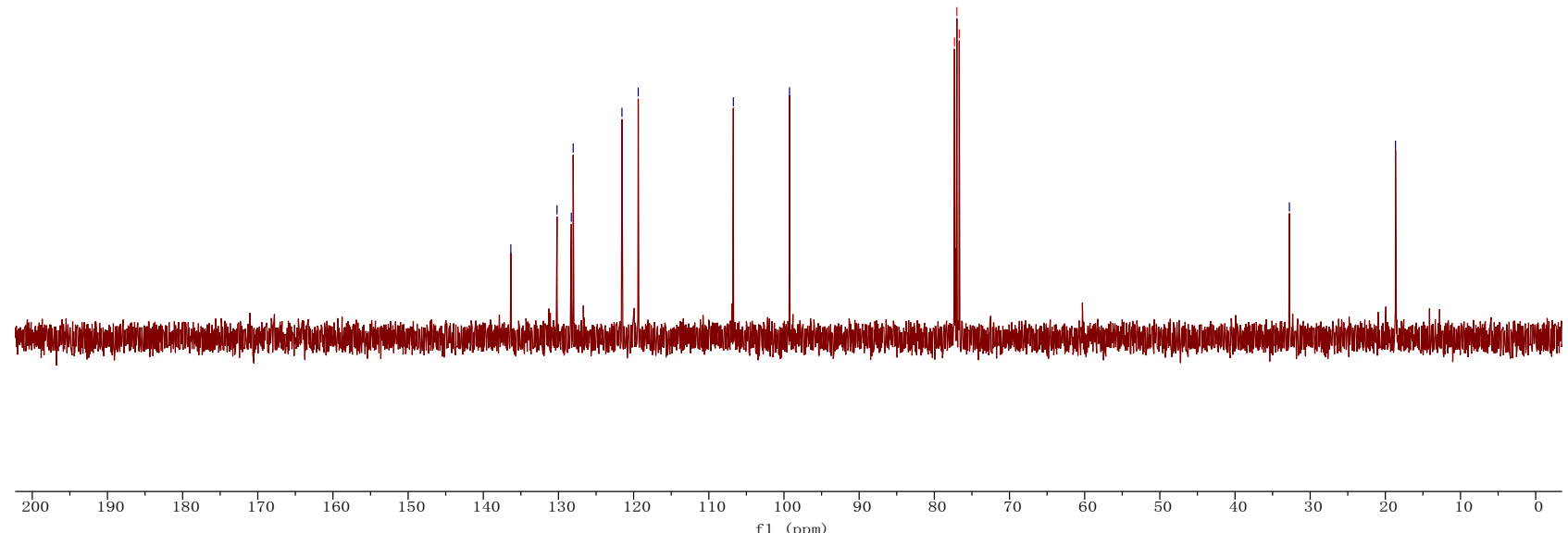


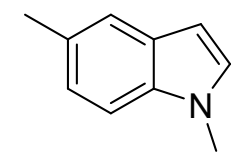

Compound 2c: $261 \mathrm{mg}, 90 \%$; this is a known compound and its spectroscopic data are consistent with those reported ones. ${ }^{2}{ }^{1} \mathrm{H}$ NMR (400 MHz, $\mathrm{CDCl}_{3}$, TMS) $\delta 7.39$ (s, 1H), 7.17 (dd, $J=8.3,1.9$ $\mathrm{Hz}, 1 \mathrm{H}), 7.03(\mathrm{dd}, J=8.3,1.9 \mathrm{~Hz}, 1 \mathrm{H}), 6.95(\mathrm{dd}, J=3.1,1.6 \mathrm{~Hz}, 1 \mathrm{H}), 6.37$ (t, $J=2.8 \mathrm{~Hz}, 1 \mathrm{H}), 3.68$ (s, 3H), $2.44(\mathrm{~s}, 3 \mathrm{H}) ;{ }^{13} \mathrm{C}\left\{{ }^{1} \mathrm{H}\right\}-\mathrm{NMR}\left(100 \mathrm{MHz}, \mathrm{CDCl}_{3}, \mathrm{TMS}\right) \delta_{\mathrm{C}} 135.1,128.8,128.7,128.3,123.0$, 120.4, 108.8, 100.2, 32.7, 21.4 . 

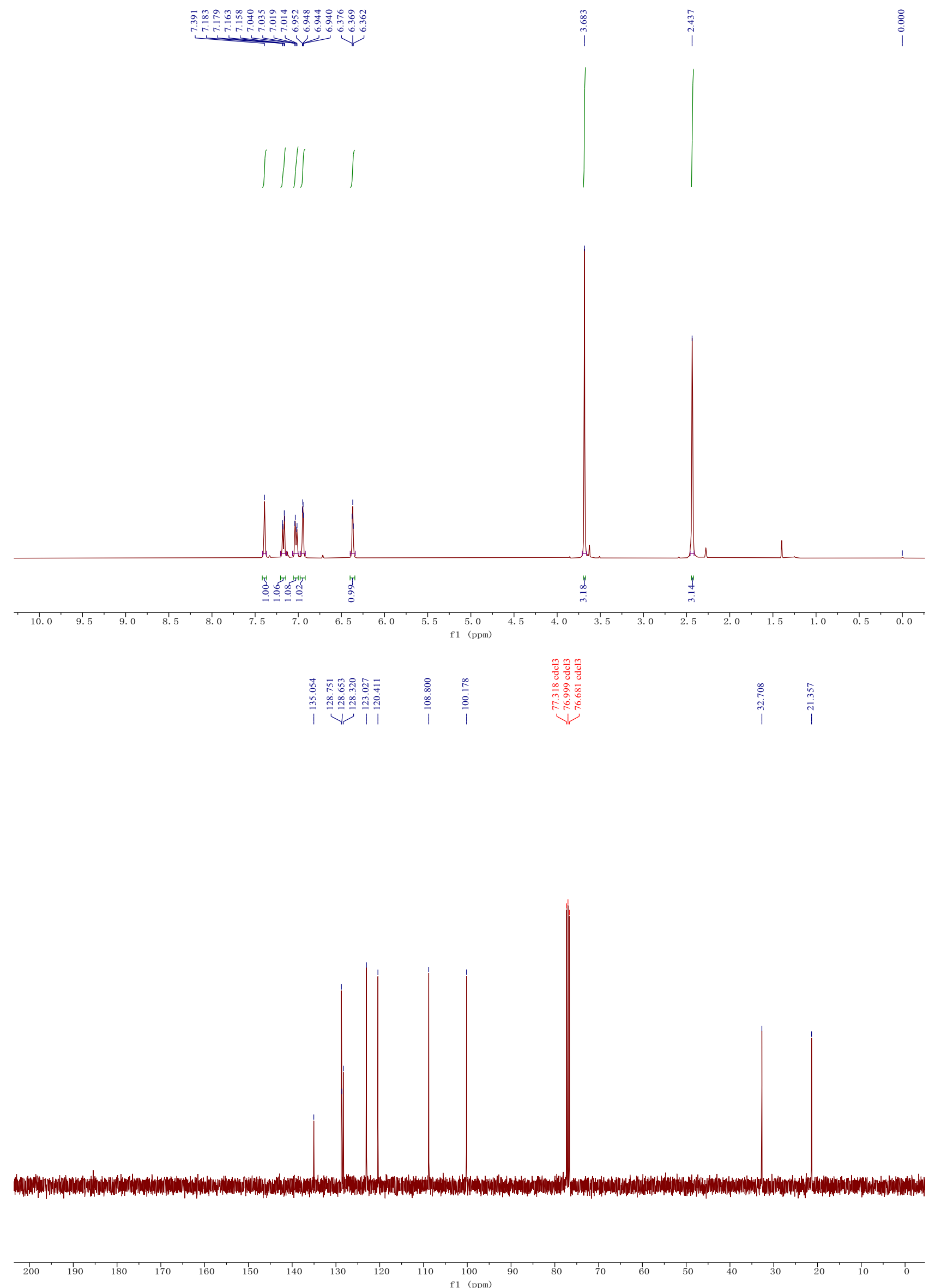


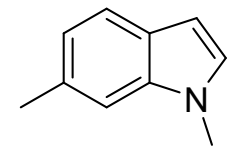

Compound 2d: $267 \mathrm{mg}, 92 \%$; this is a known compound and its spectroscopic data are consistent with those reported ones. ${ }^{2}{ }^{1} \mathrm{H}$ NMR (400 MHz, $\left.\mathrm{CDCl}_{3}, \mathrm{TMS}\right) \delta 7.50$ (d, $\left.J=8.0 \mathrm{~Hz}, 1 \mathrm{H}\right), 7.11$ (s, $1 \mathrm{H}), 6.98-6.90(\mathrm{~m}, 2 \mathrm{H}), 6.42(\mathrm{dd}, J=3.2,0.9 \mathrm{~Hz}, 1 \mathrm{H}), 3.73(\mathrm{~s}, 3 \mathrm{H}), 2.49(\mathrm{~s}, 3 \mathrm{H}) ;{ }^{13} \mathrm{C}\left\{{ }^{1} \mathrm{H}\right\}-\mathrm{NMR}$ $\left(100 \mathrm{MHz}, \mathrm{CDCl}_{3}, \mathrm{TMS}\right) \delta_{\mathrm{C}} 137.0,131.2,128.2,126.2,121.0,120.4,109.1,100.6,32.7,21.9$. 


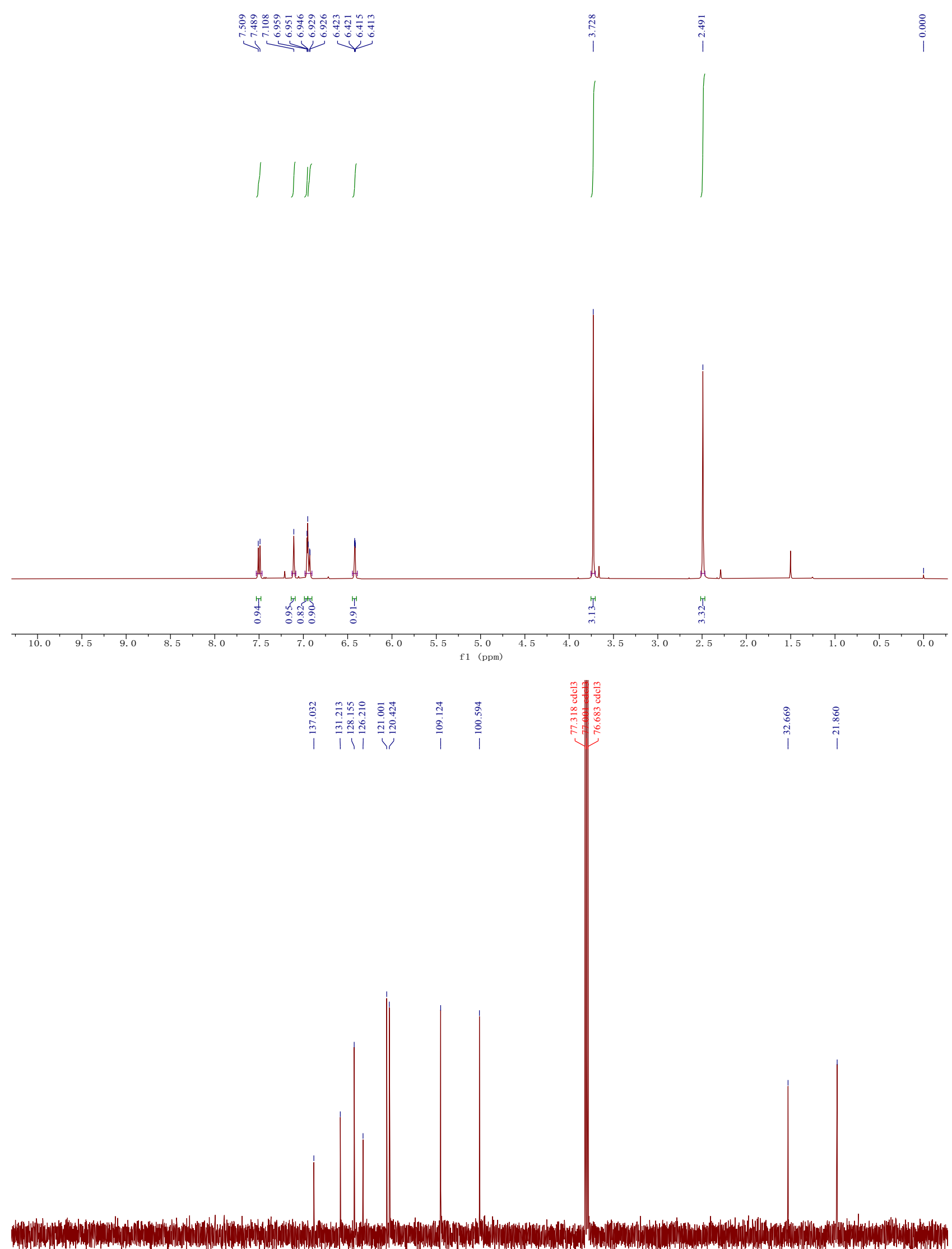

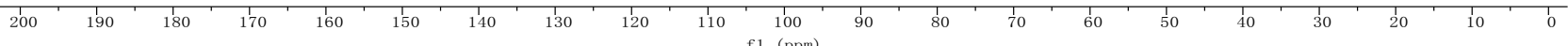




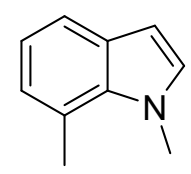

Compound 2e: $258 \mathrm{mg}, 89 \%$; this is a known compound and its spectroscopic data are consistent with those reported ones. ${ }^{2}{ }^{1} \mathrm{H}$ NMR (400 $\mathrm{MHz}, \mathrm{CDCl}_{3}$, TMS) $\delta 7.46-7.39(\mathrm{~m}, 1 \mathrm{H}), 7.11(\mathrm{~s}, 1 \mathrm{H})$, $6.93(\mathrm{td}, J=5.6,5.2,2.7 \mathrm{~Hz}, 1 \mathrm{H}), 6.87(\mathrm{dd}, J=7.0,3.3 \mathrm{~Hz}, 2 \mathrm{H}), 6.39(\mathrm{td}, J=2.9,1.4 \mathrm{~Hz}, 1 \mathrm{H}), 3.96$ (s, 3H), $2.71(\mathrm{~s}, 3 \mathrm{H}) ;{ }^{13} \mathrm{C}\left\{{ }^{1} \mathrm{H}\right\}-\mathrm{NMR}\left(100 \mathrm{MHz}, \mathrm{CDCl}_{3}\right.$, TMS $) \delta_{\mathrm{C}} 135.3,130.3,129.5,124.0,121.1$, $119.5,119.0,100.8,36.6,19.6$. 

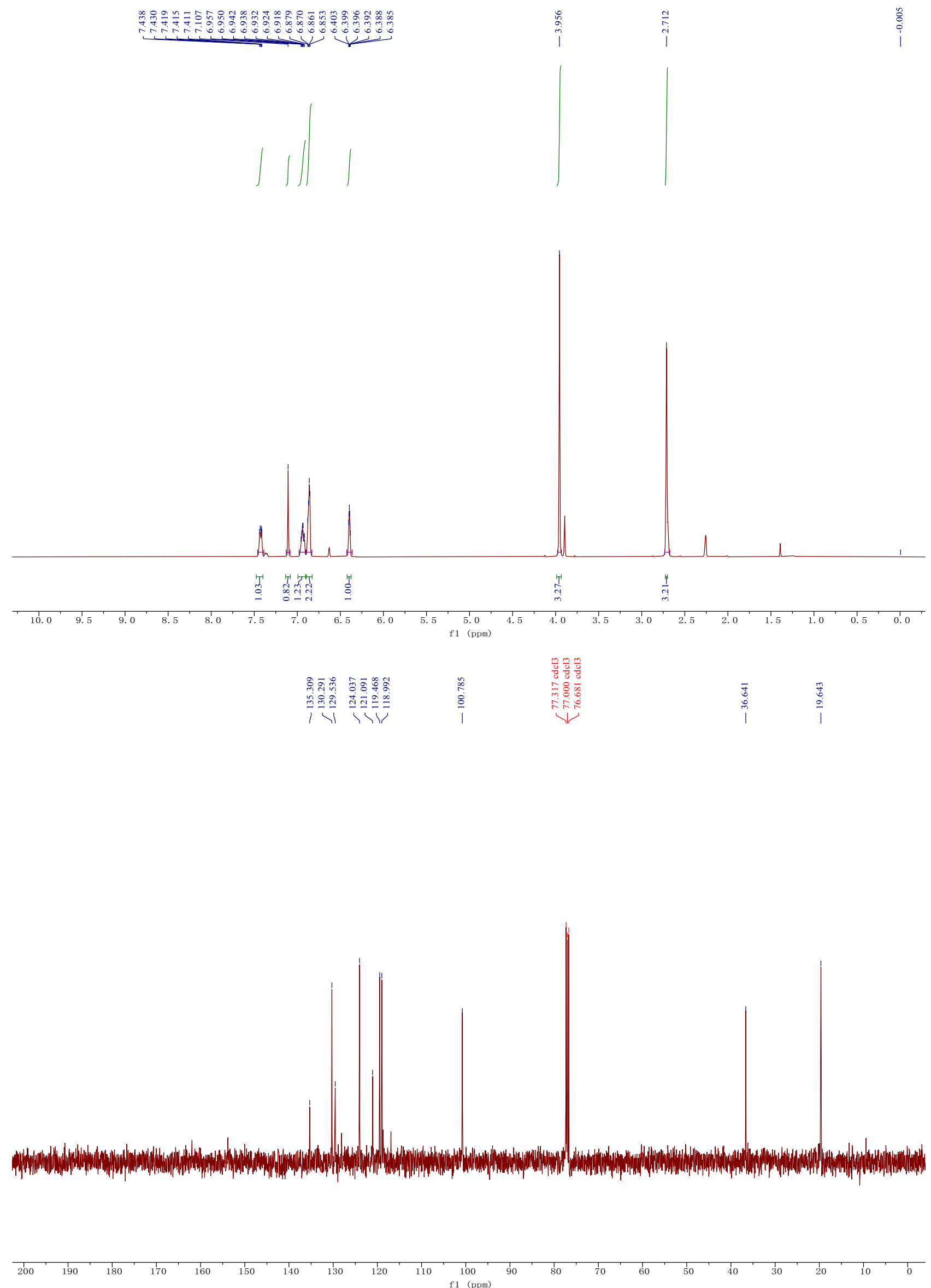
<smiles>Cn1ccc2cc(Cl)ccc21</smiles>

Compound 2f: $297 \mathrm{mg}$, 90\%; this is a known compound and its spectroscopic data are consistent with those reported ones. ${ }^{3}{ }^{1} \mathrm{H}$ NMR (400 $\mathrm{MHz}, \mathrm{CDCl}_{3}$, TMS) $\delta 7.57-7.52(\mathrm{~m}, 1 \mathrm{H}), 7.13(\mathrm{~s}, 1 \mathrm{H})$, $7.12(\mathrm{~s}, 1 \mathrm{H}), 6.98(\mathrm{~d}, J=3.1 \mathrm{~Hz}, 1 \mathrm{H}), 6.39-6.35(\mathrm{~m}, 1 \mathrm{H}), 3.66(\mathrm{~s}, 3 \mathrm{H}) ;{ }^{13} \mathrm{C}\left\{{ }^{1} \mathrm{H}\right\}-\mathrm{NMR}(100 \mathrm{MHz}$, $\left.\mathrm{CDCl}_{3}, \mathrm{TMS}\right) \delta_{\mathrm{C}} 135.0,130.1,129.3,124.9,121.5,120.0,110.1,100.4,32.7$. 


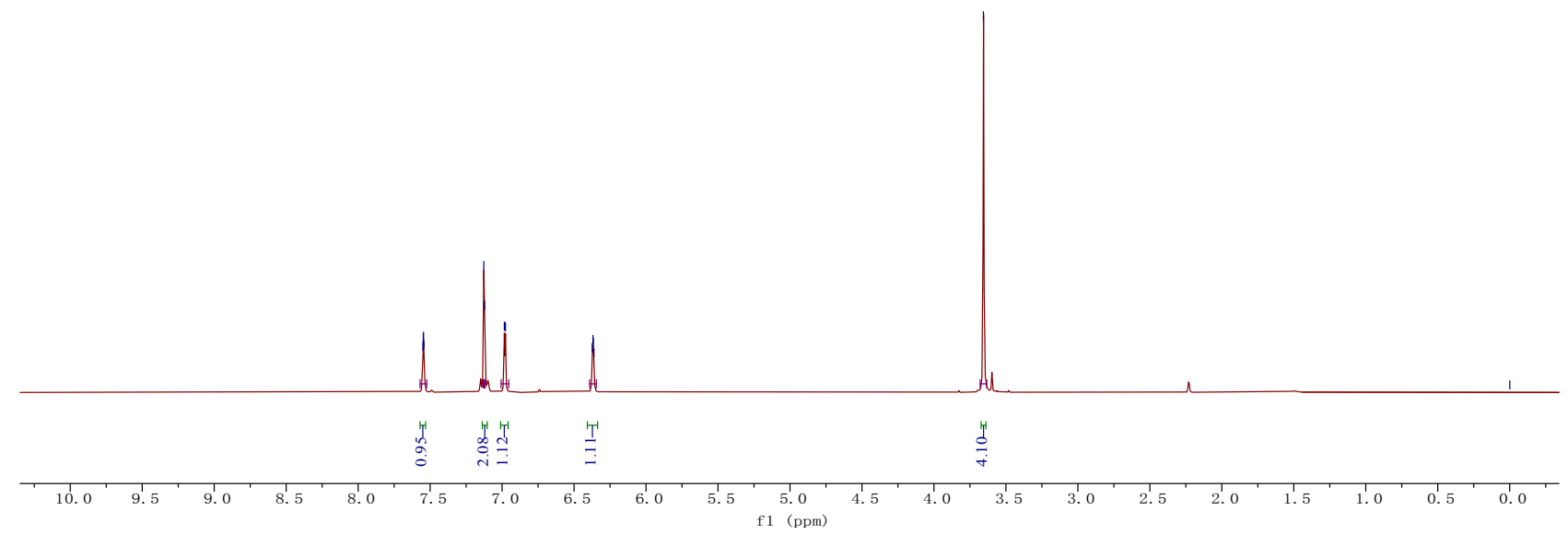

\begin{tabular}{|c|c|c|c|}
\hline 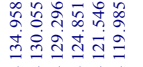 & 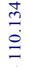 & $\begin{array}{l}\bar{o} \\
\dot{q} \\
\stackrel{8}{0}\end{array}$ & 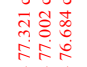 \\
\hline, $11<>1$ & $\overrightarrow{\mid}$ & $\vec{v}$ & 4 \\
\hline
\end{tabular}

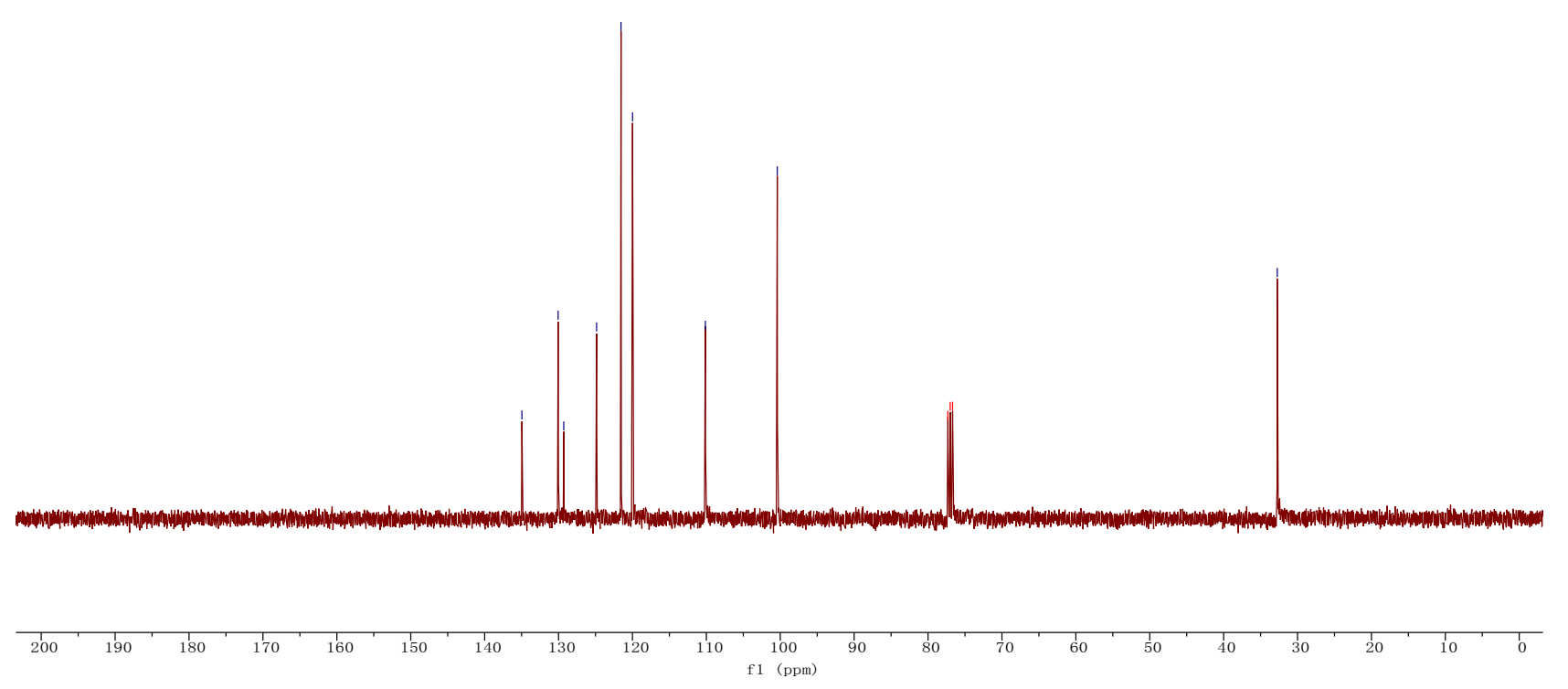


<smiles>Cn1ccc2ccc(F)cc21</smiles>

Compound 2g: $253 \mathrm{mg}, 85 \%$; this is a known compound and its spectroscopic data are consistent with those reported ones. ${ }^{4}{ }^{1} \mathrm{H}$ NMR (400 MHz, $\left.\mathrm{CDCl}_{3}, \mathrm{TMS}\right) \delta 7.50(\mathrm{dd}, J=8.6,5.4 \mathrm{~Hz}, 1 \mathrm{H}), 7.00$ - $6.90(\mathrm{~m}, 2 \mathrm{H}), 6.86(\mathrm{ddd}, J=9.6,8.6,2.3 \mathrm{~Hz}, 1 \mathrm{H}), 6.46-6.41(\mathrm{~m}, 1 \mathrm{H}), 3.67$ (s, 3H); ${ }^{13} \mathrm{C}\left\{{ }^{1} \mathrm{H}\right\}-\mathrm{NMR}\left(100 \mathrm{MHz}, \mathrm{CDCl}_{3}, \mathrm{TMS}\right) \delta_{\mathrm{C}} 159.72(\mathrm{~d}, J=237.2 \mathrm{~Hz}), 136.63(\mathrm{~d}, J=12.0 \mathrm{~Hz})$, $129.16(\mathrm{~d}, J=3.7 \mathrm{~Hz}), 124.8,121.46(\mathrm{~d}, J=10.1 \mathrm{~Hz}), 107.93(\mathrm{~d}, J=24.6 \mathrm{~Hz}), 101.0,95.51(\mathrm{~d}, J=$ 26.1 Hz), 32.8; ${ }^{19} \mathrm{~F}$ NMR (376 MHz, $\left.\mathrm{CDCl}_{3}\right) \delta-121.21$. 


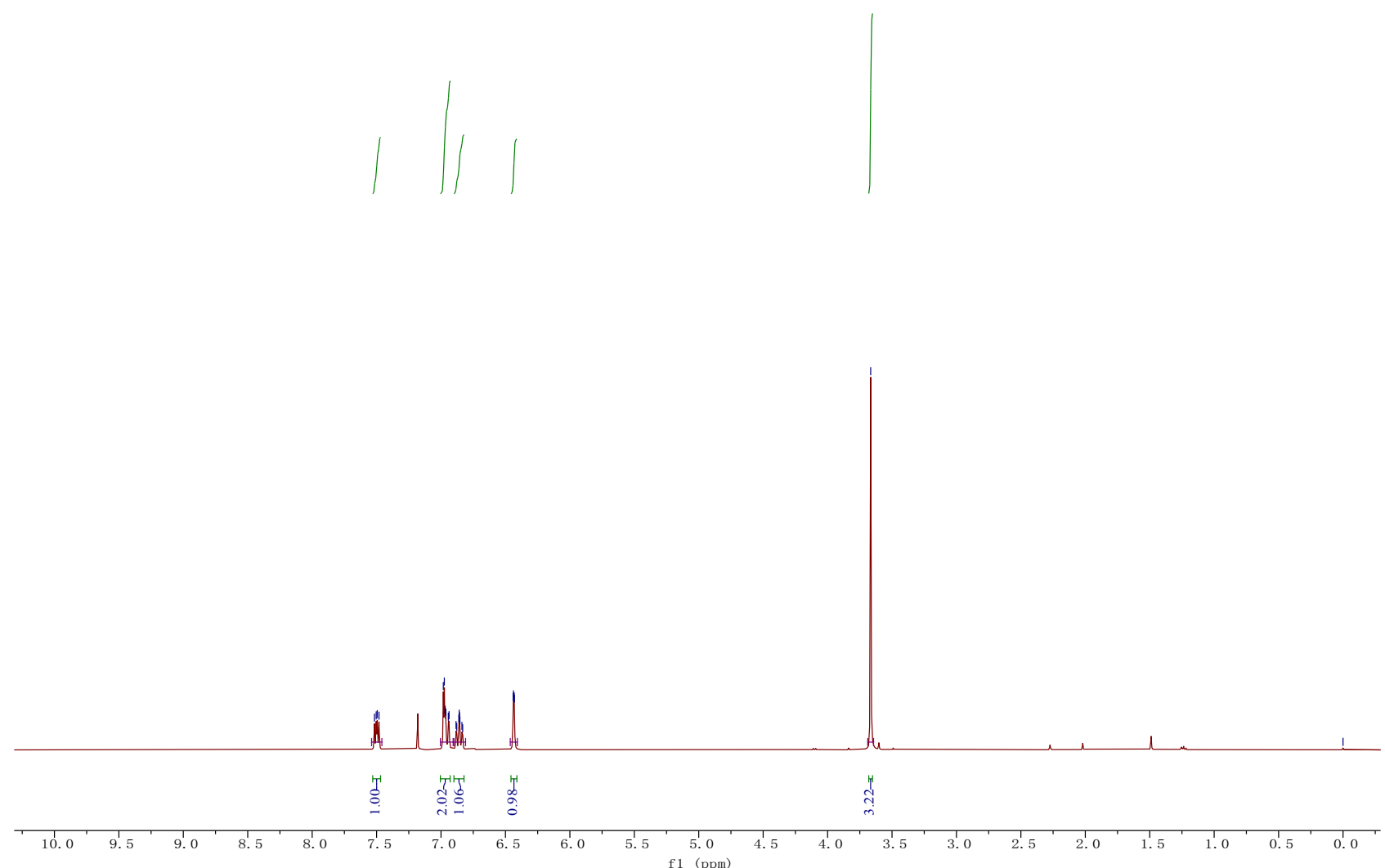

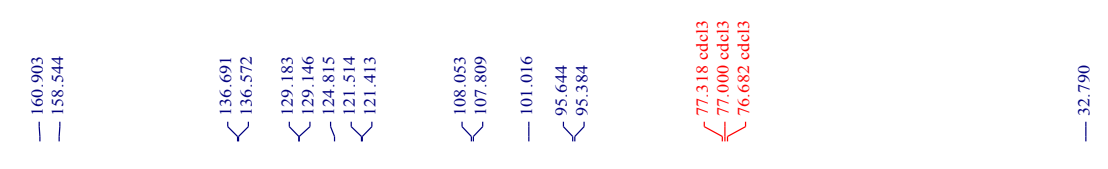

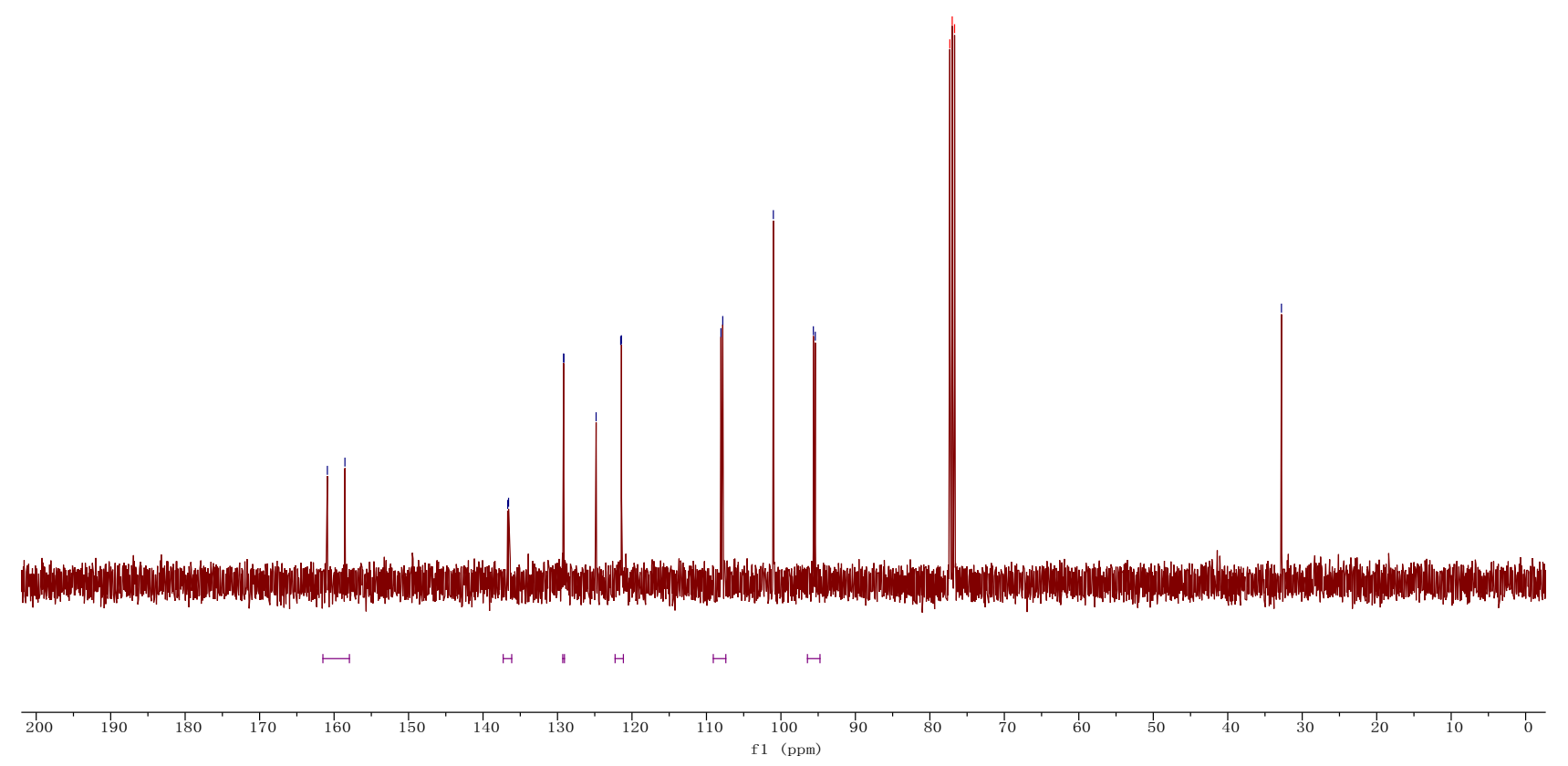




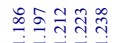

초츠녹

ن

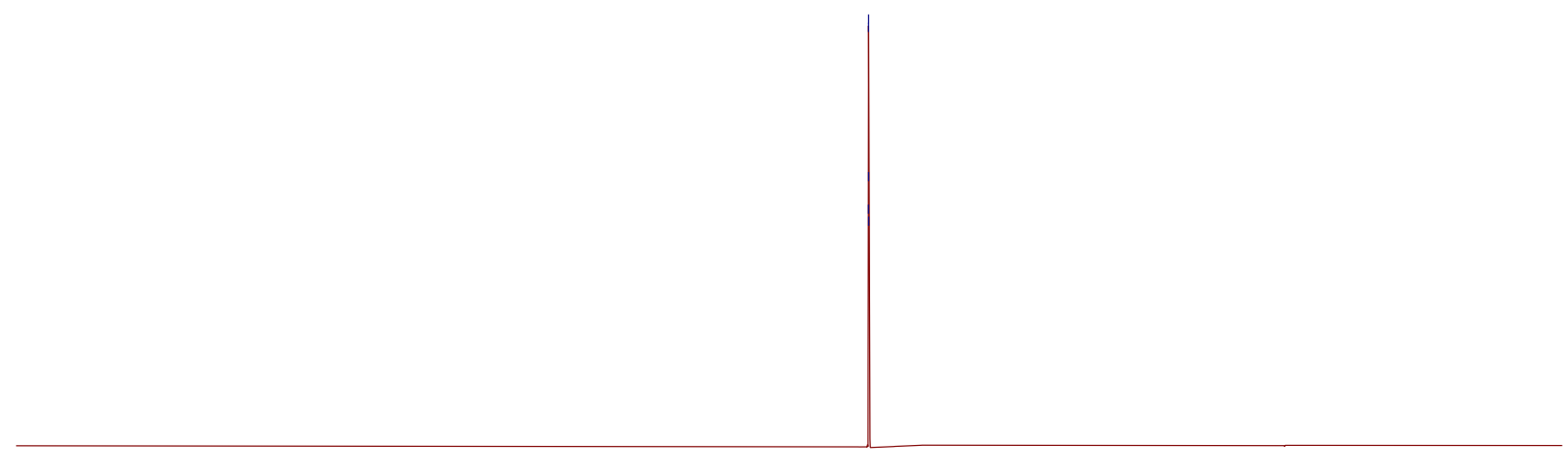

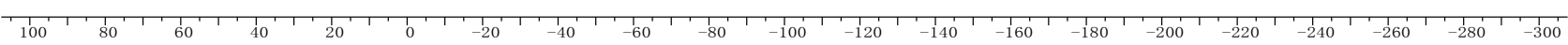


<smiles>Cn1ccc2cc(Br)ccc21</smiles>

Compound 2h: $361 \mathrm{mg}, 86 \%$; this is a known compound and its spectroscopic data are consistent with those reported ones. ${ }^{2}{ }^{1} \mathrm{H}$ NMR $\left(400 \mathrm{MHz} \mathrm{CDCl}_{3}\right.$, TMS) $\delta 7.73(\mathrm{~s}, 1 \mathrm{H}), 7.27$ (d, $J=8.4 \mathrm{~Hz}$, 1H), $7.14(\mathrm{~d}, J=8.7 \mathrm{~Hz}, 1 \mathrm{H}), 7.01(\mathrm{~s}, 1 \mathrm{H}), 6.40(\mathrm{~s}, 1 \mathrm{H}), 3.72(\mathrm{~s}, 3 \mathrm{H}) ;{ }^{13} \mathrm{C}\left\{{ }^{1} \mathrm{H}\right\}-\mathrm{NMR}(100 \mathrm{MHz}$, $\left.\mathrm{CDCl}_{3}, \mathrm{TMS}\right) \delta_{\mathrm{C}} 135.3,130.0,129.9,124.2,123.2,112.6,110.6,100.4,32.9$. 

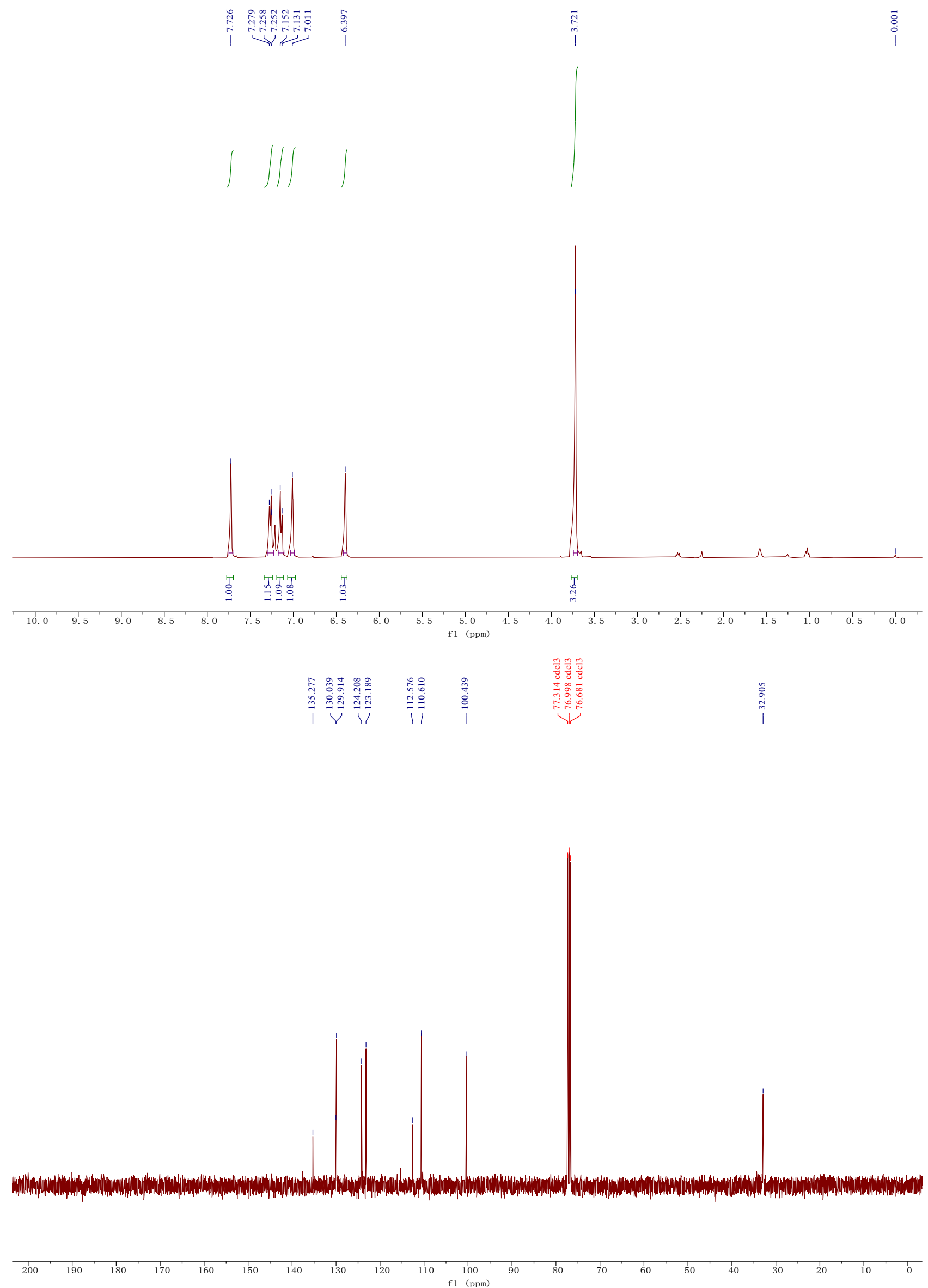


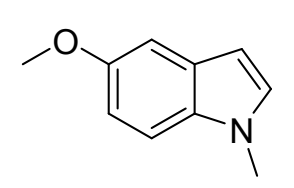

Compound 2i: $267 \mathrm{mg}, 83 \%$; this is a known compound and its spectroscopic data are consistent with those reported ones. ${ }^{2}{ }^{1} \mathrm{H}$ NMR (400 MHz, $\mathrm{CDCl}_{3}$, TMS) $\delta 7.20$ (d, $\left.J=8.8 \mathrm{~Hz}, 1 \mathrm{H}\right), 7.09$ (s, 1H), $7.00(\mathrm{~d}, J=3.1 \mathrm{~Hz}, 1 \mathrm{H}), 6.92-6.85(\mathrm{~m}, 1 \mathrm{H}), 6.39$ (d, $J=3.0 \mathrm{~Hz}, 1 \mathrm{H}), 3.84(\mathrm{~s}, 3 \mathrm{H}), 3.74$ (s, $3 \mathrm{H}) ;{ }^{13} \mathrm{C}\left\{{ }^{1} \mathrm{H}\right\}-\mathrm{NMR}\left(100 \mathrm{MHz}, \mathrm{CDCl}_{3}, \mathrm{TMS}\right) \delta_{\mathrm{C}} 153.9,132.1,129.3,128.7,111.8,109.9,102.4$, 100.3, 55.8, 32.9. 


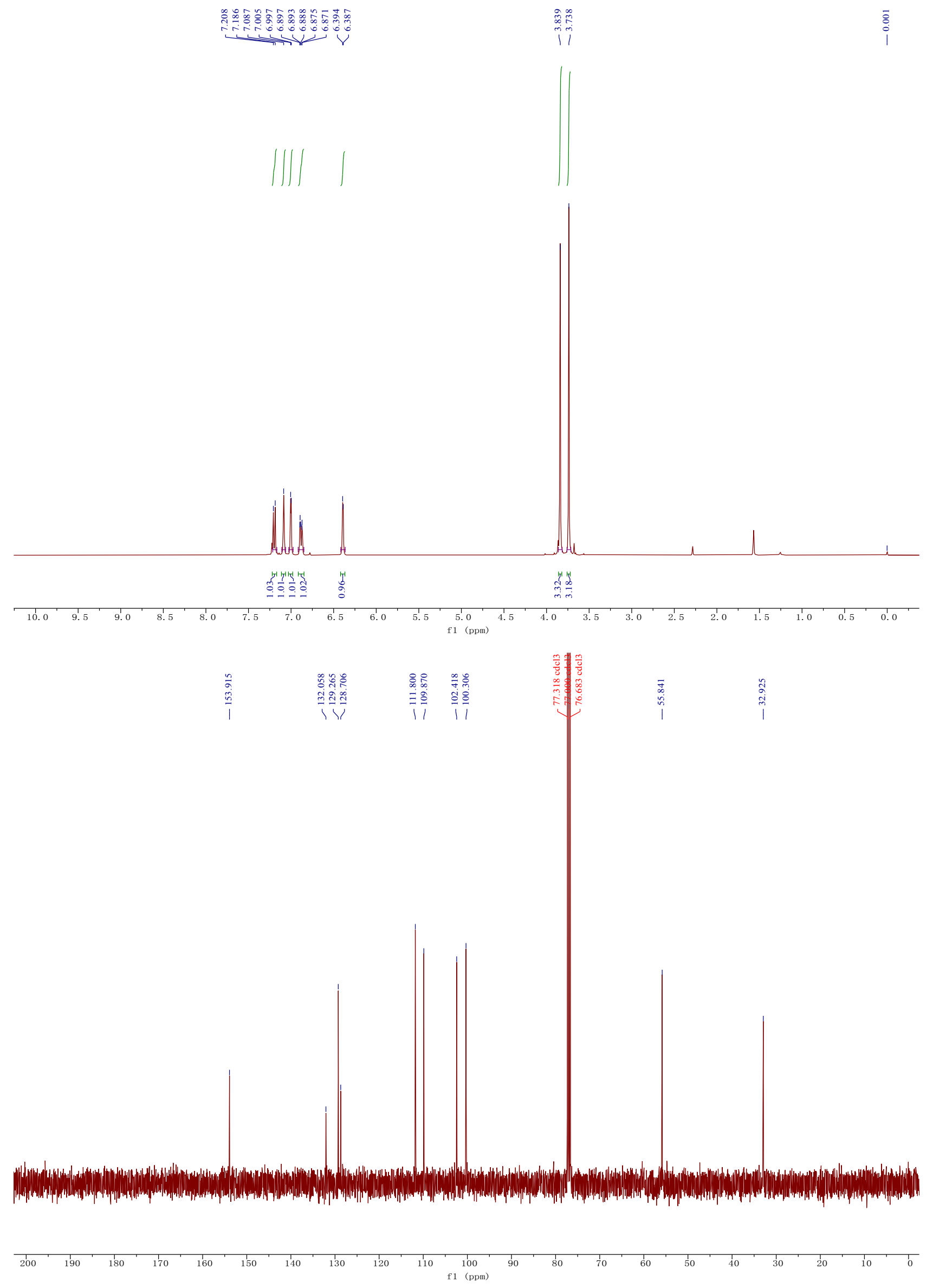




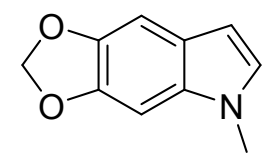

Compound 2j: $280 \mathrm{mg}$, 80\%; this is a known compound and its spectroscopic data are consistent with those reported ones. ${ }^{5}{ }^{1} \mathrm{H}$ NMR (400 MHz, $\mathrm{CDCl}_{3}$, TMS) $\delta 6.99-6.94(\mathrm{~m}, 1 \mathrm{H}), 6.84(\mathrm{dd}, J=$ 3.1, $1.7 \mathrm{~Hz}, 1 \mathrm{H}), 6.72(\mathrm{~s}, 1 \mathrm{H}), 6.31(\mathrm{~d}, J=3.1 \mathrm{~Hz}, 1 \mathrm{H}), 5.86(\mathrm{~d}, J=2.2 \mathrm{~Hz}, 2 \mathrm{H}), 3.60(\mathrm{~s}, 3 \mathrm{H})$; ${ }^{13} \mathrm{C}\left\{{ }^{1} \mathrm{H}\right\}-\mathrm{NMR}\left(100 \mathrm{MHz}, \mathrm{CDCl}_{3}, \mathrm{TMS}\right) \delta_{\mathrm{C}} 144.6,142.6,131.8,127.2,122.0,100.9,100.4,99.2$, 90.1, 32.9. 

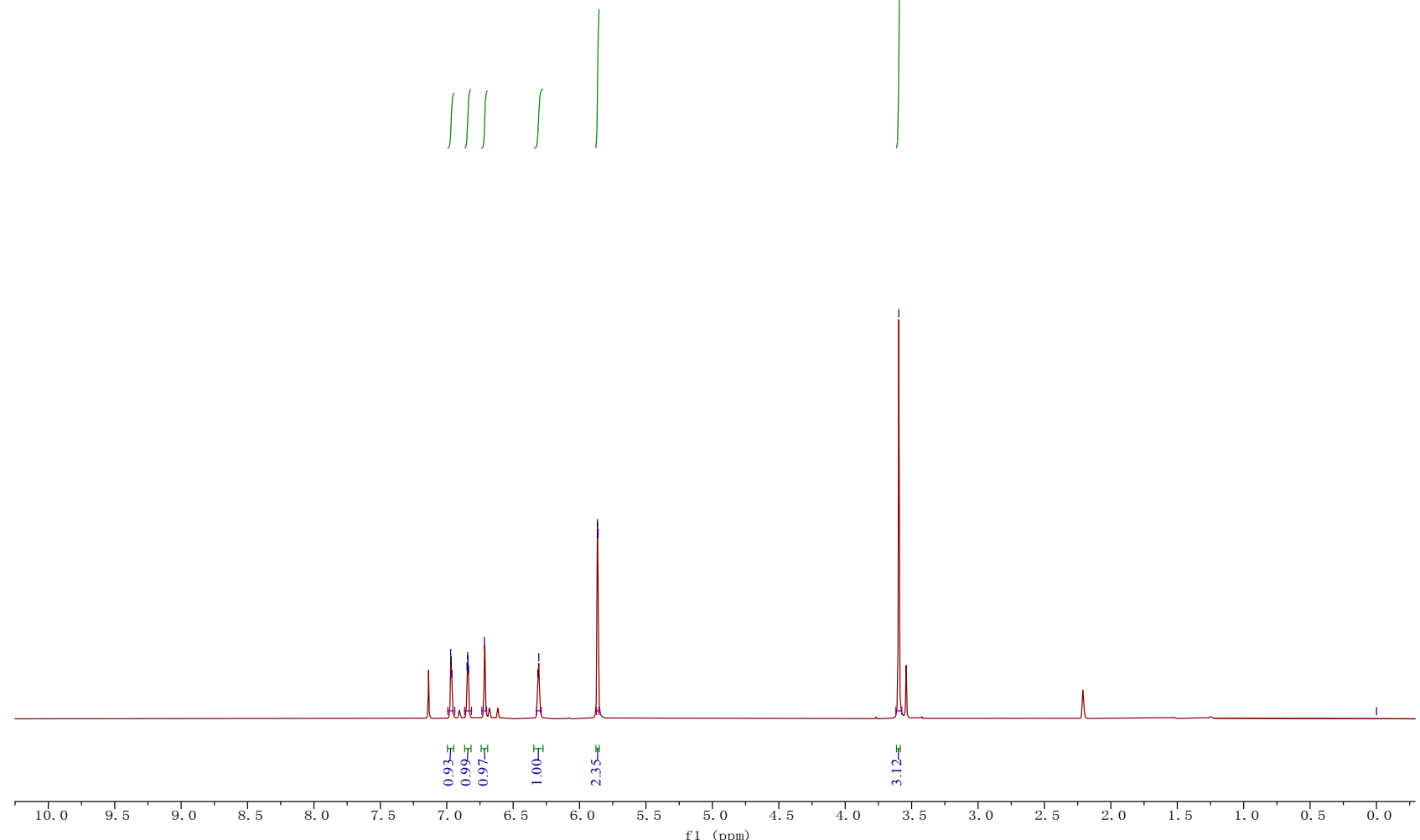

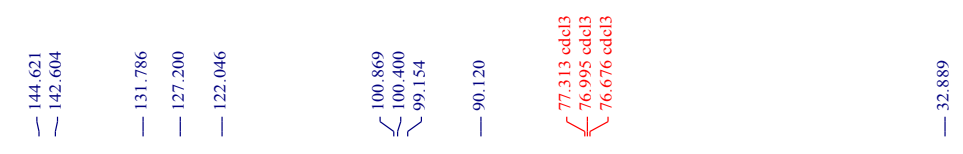

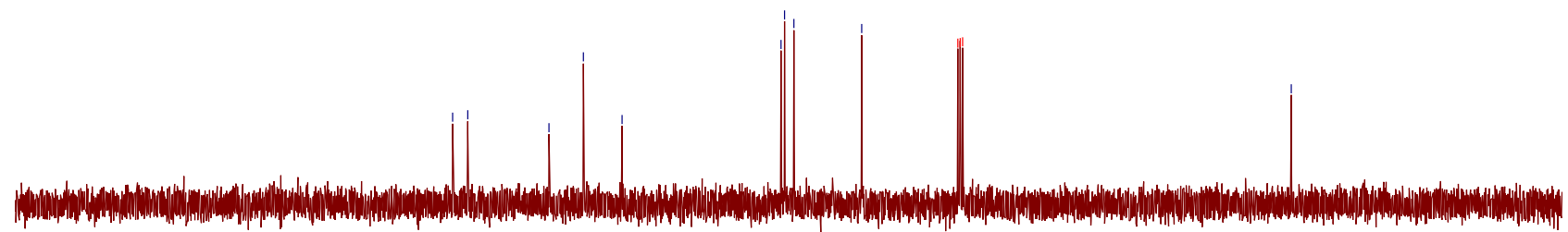

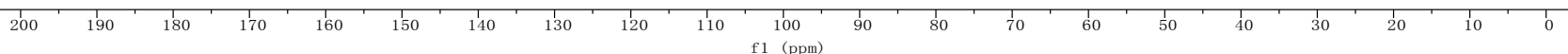




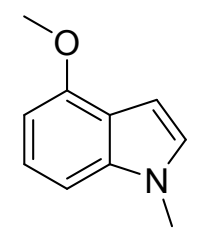

Compound 2k: $264 \mathrm{mg}, 82 \%$; this is a known compound and its spectroscopic data are consistent with those reported ones. ${ }^{6}{ }^{1} \mathrm{H}$ NMR $\left(400 \mathrm{MHz}, \mathrm{CDCl}_{3}, \mathrm{TMS}\right) \delta 7.12(\mathrm{t}, J=8.0 \mathrm{~Hz}, 1 \mathrm{H}), 6.95-6.87$ (m, 2H), $6.57(\mathrm{dd}, J=3.1,0.9 \mathrm{~Hz}, 1 \mathrm{H}), 6.50(\mathrm{~d}, J=7.8 \mathrm{~Hz}, 1 \mathrm{H}), 3.92(\mathrm{~s}, 3 \mathrm{H}), 3.68(\mathrm{~s}, 3 \mathrm{H})$; ${ }^{13} \mathrm{C}\left\{{ }^{1} \mathrm{H}\right\}-\mathrm{NMR}\left(100 \mathrm{MHz}, \mathrm{CDCl}_{3}\right.$, TMS $) \delta_{\mathrm{C}} 153.3,138.1,127.2,122.2,118.9,102.6,99.1,98.1$, 55.2, 32.9 . 

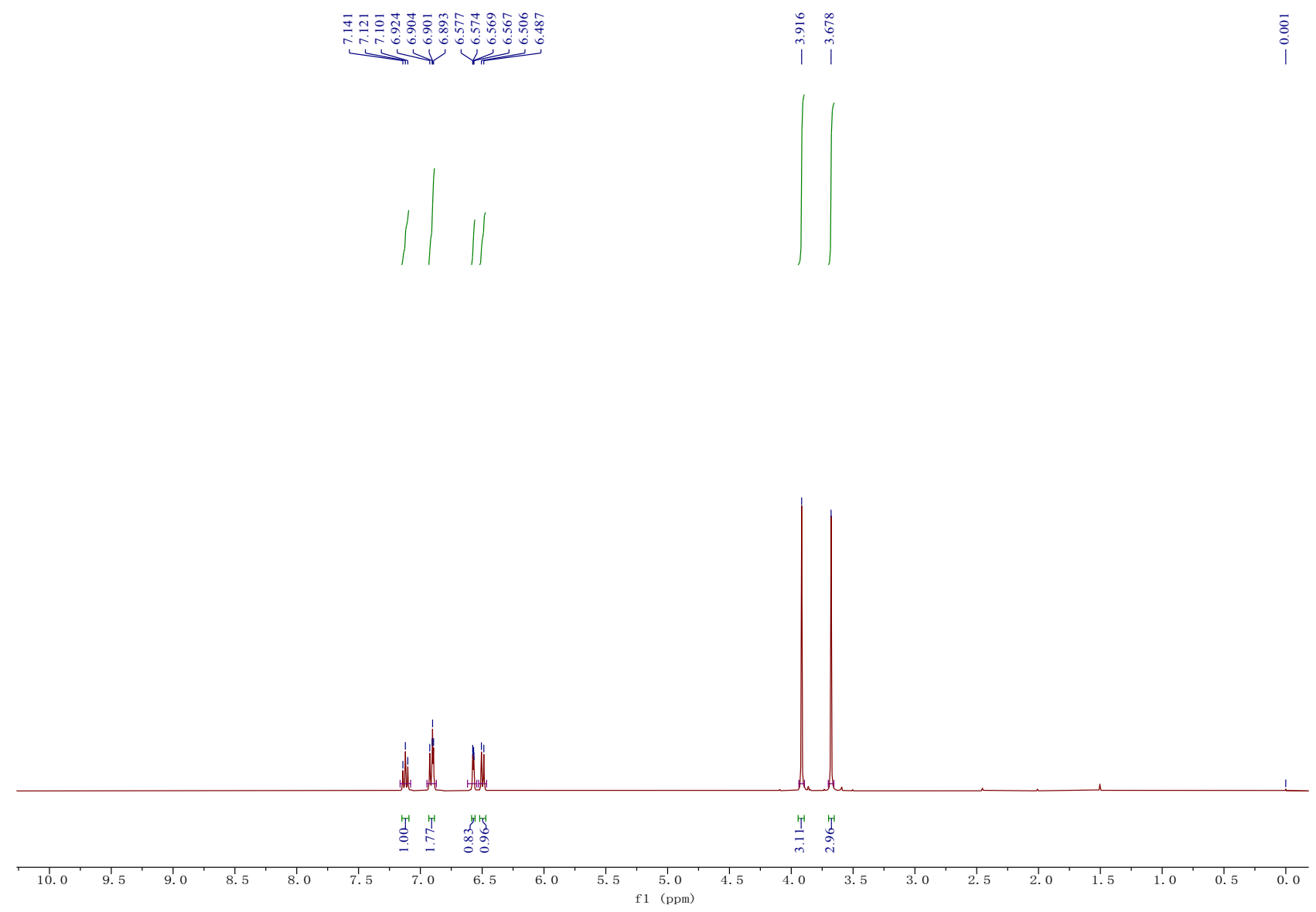

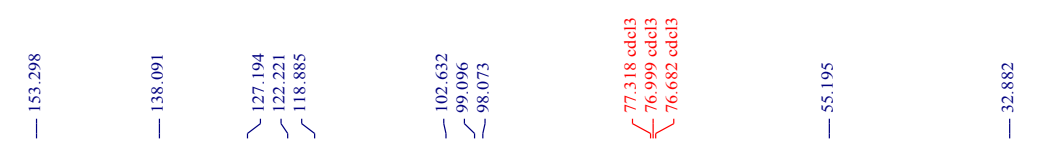

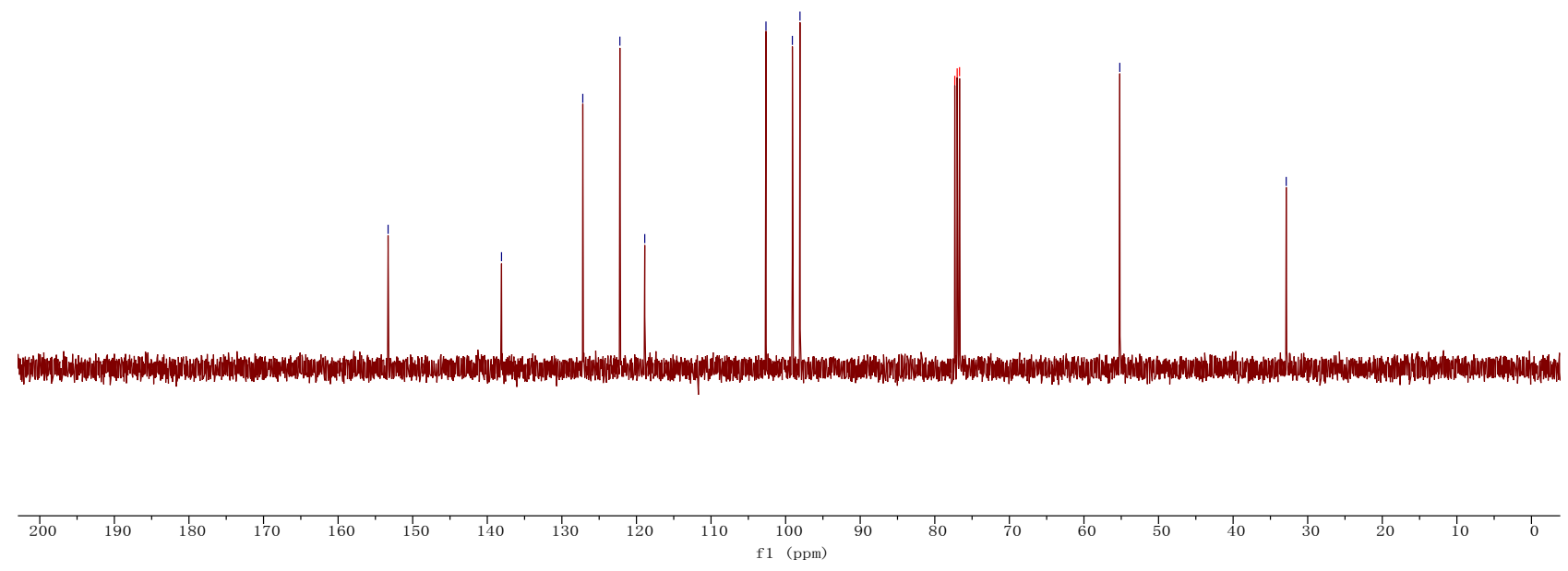




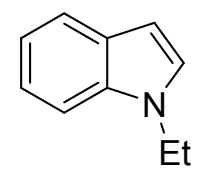

Compound 21: $223 \mathrm{mg}, 77 \%$; this is a known compound and its spectroscopic data are consistent with those reported ones. ${ }^{7}{ }^{1} \mathrm{H} \mathrm{NMR}\left(400 \mathrm{MHz}, \mathrm{CDCl}_{3}, \mathrm{TMS}\right) \delta 7.62(\mathrm{~d}, J=7.9 \mathrm{~Hz}, 1 \mathrm{H}), 7.32(\mathrm{~d}, J$ $=8.2 \mathrm{~Hz}, 1 \mathrm{H}), 7.19(\mathrm{t}, J=7.0 \mathrm{~Hz}, 1 \mathrm{H}), 7.12-7.05(\mathrm{~m}, 2 \mathrm{H}), 6.48(\mathrm{~d}, J=2.7 \mathrm{~Hz}, 1 \mathrm{H}), 4.11(\mathrm{q}, J=$ $7.3 \mathrm{~Hz}, 2 \mathrm{H}), 1.41(\mathrm{t}, J=7.3 \mathrm{~Hz}, 3 \mathrm{H}) ;{ }^{13} \mathrm{C}\left\{{ }^{1} \mathrm{H}\right\}-\mathrm{NMR}\left(100 \mathrm{MHz}, \mathrm{CDCl}_{3}, \mathrm{TMS}\right) \delta_{\mathrm{C}} 135.6,128.6$, $126.9,121.3,120.9,119.1,109.2,100.9,40.9,15.4$. 

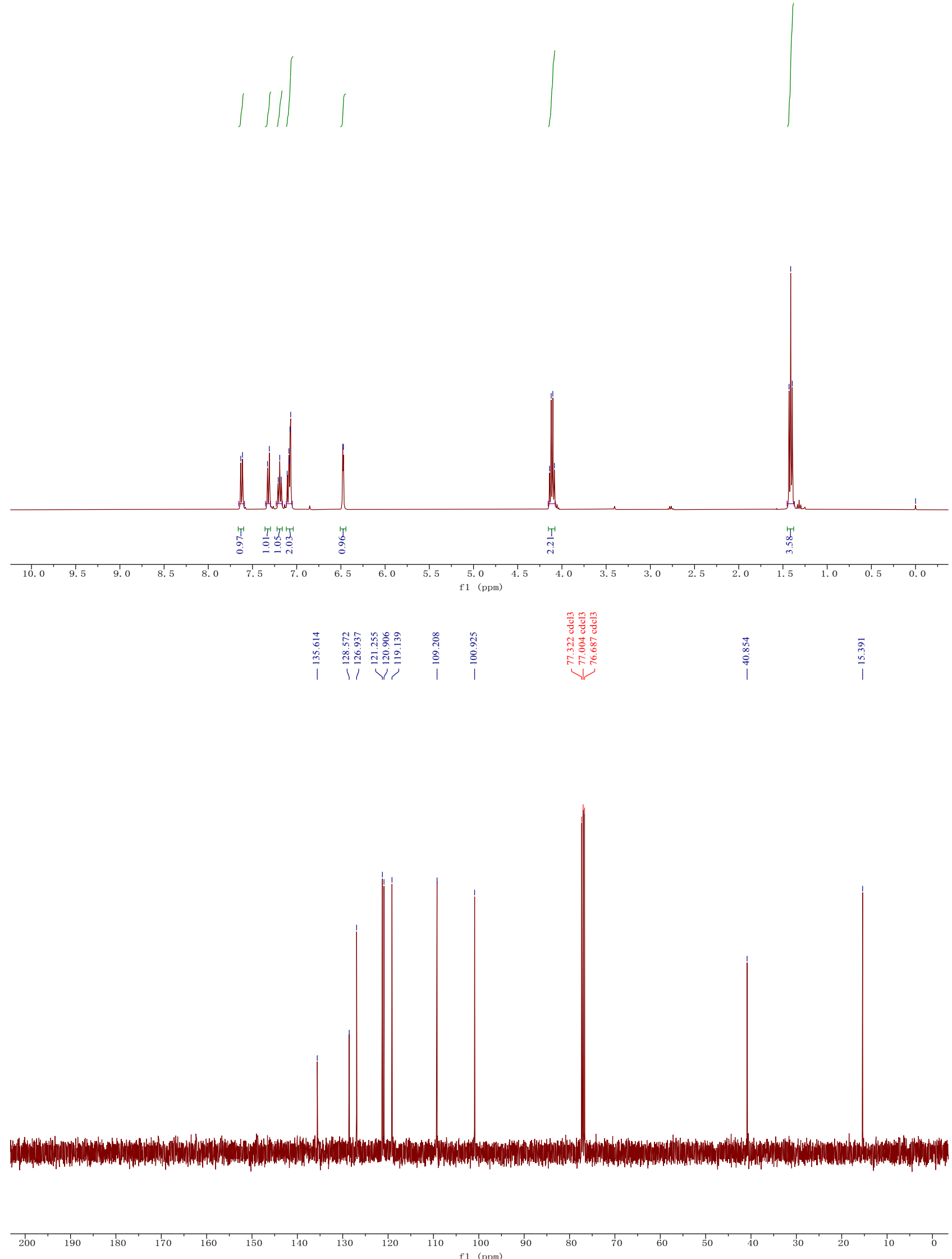


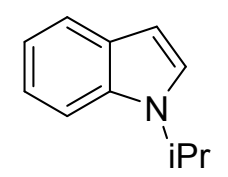

Compound 2n: $193 \mathrm{mg}, 51 \%$; this is a known compound and its spectroscopic data are consistent with those reported ones. ${ }^{8}{ }^{1} \mathrm{H}$ NMR (400 MHz, $\mathrm{CDCl}_{3}$, TMS) $\delta 7.66-7.59(\mathrm{~m}, 1 \mathrm{H}), 7.41-7.34(\mathrm{~m}$, 1H), $7.25-7.15(\mathrm{~m}, 2 \mathrm{H}), 7.09$ (ddd, $J=7.9,7.0,1.0 \mathrm{~Hz}, 1 \mathrm{H}), 6.54-6.48(\mathrm{~m}, 1 \mathrm{H}), 4.67(\mathrm{p}, J=6.7$ $\mathrm{Hz}, 1 \mathrm{H}), 1.52(\mathrm{~d}, J=6.7 \mathrm{~Hz}, 6 \mathrm{H}) ;{ }^{13} \mathrm{C}\left\{{ }^{1} \mathrm{H}\right\}-\mathrm{NMR}\left(100 \mathrm{MHz}, \mathrm{CDCl}_{3}, \mathrm{TMS}\right) \delta_{\mathrm{C}} 135.4,128.5,123.5$, $121.1,120.9,119.2,109.4,101.1,46.9,22.8$. 

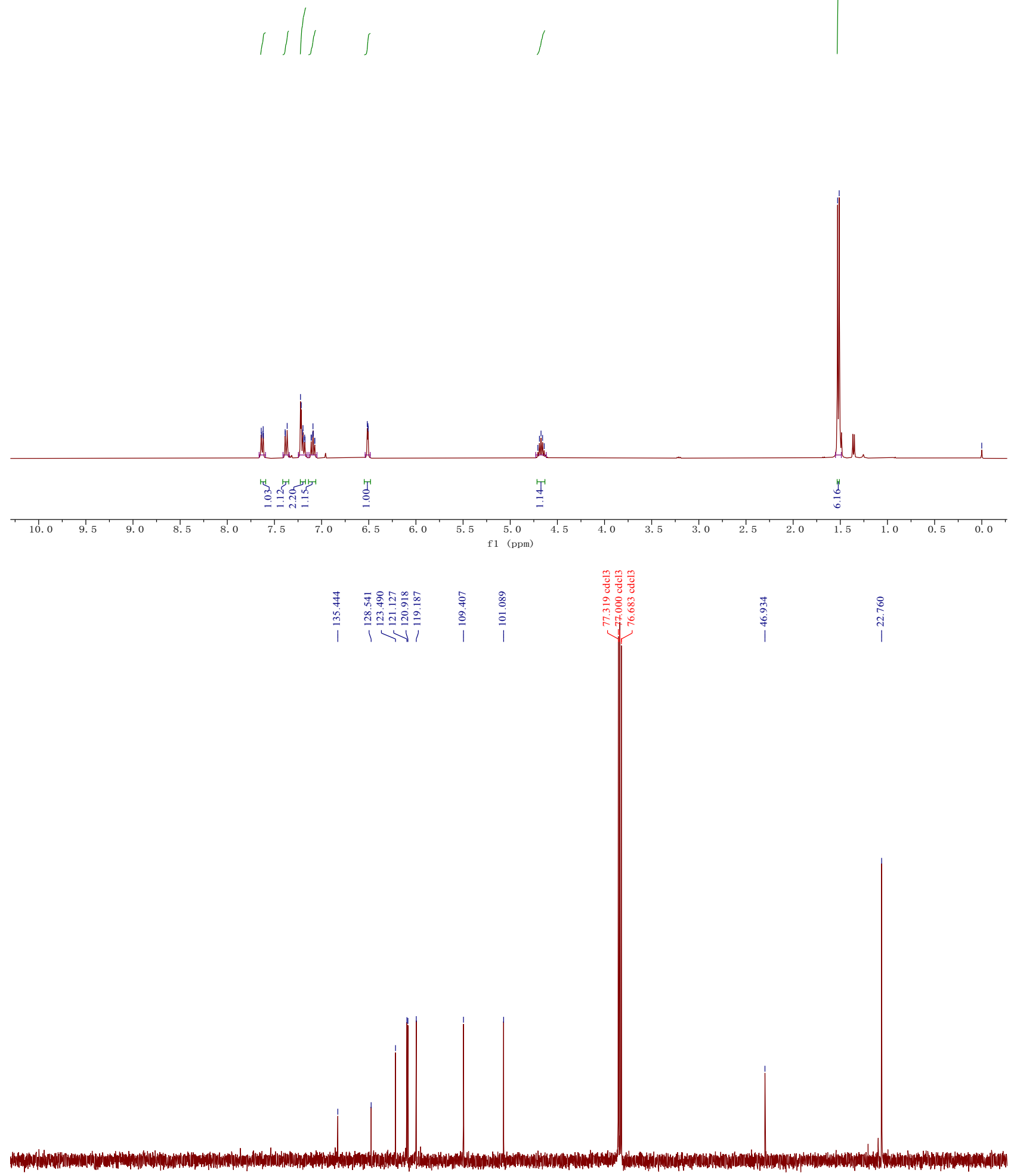

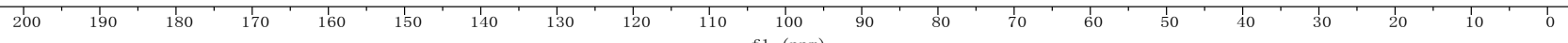




\section{X-ray Data.}

Single crystals suitable for XRD were obtained by vapor diffusion experiment:

Compounds 3a $(50 \mathrm{mg})$ were separately dissolved in $0.5 \mathrm{~mL}$ of dichloromethane and $5 \mathrm{~mL}$ of diethyl ether in three glass vials, which were then placed in sealed glass container. Crystals were obtained in about 4-7 days with the evaporation of the solvent.

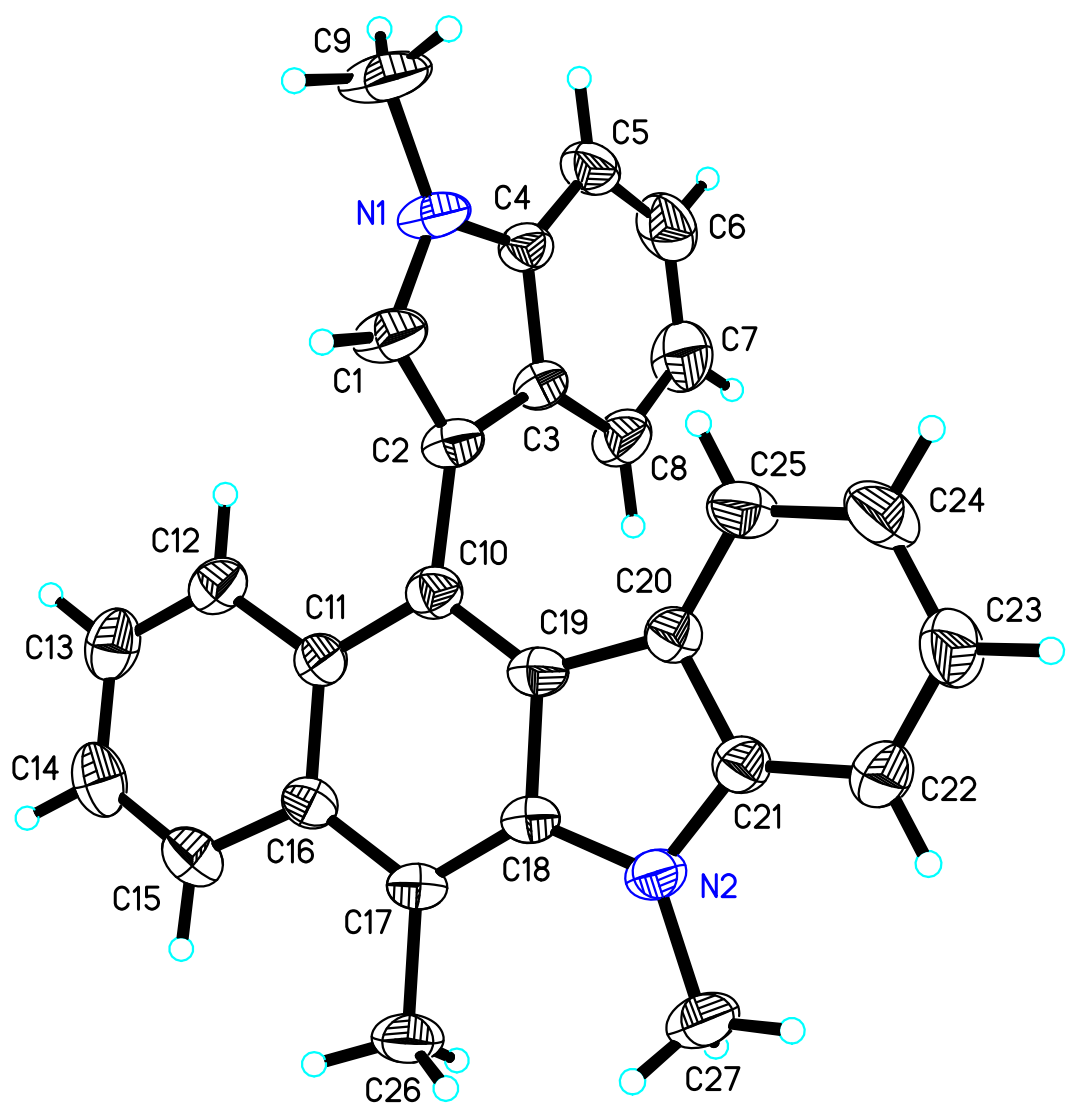

The crystal data of 3a have been deposited in CCDC with number 908539. Empirical Formula: $\mathrm{C}_{27} \mathrm{H}_{22} \mathrm{~N}_{2}$; Formula Weight: 374.47; Crystal Color, Habit: colorless, Crystal Dimensions: $0.276 \mathrm{x}$ 0.212 x $0.145 \mathrm{~mm}^{3}$; Crystal System: Monoclinic; Lattice Parameters: $\mathrm{a}=14.0271(12) \AA, \mathrm{b}=$ 7.8971(7) $\AA, \mathrm{c}=18.8147(16) \AA, \alpha=90^{\circ}, \beta=104.462(2)^{\circ}, \gamma=90^{\circ}, \mathrm{V}=2018.1(3) \AA^{3}$; Space group: $\mathrm{P} 2(1) / \mathrm{c} ; \mathrm{Z}=4 ; \mathrm{D}_{\text {calc }}=1.232 \mathrm{~g} / \mathrm{cm}^{3} ; \mathrm{F}_{000}=792 ;$ Final R induces $[\mathrm{I}>2 \operatorname{sigma}(\mathrm{I})]: \mathrm{R} 1=0.0553 ; \mathrm{wR} 2=$ 0.1567 . The thermal ellipsoids are set at a $30 \%$ probability level. 
Single crystals suitable for XRD were obtained by vapor diffusion experiment:

Compounds $\mathbf{3 j}$ (50 mg) were separately dissolved in $0.5 \mathrm{~mL}$ of dichloromethane and $5 \mathrm{~mL}$ of ethanol in three glass vials, which were then placed in sealed glass container. Crystals were obtained in about 10-20 days with the evaporation of the solvent.

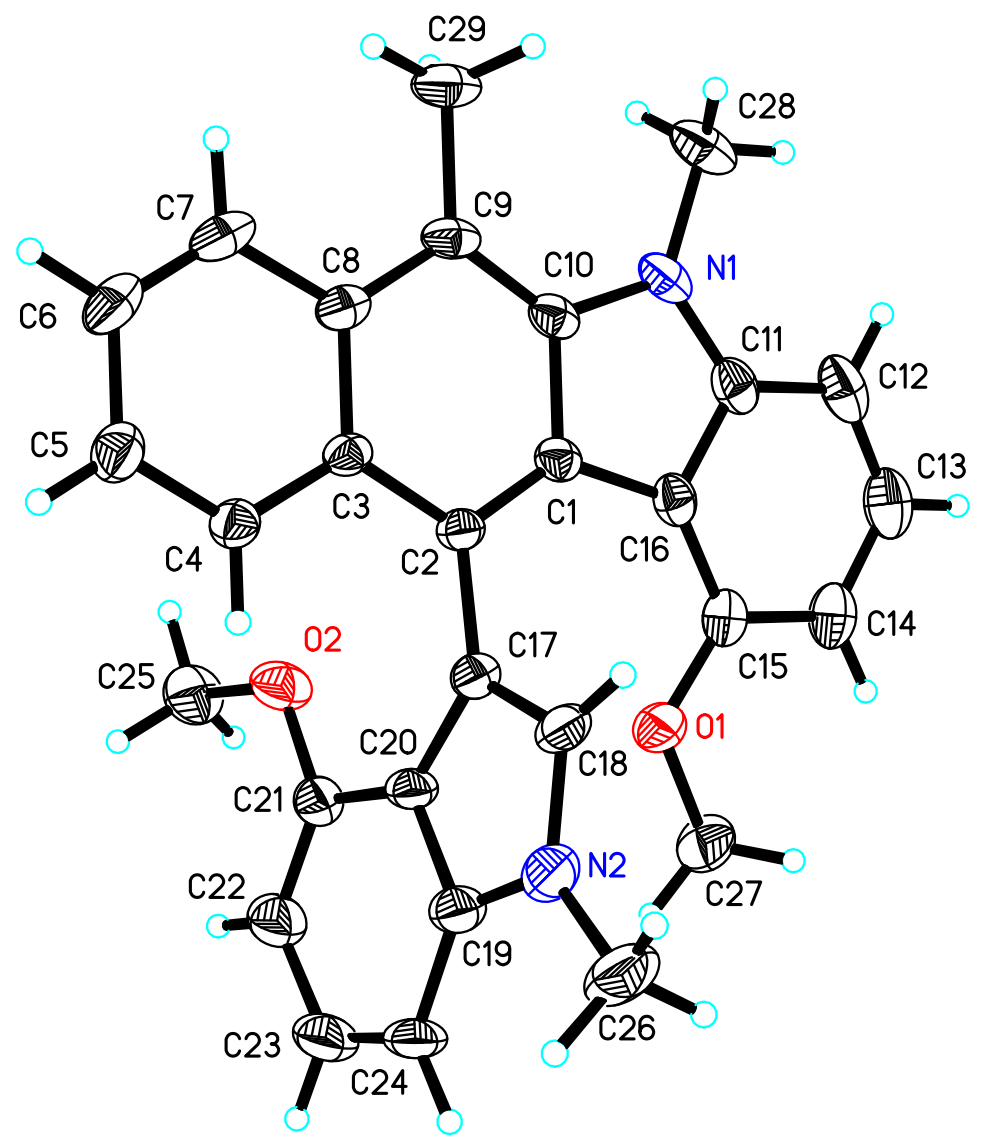

The crystal data of 3k have been deposited in CCDC with number 2082031. Empirical Formula: $\mathrm{C}_{29} \mathrm{H}_{26} \mathrm{~N}_{2} \mathrm{O}_{2}$; Formula Weight: 434.52; Crystal Color, Habit: colorless, Crystal Dimensions: $0.200 \mathrm{x}$ 0.150 × $0.120 \mathrm{~mm}$; Crystal System: Monoclinic; Lattice Parameters: $\mathrm{a}=12.9570(5) \AA, \mathrm{b}=$ 7.3163(3) $\AA, c=23.7679(8) \AA, \alpha=90^{\circ}, \beta=97.2720(10)^{\circ}, \gamma=90^{\circ}, V=2235.01(15) \AA^{3}$; Space group: P 21/c; Z $=4 ; D_{\text {calc }}=1.291 \mathrm{~g} / \mathrm{cm} 3 ; \mathrm{F}_{000}=920$; Final R induces $[\mathrm{I}>2 \operatorname{sigma}(\mathrm{I})]: \mathrm{R} 1=0.0677 ; \mathrm{wR} 2=$ 0.1843 . The thermal ellipsoids are set at a $30 \%$ probability level. 


\section{References.}

1) Acqua, M. D.; Facoetti, D.; Abbiati, G.; Rossi E. Synthesis 2010, 14, 2367.

2) Kim, J.; Kim, H.; and Chang, S. Org. Lett. 2012, 14, 3924.

3) Ding, T.-H.; Qu, J.-P.; Kang Y.-B. Org. Lett. 2020, 22, 3084.

4) Su, Y.-M.; Yu, H.; Yin, F.; Xu Y.-M.; Li, Y.; Zheng, X.; Wang X.-S. Org. Lett. 2014, 16, 2958.

5) Santoso, M.; Somphol, K.; Kumar, N.; Black, D. Tetrahedron 2009, 65, 5977.

6) Greulich, T. W.; Daniliuc, C. G.; Studer A. Org. Lett. 2015, 17 , 254.

7) Xu, X.-H.; Liu, G.-K.; Azuma, A.; Tokunaga, E.; Shibata, N. Org. Lett. 2011, 13, 4854.

8) Turnu, F.; Luridiana, A. Cocco, A.; Porcu, S. Frongia, A.; Sarais, G.; Secci, F. Org. Lett. 2019, 21,7329 . 